Prediction of Structures and Atomization Energies of Small Silver Clusters, (Ag), $\mathbf{n}$ $<\mathbf{1 0 0}$

Mingyang Chen, Jason E. Dyer, Keijing Li, and David A. Dixon ${ }^{* \dagger}$

Department of Chemistry, The University of Alabama, Shelby Hall, Tuscaloosa, AL, 35487-0336, USA

\title{
Ref. 36
}

Gaussian 09, Revision B.1, Frisch, M. J.; Trucks, G. W.; Schlegel, H. B.; Scuseria, G. E.; Robb, M. A.; Cheeseman, J. R.; Scalmani, G.; Barone, V.; Mennucci, B.; Petersson, G. A.; Nakatsuji, H.; Caricato, M.; Li, X.; Hratchian, H. P.; Izmaylov, A. F.; Bloino, J.; Zheng, G.; Sonnenberg, J. L.; Hada, M.; Ehara, M.; Toyota, K.; Fukuda, R.; Hasegawa, J.; Ishida, M.; Nakajima, T.; Honda, Y.; Kitao, O.; Nakai, H.; Vreven, T.; Montgomery, Jr., J. A.; Peralta, J. E.; Ogliaro, F.; Bearpark, M.; Heyd, J. J.; Brothers, E.; Kudin, K. N.; Staroverov, V. N.; Kobayashi, R.; Normand, J.; Raghavachari, K.; Rendell, A.; Burant, J. C.; Iyengar, S. S.; Tomasi, J.; Cossi, M.; Rega, N.; Millam, N. J.; Klene, M.; Knox, J. E.; Cross, J. B.; Bakken, V.; Adamo, C.; Jaramillo, J.; Gomperts, R.; Stratmann, R. E.; Yazyev, O.; Austin, A. J.; Cammi, R.; Pomelli, C.; Ochterski, J. W.; Martin, R. L.; Morokuma, K.; Zakrzewski, V. G.; Voth, G. A.; Salvador, P.; Dannenberg, J. J.; Dapprich, S.; Daniels, A. D.; Farkas, Ö.; Foresman, J. B.; Ortiz, J. V.; Cioslowski, J.; Fox, D. J. Gaussian, Inc., Wallingford CT, 2009.

\section{Ref. 44(b)}

MOLPRO, version 2010.1, a package of ab initio programs, Werner, H.-J.; Knowles, P. J.; Manby, F. R.; Schütz, M.; Celani, P.; Knizia, G.; Korona, T.; Lindh, R.; Mitrushenkov, A.; Rauhut, G.; Adler, T. B.; Amos, R. D.; Bernhardsson, A.; Berning, A.; Cooper, D. L.; Deegan, M. J. O.; Dobbyn, A. J.; Eckert, F.; Goll, E.; Hampel, C.; Hesselmann, A.; Hetzer, G.; Hrenar, T.; Jansen, G.; Köppl, C.; Liu, Y.; Lloyd, A. W.; Mata, R. A.; May, A. J.; McNicholas, S. J.; Meyer, W.; Mura, M. E.; Nicklass, A.; Palmieri, P.; Pflüger, K.; Pitzer, R.; Reiher, M.; Shiozaki, T.; Stoll, H.; Stone, A. J.; Tarroni, R.; Thorsteinsson, T.; Wang, M.; Wolf, A., see http://www.molpro.net

\footnotetext{
†Email: dadixon@bama.ua.edu
} 
Table S1. Average bond lengths ( $R$ ) and coordination numbers $(\mathrm{CN})$ for $\operatorname{Ag}_{\mathrm{n}}, \mathbf{n}=\mathbf{2 1 -}$ 99 using TG-HGA(EAM).

\begin{tabular}{|c|c|c|}
\hline $\mathrm{n}$ & average $\mathrm{R}$ & average $\mathrm{CN}$ \\
\hline 21 & 2.844 & 7.24 \\
\hline 22 & 2.847 & 7.27 \\
\hline 23 & 2.852 & 7.57 \\
\hline 24 & 2.861 & 7.50 \\
\hline 25 & 2.854 & 7.44 \\
\hline 26 & 2.854 & 7.62 \\
\hline 27 & 2.851 & 7.41 \\
\hline 28 & 2.859 & 7.71 \\
\hline 29 & 2.867 & 7.79 \\
\hline 30 & 2.848 & 7.47 \\
\hline 31 & 2.867 & 7.74 \\
\hline 32 & 2.835 & 7.50 \\
\hline 33 & 2.858 & 7.76 \\
\hline 34 & 2.840 & 7.47 \\
\hline 35 & 2.856 & 7.71 \\
\hline 36 & 2.838 & 7.67 \\
\hline 37 & 2.863 & 7.95 \\
\hline 38 & 2.830 & 7.58 \\
\hline 39 & 2.842 & 7.85 \\
\hline 40 & 2.853 & 7.90 \\
\hline 41 & 2.843 & 7.85 \\
\hline 42 & 2.843 & 7.90 \\
\hline 43 & 2.844 & 7.95 \\
\hline 44 & 2.844 & 7.95 \\
\hline 45 & 2.845 & 8.00 \\
\hline 46 & 2.847 & 8.09 \\
\hline 47 & 2.847 & 8.09 \\
\hline 48 & 2.848 & 8.13 \\
\hline
\end{tabular}




\begin{tabular}{|c|c|c|}
\hline 49 & 2.849 & 8.20 \\
\hline 50 & 2.850 & 8.20 \\
\hline 51 & 2.850 & 8.24 \\
\hline 52 & 2.851 & 8.31 \\
\hline 53 & 2.853 & 8.38 \\
\hline 54 & 2.854 & 8.44 \\
\hline 55 & 2.855 & 8.51 \\
\hline 56 & 2.855 & 8.46 \\
\hline 57 & 2.855 & 8.46 \\
\hline 58 & 2.855 & 8.48 \\
\hline 59 & 2.860 & 8.51 \\
\hline 60 & 2.856 & 8.50 \\
\hline 61 & 2.856 & 8.52 \\
\hline 62 & 2.8559 & 8.52 \\
\hline 63 & 2.8566 & 8.54 \\
\hline 64 & 2.8572 & 8.56 \\
\hline 65 & 2.8571 & 8.55 \\
\hline 66 & 2.8576 & 8.58 \\
\hline 67 & 2.8583 & 8.60 \\
\hline 68 & 2.8585 & 8.59 \\
\hline 69 & 2.8453 & 8.35 \\
\hline 70 & 2.8601 & 8.69 \\
\hline 71 & 2.8653 & 8.73 \\
\hline 72 & 2.8466 & 8.42 \\
\hline 73 & 2.847 & 8.44 \\
\hline 74 & 2.8473 & 8.46 \\
\hline 75 & 2.8484 & 8.51 \\
\hline 76 & 2.8482 & 8.50 \\
\hline 77 & 2.8485 & 8.52 \\
\hline 78 & 2.8486 & 8.51 \\
\hline 79 & 2.8481 & 8.48 \\
\hline
\end{tabular}




\begin{tabular}{|l|l|l|}
\hline 80 & 2.8565 & 8.70 \\
\hline 81 & 2.8567 & 8.72 \\
\hline 82 & 2.8569 & 8.73 \\
\hline 83 & 2.857 & 8.75 \\
\hline 84 & 2.857 & 8.74 \\
\hline 85 & 2.8495 & 8.56 \\
\hline 86 & 2.8488 & 8.56 \\
\hline 87 & 2.8578 & 8.78 \\
\hline 89 & 2.8584 & 8.83 \\
\hline 90 & 2.8585 & 8.84 \\
\hline 91 & 2.8587 & 8.86 \\
\hline 92 & 2.8588 & 8.87 \\
\hline 93 & 2.8589 & 8.86 \\
\hline 94 & 2.8591 & 8.87 \\
\hline 95 & 2.8591 & 8.88 \\
\hline 96 & 2.8511 & 8.71 \\
\hline 97 & 2.8512 & 8.72 \\
\hline 98 & 2.8596 & 8.92 \\
\hline 99 & 2.8601 & 8.95 \\
\hline & & \\
\hline
\end{tabular}


Table S2. Vibrational Frequencies $\left(>60 \mathrm{~cm}^{-1}\right.$ for $\mathrm{n}=1-10,>100 \mathrm{~cm}^{-1}$ for $\left.\mathrm{n}=11-20\right)$ for $\operatorname{Ag}_{\mathbf{n}}(\mathbf{n}=\mathbf{2 - 8})$.

\begin{tabular}{|c|c|c|c|c|c|c|c|c|c|}
\hline $\mathbf{A g}_{2}$ & $\mathbf{A g}_{3}$ & $\mathbf{A g}_{4}$ & $\mathbf{A g}_{5}$ & $\mathbf{A g}_{6}$ & $\mathbf{A g}_{7}$ & $\mathbf{A g}_{8}$ & $\mathbf{A g}_{9}$ & $\mathbf{A g}_{10}$ & $\mathbf{A g}_{11}$ \\
\hline$D_{\infty h}$ & $\mathrm{C}_{2 \mathrm{v}}$ & $\mathrm{D}_{2 \mathrm{~h}}$ & $\mathrm{C}_{2 \mathrm{v}}$ & $\mathrm{D}_{3 \mathrm{~h}}$ & $\mathrm{D}_{5 \mathrm{~h}}$ & $\mathrm{~T}_{\mathrm{d}}$ & $\mathrm{C}_{2 \mathrm{v}}$ & $\mathrm{C}_{\mathrm{s}}$ & $\mathrm{C}_{2 \mathrm{v}}$ \\
\hline \multirow[t]{16}{*}{179} & 106 & 77 & 69 & 68 & $71 \mathrm{e}_{1}^{\prime}$ & $62 \mathrm{t}_{2}$ & 60 & 63 & 104 \\
\hline & 175 & 78 & 74 & 83 & $73 \mathrm{a}_{1}^{\prime}$ & $81 \mathrm{t}_{2}$ & 65 & 63 & 110 \\
\hline & & 101 & 93 & 115 & $83 \mathrm{e}_{2}^{\prime}$ & $89 t_{1}$ & 71 & 75 & 111 \\
\hline & & 151 & 111 & 129 & $86 \mathrm{a}_{2} "$ & $95 \mathrm{a}_{1}$ & 73 & 81 & 119 \\
\hline & & 177 & 135 & 134 & $96 \mathrm{e}_{1}$ & $112 \mathrm{e}$ & 73 & 83 & 126 \\
\hline & & & 145 & 171 & $109 \mathrm{e}_{2}^{\prime}$ & $148 \mathrm{t}_{2}$ & 78 & 91 & 132 \\
\hline & & & 173 & & $143 \mathrm{e}_{1}^{\prime}$ & $148 \mathrm{a}_{1}$ & 80 & 103 & 134 \\
\hline & & & & & $153 \mathrm{a}_{1}^{\prime}$ & & 82 & 107 & 139 \\
\hline & & & & & & & 90 & 121 & 145 \\
\hline & & & & & & & 111 & 126 & 156 \\
\hline & & & & & & & 115 & 136 & \\
\hline & & & & & & & 124 & 151 & \\
\hline & & & & & & & 126 & 152 & \\
\hline & & & & & & & 140 & 166 & \\
\hline & & & & & & & 146 & 181 & \\
\hline & & & & & & & 160 & & \\
\hline $\mathbf{A g}_{12}$ & $\mathbf{A g}_{13}$ & $\mathbf{A g}_{14}$ & $\mathbf{A g}_{15}$ & $\mathbf{A g}_{16}$ & $\mathbf{A g}_{17}$ & $\mathbf{A g}_{18}$ & $\mathbf{A g}_{19}$ & $\mathbf{A g}_{20}$ & \\
\hline $\mathrm{C}_{\mathrm{s}}$ & $\mathrm{C}_{2}$ & $\mathrm{C}_{3 \mathrm{v}}$ & $\overline{C_{s}}$ & $\mathrm{C}_{1}$ & $\mathrm{C}_{2}$ & $\mathrm{C}_{\mathrm{s}}$ & $\mathrm{C}_{\mathrm{s}}$ & $\mathrm{T}_{\mathrm{d}}$ & \\
\hline 105 & 100 & $60 \mathrm{e}$ & 101 & 106 & 104 & 100 & 106 & $105 \mathrm{a}_{1}$ & \\
\hline 119 & 102 & $65 \mathrm{a}_{1}$ & 110 & 111 & 108 & 105 & 107 & $116 \mathrm{e}$ & \\
\hline 129 & 108 & $68 \mathrm{e}$ & 111 & 115 & 110 & 109 & 113 & $118 \mathrm{t}_{1}$ & \\
\hline 137 & 117 & $70 a_{1}$ & 115 & 116 & 113 & 111 & 117 & $123 \mathrm{t}_{2}$ & \\
\hline 142 & 121 & $71 \mathrm{a}_{2}$ & 126 & 118 & 117 & 114 & 119 & $137 \mathrm{t}_{2}$ & \\
\hline 147 & 126 & $74 \mathrm{e}$ & 137 & 123 & 117 & 118 & 121 & $160 \mathrm{e}$ & \\
\hline \multirow[t]{3}{*}{164} & 132 & $80 \mathrm{e}$ & 140 & 130 & 122 & 120 & 122 & $167 \mathrm{t}_{2}$ & \\
\hline & 136 & $84 a_{1}$ & 148 & 134 & 130 & 123 & 133 & $172 a_{1}$ & \\
\hline & 144 & $89 \mathrm{a}_{2}$ & 157 & 137 & 133 & 128 & 139 & & \\
\hline
\end{tabular}




\begin{tabular}{|l|c|c|c|c|c|c|c|c|c|}
\hline & 144 & $98 \mathrm{e}$ & 162 & 139 & 135 & 130 & 144 & & \\
\hline & 154 & $102 \mathrm{e}$ & 166 & 141 & 136 & 133 & 152 & & \\
\hline & 156 & $107 \mathrm{a} 1$ & & 155 & 138 & 136 & 154 & & \\
\hline & & $118 \mathrm{a} 1$ & & 165 & 143 & 138 & 158 & & \\
\hline & & $130 \mathrm{e}$ & & & 153 & 140 & 160 & & \\
\hline & & $132 \mathrm{a} 1$ & & & 156 & 146 & 166 & & \\
\hline & & $145 \mathrm{e}$ & & & & 148 & & & \\
\hline & & & & & & 152 & & & \\
\hline & & & & & & 162 & & & \\
\hline
\end{tabular}


Table S3. Reaction Energies for $\operatorname{Ag}_{n+1} \rightarrow A_{n}+A g$ at the DFT/LANL2DZ levels in kcal/mol.

\begin{tabular}{|c|c|c|}
\hline $\mathrm{n}$ & PW91 & $\omega B 97 X D$ \\
\hline 2 & 41.1 & 37.4 \\
\hline 3 & 19.8 & 16.5 \\
\hline 4 & 45.6 & 43.3 \\
\hline 5 & 38.7 & 34.3 \\
\hline 6 & 52.5 & 52.1 \\
\hline 7 & 37.1 & 30.9 \\
\hline 8 & 51.6 & 52.4 \\
\hline 9 & 30.2 & 25.4 \\
\hline 10 & 35.9 & 39.7 \\
\hline 11 & 50.9 & 46.1 \\
\hline 12 & 47.5 & 45.3 \\
\hline 13 & 44.5 & 39.7 \\
\hline 14 & 31.3 & 37.0 \\
\hline 15 & 46.4 & 39.7 \\
\hline 16 & 54.3 & 54.5 \\
\hline 17 & 52.6 & 47.5 \\
\hline 18 & 58.8 & 59.1 \\
\hline 19 & 46.7 & 44.6 \\
\hline 20 & 49.6 & 56.2 \\
\hline 21 & 16.7 & \\
\hline 22 & 48.1 & 46.0 \\
\hline 23 & 37.8 & 41.0 \\
\hline 24 & 33.0 & 30.2 \\
\hline 25 & 44.8 & 39.2 \\
\hline 26 & 72.2 & 73.7 \\
\hline 27 & 29.3 & 27.2 \\
\hline 28 & 45.4 & 49.0 \\
\hline 29 & 58.0 & 51.7 \\
\hline
\end{tabular}




\begin{tabular}{|c|c|c|}
\hline 30 & 48.1 & 36.0 \\
\hline 31 & 48.1 & 60.7 \\
\hline 32 & 59.8 & 64.5 \\
\hline 33 & 51.7 & 49.4 \\
\hline 34 & 52.6 & 45.5 \\
\hline 35 & 48.7 & 36.3 \\
\hline 36 & 41.0 & 58.2 \\
\hline 37 & 50.7 & 47.6 \\
\hline 38 & 43.5 & 43.7 \\
\hline 39 & 61.6 & 66.8 \\
\hline 40 & 39.9 & 33.6 \\
\hline 41 & 56.8 & 62.0 \\
\hline 42 & 56.0 & 56.9 \\
\hline 43 & 42.2 & 41.9 \\
\hline 44 & 47.0 & 44.9 \\
\hline 45 & 52.3 & 56.1 \\
\hline 46 & 53.9 & 49.1 \\
\hline 47 & 57.0 & 64.3 \\
\hline 48 & 40.3 & 37.4 \\
\hline 49 & 61.1 & 63.4 \\
\hline 50 & 35.9 & 45.1 \\
\hline 51 & 56.0 & 48.6 \\
\hline 52 & 54.6 & 64.4 \\
\hline 53 & 57.3 & 46.1 \\
\hline 54 & 52.1 & 64.2 \\
\hline 55 & 77.8 & 76.8 \\
\hline 56 & 44.1 & 49.3 \\
\hline 57 & 50.4 & 44.3 \\
\hline 58 & 52.2 & 48.1 \\
\hline 59 & 43.8 & 46.4 \\
\hline 60 & 49.9 & 46.2 \\
\hline
\end{tabular}




\begin{tabular}{|c|c|c|}
\hline 61 & 46.9 & 46.4 \\
\hline 62 & 45.0 & 45.8 \\
\hline 63 & 45.2 & 45.3 \\
\hline 64 & 49.4 & 46.4 \\
\hline 65 & 46.7 & 43.6 \\
\hline 66 & & 49.3 \\
\hline 67 & & 47.9 \\
\hline 68 & 46.5 & 45.8 \\
\hline 69 & 35.9 & 37.8 \\
\hline 70 & 48.2 & 49.3 \\
\hline 71 & 72.0 & \\
\hline 72 & 26.7 & \\
\hline 73 & 54.5 & 58.8 \\
\hline 74 & & 17.1 \\
\hline 75 & & 63.2 \\
\hline 76 & & 56.1 \\
\hline 77 & 56.4 & 50.6 \\
\hline 78 & 47.5 & 45.6 \\
\hline 79 & 50.5 & 61.0 \\
\hline 80 & 60.2 & \\
\hline 81 & 66.6 & 57.8 \\
\hline 82 & 52.3 & 54.2 \\
\hline 83 & 49.1 & 49.3 \\
\hline 84 & & 48.4 \\
\hline 85 & & \\
\hline 86 & 59.7 & 64.8 \\
\hline 87 & 69.9 & \\
\hline 88 & & 54.6 \\
\hline 89 & & 63.0 \\
\hline 90 & 55.0 & 54.9 \\
\hline 91 & 50.8 & 54.9 \\
\hline
\end{tabular}




\begin{tabular}{|l|l|l|}
\hline 92 & 56.9 & 59.1 \\
\hline 93 & 42.8 & 40.6 \\
\hline 94 & 53.8 & 53.5 \\
\hline 95 & 49.3 & 51.4 \\
\hline 96 & 20.5 & -5.4 \\
\hline 97 & 48.3 & 52.6 \\
\hline 98 & & 91.6 \\
\hline 99 & & 69.7 \\
\hline
\end{tabular}


Table S4. Cartesian coordinates and total energies for $\operatorname{Ag}_{\mathrm{n}}(\mathrm{n}=1-99)$

$$
\begin{aligned}
& \text { B3LYP/aD } \\
& \begin{array}{llll}
\mathrm{Ag} 1 \\
\mathrm{E}=-146.971326 & \text { Hartree } \\
\mathrm{Ag} & 0.000000 & 0.000000 & 0.000000 \\
& & \\
\mathrm{Ag} 2 & \\
\mathrm{E}=-294.000593 & \text { Hartree } \\
\mathrm{Ag} & 0.000000 & 0.000000 & 1.292488 \\
\mathrm{Ag} & 0.000000 & 0.000000 & -1.292488
\end{array}
\end{aligned}
$$

Ag3

$\mathrm{E}=-440.994182$ Hartree

$\begin{array}{llll}\mathrm{Ag} & 0.000000 & 0.000000 & 1.676540\end{array}$

$\begin{array}{llll}\mathrm{Ag} & 0.000000 & 1.304549 & -0.838270\end{array}$

$\begin{array}{llll}\mathrm{Ag} & 0.000000 & -1.304549 & -0.838270\end{array}$

Ag3

$\mathrm{E}=-440.995795$ Hartree

$\begin{array}{llll}\mathrm{Ag} & 0.000000 & 0.000000 & 1.395757\end{array}$

$\begin{array}{llll}\mathrm{Ag} & 0.000000 & 1.627489 & -0.697878\end{array}$

$\begin{array}{llll}\mathrm{Ag} & 0.000000 & -1.627489 & -0.697878\end{array}$

$\operatorname{Ag} 4$

$\mathrm{E}=-588.030710$ Hartree

$\begin{array}{rrrr}\mathrm{Ag} & 1.158558 & 2.150309 & 0.000000 \\ \mathrm{Ag} & -1.158558 & -2.150309 & 0.000000 \\ \mathrm{Ag} & 1.158558 & -0.621572 & 0.000000 \\ \mathrm{Ag} & -1.158558 & 0.621572 & 0.000000\end{array}$

Ag5

$\mathrm{E}=-735.055111$ Hartree

$\begin{array}{llll}\text { Ag } & 0.000000 & 0.000000 & 0.979023\end{array}$

$\begin{array}{llll}\mathrm{Ag} & 0.000000 & 1.395145 & -1.444725\end{array}$

$\begin{array}{llll}\mathrm{Ag} & 0.000000 & -1.395145 & -1.444725\end{array}$

$\begin{array}{llll}\mathrm{Ag} & 0.000163 & 2.717945 & 0.955213\end{array}$

$\begin{array}{llll}\mathrm{Ag} & -0.000163 & -2.717945 & 0.955213\end{array}$

Ag6

$E=-882.102652$ Hartree

$\begin{array}{rrrr}\mathrm{Ag} & 0.000000 & -3.137733 & 0.000000 \\ \mathrm{Ag} & 0.000000 & 1.635287 & 0.000000 \\ \mathrm{Ag} & 1.416200 & -0.817644 & 0.000000 \\ \mathrm{Ag} & 2.717356 & 1.568867 & 0.000000 \\ \mathrm{Ag} & -2.717356 & 1.568867 & 0.000000\end{array}$




$$
\begin{array}{llll}
\mathrm{Ag} & -1.416200 & -0.817644 & 0.000000
\end{array}
$$

Ag7

$E=-1029.111508$ Hartree

$\begin{array}{rrrr}\mathrm{Ag} & 0.000263 & 0.742185 & 2.284646 \\ \mathrm{Ag} & -0.001337 & 2.403750 & 0.000000 \\ \mathrm{Ag} & 0.000263 & 0.742185 & -2.284646 \\ \mathrm{Ag} & 1.512376 & -0.000330 & 0.000000 \\ \mathrm{Ag} & -1.512091 & -0.000256 & 0.000000 \\ \mathrm{Ag} & 0.000263 & -1.943767 & -1.412035 \\ \mathrm{Ag} & 0.000263 & -1.943767 & 1.412035\end{array}$

Ag8

$E=-1176.156688$ Hartree

$\begin{array}{rrrr}\mathrm{Ag} & 0.904981 & -1.399327 & -0.598670 \\ \mathrm{Ag} & 1.952194 & 0.865707 & -1.874274 \\ \mathrm{Ag} & 1.290854 & -1.294002 & 2.175962 \\ \mathrm{Ag} & -1.786025 & -1.817394 & -1.258002 \\ \mathrm{Ag} & -1.214547 & -0.540162 & 1.169219 \\ \mathrm{Ag} & -1.455924 & 2.243769 & 0.959793 \\ \mathrm{Ag} & 1.111181 & 1.133759 & 0.784649 \\ \mathrm{Ag} & -0.802715 & 0.807651 & -1.358677\end{array}$

$\mathrm{Ag} 9$

$E=-1323.160966$ Hartree

$\begin{array}{rrrr}\mathrm{Ag} & -1.394732 & 0.000003 & 1.549679 \\ \mathrm{Ag} & 2.321067 & -1.476313 & -0.786097 \\ \mathrm{Ag} & -0.000004 & -2.280359 & 0.626655 \\ \mathrm{Ag} & 2.321074 & 1.476303 & -0.786096 \\ \mathrm{Ag} & 1.394730 & 0.000001 & 1.549686 \\ \mathrm{Ag} & -2.321077 & -1.476304 & -0.786089 \\ \mathrm{Ag} & -2.321062 & 1.476317 & -0.786102 \\ \mathrm{Ag} & -0.000001 & -0.000006 & -1.208294 \\ \mathrm{Ag} & 0.000005 & 2.280357 & 0.626659\end{array}$

Ag9

$E=-1323.162340$ Hartree

$\begin{array}{lrrr}\mathrm{Ag} & -0.601887 & 1.483998 & -0.141158 \\ \mathrm{Ag} & 1.364976 & -0.126257 & -1.452804 \\ \mathrm{Ag} & 1.365853 & 0.139502 & 1.450258 \\ \mathrm{Ag} & 2.079023 & 2.299286 & -0.209158 \\ \mathrm{Ag} & -3.100475 & -0.004016 & 0.001961 \\ \mathrm{Ag} & -1.323196 & 0.204597 & 2.276754 \\ \mathrm{Ag} & -0.579143 & -1.494837 & 0.141273 \\ \mathrm{Ag} & -1.324351 & -0.229442 & -2.274752 \\ \mathrm{Ag} & 2.119200 & -2.272831 & 0.207627\end{array}$




\begin{tabular}{|c|c|c|c|}
\hline \multicolumn{4}{|l|}{ Ag9 } \\
\hline \\
\hline $\mathrm{Ag}$ & 1.931874 & 1.999788 & 0.000098 \\
\hline $\mathrm{Ag}$ & 0.985052 & 0.083837 & -1.876017 \\
\hline $\mathrm{Ag}$ & -0.763138 & $1.4 \mathrm{C}$ & -0.000259 \\
\hline $\mathrm{Ag}$ & 2.993604 & -0.68 & -0.000201 \\
\hline $\mathrm{Ag}$ & 0.985072 & 0.083945 & 1.875973 \\
\hline $\mathrm{Ag}$ & -1.587762 & -0.813043 & 1.483674 \\
\hline $\mathrm{Ag}$ & 0.509366 & -2.035588 & 0.000290 \\
\hline $\mathrm{Ag}$ & -1.587635 & -0.813644 & -1.483323 \\
\hline $\mathrm{Ag}$ & -3.466433 & 0.688050 & -0.000236 \\
\hline
\end{tabular}

Ag10

$E=-1470.190379$ Hartree

$\begin{array}{llll}\mathrm{Ag} & 2.307486 & 0.009004 & -0.158769\end{array}$

$\begin{array}{llll}\text { Ag } & 0.164541 & -1.775200 & 0.165773\end{array}$

$\begin{array}{llll}\mathrm{Ag} & 0.150021 & 1.775721 & 0.168878\end{array}$

$\begin{array}{llll}\mathrm{Ag} & 0.661619 & 0.000294 & 2.371413\end{array}$

$\begin{array}{llll}\mathrm{Ag} & -1.816204 & -0.008490 & 1.206669\end{array}$

$\begin{array}{llll}\text { Ag } & -2.493816 & 2.354858 & -0.387442\end{array}$

$\begin{array}{llll}\mathrm{Ag} & 2.583473 & 2.659802 & -0.705912\end{array}$

$\begin{array}{llll}\mathrm{Ag} & -1.690889 & -0.003497 & -1.563779\end{array}$

$\begin{array}{llll}\mathrm{Ag} & 2.607719 & -2.640118 & -0.701316\end{array}$

Ag $\quad-2.473950 \quad-2.372375 \quad-0.395514$

Ag11

$\mathrm{E}=-1617.222941$ Hartree

$\begin{array}{llll}\text { Ag } & 0.000070 & -2.586236 & -0.008360\end{array}$

$\begin{array}{lllll}\mathrm{Ag} & 1.414287 & -0.621251 & -1.458923\end{array}$

$\begin{array}{llll}\mathrm{Ag} & 3.821737 & -0.876859 & -0.002433\end{array}$

$\begin{array}{llll}\mathrm{Ag} & 1.416260 & -0.639583 & 1.449166\end{array}$

$\begin{array}{llll}\mathrm{Ag} & -1.416249 & -0.639631 & 1.449141\end{array}$

$\begin{array}{llll}\mathrm{Ag} & -0.000023 & 1.825037 & 1.422946\end{array}$

$\begin{array}{llll}\mathrm{Ag} & 2.505301 & 1.590783 & 0.005193\end{array}$

$\begin{array}{llll}\mathrm{Ag} & -0.000023 & 1.855173 & -1.400588\end{array}$

$\begin{array}{llll}\mathrm{Ag} & -1.414285 & -0.621304 & -1.458908\end{array}$

$\begin{array}{llll}\mathrm{Ag} & -2.505326 & 1.590755 & 0.005191\end{array}$

Ag $\quad-3.821750 \quad-0.876883 \quad-0.002424$

Ag12

$\mathrm{E}=-1764.258140$ Hartree

$\begin{array}{llll}\mathrm{Ag} & 2.920990 & -1.815754 & -0.011252\end{array}$

$\begin{array}{llll}\text { Ag } & -2.091570 & -2.100565 & 0.027884\end{array}$

$\begin{array}{llll}\text { Ag } & 0.425859 & -2.032091 & 1.429367\end{array}$

$\begin{array}{llll}\mathrm{Ag} & -3.891492 & -0.003057 & -0.009968\end{array}$ 


$\begin{array}{lrrr}\mathrm{Ag} & 1.805186 & 0.337091 & 1.453956 \\ \mathrm{Ag} & 0.407230 & -2.048794 & -1.433705 \\ \mathrm{Ag} & 1.752869 & 0.339274 & -1.462594 \\ \mathrm{Ag} & -1.201315 & 0.254723 & 1.426446 \\ \mathrm{Ag} & 3.231811 & 2.188853 & -0.022585 \\ \mathrm{Ag} & 0.339542 & 2.279037 & 0.023231 \\ \mathrm{Ag} & -2.477937 & 2.363407 & -0.008202 \\ \mathrm{Ag} & -1.221174 & 0.237876 & -1.412577\end{array}$

Ag13

$\mathrm{E}=-1911.283911$ Hartree

$\begin{array}{lrrr}\mathrm{Ag} & -1.196778 & 0.243685 & 1.171356 \\ \mathrm{Ag} & -2.884029 & 2.290275 & 0.272120 \\ \mathrm{Ag} & -2.081931 & -2.467099 & 1.163354 \\ \mathrm{Ag} & -1.485419 & 0.884620 & -1.749954 \\ \mathrm{Ag} & -3.487140 & -0.501674 & -0.301335 \\ \mathrm{Ag} & -0.000004 & 2.621302 & -0.000001 \\ \mathrm{Ag} & 1.196774 & 0.243680 & -1.171346 \\ \mathrm{Ag} & 0.794877 & -1.760464 & 1.205829 \\ \mathrm{Ag} & -0.794870 & -1.760477 & -1.205829 \\ \mathrm{Ag} & 3.487137 & -0.501662 & 0.301332 \\ \mathrm{Ag} & 1.485418 & 0.884633 & 1.749952 \\ \mathrm{Ag} & 2.081945 & -2.467100 & -1.163346 \\ \mathrm{Ag} & 2.884020 & 2.290281 & -0.272132\end{array}$

Ag14

$\mathrm{E}=-2058.294184$ Hartree

$\begin{array}{lrrr}\mathrm{Ag} & 0.393827 & 0.007244 & 0.001682 \\ \mathrm{Ag} & -2.030091 & -0.159173 & 1.753561 \\ \mathrm{Ag} & -2.011457 & 1.595493 & -0.782891 \\ \mathrm{Ag} & -2.023542 & -1.475391 & -1.016882 \\ \mathrm{Ag} & -4.185046 & 0.014089 & -0.012712 \\ \mathrm{Ag} & 0.319642 & -1.242031 & -2.568194 \\ \mathrm{Ag} & 0.308677 & -2.846767 & -0.221356 \\ \mathrm{Ag} & 2.672918 & -1.563281 & 0.761539 \\ \mathrm{Ag} & 2.666612 & 1.441684 & 0.992353 \\ \mathrm{Ag} & 2.689726 & 0.118969 & -1.717640 \\ \mathrm{Ag} & 0.327806 & 1.619989 & -2.359816 \\ \mathrm{Ag} & 0.298724 & -1.616941 & 2.364482 \\ \mathrm{Ag} & 0.300637 & 2.871206 & 0.216004 \\ \mathrm{Ag} & 0.271568 & 1.234911 & 2.589870\end{array}$

Ag15

$E=-2205.319202$ Hartree

$\begin{array}{rrrr}\mathrm{Ag} & -1.256455 & -2.268221 & 0.028732 \\ \mathrm{Ag} & 0.941549 & 1.296163 & -2.422986\end{array}$ 


$\begin{array}{lrrr}\mathrm{Ag} & -1.918524 & 0.130534 & 1.607656 \\ \mathrm{Ag} & -0.747190 & 2.441326 & -0.019162 \\ \mathrm{Ag} & -3.824603 & -1.173027 & 0.013262 \\ \mathrm{Ag} & -3.476105 & 1.801487 & -0.015450 \\ \mathrm{Ag} & -1.921334 & 0.100266 & -1.608519 \\ \mathrm{Ag} & 2.247702 & 2.234901 & -0.028608 \\ \mathrm{Ag} & 0.633038 & -0.050772 & 0.000481 \\ \mathrm{Ag} & 1.823375 & -2.536171 & 0.020195 \\ \mathrm{Ag} & 0.170369 & -1.507453 & -2.368279 \\ \mathrm{Ag} & 0.956609 & 1.355707 & 2.387912 \\ \mathrm{Ag} & 3.092537 & -0.201729 & -1.444742 \\ \mathrm{Ag} & 0.183511 & -1.447005 & 2.406575 \\ \mathrm{Ag} & 3.095522 & -0.176007 & 1.442932\end{array}$

Ag16

$E=-2352.372047$ Hartree

$\begin{array}{lrrc}\mathrm{Ag} & -2.798257 & 1.860578 & 0.623601 \\ \mathrm{Ag} & -2.457131 & -0.042773 & -1.633174 \\ \mathrm{Ag} & -1.201380 & 0.431914 & 2.448005 \\ \mathrm{Ag} & -0.484960 & -2.058013 & 1.228538 \\ \mathrm{Ag} & 1.462252 & -0.605783 & 2.733330 \\ \mathrm{Ag} & -3.178000 & -0.901190 & 0.944056 \\ \mathrm{Ag} & -2.160788 & -2.851316 & -0.833918 \\ \mathrm{Ag} & 3.421897 & 0.586352 & 0.908800 \\ \mathrm{Ag} & 2.537783 & -2.069344 & 0.571177 \\ \mathrm{Ag} & 1.997195 & 2.453102 & -0.809619 \\ \mathrm{Ag} & -1.032137 & 2.324645 & -1.509762 \\ \mathrm{Ag} & 0.607347 & -2.356111 & -1.410304 \\ \mathrm{Ag} & 2.697527 & -0.343113 & -1.701480 \\ \mathrm{Ag} & 0.174575 & 0.193225 & -2.757602 \\ \mathrm{Ag} & 0.631688 & 0.336562 & 0.186251 \\ \mathrm{Ag} & -0.217611 & 3.041265 & 1.012100\end{array}$

Ag17

$\mathrm{E}=-2499.408081$ Hartree

$\begin{array}{lrrr}\mathrm{Ag} & -0.291273 & -0.505599 & -2.890404 \\ \mathrm{Ag} & -0.012821 & 0.331355 & -0.002231 \\ \mathrm{Ag} & 1.853049 & -2.354885 & 1.466842 \\ \mathrm{Ag} & 0.305114 & -0.511270 & 2.886323 \\ \mathrm{Ag} & 2.540067 & 0.652165 & 1.183289 \\ \mathrm{Ag} & -2.146239 & 0.643731 & 1.837071 \\ \mathrm{Ag} & -0.926973 & -2.373771 & 1.192376 \\ \mathrm{Ag} & -3.520193 & -1.441539 & 0.527913 \\ \mathrm{Ag} & 2.130931 & 0.697496 & -1.827384 \\ \mathrm{Ag} & 0.252731 & 2.202297 & 2.245166 \\ \mathrm{Ag} & 1.446393 & 2.989208 & -0.164001\end{array}$




$\begin{array}{lrrr}\mathrm{Ag} & 0.983126 & -2.343728 & -1.199329 \\ \mathrm{Ag} & 3.556696 & -1.362193 & -0.532632 \\ \mathrm{Ag} & -1.795595 & -2.386795 & -1.474435 \\ \mathrm{Ag} & -1.512543 & 2.954282 & 0.176677 \\ \mathrm{Ag} & -0.300106 & 2.205993 & -2.236508 \\ \mathrm{Ag} & -2.562366 & 0.603254 & -1.188734\end{array}$

Ag18

$E=-2646.462108$ Hartree

$\begin{array}{lrrr}\mathrm{Ag} & -0.000020 & 3.254869 & -1.241085 \\ \mathrm{Ag} & 0.000005 & 0.742237 & 0.050449 \\ \mathrm{Ag} & 2.350035 & 0.050831 & 1.613967 \\ \mathrm{Ag} & -1.516848 & 2.829439 & 1.233279 \\ \mathrm{Ag} & -0.000012 & 1.011323 & 2.843440 \\ \mathrm{Ag} & 2.377500 & 1.471228 & -1.299368 \\ \mathrm{Ag} & -0.000008 & 0.862841 & -2.787111 \\ \mathrm{Ag} & 1.516844 & 2.829435 & 1.233271 \\ \mathrm{Ag} & 3.944987 & -0.774984 & -0.701360 \\ \mathrm{Ag} & -1.440565 & -1.331662 & -1.749526 \\ \mathrm{Ag} & -2.350037 & 0.050816 & 1.613963 \\ \mathrm{Ag} & 1.440577 & -1.331659 & -1.749528 \\ \mathrm{Ag} & -2.377517 & 1.471214 & -1.299362 \\ \mathrm{Ag} & -3.944976 & -0.775015 & -0.701361 \\ \mathrm{Ag} & 0.000014 & -3.412002 & -0.512174 \\ \mathrm{Ag} & 0.000001 & -1.706093 & 1.931416 \\ \mathrm{Ag} & -2.392571 & -2.621420 & 0.760545 \\ \mathrm{Ag} & 2.392591 & -2.621399 & 0.760546\end{array}$

Ag19

$E=-2793.491101$ Hartree

$\begin{array}{lrrr}\mathrm{Ag} & -4.058156 & -0.161980 & -1.467984 \\ \mathrm{Ag} & 0.000013 & 0.924508 & 2.473443 \\ \mathrm{Ag} & -0.000004 & -0.842537 & 0.225530 \\ \mathrm{Ag} & -2.443442 & 0.511508 & 0.870200 \\ \mathrm{Ag} & 0.000023 & -2.554832 & -1.991008 \\ \mathrm{Ag} & -1.435833 & -1.634318 & 2.519986 \\ \mathrm{Ag} & 2.423021 & -2.160860 & -0.277672 \\ \mathrm{Ag} & -2.423022 & -2.160856 & -0.277687 \\ \mathrm{Ag} & -0.000010 & -3.561435 & 0.844686 \\ \mathrm{Ag} & 1.435823 & -1.634327 & 2.519983 \\ \mathrm{Ag} & 1.404848 & -0.030046 & -2.214242 \\ \mathrm{Ag} & 2.443440 & 0.511510 & 0.870199 \\ \mathrm{Ag} & -0.000007 & 1.978305 & -0.725902 \\ \mathrm{Ag} & 2.731680 & 2.379936 & -1.224889 \\ \mathrm{Ag} & -1.404850 & -0.030056 & -2.214240 \\ \mathrm{Ag} & 1.467362 & 3.123765 & 1.381227\end{array}$




$\begin{array}{lrrr}\mathrm{Ag} & -1.467358 & 3.123760 & 1.381234 \\ \mathrm{Ag} & -2.731683 & 2.379937 & -1.224887 \\ \mathrm{Ag} & 4.058155 & -0.161981 & -1.467979\end{array}$

\begin{tabular}{|c|c|c|c|}
\hline \\
\hline \multicolumn{4}{|c|}{$\begin{array}{l}\text { Ag20 } \\
E=-2940.550040 \text { Hartree }\end{array}$} \\
\hline $\mathrm{Ag}$ & -1.105884 & 1.105884 & 1.105884 \\
\hline $\mathrm{Ag}$ & -0.981190 & -0.981190 & 3.074906 \\
\hline $\mathrm{Ag}$ & 0.981190 & 3.074906 & 0.981190 \\
\hline $\mathrm{Ag}$ & -3.074906 & 0.981190 & -0.981190 \\
\hline $\mathrm{Ag}$ & 0.981190 & & 906 \\
\hline $\mathrm{Ag}$ & -3.074906 & -0.981190 & 0.981190 \\
\hline $\mathrm{Ag}$ & -0.981190 & & 1190 \\
\hline $\mathrm{Ag}$ & 2.954125 & 2.954125 & 2.954125 \\
\hline $\mathrm{Ag}$ & -2.954125 & & -2.954125 \\
\hline $\mathrm{Ag}$ & -2.954125 & -2.954125 & 2.954125 \\
\hline $\mathrm{Ag}$ & 3.074906 & 0.98 & 1190 \\
\hline $\mathrm{Ag}$ & 1.105884 & -1.10 & 1.105884 \\
\hline $\mathrm{Ag}$ & 3.074906 & -0.9 & 190 \\
\hline $\mathrm{Ag}$ & -0.981190 & -3.074906 & 0.981190 \\
\hline $\mathrm{Ag}$ & 0.981190 & -3.074906 & -0.981190 \\
\hline $\mathrm{Ag}$ & 2.954125 & -2.954125 & -2.954125 \\
\hline $\mathrm{Ag}$ & 1.105884 & 1.105884 & -1.105884 \\
\hline $\mathrm{Ag}$ & 0.981190 & -0.981190 & -3.074906 \\
\hline $\mathrm{Ag}$ & -0.981190 & 0.981190 & -3.074906 \\
\hline $\mathrm{Ag}$ & -1.105884 & -1.105884 & -1.105884 \\
\hline
\end{tabular}




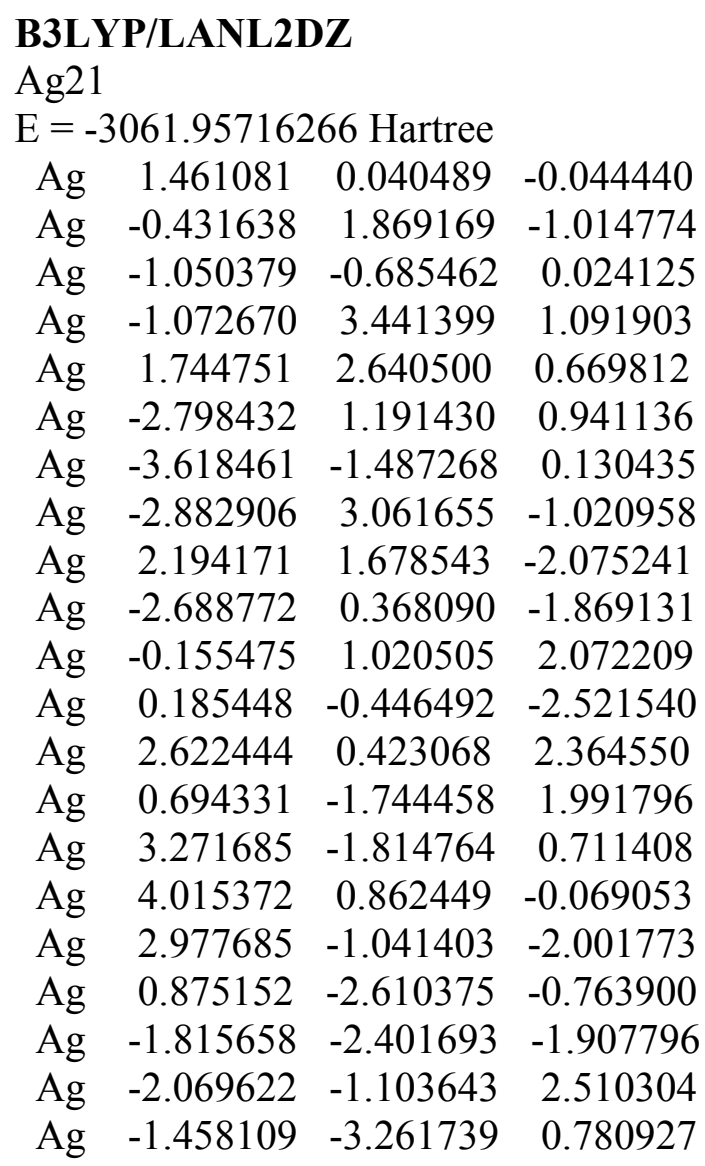

$\begin{array}{lrrr}\mathrm{Ag} 22 & & & \\ \mathrm{E}=-3207.77436618 & \text { Hartree } & \\ \mathrm{Ag} & 1.283218 & -2.545468 & -0.777311 \\ \mathrm{Ag} & -0.781969 & -0.772190 & 0.083694 \\ \mathrm{Ag} & 4.146317 & 1.177816 & -0.207296 \\ \mathrm{Ag} & 1.140794 & -1.703035 & 1.972535 \\ \mathrm{Ag} & 3.663753 & -1.553405 & 0.596426 \\ \mathrm{Ag} & -0.939602 & -3.381413 & 0.840637 \\ \mathrm{Ag} & 2.909513 & 0.618916 & 2.277688 \\ \mathrm{Ag} & -1.627633 & -1.320704 & 2.619902 \\ \mathrm{Ag} & -3.292036 & -1.746372 & 0.258180 \\ \mathrm{Ag} & -2.598771 & 0.938709 & 1.202927 \\ \mathrm{Ag} & -2.485736 & 0.082873 & -1.872401 \\ \mathrm{Ag} & -3.038016 & 2.678087 & -0.846119 \\ \mathrm{Ag} & -1.430903 & -2.586407 & -1.821188 \\ \mathrm{Ag} & -4.755687 & 0.533462 & -0.430417 \\ \mathrm{Ag} & -0.409308 & 1.812942 & -0.982220 \\ \mathrm{Ag} & 0.370698 & -0.456320 & -2.513227 \\ \mathrm{Ag} & 1.668277 & 0.143731 & -0.080143 \\ \mathrm{Ag} & 3.206689 & -0.807362 & -2.094848 \\ \mathrm{Ag} & 0.094709 & 0.980056 & 2.122696\end{array}$




$\begin{array}{lrrr}\mathrm{Ag} & 2.185117 & 1.836719 & -2.134663 \\ \mathrm{Ag} & -1.075485 & 3.311827 & 1.159883 \\ \mathrm{Ag} & 1.766062 & 2.757537 & 0.625265\end{array}$

Ag23

$\mathrm{E}=-3353.57023864$ Hartree

$\begin{array}{lrrr}\mathrm{Ag} & -2.417500 & -1.570717 & 2.309989 \\ \mathrm{Ag} & -2.351593 & -3.275936 & -0.000069 \\ \mathrm{Ag} & 0.000044 & -0.000023 & 2.423650 \\ \mathrm{Ag} & -2.450850 & 1.248142 & 1.455470 \\ \mathrm{Ag} & -1.288189 & -0.836989 & 0.000027 \\ \mathrm{Ag} & -2.450995 & 1.248158 & -1.455285 \\ \mathrm{Ag} & -0.080719 & 1.534145 & 0.000019 \\ \mathrm{Ag} & -3.948886 & -0.817446 & 0.000048 \\ \mathrm{Ag} & -1.661243 & 3.674483 & 0.000130 \\ \mathrm{Ag} & -0.151337 & 2.878869 & 2.310014 \\ \mathrm{Ag} & 1.266490 & 3.828484 & -0.000084 \\ \mathrm{Ag} & 2.569005 & -1.308267 & 2.310012 \\ \mathrm{Ag} & 4.012846 & -0.398535 & -0.000070 \\ \mathrm{Ag} & 0.144571 & -2.746601 & 1.455301 \\ \mathrm{Ag} & 2.568951 & -1.308312 & -2.309990 \\ \mathrm{Ag} & 1.368918 & -0.697143 & -0.000055 \\ \mathrm{Ag} & 2.682406 & -3.011025 & 0.000027 \\ \mathrm{Ag} & 2.306415 & 1.498487 & 1.455241 \\ \mathrm{Ag} & 0.144565 & -2.746620 & -1.455358 \\ \mathrm{Ag} & 2.306314 & 1.498459 & -1.455388 \\ \mathrm{Ag} & -0.000037 & 0.000032 & -2.423657 \\ \mathrm{Ag} & -0.151623 & 2.878984 & -2.310008 \\ \mathrm{Ag} & -2.417552 & -1.570629 & -2.309966\end{array}$

Ag24

$\mathrm{E}=-3499.36451558$ Hartree

$\begin{array}{lrrr}\mathrm{Ag} & -1.349177 & 0.987216 & 2.412896 \\ \mathrm{Ag} & -2.924914 & -1.487273 & 1.938694 \\ \mathrm{Ag} & -1.355308 & -2.939924 & -0.000039 \\ \mathrm{Ag} & -3.923508 & 0.857943 & 0.804662 \\ \mathrm{Ag} & -0.180150 & -1.662709 & 2.412910 \\ \mathrm{Ag} & -3.701654 & -1.558581 & -0.804157 \\ \mathrm{Ag} & 0.768574 & -3.190069 & -1.938735 \\ \mathrm{Ag} & 0.894829 & -1.266130 & 0.000075 \\ \mathrm{Ag} & 3.200430 & -2.426334 & -0.804736 \\ \mathrm{Ag} & 2.750875 & -1.789778 & 1.938315 \\ \mathrm{Ag} & 1.218882 & -3.826890 & 0.804173 \\ \mathrm{Ag} & -1.147349 & -1.216733 & -2.412913 \\ \mathrm{Ag} & -1.543878 & -0.141808 & -0.000003 \\ \mathrm{Ag} & -3.147580 & 0.929424 & -1.938278\end{array}$




$\begin{array}{lrrr}\mathrm{Ag} & 0.649120 & 1.407987 & -0.000020 \\ \mathrm{Ag} & -1.868344 & 2.643763 & 0.000226 \\ \mathrm{Ag} & 2.704935 & 2.968941 & 0.804180 \\ \mathrm{Ag} & 1.627006 & -0.384782 & -2.412878 \\ \mathrm{Ag} & 3.223752 & 0.296256 & -0.000219 \\ \mathrm{Ag} & -0.480584 & 1.601783 & -2.412637 \\ \mathrm{Ag} & 2.378231 & 2.261066 & -1.938699 \\ \mathrm{Ag} & 0.500930 & 3.984987 & -0.804199 \\ \mathrm{Ag} & 1.530161 & 0.674789 & 2.412708 \\ \mathrm{Ag} & 0.174720 & 3.276855 & 1.938675\end{array}$

\begin{tabular}{|c|c|c|c|}
\hline \\
\hline \multicolumn{4}{|c|}{$\begin{array}{l}\text { Ag25 } \\
E=-3645.17186540 \text { Hartree }\end{array}$} \\
\hline $\mathrm{Ag}$ & & & -2.394764 \\
\hline $\mathrm{Ag}$ & & & -2.109686 \\
\hline $\mathrm{Ag}$ & & & \\
\hline $\mathrm{Ag}$ & -1.295 & & \\
\hline $\mathrm{Ag}$ & & & \\
\hline $\mathrm{Ag}$ & -1.3 & & \\
\hline $\mathrm{Ag}$ & & & \\
\hline $\mathrm{Ag}$ & -3.1 & & \\
\hline $\mathrm{Ag}$ & & & \\
\hline $\mathrm{Ag}$ & -0.2 & & \\
\hline $\mathrm{Ag}$ & -2.7 & & \\
\hline $\mathrm{Ag}$ & -3.1 & & \\
\hline $\mathrm{Ag}$ & -2.0 & & \\
\hline $\mathrm{Ag}$ & & & \\
\hline $\mathrm{Ag}$ & 3.9 & -0 . & \\
\hline $\mathrm{Ag}$ & 0.6 & & \\
\hline $\mathrm{Ag}$ & 3.4 & -2. & 67 \\
\hline $\mathrm{Ag}$ & & & \\
\hline $\mathrm{Ag}$ & & & \\
\hline $\mathrm{Ag}$ & 1.3 & & \\
\hline $\mathrm{Ag}$ & 0.44 & 4.2 & \\
\hline $\mathrm{Ag}$ & -1.38 & & \\
\hline $\mathrm{Ag}$ & 2.983955 & & 1.918716 \\
\hline $\mathrm{Ag}$ & -0.000045 & -0.0 & \\
\hline $\mathrm{Ag}$ & 1.409605 & -2.335226 & 2.338087 \\
\hline
\end{tabular}

Ag26

$E=-3791.03194027$ Hartree

$\begin{array}{lrrr}\mathrm{Ag} & 2.156943 & -0.256328 & -0.258843 \\ \mathrm{Ag} & 4.153473 & 0.206770 & 1.500017 \\ \mathrm{Ag} & 2.819226 & -0.592996 & -2.851669 \\ \mathrm{Ag} & 4.222720 & 1.214723 & -1.171937 \\ \mathrm{Ag} & 0.000029 & -1.787523 & 0.575996\end{array}$




$\begin{array}{lrrr}\mathrm{Ag} & 4.415936 & -1.631150 & -0.666139 \\ \mathrm{Ag} & 1.446013 & 0.231973 & 2.457518 \\ \mathrm{Ag} & 1.495103 & 1.852529 & -1.998812 \\ \mathrm{Ag} & 2.404991 & 2.400047 & 0.724150 \\ \mathrm{Ag} & 0.000053 & 1.126370 & 0.237046 \\ \mathrm{Ag} & -0.000011 & 2.768180 & 2.348807 \\ \mathrm{Ag} & -1.495145 & 1.852537 & -1.998806 \\ \mathrm{Ag} & 0.000045 & 3.727008 & -0.347278 \\ \mathrm{Ag} & -2.404945 & 2.399998 & 0.724147 \\ \mathrm{Ag} & -0.000079 & -0.665181 & -2.104225 \\ \mathrm{Ag} & -1.445916 & 0.232031 & 2.457538 \\ \mathrm{Ag} & 1.870015 & -2.763506 & -1.278150 \\ \mathrm{Ag} & -1.870105 & -2.763522 & -1.278088 \\ \mathrm{Ag} & -2.156887 & -0.256365 & -0.258721 \\ \mathrm{Ag} & -2.819288 & -0.592927 & -2.851600 \\ \mathrm{Ag} & 0.000026 & -2.030000 & 3.227251 \\ \mathrm{Ag} & 2.588097 & -2.231581 & 1.574880 \\ \mathrm{Ag} & -4.222751 & 1.214781 & -1.171922 \\ \mathrm{Ag} & -2.588086 & -2.231502 & 1.574855 \\ \mathrm{Ag} & -4.153428 & 0.206752 & 1.500081 \\ \mathrm{Ag} & -4.416029 & -1.631121 & -0.666097\end{array}$

Ag27

$\mathrm{E}=-3936.81821045$ Hartree

$\begin{array}{lrrr}\mathrm{Ag} & -1.540135 & -0.007382 & 0.027989 \\ \mathrm{Ag} & -4.140731 & -0.730728 & 0.203568 \\ \mathrm{Ag} & -2.247227 & -1.408608 & -2.270753 \\ \mathrm{Ag} & -3.638893 & 1.987395 & 0.717218 \\ \mathrm{Ag} & -1.096917 & 2.313680 & 1.937591 \\ \mathrm{Ag} & -3.347772 & 1.085301 & -1.869449 \\ \mathrm{Ag} & 0.708695 & 1.532337 & -0.040840 \\ \mathrm{Ag} & -2.859491 & 0.195190 & 2.606512 \\ \mathrm{Ag} & 1.899368 & 1.987585 & 2.283067 \\ \mathrm{Ag} & -0.077593 & -0.245708 & 2.383877 \\ \mathrm{Ag} & -2.018309 & -2.250898 & 1.706061 \\ \mathrm{Ag} & -3.423737 & -3.228189 & -0.515508 \\ \mathrm{Ag} & -0.715801 & -2.949798 & -0.579511 \\ \mathrm{Ag} & 0.700355 & -1.584311 & -2.620127 \\ \mathrm{Ag} & -1.550657 & 2.983376 & -0.793233 \\ \mathrm{Ag} & -0.567687 & 0.917190 & -2.491899 \\ \mathrm{Ag} & 2.270855 & 0.880008 & -2.180133 \\ \mathrm{Ag} & 1.268607 & -1.070736 & -0.004331 \\ \mathrm{Ag} & 0.632350 & 4.157636 & 0.685816 \\ \mathrm{Ag} & 2.998157 & 3.048331 & -0.259397 \\ \mathrm{Ag} & 3.533093 & 0.423601 & 0.368547 \\ \mathrm{Ag} & 3.444986 & -1.562930 & -1.740085\end{array}$




$$
\begin{array}{lrrr}
\mathrm{Ag} & 0.853859 & -2.967677 & 1.843394 \\
\mathrm{Ag} & 2.553145 & -0.739827 & 2.695089 \\
\mathrm{Ag} & 1.881050 & -3.681946 & -0.894952 \\
\mathrm{Ag} & 3.573710 & -2.465527 & 0.882129 \\
\mathrm{Ag} & 0.906717 & 3.382634 & -2.080640
\end{array}
$$

$$
\begin{array}{lrcc}
\multicolumn{4}{l}{\mathrm{Ag} 28} \\
\mathrm{E}=-4082.62676985 & \text { Hartree } & \\
\mathrm{Ag} & -1.052401 & -1.214613 & -0.477265 \\
\mathrm{Ag} & -3.625461 & -2.083066 & -0.266751 \\
\mathrm{Ag} & -2.836098 & 0.341328 & -1.764514 \\
\mathrm{Ag} & -0.463591 & -0.295320 & -3.312248 \\
\mathrm{Ag} & 1.126009 & 0.271903 & -1.211883 \\
\mathrm{Ag} & -2.168838 & -2.405817 & -2.657509 \\
\mathrm{Ag} & 1.641636 & 1.789737 & -3.414073 \\
\mathrm{Ag} & 3.709846 & 1.002608 & -1.669185 \\
\mathrm{Ag} & -0.816604 & 2.374947 & -2.228502 \\
\mathrm{Ag} & 2.427940 & -0.913564 & -3.290053 \\
\mathrm{Ag} & 0.692982 & -2.642687 & -1.951822 \\
\mathrm{Ag} & 2.932391 & -1.574058 & -0.444227 \\
\mathrm{Ag} & 0.874156 & -3.112188 & 0.907638 \\
\mathrm{Ag} & -3.136996 & 0.260562 & 1.168346 \\
\mathrm{Ag} & -1.476557 & -3.903224 & -0.372735 \\
\mathrm{Ag} & 3.040760 & 0.972125 & 1.040381 \\
\mathrm{Ag} & 3.038806 & -1.682566 & 2.342895 \\
\mathrm{Ag} & 0.790676 & -0.465043 & 1.403180 \\
\mathrm{Ag} & 1.868853 & 0.480628 & 3.718921 \\
\mathrm{Ag} & 0.805781 & 2.491701 & 2.101326 \\
\mathrm{Ag} & -1.035182 & 0.446336 & 3.162742 \\
\mathrm{Ag} & 0.555175 & -2.011071 & 3.633351 \\
\mathrm{Ag} & -0.864326 & 1.407770 & 0.285874 \\
\mathrm{Ag} & 1.632035 & 2.859664 & -0.657542 \\
\mathrm{Ag} & -1.689630 & -2.122408 & 1.978311 \\
\mathrm{Ag} & -2.127932 & 2.838799 & 2.228733 \\
\mathrm{Ag} & -3.123200 & 2.763017 & -0.406740 \\
\mathrm{Ag} & -0.720230 & 4.124500 & 0.153349
\end{array}
$$

$$
\begin{aligned}
& \text { Ag29 } \\
& \begin{array}{cccc}
\mathrm{E}=-4228.46109670 & \text { Hartree } & \\
\mathrm{Ag} & 0.000001 & 2.210354 & 2.724896 \\
\mathrm{Ag} & 1.352423 & 3.958199 & 0.746032 \\
\mathrm{Ag} & 0.000020 & 3.665163 & -1.684934 \\
\mathrm{Ag} & -2.330431 & 2.298984 & -1.382232 \\
\mathrm{Ag} & -2.200872 & -0.185308 & -0.266368 \\
\mathrm{Ag} & -4.181327 & -1.886556 & -1.054457 \\
\mathrm{Ag} & -2.834295 & 1.816738 & 1.472460
\end{array}
\end{aligned}
$$




$\begin{array}{lrrr}\mathrm{Ag} & -4.655588 & 0.876150 & -0.711193 \\ \mathrm{Ag} & -2.887564 & -0.094785 & -2.856105 \\ \mathrm{Ag} & -1.468595 & -2.403168 & -1.857922 \\ \mathrm{Ag} & -4.158312 & -0.713092 & 1.570014 \\ \mathrm{Ag} & -0.000008 & 0.119759 & -2.190913 \\ \mathrm{Ag} & 0.000043 & 1.504800 & 0.194681 \\ \mathrm{Ag} & -1.352418 & 3.958184 & 0.746057 \\ \mathrm{Ag} & 2.834281 & 1.816720 & 1.472451 \\ \mathrm{Ag} & -0.000004 & -1.464482 & 0.310377 \\ \mathrm{Ag} & 2.200843 & -0.185317 & -0.266376 \\ \mathrm{Ag} & 2.346775 & -2.785876 & 0.932764 \\ \mathrm{Ag} & -1.411259 & -0.373418 & 2.461049 \\ \mathrm{Ag} & -2.346720 & -2.785796 & 0.932755 \\ \mathrm{Ag} & 1.411278 & -0.373351 & 2.461086 \\ \mathrm{Ag} & -0.000023 & -2.918000 & 2.581168 \\ \mathrm{Ag} & -0.000016 & -4.133452 & -0.043389 \\ \mathrm{Ag} & 4.158316 & -0.713050 & 1.570047 \\ \mathrm{Ag} & 1.468635 & -2.403203 & -1.857911 \\ \mathrm{Ag} & 4.181266 & -1.886557 & -1.054514 \\ \mathrm{Ag} & 2.887569 & -0.094777 & -2.856072 \\ \mathrm{Ag} & 4.655619 & 0.876150 & -0.711203 \\ \mathrm{Ag} & 2.330362 & 2.298986 & -1.382247\end{array}$

Ag30

$E=-4374.27723755$ Hartree

$\begin{array}{lrrr}\mathrm{Ag} & 1.207156 & -3.865764 & 1.212708 \\ \mathrm{Ag} & 3.411853 & -2.784873 & -0.025705 \\ \mathrm{Ag} & 1.325642 & -3.831578 & -1.593957 \\ \mathrm{Ag} & 2.106644 & -1.409874 & 2.157001 \\ \mathrm{Ag} & 2.105374 & 1.411593 & 2.157026 \\ \mathrm{Ag} & 0.943866 & 1.410840 & -0.423554 \\ \mathrm{Ag} & 0.945061 & -1.410095 & -0.423556 \\ \mathrm{Ag} & 3.409438 & 2.787831 & -0.025656 \\ \mathrm{Ag} & 2.836960 & -1.456516 & -2.417354 \\ \mathrm{Ag} & -0.798010 & 2.319204 & -2.605250 \\ \mathrm{Ag} & 1.322301 & 3.832702 & -1.593888 \\ \mathrm{Ag} & 1.203869 & 3.866689 & 1.212787 \\ \mathrm{Ag} & 2.835807 & 1.458945 & -2.417299 \\ \mathrm{Ag} & 0.530061 & 0.000355 & -2.930480 \\ \mathrm{Ag} & 3.373686 & 0.001465 & -0.020704 \\ \mathrm{Ag} & -0.795928 & -2.319712 & -2.605305 \\ \mathrm{Ag} & -3.286866 & -2.112877 & -1.498682 \\ \mathrm{Ag} & -1.563558 & -0.000663 & -0.822629 \\ \mathrm{Ag} & -3.288666 & 2.110060 & -1.498518 \\ \mathrm{Ag} & -1.156662 & -3.113279 & 0.045268 \\ \mathrm{Ag} & -4.518950 & -0.001917 & -0.377456\end{array}$




$\begin{array}{lrrc}\mathrm{Ag} & -0.328667 & -0.000076 & 1.572607 \\ \mathrm{Ag} & -1.159393 & 3.112288 & 0.045411 \\ \mathrm{Ag} & -0.551315 & -2.368755 & 2.830775 \\ \mathrm{Ag} & -2.853974 & 1.403511 & 1.361115 \\ \mathrm{Ag} & -2.077281 & -0.000944 & 3.689593 \\ \mathrm{Ag} & -0.553435 & 2.368188 & 2.830855 \\ \mathrm{Ag} & 0.644229 & 0.000203 & 4.131986 \\ \mathrm{Ag} & -2.852683 & -1.405975 & 1.361059 \\ \mathrm{Ag} & -2.416560 & -0.000975 & -3.328200\end{array}$

$\begin{array}{lrrr}\mathrm{Ag} 31 & & & \\ \mathrm{E}=-4520.09067244 & \text { Hartree } & \\ \mathrm{Ag} & 0.482381 & 1.384984 & -0.846953 \\ \mathrm{Ag} & -0.622616 & 4.066874 & -0.748263 \\ \mathrm{Ag} & 2.684176 & 1.721569 & -2.740871 \\ \mathrm{Ag} & -0.049672 & 2.507451 & -3.174144 \\ \mathrm{Ag} & 1.993766 & 3.837032 & -1.244476 \\ \mathrm{Ag} & 3.108083 & 1.617442 & 0.088590 \\ \mathrm{Ag} & -1.515592 & 2.395338 & 1.325364 \\ \mathrm{Ag} & 1.091096 & -0.381754 & -3.369015 \\ \mathrm{Ag} & -1.825091 & -0.000951 & -0.000338 \\ \mathrm{Ag} & -2.468241 & 2.240047 & -1.498316 \\ \mathrm{Ag} & -0.618638 & -2.681853 & -3.148229 \\ \mathrm{Ag} & -1.513284 & -0.050697 & -2.738234 \\ \mathrm{Ag} & -4.102964 & 1.451285 & 0.710443 \\ \mathrm{Ag} & -4.101562 & -0.112514 & -1.615370 \\ \mathrm{Ag} & -2.466069 & -2.418873 & -1.192665 \\ \mathrm{Ag} & 1.088165 & 3.109550 & 1.353918 \\ \mathrm{Ag} & -0.050793 & 1.495235 & 3.758419 \\ \mathrm{Ag} & 2.683036 & 1.515331 & 2.861163 \\ \mathrm{Ag} & 0.482624 & 0.041536 & 1.622784 \\ \mathrm{Ag} & -0.620949 & -1.385805 & 3.896117 \\ \mathrm{Ag} & 3.109189 & -0.882621 & 1.356279 \\ \mathrm{Ag} & 0.483981 & -1.425559 & -0.775436 \\ \mathrm{Ag} & 1.994956 & -0.838956 & 3.944969 \\ \mathrm{Ag} & 1.090951 & -2.726285 & 2.015824 \\ \mathrm{Ag} & -2.468327 & 0.175228 & 2.689323 \\ \mathrm{Ag} & 3.109827 & -0.730402 & -1.442824 \\ \mathrm{Ag} & -1.513333 & -2.346923 & 1.411947 \\ \mathrm{Ag} & -4.101663 & -1.344817 & 0.901874 \\ \mathrm{Ag} & 1.997548 & -2.995296 & -2.699165 \\ \mathrm{Ag} & 2.686044 & -3.233027 & -0.118393 \\ \mathrm{Ag} & -0.047031 & -4.002569 & -0.584321\end{array}$

$\operatorname{Ag} 32$

$E=-4665.92831156$ Hartree 


$\begin{array}{lrrr}\mathrm{Ag} & -1.455701 & -2.366311 & 1.663912 \\ \mathrm{Ag} & 0.000013 & -0.000026 & -3.131168 \\ \mathrm{Ag} & -0.000006 & -1.426459 & -0.682058 \\ \mathrm{Ag} & -1.452095 & -3.739669 & -0.815142 \\ \mathrm{Ag} & -4.604538 & 0.000050 & -1.358896 \\ \mathrm{Ag} & -2.337557 & 1.444132 & -2.283799 \\ \mathrm{Ag} & -4.664037 & -0.000054 & 1.431208 \\ \mathrm{Ag} & -2.264116 & 0.000031 & 0.162749 \\ \mathrm{Ag} & 0.000020 & 2.856202 & -3.067133 \\ \mathrm{Ag} & -3.752499 & 2.311318 & 0.026813 \\ \mathrm{Ag} & -3.752537 & -2.311212 & 0.026688 \\ \mathrm{Ag} & -1.452060 & 3.739729 & -0.815128 \\ \mathrm{Ag} & -1.455394 & 2.366443 & 1.663924 \\ \mathrm{Ag} & -2.370974 & 0.000107 & 3.060886 \\ \mathrm{Ag} & -2.337530 & -1.444093 & -2.283868 \\ \mathrm{Ag} & 2.264119 & -0.000016 & 0.162819 \\ \mathrm{Ag} & 3.752476 & -2.311251 & 0.026877 \\ \mathrm{Ag} & 4.604553 & -0.000066 & -1.358889 \\ \mathrm{Ag} & 1.452003 & -3.739735 & -0.815170 \\ \mathrm{Ag} & 3.752583 & 2.311301 & 0.026710 \\ \mathrm{Ag} & 2.337556 & 1.444146 & -2.283804 \\ \mathrm{Ag} & 0.000026 & -2.856197 & -3.067224 \\ \mathrm{Ag} & 2.337595 & -1.444153 & -2.283808 \\ \mathrm{Ag} & -0.000033 & 1.426545 & -0.682123 \\ \mathrm{Ag} & 1.452100 & 3.739721 & -0.815102 \\ \mathrm{Ag} & 0.000001 & -0.000052 & 1.701010 \\ \mathrm{Ag} & 0.000012 & 1.420340 & 3.984914 \\ \mathrm{Ag} & 1.455665 & 2.366291 & 1.663920 \\ \mathrm{Ag} & 4.664062 & -0.000042 & 1.431188 \\ \mathrm{Ag} & 1.455435 & -2.366498 & 1.663855 \\ \mathrm{Ag} & -0.000072 & -1.420387 & 3.984890 \\ \mathrm{Ag} & 2.370928 & -0.000134 & 3.060950\end{array}$

Ag33
$\begin{array}{cccc}\mathrm{E}=-4811.74819247 \text { Hartree } & \\ \mathrm{Ag} & 1.387096 & 2.130555 & 2.057353 \\ \mathrm{Ag} & 2.329536 & 3.093207 & -0.634833 \\ \mathrm{Ag} & 2.249317 & 0.289347 & 0.030962 \\ \mathrm{Ag} & 4.103758 & 1.952880 & 1.244392 \\ \mathrm{Ag} & 1.405101 & -3.435648 & -1.631213 \\ \mathrm{Ag} & 3.318584 & -1.365347 & -1.851191 \\ \mathrm{Ag} & 0.000178 & -3.416676 & 0.855612 \\ \mathrm{Ag} & 2.797154 & -0.177055 & 2.684579 \\ \mathrm{Ag} & -0.000009 & -0.672130 & 1.436270 \\ \mathrm{Ag} & 1.337510 & -2.396419 & 3.261768 \\ \mathrm{Ag} & -1.337456 & -2.396622 & 3.261646\end{array}$




$\begin{array}{lrrr}\mathrm{Ag} & -0.000150 & 0.211336 & 3.898116 \\ \mathrm{Ag} & 4.277528 & 1.266269 & -1.525156 \\ \mathrm{Ag} & 2.528605 & -2.341419 & 0.730404 \\ \mathrm{Ag} & 1.348588 & -1.563829 & -3.726995 \\ \mathrm{Ag} & 4.776616 & -0.650526 & 0.532693 \\ \mathrm{Ag} & 0.000031 & -1.077535 & -1.293387 \\ \mathrm{Ag} & -1.404541 & -3.435734 & -1.631503 \\ \mathrm{Ag} & -2.797250 & -0.177399 & 2.684481 \\ \mathrm{Ag} & -1.387441 & 2.130358 & 2.057337 \\ \mathrm{Ag} & -0.000096 & 1.637250 & -0.327728 \\ \mathrm{Ag} & -2.249384 & 0.289252 & 0.031052 \\ \mathrm{Ag} & -1.541059 & 1.015575 & -2.596674 \\ \mathrm{Ag} & -0.000197 & 4.200532 & 0.553015 \\ \mathrm{Ag} & 1.540942 & 1.015660 & -2.596629 \\ \mathrm{Ag} & -0.000193 & 3.484674 & -2.272813 \\ \mathrm{Ag} & -1.348166 & -1.563870 & -3.727170 \\ \mathrm{Ag} & -2.329833 & 3.093034 & -0.634791 \\ \mathrm{Ag} & -4.277686 & 1.265975 & -1.525217 \\ \mathrm{Ag} & -4.104046 & 1.952496 & 1.244441 \\ \mathrm{Ag} & -3.318223 & -1.365519 & -1.851392 \\ \mathrm{Ag} & -4.776517 & -0.651053 & 0.532478 \\ \mathrm{Ag} & -2.528294 & -2.341618 & 0.730093\end{array}$

Ag34

$\mathrm{E}=-4957.57530995$ Hartree

$\begin{array}{llll}\mathrm{Ag} & 1.061732 & -3.159089 & -0.359831\end{array}$

$\begin{array}{llll}\mathrm{Ag} & 3.038689 & -1.342686 & 0.503818\end{array}$

$\begin{array}{lllll}\mathrm{Ag} & 3.017455 & -2.275737 & -2.176881\end{array}$

$\begin{array}{lllll}\mathrm{Ag} & 0.969984 & -0.491433 & -1.310488\end{array}$

$\begin{array}{llll}\mathrm{Ag} & 3.038705 & 1.342631 & -0.503613\end{array}$

$\begin{array}{llll}\text { Ag } & -0.721747 & -4.120986 & 1.520181\end{array}$

$\begin{array}{llll}\mathrm{Ag} & -1.571264 & -3.609751 & -1.184034\end{array}$

$\begin{array}{llll}\mathrm{Ag} & 2.974626 & 0.302547 & -3.142104\end{array}$

$\begin{array}{llll}\mathrm{Ag} & 0.991431 & -2.133812 & 2.308103\end{array}$

$\begin{array}{llll}\mathrm{Ag} & 4.960187 & 0.477079 & 1.322078\end{array}$

$\begin{array}{llll}\text { Ag } & 0.969927 & 0.491416 & 1.310512\end{array}$

$\begin{array}{llll}\mathrm{Ag} & 3.017378 & 2.275646 & 2.177094\end{array}$

$\begin{array}{llll}\mathrm{Ag} & 2.974370 & -0.302736 & 3.142324\end{array}$

$\begin{array}{llll}\mathrm{Ag} & 0.381501 & -2.628130 & -3.041142\end{array}$

$\begin{array}{llll}\mathrm{Ag} & 4.960260 & -0.477296 & -1.321804\end{array}$

$\begin{array}{llll}\mathrm{Ag} & 0.317309 & 0.033363 & -4.013018\end{array}$

$\begin{array}{llll}\mathrm{Ag} & -1.665402 & -0.884617 & -2.297211\end{array}$

$\begin{array}{llll}\mathrm{Ag} & -1.143604 & 1.393631 & -0.430498\end{array}$

$\begin{array}{llll}\mathrm{Ag} & -1.143875 & -1.393584 & 0.430395\end{array}$

$\begin{array}{llll}\text { Ag } & -3.884714 & 2.089137 & 1.147158\end{array}$

$\begin{array}{llll}\text { Ag } & 0.991755 & 2.133859 & -2.308078\end{array}$ 


$\begin{array}{lrrr}\mathrm{Ag} & -1.729824 & 1.864724 & -3.067304 \\ \mathrm{Ag} & 1.061854 & 3.159138 & 0.359846 \\ \mathrm{Ag} & -0.721522 & 4.120971 & -1.520252 \\ \mathrm{Ag} & -3.259971 & 3.367125 & -1.173659 \\ \mathrm{Ag} & -1.665532 & 0.884652 & 2.297036 \\ \mathrm{Ag} & -3.735870 & 0.665529 & -1.260620 \\ \mathrm{Ag} & -3.736069 & -0.665454 & 1.260618 \\ \mathrm{Ag} & -3.884691 & -2.088972 & -1.147276 \\ \mathrm{Ag} & -1.730103 & -1.864661 & 3.067130 \\ \mathrm{Ag} & -3.260209 & -3.367062 & 1.173500 \\ \mathrm{Ag} & 0.381381 & 2.628096 & 3.041079 \\ \mathrm{Ag} & 0.317081 & -0.033311 & 4.013030 \\ \mathrm{Ag} & -1.571227 & 3.609769 & 1.183911\end{array}$

$\begin{array}{lrrc}\mathrm{Ag} 35 & & & \\ \mathrm{E}=-5103.38912758 & \text { Hartree } & \\ \mathrm{Ag} & -2.033629 & 1.787139 & 2.171221 \\ \mathrm{Ag} & -2.270104 & 0.000021 & -0.000059 \\ \mathrm{Ag} & 0.000090 & -1.378478 & -0.954102 \\ \mathrm{Ag} & -2.033259 & -2.773965 & 0.462090 \\ \mathrm{Ag} & -4.584951 & 0.616040 & -1.492573 \\ \mathrm{Ag} & -4.585059 & 0.984261 & 1.279780 \\ \mathrm{Ag} & 1.254544 & -3.220910 & -2.790581 \\ \mathrm{Ag} & -1.254329 & -3.746001 & -2.032127 \\ \mathrm{Ag} & -4.584786 & -1.600819 & 0.212505 \\ \mathrm{Ag} & 0.576949 & -3.807836 & 0.146063 \\ \mathrm{Ag} & -2.960004 & -1.012837 & 2.470208 \\ \mathrm{Ag} & 2.033304 & -2.117215 & 1.850811 \\ \mathrm{Ag} & -1.254551 & 0.113217 & 4.260125 \\ \mathrm{Ag} & 2.960000 & -2.551874 & -0.784489 \\ \mathrm{Ag} & 1.254405 & -0.806148 & 4.184705 \\ \mathrm{Ag} & 0.576696 & 2.030547 & 3.224374 \\ \mathrm{Ag} & -0.576846 & -2.528945 & 2.850500 \\ \mathrm{Ag} & 4.584855 & -1.179944 & 1.102424 \\ \mathrm{Ag} & 2.269995 & 0.000094 & 0.000063 \\ \mathrm{Ag} & -2.959837 & -1.632848 & -2.112349 \\ \mathrm{Ag} & -0.576635 & -1.203836 & -3.615258 \\ \mathrm{Ag} & -0.000076 & -0.137159 & 1.670812 \\ \mathrm{Ag} & 2.033450 & 2.661628 & 0.908119 \\ \mathrm{Ag} & -2.033500 & 0.986825 & -2.633373 \\ \mathrm{Ag} & -1.254495 & 3.632803 & -2.228050 \\ \mathrm{Ag} & -2.960062 & 2.645660 & -0.357978 \\ \mathrm{Ag} & 2.960062 & 1.955419 & -1.817680 \\ \mathrm{Ag} & 2.959829 & 0.596620 & 2.602318 \\ \mathrm{Ag} & 2.033656 & -0.544233 & -2.759001 \\ \mathrm{Ag} & 4.584945 & 1.544604 & 0.470840\end{array}$




$$
\begin{array}{lrrr}
\mathrm{Ag} & 4.585014 & -0.364621 & -1.572785 \\
\mathrm{Ag} & 0.576764 & 1.777306 & -3.370571 \\
\mathrm{Ag} & 1.254400 & 4.027092 & -1.394246 \\
\mathrm{Ag} & -0.576806 & 3.732919 & 0.764957 \\
\mathrm{Ag} & -0.000030 & 1.515472 & -0.716693
\end{array}
$$

$\begin{array}{lrcc}\mathrm{Ag} 36 & & & \\ \mathrm{E}=-5249.19295390 & \text { Hartree } & \\ \mathrm{Ag} & 4.637232 & -1.956428 & -0.973693 \\ \mathrm{Ag} & 3.792822 & 0.740069 & -1.294812 \\ \mathrm{Ag} & 2.365659 & 0.636218 & 2.606301 \\ \mathrm{Ag} & -3.837402 & -2.916321 & 1.524514 \\ \mathrm{Ag} & 2.281305 & -1.098846 & 0.251321 \\ \mathrm{Ag} & 3.837607 & -2.916177 & 1.524584 \\ \mathrm{Ag} & 2.373234 & -3.637448 & -0.819184 \\ \mathrm{Ag} & 4.686123 & -0.201499 & 1.239609 \\ \mathrm{Ag} & -0.000116 & -2.909426 & 0.525087 \\ \mathrm{Ag} & -1.495683 & -2.115758 & 2.865738 \\ \mathrm{Ag} & 1.495590 & -2.115882 & 2.865654 \\ \mathrm{Ag} & -0.000098 & -0.837330 & -4.046928 \\ \mathrm{Ag} & -2.281272 & -1.098888 & 0.251313 \\ \mathrm{Ag} & -2.373329 & -3.637493 & -0.819344 \\ \mathrm{Ag} & 2.355135 & -1.414398 & -2.580052 \\ \mathrm{Ag} & -0.000002 & -3.112741 & -2.317557 \\ \mathrm{Ag} & -4.637336 & -1.956288 & -0.973639 \\ \mathrm{Ag} & -2.355225 & -1.414414 & -2.580006 \\ \mathrm{Ag} & -3.792960 & 0.740078 & -1.294694 \\ \mathrm{Ag} & -1.437335 & 1.314016 & -2.834642 \\ \mathrm{Ag} & 3.780704 & 2.475446 & 0.986509 \\ \mathrm{Ag} & 1.404372 & 1.558550 & -0.007961 \\ \mathrm{Ag} & 1.419543 & 4.165135 & 0.820649 \\ \mathrm{Ag} & -0.000033 & -0.568220 & -1.291321 \\ \mathrm{Ag} & -1.404405 & 1.558643 & -0.007924 \\ \mathrm{Ag} & -0.000040 & 3.468973 & -1.561512 \\ \mathrm{Ag} & 2.839987 & 3.412130 & -1.500848 \\ \mathrm{Ag} & 0.000160 & 2.328744 & 2.437573 \\ \mathrm{Ag} & 1.437225 & 1.313960 & -2.834783 \\ \mathrm{Ag} & -2.840039 & 3.412177 & -1.500713 \\ \mathrm{Ag} & -1.419485 & 4.165017 & 0.820774 \\ \mathrm{Ag} & -4.685929 & -0.201538 & 1.239852 \\ \mathrm{Ag} & -3.780747 & 2.475519 & 0.986719 \\ \mathrm{Ag} & 0.000010 & -0.280450 & 1.510334 \\ \mathrm{Ag} & -2.365403 & 0.636364 & 2.606440 \\ \mathrm{Ag} & 0.000131 & -0.011495 & 4.176644\end{array}$

Ag37 


$\begin{array}{lrrr}\mathrm{E}=-5395.01137973 & \text { Hartree } & \\ \mathrm{Ag} & 0.984780 & 1.901553 & -2.833277 \\ \mathrm{Ag} & 2.137112 & 3.979631 & -1.311675 \\ \mathrm{Ag} & 3.610103 & 1.485016 & -1.577806 \\ \mathrm{Ag} & 1.387299 & 1.604060 & -0.076315 \\ \mathrm{Ag} & 4.095971 & 0.528551 & 1.078056 \\ \mathrm{Ag} & -0.624242 & 3.797308 & -1.291159 \\ \mathrm{Ag} & 2.230345 & -1.049878 & -0.066890 \\ \mathrm{Ag} & -2.137481 & 3.979428 & 1.312919 \\ \mathrm{Ag} & 3.422119 & 3.193583 & 0.959741 \\ \mathrm{Ag} & -0.985081 & 1.900672 & 2.834102 \\ \mathrm{Ag} & 1.868060 & 1.542566 & 2.679382 \\ \mathrm{Ag} & 0.623800 & 3.796041 & 1.291274 \\ \mathrm{Ag} & 2.257181 & -0.730932 & -2.896515 \\ \mathrm{Ag} & 4.617623 & -1.112832 & -1.445542 \\ \mathrm{Ag} & 2.453380 & -1.239014 & 2.748845 \\ \mathrm{Ag} & 4.494480 & -2.279365 & 1.011924 \\ \mathrm{Ag} & 0.088340 & -0.329893 & 1.477022 \\ \mathrm{Ag} & -3.422121 & 3.193543 & -0.958639 \\ \mathrm{Ag} & 0.124709 & -2.709728 & -2.823252 \\ \mathrm{Ag} & -0.088349 & -0.329674 & -1.476922 \\ \mathrm{Ag} & 2.607659 & -3.269919 & -1.460257 \\ \mathrm{Ag} & -0.235496 & -0.255025 & -4.226032 \\ \mathrm{Ag} & -1.868095 & 1.543174 & -2.678855 \\ \mathrm{Ag} & -4.494372 & -2.279046 & -1.012077 \\ \mathrm{Ag} & -2.453477 & -1.238269 & -2.749092 \\ \mathrm{Ag} & -1.385884 & 1.604956 & 0.076642 \\ \mathrm{Ag} & -2.257091 & -0.732020 & 2.896771 \\ \mathrm{Ag} & -2.230585 & -1.048888 & 0.066892 \\ \mathrm{Ag} & -2.179834 & -3.647170 & -1.371656 \\ \mathrm{Ag} & -2.607687 & -3.269941 & 1.459015 \\ \mathrm{Ag} & -0.000244 & -2.813581 & -0.000315 \\ \mathrm{Ag} & -4.096516 & 0.528795 & -1.078557 \\ \mathrm{Ag} & -0.124577 & -2.710434 & 2.822630 \\ \mathrm{Ag} & 0.235541 & -0.256223 & 4.226154 \\ \mathrm{Ag} & 2.179733 & -3.647609 & 1.370455 \\ \mathrm{Ag} & -4.617804 & -1.113402 & 1.445666 \\ \mathrm{Ag} & -3.609299 & 1.483964 & 1.577345\end{array}$

Ag38

$E=-5540.83025399$ Hartree

$\begin{array}{rrrr}\mathrm{Ag} & 1.131722 & 4.274685 & 0.561109 \\ \mathrm{Ag} & 0.885417 & 1.718632 & -0.562799 \\ \mathrm{Ag} & -1.458706 & 3.210613 & 0.212262 \\ \mathrm{Ag} & 2.376602 & 2.535544 & -2.791327 \\ \mathrm{Ag} & -1.694992 & 0.570255 & -0.925169\end{array}$




$\begin{array}{rrrr}\mathrm{Ag} & -2.922623 & 2.307765 & 2.449660 \\ \mathrm{Ag} & -2.484066 & 2.826870 & -2.388846 \\ \mathrm{Ag} & -0.630322 & 0.880624 & 1.697482 \\ \mathrm{Ag} & 0.080243 & 3.968198 & -2.028698 \\ \mathrm{Ag} & -0.181669 & 1.426592 & -3.226895 \\ \mathrm{Ag} & -2.735893 & 0.260177 & -3.509364 \\ \mathrm{Ag} & -4.232636 & -0.567258 & -1.277375 \\ \mathrm{Ag} & -3.252399 & -0.271291 & 1.352512 \\ \mathrm{Ag} & -3.980777 & 1.999398 & -0.156639 \\ \mathrm{Ag} & -2.123127 & 0.047428 & 3.919087 \\ \mathrm{Ag} & 0.424990 & 1.181648 & 4.276925 \\ \mathrm{Ag} & 1.975453 & 2.055351 & 2.086640 \\ \mathrm{Ag} & -0.374470 & 3.441867 & 2.807427 \\ \mathrm{Ag} & 3.428019 & 2.841934 & -0.201390 \\ \mathrm{Ag} & 3.252397 & 0.271297 & -1.352509 \\ \mathrm{Ag} & 0.630320 & -0.880618 & -1.697479 \\ \mathrm{Ag} & 2.123159 & -0.047513 & -3.919058 \\ \mathrm{Ag} & -1.975455 & -2.055345 & -2.086637 \\ \mathrm{Ag} & -3.428021 & -2.841927 & 0.201394 \\ \mathrm{Ag} & -0.424992 & -1.181642 & -4.276921 \\ \mathrm{Ag} & 0.374468 & -3.441861 & -2.807424 \\ \mathrm{Ag} & -0.885419 & -1.718626 & 0.562803 \\ \mathrm{Ag} & 1.694990 & -0.570249 & 0.925173 \\ \mathrm{Ag} & 2.922621 & -2.307759 & -2.449657 \\ \mathrm{Ag} & 0.181667 & -1.426586 & 3.226898 \\ \mathrm{Ag} & 1.458704 & -3.210607 & -0.212258 \\ \mathrm{Ag} & 2.484064 & -2.826864 & 2.388850 \\ \mathrm{Ag} & -1.131722 & -4.274705 & -0.561202 \\ \mathrm{Ag} & -0.080245 & -3.968192 & 2.028702 \\ \mathrm{Ag} & 3.980776 & -1.999391 & 0.156642 \\ \mathrm{Ag} & 4.232634 & 0.567264 & 1.277379 \\ \mathrm{Ag} & 2.735891 & -0.260171 & 3.509368 \\ \mathrm{Ag} & -2.376604 & -2.535538 & 2.791331 \\ & & & \\ \end{array}$

Ag39

$E=-5686.65586010$ Hartree

$\begin{array}{lrrr}\mathrm{Ag} & 1.020636 & -2.174248 & -0.115643 \\ \mathrm{Ag} & -1.752484 & -1.642569 & -0.115661 \\ \mathrm{Ag} & -2.238995 & -4.105379 & -1.195009 \\ \mathrm{Ag} & -1.801467 & -1.688501 & 2.731023 \\ \mathrm{Ag} & -0.751880 & -3.920763 & 1.238439 \\ \mathrm{Ag} & -3.541799 & -3.319480 & 1.180213 \\ \mathrm{Ag} & -3.961250 & -0.496541 & 1.238372 \\ \mathrm{Ag} & -3.182860 & 3.425653 & -1.195087 \\ \mathrm{Ag} & -4.241517 & -1.968560 & -1.195109 \\ \mathrm{Ag} & -1.057959 & 2.254264 & -2.634101\end{array}$




$\begin{array}{lrrr}\mathrm{Ag} & -4.251527 & 2.342647 & 1.180254 \\ \mathrm{Ag} & -1.696306 & 3.613847 & 1.238453 \\ \mathrm{Ag} & -2.162492 & 1.191548 & 2.731071 \\ \mathrm{Ag} & -4.596372 & 0.860742 & -1.195021 \\ \mathrm{Ag} & 0.000047 & 0.000042 & -4.142424 \\ \mathrm{Ag} & -2.103733 & 1.159116 & -0.115616 \\ \mathrm{Ag} & -0.469103 & -2.445517 & -2.634171 \\ \mathrm{Ag} & -2.470764 & -0.309599 & -2.634146 \\ \mathrm{Ag} & 0.000036 & -0.000008 & -1.462187 \\ \mathrm{Ag} & 0.561486 & -4.642290 & -1.195112 \\ \mathrm{Ag} & 3.496548 & -1.926596 & 1.238408 \\ \mathrm{Ag} & 2.383266 & 0.298751 & -0.115629 \\ \mathrm{Ag} & 0.452288 & 2.358884 & -0.115603 \\ \mathrm{Ag} & 4.588551 & -0.900536 & -1.195078 \\ \mathrm{Ag} & 3.212611 & -3.397974 & -1.194937 \\ \mathrm{Ag} & 2.912815 & 2.730019 & 1.238432 \\ \mathrm{Ag} & 2.180958 & -1.201810 & -2.634186 \\ \mathrm{Ag} & 1.816929 & 1.702816 & -2.634183 \\ \mathrm{Ag} & 2.274408 & 4.085739 & -1.195069 \\ \mathrm{Ag} & 4.224462 & 2.005285 & -1.194991 \\ \mathrm{Ag} & 4.816504 & 0.603721 & 1.180260 \\ \mathrm{Ag} & 2.449827 & 0.307075 & 2.731105 \\ \mathrm{Ag} & 0.000039 & 0.000002 & 1.395940 \\ \mathrm{Ag} & 0.914172 & 4.767416 & 1.180253 \\ \mathrm{Ag} & -0.000012 & -0.000086 & 4.159370 \\ \mathrm{Ag} & 1.049087 & -2.235033 & 2.731067 \\ \mathrm{Ag} & 2.062617 & -4.394245 & 1.180219 \\ \mathrm{Ag} & -0.601769 & 4.637318 & -1.194929 \\ \mathrm{Ag} & 0.465001 & 2.424848 & 2.731013\end{array}$

Ag40

$\mathrm{E}=-5832.45728180$ Hartree

$\begin{array}{lccc}\mathrm{Ag} & 0.000262 & -2.356066 & 0.067725 \\ \mathrm{Ag} & 2.425442 & -1.693853 & -2.582661 \\ \mathrm{Ag} & 4.115798 & -2.837140 & -0.740000 \\ \mathrm{Ag} & -1.667401 & -4.149859 & -1.077518 \\ \mathrm{Ag} & 4.559892 & -0.004331 & -1.184872 \\ \mathrm{Ag} & -2.370052 & -3.160046 & 1.499769 \\ \mathrm{Ag} & 1.668373 & -4.149508 & -1.077521 \\ \mathrm{Ag} & -0.000076 & -0.189816 & -4.127774 \\ \mathrm{Ag} & 2.370817 & -3.159493 & 1.499780 \\ \mathrm{Ag} & 0.000545 & -4.777090 & 1.223659 \\ \mathrm{Ag} & 2.349267 & 1.014568 & -2.675086 \\ \mathrm{Ag} & -4.115140 & -2.838018 & -0.740076 \\ \mathrm{Ag} & -2.424916 & -1.694320 & -2.582690 \\ \mathrm{Ag} & -4.559917 & -0.005357 & -1.184911\end{array}$




$\begin{array}{lrrr}\mathrm{Ag} & -2.349588 & 1.014120 & -2.675101 \\ \mathrm{Ag} & 0.000064 & -0.167096 & -1.515491 \\ \mathrm{Ag} & 0.000331 & -2.802059 & -2.875687 \\ \mathrm{Ag} & -2.288856 & -0.777511 & 0.035064 \\ \mathrm{Ag} & -4.651974 & -1.313442 & 1.447845 \\ \mathrm{Ag} & 0.000250 & -2.366318 & 2.886329 \\ \mathrm{Ag} & 1.409696 & 1.907879 & -0.153505 \\ \mathrm{Ag} & -1.440131 & 2.115221 & 2.723280 \\ \mathrm{Ag} & 1.439689 & 2.115547 & 2.723220 \\ \mathrm{Ag} & 3.854796 & 2.784400 & -1.155801 \\ \mathrm{Ag} & 4.652238 & -1.312383 & 1.447800 \\ \mathrm{Ag} & 3.773048 & 1.324411 & 1.268863 \\ \mathrm{Ag} & 2.330725 & -0.628658 & 2.872369 \\ \mathrm{Ag} & -0.000425 & 4.018781 & 1.124691 \\ \mathrm{Ag} & 2.289057 & -0.777018 & 0.035098 \\ \mathrm{Ag} & -2.330569 & -0.629184 & 2.872376 \\ \mathrm{Ag} & -0.000051 & 0.171607 & 4.238204 \\ \mathrm{Ag} & -0.000266 & 2.442396 & -2.681996 \\ \mathrm{Ag} & 1.442823 & 4.399431 & -1.301354 \\ \mathrm{Ag} & -0.000060 & 0.072168 & 1.456590 \\ \mathrm{Ag} & -1.443825 & 4.399142 & -1.301383 \\ \mathrm{Ag} & -2.819016 & 3.996823 & 1.125593 \\ \mathrm{Ag} & -3.773340 & 1.323499 & 1.268916 \\ \mathrm{Ag} & -3.855464 & 2.783563 & -1.155744 \\ \mathrm{Ag} & -1.410178 & 1.907591 & -0.153520 \\ \mathrm{Ag} & 2.818132 & 3.997420 & 1.125524\end{array}$

Ag41

$E=-5978.29039804$ Hartree

$\begin{array}{lrrr}\mathrm{Ag} & -1.705773 & 1.453422 & -2.826258 \\ \mathrm{Ag} & 0.015381 & 4.706819 & -1.014731 \\ \mathrm{Ag} & -0.898172 & 2.284978 & -0.111989 \\ \mathrm{Ag} & 0.781204 & 3.794350 & 1.588703 \\ \mathrm{Ag} & -1.900891 & 4.611223 & 1.035314 \\ \mathrm{Ag} & 1.771451 & 1.431645 & 0.333644 \\ \mathrm{Ag} & 3.450161 & 2.860753 & 2.020339 \\ \mathrm{Ag} & -2.695486 & 3.764926 & -1.542280 \\ \mathrm{Ag} & 0.083927 & 0.000032 & -1.312163 \\ \mathrm{Ag} & 1.000565 & 2.409389 & -2.330420 \\ \mathrm{Ag} & 2.701619 & 3.877571 & -0.514105 \\ \mathrm{Ag} & 1.278889 & 1.453173 & 3.168148 \\ \mathrm{Ag} & 1.771240 & -1.431969 & 0.333653 \\ \mathrm{Ag} & 3.743081 & 2.496649 & -2.639625 \\ \mathrm{Ag} & 2.761247 & -0.000219 & -2.016924 \\ \mathrm{Ag} & 4.469490 & 1.439599 & -0.201872 \\ \mathrm{Ag} & 4.469275 & -1.440283 & -0.201973\end{array}$




$\begin{array}{lrrr}\mathrm{Ag} & 3.496561 & -0.000299 & 2.074109 \\ \mathrm{Ag} & 3.742553 & -2.497193 & -2.639736 \\ \mathrm{Ag} & 0.581359 & -0.000102 & -3.941212 \\ \mathrm{Ag} & 2.700990 & -3.877915 & -0.514186 \\ \mathrm{Ag} & 1.278675 & -1.453364 & 3.168131 \\ \mathrm{Ag} & 3.449806 & -2.861264 & 2.020253 \\ \mathrm{Ag} & -0.936078 & 0.000114 & 4.216474 \\ \mathrm{Ag} & -1.442193 & 2.339982 & 2.681352 \\ \mathrm{Ag} & -0.419184 & 0.000100 & 1.490849 \\ \mathrm{Ag} & -4.348600 & 1.461084 & -1.846582 \\ \mathrm{Ag} & -3.596258 & 2.343178 & 0.763565 \\ \mathrm{Ag} & -3.102692 & 0.000289 & 2.364120 \\ \mathrm{Ag} & -3.596696 & -2.342566 & 0.763741 \\ \mathrm{Ag} & -2.527839 & 0.000191 & -0.418732 \\ \mathrm{Ag} & -1.901565 & -4.610914 & 1.035402 \\ \mathrm{Ag} & -1.706102 & -1.453209 & -2.826186 \\ \mathrm{Ag} & -2.696127 & -3.764505 & -1.542246 \\ \mathrm{Ag} & -5.179271 & 0.000355 & 0.416758 \\ \mathrm{Ag} & -4.348890 & -1.460501 & -1.846515 \\ \mathrm{Ag} & -0.898590 & -2.284858 & -0.111916 \\ \mathrm{Ag} & -1.442557 & -2.339714 & 2.681425 \\ \mathrm{Ag} & 0.780700 & -3.794489 & 1.588779 \\ \mathrm{Ag} & 1.000130 & -2.409582 & -2.330434 \\ \mathrm{Ag} & 0.014660 & -4.706875 & -1.014673\end{array}$

Ag42

$E=-6124.11889389$ Hartree

$\begin{array}{lrrr}\mathrm{Ag} & -1.586560 & 1.461125 & -2.920446 \\ \mathrm{Ag} & -1.023794 & 2.286530 & -0.149347 \\ \mathrm{Ag} & -2.110263 & 4.617294 & 0.895895 \\ \mathrm{Ag} & -4.306420 & 1.463843 & -2.181347 \\ \mathrm{Ag} & -2.685004 & 3.770277 & -1.732759 \\ \mathrm{Ag} & -1.586407 & -1.461131 & -2.920572 \\ \mathrm{Ag} & -4.306312 & -1.464049 & -2.181412 \\ \mathrm{Ag} & -5.339606 & -0.000234 & -0.002757 \\ \mathrm{Ag} & -2.684677 & -3.770325 & -1.732914 \\ \mathrm{Ag} & -3.788478 & -2.347868 & 0.484095 \\ \mathrm{Ag} & -2.630262 & -0.000109 & -0.593338 \\ \mathrm{Ag} & -3.449803 & -0.000167 & 2.124557 \\ \mathrm{Ag} & -3.788725 & 2.347564 & 0.484199 \\ \mathrm{Ag} & -0.702311 & -0.000075 & 1.499472 \\ \mathrm{Ag} & -2.109856 & -4.617390 & 0.895757 \\ \mathrm{Ag} & -1.463778 & -0.000092 & 4.162908 \\ \mathrm{Ag} & -1.815264 & -2.346788 & 2.582534 \\ \mathrm{Ag} & -1.815457 & 2.346566 & 2.582539 \\ \mathrm{Ag} & -0.007248 & -4.688374 & -0.977746\end{array}$




$\begin{array}{lrrr}\mathrm{Ag} & 0.836488 & -1.457802 & 3.326582 \\ \mathrm{Ag} & 0.509591 & -3.799015 & 1.683086 \\ \mathrm{Ag} & -1.023593 & -2.286567 & -0.149418 \\ \mathrm{Ag} & 0.836358 & 1.457833 & 3.326585 \\ \mathrm{Ag} & 0.509329 & 3.799003 & 1.683083 \\ \mathrm{Ag} & 2.634978 & 3.827460 & -0.257230 \\ \mathrm{Ag} & 0.035206 & 0.000021 & -1.246159 \\ \mathrm{Ag} & 1.099554 & 2.392391 & -2.189766 \\ \mathrm{Ag} & -0.007544 & 4.688366 & -0.977787 \\ \mathrm{Ag} & 1.585947 & 1.425851 & 0.558676 \\ \mathrm{Ag} & 3.935879 & -2.393078 & -2.277562 \\ \mathrm{Ag} & 3.935710 & 2.393307 & -2.277537 \\ \mathrm{Ag} & 0.764502 & 0.000080 & -3.832415 \\ \mathrm{Ag} & 1.586111 & -1.425704 & 0.558727 \\ \mathrm{Ag} & 3.143102 & 0.000122 & 2.457578 \\ \mathrm{Ag} & 2.724496 & 0.000025 & -1.687046 \\ \mathrm{Ag} & 4.352349 & -1.431870 & 0.278629 \\ \mathrm{Ag} & 3.106572 & 2.866075 & 2.378781 \\ \mathrm{Ag} & 5.441242 & 0.000163 & -1.886699 \\ \mathrm{Ag} & 4.352283 & 1.432089 & 0.278685 \\ \mathrm{Ag} & 3.106682 & -2.865841 & 2.378827 \\ \mathrm{Ag} & 1.099731 & -2.392269 & -2.189738 \\ \mathrm{Ag} & 2.635252 & -3.827235 & -0.257200\end{array}$

$\mathrm{Ag} 43$

$E=-6269.92305287$ Hartree

$\begin{array}{lrrr}\mathrm{Ag} & -2.653477 & 0.000210 & -0.784553 \\ \mathrm{Ag} & -4.136616 & 1.464997 & -2.549015 \\ \mathrm{Ag} & -1.351718 & 1.455716 & -2.992851 \\ \mathrm{Ag} & -4.136988 & -1.464264 & -2.548931 \\ \mathrm{Ag} & -3.764665 & 0.000341 & 1.828997 \\ \mathrm{Ag} & -0.973002 & 0.000076 & 1.511899 \\ \mathrm{Ag} & -2.178444 & 2.351500 & 2.460852 \\ \mathrm{Ag} & -5.406757 & 0.000489 & -0.496559 \\ \mathrm{Ag} & -2.572407 & 3.768026 & -1.930375 \\ \mathrm{Ag} & -1.104981 & 2.287584 & -0.170119 \\ \mathrm{Ag} & -3.917506 & 2.349833 & 0.159590 \\ \mathrm{Ag} & 0.372176 & 1.457271 & 3.501489 \\ \mathrm{Ag} & -2.010426 & 0.000086 & 4.072907 \\ \mathrm{Ag} & 1.229443 & 2.378825 & -1.975041 \\ \mathrm{Ag} & 2.736375 & 2.870295 & 2.786790 \\ \mathrm{Ag} & 4.051364 & 2.317627 & -1.875796 \\ \mathrm{Ag} & 0.013396 & 4.679842 & -0.884093 \\ \mathrm{Ag} & 2.571281 & 3.785712 & 0.085507 \\ \mathrm{Ag} & -2.287859 & 4.620179 & 0.745456 \\ \mathrm{Ag} & 1.386795 & 1.434536 & 0.849924\end{array}$




$\begin{array}{lrcc}\mathrm{Ag} & 0.231773 & 3.811173 & 1.817267 \\ \mathrm{Ag} & 4.202440 & 1.455241 & 0.819677 \\ \mathrm{Ag} & 5.543039 & -0.000386 & -1.224863 \\ \mathrm{Ag} & 2.770791 & -0.000331 & 2.855347 \\ \mathrm{Ag} & 0.371946 & -1.457569 & 3.501389 \\ \mathrm{Ag} & 2.763284 & -0.000162 & -1.145656 \\ \mathrm{Ag} & 1.189055 & -0.000124 & -3.616871 \\ \mathrm{Ag} & 4.050840 & -2.318039 & -1.876126 \\ \mathrm{Ag} & 4.000866 & 0.000051 & -3.553744 \\ \mathrm{Ag} & -1.105385 & -2.287438 & -0.170241 \\ \mathrm{Ag} & 1.386517 & -1.434746 & 0.849832 \\ \mathrm{Ag} & 0.058337 & -0.000035 & -1.143741 \\ \mathrm{Ag} & -1.352039 & -1.455455 & -2.992893 \\ \mathrm{Ag} & -2.288743 & -4.619922 & 0.745455 \\ \mathrm{Ag} & -3.917955 & -2.349195 & 0.159605 \\ \mathrm{Ag} & -2.178899 & -2.351217 & 2.460822 \\ \mathrm{Ag} & -2.573124 & -3.767567 & -1.930300 \\ \mathrm{Ag} & 2.735919 & -2.870914 & 2.786570 \\ \mathrm{Ag} & 0.012486 & -4.679808 & -0.884227 \\ \mathrm{Ag} & 1.228844 & -2.379025 & -1.975105 \\ \mathrm{Ag} & 0.231124 & -3.811305 & 1.817137 \\ \mathrm{Ag} & 4.202202 & -1.455983 & 0.819411 \\ \mathrm{Ag} & 2.570695 & -3.786127 & 0.085177\end{array}$

Ag44

$E=-6415.73765360$ Hartree

$\begin{array}{lrcc}\mathrm{Ag} & 4.196103 & 1.985700 & -0.317834 \\ \mathrm{Ag} & 1.371222 & 2.182760 & -0.418617 \\ \mathrm{Ag} & 2.849735 & 4.460153 & 0.147300 \\ \mathrm{Ag} & 2.816589 & 3.323496 & -2.434654 \\ \mathrm{Ag} & 1.294308 & 1.058097 & -3.142148 \\ \mathrm{Ag} & -1.093498 & 2.343699 & -2.026006 \\ \mathrm{Ag} & 0.432580 & 4.599746 & -1.295278 \\ \mathrm{Ag} & -0.088466 & -0.059129 & -1.051238 \\ \mathrm{Ag} & -4.265385 & 0.188083 & -3.161718 \\ \mathrm{Ag} & -3.188092 & -2.265093 & -3.693016 \\ \mathrm{Ag} & 4.092560 & 0.817259 & -2.922586 \\ \mathrm{Ag} & -1.421408 & -0.166541 & -3.438686 \\ \mathrm{Ag} & 0.942538 & -2.344282 & 0.085196 \\ \mathrm{Ag} & -1.525934 & -2.398673 & -1.497846 \\ \mathrm{Ag} & -0.447591 & -4.686977 & -0.254650 \\ \mathrm{Ag} & 2.095132 & -4.136492 & -1.613744 \\ \mathrm{Ag} & 3.754248 & -2.661941 & 0.193173 \\ \mathrm{Ag} & 1.984188 & -4.679662 & 1.158156 \\ \mathrm{Ag} & 2.634423 & -0.302740 & -0.901262 \\ \mathrm{Ag} & 3.969060 & -0.154078 & 1.602351\end{array}$




$\begin{array}{lrrr}\mathrm{Ag} & 1.011003 & -1.830287 & -2.833187 \\ \mathrm{Ag} & 5.391732 & -0.558526 & -0.830215 \\ \mathrm{Ag} & 3.810309 & -2.083221 & -2.604673 \\ \mathrm{Ag} & -2.100282 & 4.086194 & -0.028074 \\ \mathrm{Ag} & -3.884765 & 2.599486 & -1.679394 \\ \mathrm{Ag} & -5.535562 & 0.512392 & -0.684224 \\ \mathrm{Ag} & -1.363338 & -1.136622 & 1.189758 \\ \mathrm{Ag} & 2.235164 & -2.250073 & 2.613266 \\ \mathrm{Ag} & -0.350509 & -3.509159 & 2.325702 \\ \mathrm{Ag} & -3.875815 & 1.996566 & 1.070674 \\ \mathrm{Ag} & -4.153045 & -0.872898 & 1.380045 \\ \mathrm{Ag} & -2.824839 & -3.426868 & 0.793733 \\ \mathrm{Ag} & -4.363824 & -2.030318 & -1.210083 \\ \mathrm{Ag} & -2.778777 & 0.198681 & -0.872874 \\ \mathrm{Ag} & -2.418181 & 0.622314 & 3.136136 \\ \mathrm{Ag} & -0.120342 & -0.999884 & 3.742792 \\ \mathrm{Ag} & -2.662327 & -2.225076 & 3.375168 \\ \mathrm{Ag} & 0.152565 & 1.881176 & 3.420656 \\ \mathrm{Ag} & -1.098837 & 1.693780 & 0.868816 \\ \mathrm{Ag} & 1.171334 & 0.103193 & 1.504672 \\ \mathrm{Ag} & 2.429135 & 0.254739 & 3.963895 \\ \mathrm{Ag} & 2.677827 & 2.398572 & 2.098427 \\ \mathrm{Ag} & -2.119017 & 3.449679 & 2.750690 \\ \mathrm{Ag} & 0.368079 & 4.022774 & 1.491402\end{array}$

Ag45

$E=-6561.55991700$ Hartree

$\begin{array}{lrrr}\mathrm{Ag} & 2.311419 & 1.608187 & -0.543878 \\ \mathrm{Ag} & 4.666103 & 3.030976 & -0.278566 \\ \mathrm{Ag} & 3.804728 & 1.352607 & 1.852666 \\ \mathrm{Ag} & 1.411152 & -0.084601 & 1.559319 \\ \mathrm{Ag} & -1.411150 & -0.084561 & 1.559253 \\ \mathrm{Ag} & 1.445914 & 2.037469 & 3.356321 \\ \mathrm{Ag} & 2.841144 & -0.380725 & 3.903936 \\ \mathrm{Ag} & -1.445899 & 2.037513 & 3.356318 \\ \mathrm{Ag} & 2.370398 & 3.783798 & 1.218640 \\ \mathrm{Ag} & 0.000054 & 4.801499 & -0.210932 \\ \mathrm{Ag} & 2.411635 & 4.127050 & -1.594376 \\ \mathrm{Ag} & -3.804630 & 1.352722 & 1.852636 \\ \mathrm{Ag} & 1.439264 & -2.613315 & 2.782753 \\ \mathrm{Ag} & -1.439281 & -2.613309 & 2.782775 \\ \mathrm{Ag} & -2.841035 & -0.380650 & 3.903979 \\ \mathrm{Ag} & 0.000056 & -0.388538 & 3.981517 \\ \mathrm{Ag} & -2.311422 & 1.608152 & -0.543884 \\ \mathrm{Ag} & 0.000025 & -4.751716 & 1.543498 \\ \mathrm{Ag} & 0.000046 & 2.346330 & 0.965459\end{array}$




$\begin{array}{lrrr}\mathrm{Ag} & -3.805173 & -1.550665 & 1.493752 \\ \mathrm{Ag} & -2.411577 & 4.126935 & -1.594462 \\ \mathrm{Ag} & -4.666104 & 3.031095 & -0.278624 \\ \mathrm{Ag} & -2.370350 & 3.783892 & 1.218608 \\ \mathrm{Ag} & -0.000050 & -1.940691 & -2.596625 \\ \mathrm{Ag} & -0.000066 & -4.439581 & -1.295745 \\ \mathrm{Ag} & -0.000008 & -2.259972 & 0.364120 \\ \mathrm{Ag} & 0.000025 & 2.718242 & -2.045350 \\ \mathrm{Ag} & -2.408997 & -3.409204 & -2.512491 \\ \mathrm{Ag} & 2.408884 & -3.409152 & -2.512679 \\ \mathrm{Ag} & 3.805163 & -1.550787 & 1.493677 \\ \mathrm{Ag} & 2.367096 & -3.778340 & 0.302724 \\ \mathrm{Ag} & 2.316447 & -1.211426 & -0.893300 \\ \mathrm{Ag} & -0.000007 & 0.226277 & -0.865809 \\ \mathrm{Ag} & 4.666557 & -2.662735 & -0.977797 \\ \mathrm{Ag} & 1.504037 & 0.488164 & -3.155377 \\ \mathrm{Ag} & 4.759171 & 0.182150 & -0.604205 \\ \mathrm{Ag} & 3.920427 & -0.955304 & -3.090029 \\ \mathrm{Ag} & 3.915091 & 1.879799 & -2.740130 \\ \mathrm{Ag} & -3.920439 & -0.955364 & -3.089946 \\ \mathrm{Ag} & -2.316508 & -1.211396 & -0.893248 \\ \mathrm{Ag} & -1.504059 & 0.488221 & -3.155399 \\ \mathrm{Ag} & -3.915173 & 1.879779 & -2.740134 \\ \mathrm{Ag} & -4.759174 & 0.182208 & -0.604098 \\ \mathrm{Ag} & -4.666662 & -2.662719 & -0.977668 \\ \mathrm{Ag} & -2.367074 & -3.778311 & 0.302803\end{array}$

Ag46

$E=-6707.37951514$ Hartree

$\begin{array}{llll}\text { Ag } & 1.412788 & -0.000020 & 1.510967\end{array}$

$\begin{array}{llll}\mathrm{Ag} & 3.806105 & 1.461869 & 1.625486\end{array}$

$\begin{array}{llll}\mathrm{Ag} & 4.667429 & -2.868568 & -0.692562\end{array}$

$\begin{array}{llll}\mathrm{Ag} & 2.367491 & -3.818318 & 0.716642\end{array}$

$\begin{array}{llll}\mathrm{Ag} & 3.806134 & -1.461886 & 1.625550\end{array}$

$\begin{array}{llll}\mathrm{Ag} & 2.318062 & -1.419342 & -0.785852\end{array}$

$\begin{array}{llll}\mathrm{Ag} & 4.763162 & -0.000040 & -0.669502\end{array}$

$\begin{array}{lllll}\mathrm{Ag} & 2.409740 & -3.797814 & -2.123721\end{array}$

$\begin{array}{llll}\mathrm{Ag} & 3.919304 & -1.428830 & -2.997369\end{array}$

$\begin{array}{llll}\mathrm{Ag} & 4.667419 & 2.868426 & -0.692601\end{array}$

$\begin{array}{llll}\mathrm{Ag} & 2.367566 & 3.818330 & 0.716490\end{array}$

$\begin{array}{llll}\mathrm{Ag} & 2.318063 & 1.419302 & -0.785825\end{array}$

$\begin{array}{llll}\mathrm{Ag} & 3.919437 & 1.428718 & -2.997409\end{array}$

$\begin{array}{llll}\mathrm{Ag} & 0.000049 & 4.668927 & -0.790588\end{array}$

$\begin{array}{llll}\mathrm{Ag} & -0.000018 & 2.353437 & -2.396372\end{array}$

Ag $\quad-1.505531 \quad-0.000020 \quad-3.242809$

Ag $\quad-2.409754 \quad-3.797647 \quad-2.123901$ 


$\begin{array}{lrrr}\mathrm{Ag} & 0.000017 & -0.000001 & -0.946454 \\ \mathrm{Ag} & 0.000047 & -2.353441 & -2.396268 \\ \mathrm{Ag} & 1.505564 & -0.000006 & -3.242778 \\ \mathrm{Ag} & -0.000041 & -4.668872 & -0.790537 \\ \mathrm{Ag} & 2.409746 & 3.797626 & -2.123847 \\ \mathrm{Ag} & -0.000095 & -4.631650 & 2.066851 \\ \mathrm{Ag} & -2.318076 & -1.419289 & -0.785836 \\ \mathrm{Ag} & 0.000006 & -2.304375 & 0.591030 \\ \mathrm{Ag} & -2.367619 & -3.818355 & 0.716561 \\ \mathrm{Ag} & -3.919386 & -1.428687 & -2.997456 \\ \mathrm{Ag} & -3.806132 & -1.461911 & 1.625527 \\ \mathrm{Ag} & -4.667495 & -2.868464 & -0.692679 \\ \mathrm{Ag} & -3.919286 & 1.428805 & -2.997371 \\ \mathrm{Ag} & -0.000056 & -0.000012 & 3.949580 \\ \mathrm{Ag} & -2.367495 & 3.818365 & 0.716614 \\ \mathrm{Ag} & -1.439224 & 2.357184 & 3.035560 \\ \mathrm{Ag} & 2.838765 & 0.000028 & 3.872709 \\ \mathrm{Ag} & 1.439256 & 2.357269 & 3.035583 \\ \mathrm{Ag} & -2.838742 & 0.000057 & 3.872648 \\ \mathrm{Ag} & 1.439267 & -2.357185 & 3.035564 \\ \mathrm{Ag} & -1.412738 & -0.000022 & 1.510988 \\ \mathrm{Ag} & -2.409708 & 3.797772 & -2.123786 \\ \mathrm{Ag} & -2.318075 & 1.419355 & -0.785809 \\ \mathrm{Ag} & -1.439333 & -2.357232 & 3.035605 \\ \mathrm{Ag} & -4.667442 & 2.868473 & -0.692567 \\ \mathrm{Ag} & -4.763167 & 0.000018 & -0.669506 \\ \mathrm{Ag} & -3.806087 & 1.461879 & 1.625579 \\ \mathrm{Ag} & 0.000054 & 4.631679 & 2.066859 \\ \mathrm{Ag} & 0.000031 & 2.304466 & 0.591011\end{array}$

$\mathrm{Ag} 47$

$E=-6853.21501502$ Hartree

$\begin{array}{lrrr}\mathrm{Ag} & 2.415176 & -3.579934 & -2.569031 \\ \mathrm{Ag} & 1.502779 & 0.304010 & -3.159542 \\ \mathrm{Ag} & -0.000294 & -2.129159 & -2.635794 \\ \mathrm{Ag} & -0.000277 & -2.484297 & 0.323926 \\ \mathrm{Ag} & -2.323793 & -1.406962 & -0.917127 \\ \mathrm{Ag} & -1.502717 & 0.304382 & -3.159537 \\ \mathrm{Ag} & -3.929660 & -1.111147 & -3.110576 \\ \mathrm{Ag} & -0.000626 & -4.638385 & -1.368687 \\ \mathrm{Ag} & -2.416207 & -3.579388 & -2.568863 \\ \mathrm{Ag} & -4.755986 & -0.001636 & -0.614372 \\ \mathrm{Ag} & -4.637255 & 2.849276 & -0.254453 \\ \mathrm{Ag} & -3.890971 & 1.725831 & -2.725793 \\ \mathrm{Ag} & -3.812805 & -1.781677 & 1.461480 \\ \mathrm{Ag} & -2.371283 & -3.992167 & 0.237430\end{array}$




$\begin{array}{lrrr}\mathrm{Ag} & -2.844977 & -0.655982 & 3.888915 \\ \mathrm{Ag} & -4.674378 & -2.849641 & -1.024977 \\ \mathrm{Ag} & -2.306591 & 1.416488 & -0.530216 \\ \mathrm{Ag} & 0.000002 & 0.002800 & -0.872621 \\ \mathrm{Ag} & 0.000309 & 2.512940 & -1.988650 \\ \mathrm{Ag} & -2.364859 & 3.567765 & 1.309048 \\ \mathrm{Ag} & 0.000683 & 5.149978 & -2.668369 \\ \mathrm{Ag} & -2.347221 & 3.994825 & -1.543818 \\ \mathrm{Ag} & -3.803785 & 1.114918 & 1.859642 \\ \mathrm{Ag} & 2.306889 & 1.415932 & -0.530111 \\ \mathrm{Ag} & 0.000258 & 2.081033 & 0.979171 \\ \mathrm{Ag} & 2.365764 & 3.567157 & 1.309211 \\ \mathrm{Ag} & 2.348260 & 3.994334 & -1.543769 \\ \mathrm{Ag} & 0.000537 & 4.161763 & 2.804046 \\ \mathrm{Ag} & -1.444112 & 1.786161 & 3.402574 \\ \mathrm{Ag} & 0.000558 & 4.651791 & 0.003520 \\ \mathrm{Ag} & -1.415552 & -0.325797 & 1.552896 \\ \mathrm{Ag} & 1.415457 & -0.326182 & 1.552916 \\ \mathrm{Ag} & -0.000114 & -0.671465 & 3.966503 \\ \mathrm{Ag} & 3.891392 & 1.725040 & -2.725756 \\ \mathrm{Ag} & 2.323444 & -1.407429 & -0.917171 \\ \mathrm{Ag} & 1.441823 & -2.872561 & 2.734627 \\ \mathrm{Ag} & 3.804062 & 1.113925 & 1.859590 \\ \mathrm{Ag} & 2.844784 & -0.656736 & 3.888905 \\ \mathrm{Ag} & 4.638062 & 2.848134 & -0.254341 \\ \mathrm{Ag} & 3.929375 & -1.112002 & -3.110657 \\ \mathrm{Ag} & 1.444568 & 1.785821 & 3.402551 \\ \mathrm{Ag} & 3.812328 & -1.782704 & 1.461469 \\ \mathrm{Ag} & 4.756005 & -0.002791 & -0.614435 \\ \mathrm{Ag} & 2.370327 & -3.992742 & 0.237402 \\ \mathrm{Ag} & 4.673669 & -2.850749 & -1.025056 \\ \mathrm{Ag} & -1.442443 & -2.872154 & 2.734688 \\ \mathrm{Ag} & -0.000604 & -4.990617 & 1.463211\end{array}$

Ag48

$\mathrm{E}=-6999.01412903$ Hartree

$\begin{array}{lrrr}\mathrm{Ag} & -0.000195 & 1.578195 & 1.545526 \\ \mathrm{Ag} & -2.359741 & 2.993124 & 2.093535 \\ \mathrm{Ag} & -2.369410 & 3.977436 & -0.623362 \\ \mathrm{Ag} & -3.807173 & 0.488463 & 2.179699 \\ \mathrm{Ag} & -1.478305 & 3.420516 & -3.257991 \\ \mathrm{Ag} & -0.000089 & 2.519843 & -1.142320 \\ \mathrm{Ag} & -0.000205 & 5.226008 & -1.668519 \\ \mathrm{Ag} & -0.000248 & 4.333014 & 1.026548 \\ \mathrm{Ag} & -0.000271 & 3.309866 & 3.685906 \\ \mathrm{Ag} & 2.311230 & 1.215319 & -0.108507\end{array}$




$\begin{array}{lrrr}\mathrm{Ag} & 2.369138 & 3.977691 & -0.623204 \\ \mathrm{Ag} & 2.359217 & 2.993351 & 2.093760 \\ \mathrm{Ag} & 4.637561 & 2.579638 & 0.410810 \\ \mathrm{Ag} & 3.812420 & 2.013293 & -2.258456 \\ \mathrm{Ag} & 1.478208 & 3.420717 & -3.257791 \\ \mathrm{Ag} & 1.452955 & 0.858654 & 3.846771 \\ \mathrm{Ag} & -0.000087 & -1.656223 & 3.936439 \\ \mathrm{Ag} & -1.453279 & 0.858555 & 3.846707 \\ \mathrm{Ag} & 2.857237 & -1.624211 & 3.859945 \\ \mathrm{Ag} & 3.806905 & 0.488714 & 2.179940 \\ \mathrm{Ag} & -2.311391 & 1.215105 & -0.108747 \\ \mathrm{Ag} & -4.637868 & 2.579224 & 0.410446 \\ \mathrm{Ag} & -4.745145 & -0.134235 & -0.483034 \\ \mathrm{Ag} & -1.456376 & 0.630426 & -2.900421 \\ \mathrm{Ag} & -3.822880 & -2.277362 & 1.254699 \\ \mathrm{Ag} & -3.892330 & -0.759013 & -3.114242 \\ \mathrm{Ag} & -2.316394 & -1.494303 & -1.004569 \\ \mathrm{Ag} & -2.389950 & -4.210539 & -0.353010 \\ \mathrm{Ag} & -4.677675 & -2.858883 & -1.396106 \\ \mathrm{Ag} & -3.812507 & 2.012826 & -2.258744 \\ \mathrm{Ag} & -2.418042 & -3.317573 & -3.036142 \\ \mathrm{Ag} & 0.000160 & -1.882134 & -2.827792 \\ \mathrm{Ag} & 0.000170 & -2.837949 & 0.049397 \\ \mathrm{Ag} & -2.857317 & -1.624413 & 3.859778 \\ \mathrm{Ag} & 0.000001 & -0.156622 & -0.671330 \\ \mathrm{Ag} & 1.456394 & 0.630594 & -2.900281 \\ \mathrm{Ag} & 2.418762 & -3.317108 & -3.036241 \\ \mathrm{Ag} & 2.316525 & -1.493983 & -1.004516 \\ \mathrm{Ag} & 0.000574 & -4.540766 & -2.071807 \\ \mathrm{Ag} & 4.678103 & -2.858388 & -1.395914 \\ \mathrm{Ag} & 3.892640 & -0.758480 & -3.113997 \\ \mathrm{Ag} & -1.458478 & -3.567595 & 2.329365 \\ \mathrm{Ag} & 1.419576 & -0.881005 & 1.629718 \\ \mathrm{Ag} & 2.390479 & -4.210269 & -0.353066 \\ \mathrm{Ag} & 1.458569 & -3.567488 & 2.329401 \\ \mathrm{Ag} & 3.822940 & -2.277046 & 1.254810 \\ \mathrm{Ag} & 4.745197 & -0.133832 & -0.482766 \\ \mathrm{Ag} & -1.419604 & -0.881153 & 1.629676 \\ & & & \end{array}$

Ag49

$\mathrm{E}=-7144.84903560$ Hartree

$\begin{array}{llll}\mathrm{Ag} & -3.189687 & 2.145151 & 2.185008\end{array}$

$\begin{array}{llll}\mathrm{Ag} & -0.262827 & -3.834968 & 2.184934\end{array}$

$\begin{array}{llll}\mathrm{Ag} & -1.093364 & -1.222950 & 1.588490\end{array}$

$\begin{array}{llll}\mathrm{Ag} & -2.196256 & -2.456601 & 3.787209\end{array}$

$\begin{array}{llll}\mathrm{Ag} & -1.378257 & 4.191061 & 1.164780\end{array}$ 


$\begin{array}{lrrr}\mathrm{Ag} & -1.786375 & -1.998136 & -1.066318 \\ \mathrm{Ag} & -0.974056 & -4.646525 & -0.497113 \\ \mathrm{Ag} & -3.597838 & -4.024479 & -1.500367 \\ \mathrm{Ag} & -3.781636 & -0.689174 & 2.185021 \\ \mathrm{Ag} & -2.940478 & -3.289159 & 1.164760 \\ \mathrm{Ag} & -4.508842 & -1.486459 & -0.497129 \\ \mathrm{Ag} & -2.614479 & 0.546036 & -0.077084 \\ \mathrm{Ag} & -0.837273 & 2.546096 & -1.066245 \\ \mathrm{Ag} & -5.243437 & 1.095087 & 0.488250 \\ \mathrm{Ag} & -4.309353 & 0.899976 & -2.199702 \\ \mathrm{Ag} & -1.325934 & -3.703111 & -3.149513 \\ \mathrm{Ag} & -3.532109 & -1.730835 & -3.149514 \\ \mathrm{Ag} & -2.544074 & 2.999862 & -3.149506 \\ \mathrm{Ag} & -1.686326 & 5.128038 & -1.500376 \\ \mathrm{Ag} & -3.536985 & 3.166923 & -0.497058 \\ \mathrm{Ag} & 0.267117 & 3.924349 & -3.149494 \\ \mathrm{Ag} & -1.647701 & 0.344077 & -2.894098 \\ \mathrm{Ag} & -0.000038 & 0.000028 & -0.694522 \\ \mathrm{Ag} & 0.525821 & -1.598894 & -2.894148 \\ \mathrm{Ag} & -0.512481 & 1.558333 & 1.588564 \\ \mathrm{Ag} & -1.642590 & 0.343029 & 3.863592 \\ \mathrm{Ag} & -1.029406 & 3.130189 & 3.787298 \\ \mathrm{Ag} & 0.967087 & 4.648010 & -0.497046 \\ \mathrm{Ag} & 3.869869 & 0.703377 & -3.149551 \\ \mathrm{Ag} & 2.934115 & 3.281954 & -2.199680 \\ \mathrm{Ag} & 1.780122 & 1.991189 & -0.077060 \\ \mathrm{Ag} & 3.452609 & 1.689769 & 2.185010 \\ \mathrm{Ag} & 4.511117 & 1.479740 & -0.497085 \\ \mathrm{Ag} & 1.118386 & 1.250918 & 3.863622 \\ \mathrm{Ag} & 1.293979 & 3.619537 & 2.185029 \\ \mathrm{Ag} & 4.318755 & -0.901978 & 1.164697 \\ \mathrm{Ag} & 3.570119 & 3.993407 & 0.488228 \\ \mathrm{Ag} & 1.605855 & -0.335375 & 1.588488 \\ \mathrm{Ag} & 3.264974 & -2.193460 & -3.149561 \\ \mathrm{Ag} & 2.623627 & -0.547966 & -1.066267 \\ \mathrm{Ag} & 3.225629 & -0.673697 & 3.787235 \\ \mathrm{Ag} & 3.541831 & -3.161548 & -0.497124 \\ \mathrm{Ag} & 5.284167 & -1.103566 & -1.500347 \\ \mathrm{Ag} & 0.834370 & -2.537230 & -0.077123 \\ \mathrm{Ag} & 2.487660 & -2.930424 & 2.184941 \\ \mathrm{Ag} & 1.375244 & -4.181911 & -2.199765 \\ \mathrm{Ag} & 1.673349 & -5.088494 & 0.488229 \\ \mathrm{Ag} & 1.121806 & 1.254950 & -2.894133 \\ \mathrm{Ag} & 0.524197 & -1.594145 & 3.863544\end{array}$

Ag50 


\begin{tabular}{|c|c|c|c|}
\hline \multicolumn{4}{|c|}{$=-7290.65914361$ Hartree } \\
\hline $\mathrm{Ag}$ & & & \\
\hline $\lg$ & & & \\
\hline$g$ & & & \\
\hline & & & \\
\hline & & & \\
\hline & & & \\
\hline & 37 & $2.3^{\prime}$ & \\
\hline & & & \\
\hline & -1.27 & 2.32 & \\
\hline & & & \\
\hline & -0.26 & & \\
\hline & & 4.6 & \\
\hline & -0.00 & 4.7 & \\
\hline & & 3.80 & \\
\hline & & & \\
\hline & -2.3 & & \\
\hline & & & \\
\hline & -2.0 & & \\
\hline & & & \\
\hline & & -1.4 & \\
\hline & & 1.44 & \\
\hline & & -1.4 & \\
\hline & -3.8 & & \\
\hline & & & \\
\hline & -2.6 & 0.0 & \\
\hline & & -2.31 & \\
\hline & & -0.0 & \\
\hline & & -3.8 & \\
\hline & & & \\
\hline & & -2.3 & \\
\hline & & -2.4 & \\
\hline & & 1.5 & \\
\hline & & -14 & \\
\hline & & -0.000 & \\
\hline & 1400 & 0.00 & \\
\hline & & 1.49 & \\
\hline & -2.5 & -4.67 & \\
\hline & -2.37 & -3.8 & \\
\hline & & -4.752 & \\
\hline & -4.187103 & 0.000 & \\
\hline & & -2.3729 & \\
\hline & & -1.546946 & \\
\hline & & -0.000489 & \\
\hline $\mathrm{Ag}$ & 5.477299 & -0.000940 & -0.824977 \\
\hline & -1.037908 & -1.419084 & 1.579218 \\
\hline
\end{tabular}




$\begin{array}{lrrr}\mathrm{Ag} & -2.098862 & -2.848688 & 3.681464 \\ \mathrm{Ag} & -1.272203 & -2.327141 & -1.110041 \\ \mathrm{Ag} & 0.232309 & -3.805771 & 2.325225 \\ \mathrm{Ag} & 2.552595 & -4.643659 & 0.878187 \\ \mathrm{Ag} & 2.726863 & -2.362318 & 2.594373\end{array}$

\begin{tabular}{lrrc}
$\mathrm{Ag} 51$ & \multicolumn{3}{c}{} \\
$\mathrm{E}=-7436.47128630$ & Hartree & \\
$\mathrm{Ag}$ & 1.264207 & 5.201655 & 0.030657 \\
$\mathrm{Ag}$ & -1.485508 & 4.398249 & -0.064964 \\
$\mathrm{Ag}$ & -2.101272 & 1.664397 & -0.242698 \\
$\mathrm{Ag}$ & 0.230582 & 4.107109 & -2.392382 \\
$\mathrm{Ag}$ & -2.588422 & 3.194131 & -2.469547 \\
$\mathrm{Ag}$ & -2.863441 & 0.738136 & -3.989869 \\
$\mathrm{Ag}$ & -4.186279 & 3.437018 & -0.118199 \\
$\mathrm{Ag}$ & -0.444988 & 1.409479 & -2.709193 \\
$\mathrm{Ag}$ & -2.259311 & -0.745242 & -1.767807 \\
$\mathrm{Ag}$ & -1.954770 & -2.055398 & -4.150970 \\
$\mathrm{Ag}$ & -4.530006 & -1.394750 & -3.184242 \\
$\mathrm{Ag}$ & -4.444454 & 1.046075 & -1.660914 \\
$\mathrm{Ag}$ & 0.621588 & 2.543997 & -0.168775 \\
$\mathrm{Ag}$ & 0.156666 & 3.851642 & 2.288445 \\
$\mathrm{Ag}$ & -0.484679 & 1.133224 & 2.067797 \\
$\mathrm{Ag}$ & -0.948551 & 2.369927 & 4.475186 \\
$\mathrm{Ag}$ & -2.236295 & -0.887390 & 1.081120 \\
$\mathrm{Ag}$ & -2.606986 & 2.957375 & 2.213613 \\
$\mathrm{Ag}$ & -4.555326 & -1.557261 & -0.346634 \\
$\mathrm{Ag}$ & -2.944871 & -3.565617 & 0.994379 \\
$\mathrm{Ag}$ & -4.452405 & -1.672168 & 2.501027 \\
$\mathrm{Ag}$ & -4.392734 & 0.901196 & 1.223233 \\
$\mathrm{Ag}$ & -2.742986 & 0.355880 & 3.551850 \\
$\mathrm{Ag}$ & -2.984772 & -3.434419 & -1.911910 \\
$\mathrm{Ag}$ & -0.208263 & -3.960955 & -2.890274 \\
$\mathrm{Ag}$ & -0.687747 & -2.789462 & -0.478596 \\
$\mathrm{Ag}$ & -0.027412 & -0.107696 & -0.392433 \\
$\mathrm{Ag}$ & 0.397900 & -1.583506 & 1.918158 \\
$\mathrm{Ag}$ & -1.840670 & -2.422255 & 3.402970 \\
$\mathrm{Ag}$ & -0.235545 & -4.281518 & 1.835947 \\
$\mathrm{Ag}$ & 2.632931 & -0.975677 & 3.517776 \\
$\mathrm{Ag}$ & -0.061599 & -0.360920 & 4.412832 \\
$\mathrm{Ag}$ & 4.424511 & -1.059770 & -1.708352 \\
$\mathrm{Ag}$ & 1.728391 & 1.802192 & 3.671268 \\
$\mathrm{Ag}$ & 0.828234 & -3.080067 & 4.181119 \\
$\mathrm{Ag}$ & 2.522703 & -3.409470 & 1.888402 \\
$\mathrm{Ag}$ & 1.467363 & -4.552962 & -0.563153 \\
$\mathrm{Ag}$ & 2.162961 & 0.537860 & 1.198729
\end{tabular}




$\begin{array}{lrrr}\mathrm{Ag} & 4.320165 & -1.249380 & 1.170317 \\ \mathrm{Ag} & 4.349478 & 1.167618 & 2.731613 \\ \mathrm{Ag} & 2.053486 & -1.846927 & -0.412733 \\ \mathrm{Ag} & 2.586710 & -3.092132 & -2.798147 \\ \mathrm{Ag} & 4.149194 & -3.611849 & -0.458610 \\ \mathrm{Ag} & 2.890174 & -0.480591 & -4.020003 \\ \mathrm{Ag} & 1.968713 & 2.307161 & -3.860543 \\ \mathrm{Ag} & 2.236903 & 0.720882 & -1.646345 \\ \mathrm{Ag} & 2.853842 & 3.246154 & 1.418483 \\ \mathrm{Ag} & 4.501418 & 1.375953 & -0.101797 \\ \mathrm{Ag} & 4.527623 & 1.544928 & -2.934589 \\ \mathrm{Ag} & 0.442896 & -1.281349 & -2.871583 \\ \mathrm{Ag} & 2.950652 & 3.446495 & -1.459661\end{array}$

Ag52

$\mathrm{E}=-7582.30984082$ Hartree

$\begin{array}{lrrr}\mathrm{Ag} & -2.314536 & -0.000031 & 1.154646 \\ \mathrm{Ag} & -4.625414 & -0.000124 & 2.629608 \\ \mathrm{Ag} & 0.000014 & 0.000016 & -0.381672 \\ \mathrm{Ag} & -2.351538 & -1.463300 & 3.552851 \\ \mathrm{Ag} & -2.351597 & 1.463055 & 3.552922 \\ \mathrm{Ag} & -0.000023 & 1.430424 & 2.010136 \\ \mathrm{Ag} & -0.000086 & 2.871302 & 4.345039 \\ \mathrm{Ag} & -0.000048 & -1.430478 & 2.010119 \\ \mathrm{Ag} & 0.000018 & -2.871495 & 4.344959 \\ \mathrm{Ag} & 4.760850 & 0.000040 & -0.213346 \\ \mathrm{Ag} & 2.314549 & 0.000043 & 1.154663 \\ \mathrm{Ag} & -0.000104 & -0.000064 & 4.432631 \\ \mathrm{Ag} & 1.453914 & -3.824702 & 2.067322 \\ \mathrm{Ag} & 2.351447 & -1.463249 & 3.552928 \\ \mathrm{Ag} & 3.892213 & 2.376161 & -1.675378 \\ \mathrm{Ag} & -2.867611 & 4.646757 & -0.276028 \\ \mathrm{Ag} & -1.434502 & 2.315707 & -0.314272 \\ \mathrm{Ag} & -0.000072 & 4.728586 & -0.301107 \\ \mathrm{Ag} & -1.453882 & 3.824654 & 2.067373 \\ \mathrm{Ag} & 1.453877 & 3.824659 & 2.067445 \\ \mathrm{Ag} & 1.481949 & 3.794913 & -2.623390 \\ \mathrm{Ag} & 1.434388 & 2.315779 & -0.314276 \\ \mathrm{Ag} & 2.867517 & 4.646774 & -0.275941 \\ \mathrm{Ag} & 3.811730 & 2.369303 & 1.211317 \\ \mathrm{Ag} & 4.625368 & -0.000016 & 2.629699 \\ \mathrm{Ag} & -3.811834 & 2.369194 & 1.211284 \\ \mathrm{Ag} & 2.351428 & 1.463188 & 3.552956 \\ \mathrm{Ag} & -3.811857 & -2.369321 & 1.211185 \\ \mathrm{Ag} & -2.367578 & 0.000033 & -1.699516 \\ \mathrm{Ag} & -3.892175 & 2.376121 & -1.675458\end{array}$




$\begin{array}{lrrr}\mathrm{Ag} & -4.763109 & 0.000046 & -3.052402 \\ \mathrm{Ag} & -1.481950 & 3.794922 & -2.623360 \\ \mathrm{Ag} & -2.545510 & -1.472290 & -3.998993 \\ \mathrm{Ag} & 0.000067 & 1.423584 & -2.785133 \\ \mathrm{Ag} & -2.545525 & 1.472474 & -3.998933 \\ \mathrm{Ag} & -4.760828 & -0.000047 & -0.213472 \\ \mathrm{Ag} & 4.763225 & 0.000055 & -3.052296 \\ \mathrm{Ag} & 1.434470 & -2.315707 & -0.314312 \\ \mathrm{Ag} & 2.545576 & 1.472450 & -3.998917 \\ \mathrm{Ag} & -1.434380 & -2.315698 & -0.314351 \\ \mathrm{Ag} & -1.481880 & -3.794828 & -2.623445 \\ \mathrm{Ag} & 2.367622 & 0.000023 & -1.699475 \\ \mathrm{Ag} & 3.811789 & -2.369222 & 1.211275 \\ \mathrm{Ag} & -1.453845 & -3.824707 & 2.067249 \\ \mathrm{Ag} & 3.892207 & -2.376085 & -1.675394 \\ \mathrm{Ag} & 1.482020 & -3.794836 & -2.623474 \\ \mathrm{Ag} & 2.867633 & -4.646710 & -0.276118 \\ \mathrm{Ag} & -2.867535 & -4.646808 & -0.276163 \\ \mathrm{Ag} & -0.000011 & -4.728591 & -0.301314 \\ \mathrm{Ag} & 0.000023 & -1.423449 & -2.785204 \\ \mathrm{Ag} & -3.892139 & -2.376181 & -1.675544 \\ \mathrm{Ag} & 2.545673 & -1.472324 & -3.998920\end{array}$

Ag53

$\mathrm{E}=-7728.12158444$ Hartree

$\begin{array}{lrrr}\mathrm{Ag} & 2.395356 & 1.443200 & -3.985752 \\ \mathrm{Ag} & 0.000040 & -0.000057 & -0.222308 \\ \mathrm{Ag} & 2.876582 & 4.647433 & -0.165275 \\ \mathrm{Ag} & 1.437041 & 2.325828 & -0.183198 \\ \mathrm{Ag} & 1.479379 & 3.833546 & -2.505208 \\ \mathrm{Ag} & -2.325608 & 0.000088 & -1.623155 \\ \mathrm{Ag} & -2.395080 & -1.443606 & -3.985719 \\ \mathrm{Ag} & -3.834625 & -2.367792 & -1.635712 \\ \mathrm{Ag} & -3.828081 & 2.365745 & 1.286558 \\ \mathrm{Ag} & -1.436735 & 2.326046 & -0.183233 \\ \mathrm{Ag} & -1.461544 & 3.826323 & 2.188817 \\ \mathrm{Ag} & -2.875868 & 4.647857 & -0.165475 \\ \mathrm{Ag} & 1.461906 & 3.826107 & 2.188857 \\ \mathrm{Ag} & 0.000246 & 1.476302 & -2.564633 \\ \mathrm{Ag} & -1.478638 & 3.833740 & -2.505350 \\ \mathrm{Ag} & 0.000365 & 4.736085 & -0.172697 \\ \mathrm{Ag} & -4.664304 & 0.000180 & -3.038183 \\ \mathrm{Ag} & -2.394922 & 1.443563 & -3.985808 \\ \mathrm{Ag} & -4.732615 & 0.000361 & -0.174818 \\ \mathrm{Ag} & -3.834249 & 2.368157 & -1.635941 \\ \mathrm{Ag} & 0.000010 & 1.433511 & 2.137243\end{array}$




$\begin{array}{lrrr}\mathrm{Ag} & -2.364669 & 1.461340 & 3.648540 \\ \mathrm{Ag} & -4.642697 & 0.000408 & 2.693713 \\ \mathrm{Ag} & 0.000057 & 2.866980 & 4.462920 \\ \mathrm{Ag} & -2.323289 & 0.000223 & 1.253887 \\ \mathrm{Ag} & -3.828360 & -2.365028 & 1.286783 \\ \mathrm{Ag} & -2.364799 & -1.460628 & 3.648632 \\ \mathrm{Ag} & 1.478729 & -3.834022 & -2.504920 \\ \mathrm{Ag} & -1.437034 & -2.325830 & -0.183051 \\ \mathrm{Ag} & -0.000025 & -1.476479 & -2.564583 \\ \mathrm{Ag} & -1.479201 & -3.833758 & -2.504976 \\ \mathrm{Ag} & -1.462129 & -3.825908 & 2.189130 \\ \mathrm{Ag} & -0.000388 & -4.736031 & -0.172295 \\ \mathrm{Ag} & -2.876589 & -4.647437 & -0.165119 \\ \mathrm{Ag} & -0.000191 & -1.433275 & 2.137317 \\ \mathrm{Ag} & 1.461399 & -3.826195 & 2.189265 \\ \mathrm{Ag} & 3.828330 & 2.365154 & 1.286861 \\ \mathrm{Ag} & -0.000148 & 0.000158 & 4.549736 \\ \mathrm{Ag} & -0.000375 & -2.866581 & 4.463207 \\ \mathrm{Ag} & 2.364632 & 1.461004 & 3.648650 \\ \mathrm{Ag} & 4.642552 & -0.000233 & 2.693950 \\ \mathrm{Ag} & 2.323186 & -0.000161 & 1.253984 \\ \mathrm{Ag} & 2.875861 & -4.647860 & -0.164920 \\ \mathrm{Ag} & 3.827973 & -2.365548 & 1.286991 \\ \mathrm{Ag} & 2.364384 & -1.461023 & 3.648794 \\ \mathrm{Ag} & 3.834790 & 2.367659 & -1.635634 \\ \mathrm{Ag} & 1.436742 & -2.326048 & -0.183016 \\ \mathrm{Ag} & 2.395187 & -1.443894 & -3.985599 \\ \mathrm{Ag} & 0.000095 & -0.000285 & -4.876284 \\ \mathrm{Ag} & 3.834424 & -2.368366 & -1.635470 \\ \mathrm{Ag} & 2.325687 & -0.000213 & -1.623024 \\ \mathrm{Ag} & 4.664569 & -0.000454 & -3.037961 \\ \mathrm{Ag} & 4.732642 & -0.000284 & -0.174520\end{array}$

Ag54

$E=-7873.96070006$ Hartree

$\begin{array}{lrrr}\mathrm{Ag} & 1.433245 & 0.886568 & -2.095633 \\ \mathrm{Ag} & -1.328722 & -0.853317 & -4.463694 \\ \mathrm{Ag} & 3.869333 & -0.432262 & -2.531501 \\ \mathrm{Ag} & 1.591788 & -0.805940 & -4.326435 \\ \mathrm{Ag} & 2.956084 & 1.718085 & -4.200017 \\ \mathrm{Ag} & -3.778609 & -0.558861 & -2.894517 \\ \mathrm{Ag} & 0.092825 & 1.703180 & -4.416954 \\ \mathrm{Ag} & -2.773837 & 1.624471 & -4.480279 \\ \mathrm{Ag} & -1.441402 & 0.842385 & -2.230367 \\ \mathrm{Ag} & -3.845637 & 2.173276 & -1.902641 \\ \mathrm{Ag} & 2.102257 & 3.161696 & 2.828903\end{array}$




\begin{tabular}{|c|c|c|c|}
\hline g & .187629 & 434020 & \\
\hline & & & \\
\hline & -2.589010 & & \\
\hline & 2.213371 & & \\
\hline & -0.230654 & & 666766 \\
\hline & -0.220037 & & \\
\hline & -0.111896 & & \\
\hline & -2.4 & & \\
\hline & -2.5 & & \\
\hline & -4.8 & $-0 .($ & -0.2 \\
\hline $\mathrm{g}$ & -0.1 & & \\
\hline & -1.4 & 3.5 & -2.8 \\
\hline & & & \\
\hline & 4.4 & 2.8 & \\
\hline g & & & \\
\hline & 2.60 & -1.5 & \\
\hline & & & \\
\hline & 1.2 & -0.7 & \\
\hline & -0.0 & -2. & \\
\hline g & & & \\
\hline & -0.0 & -5 & 1. \\
\hline & -2.3 & -4 & \\
\hline g & -2.2 & -3 . & -2.8 \\
\hline g & -1.6 & -0 . & \\
\hline & -4.6 & -2.7 & -1.2 \\
\hline & & & \\
\hline & -3.9 & -2.2 & \\
\hline & -3.1 & -1. & \\
\hline g & -1.7 & & \\
\hline & -4.0 & & \\
\hline g & -0.2 & -1.6 & 794 \\
\hline & -1.6 & -3.5 & 2.7 \\
\hline g & -0.1 & & 69 \\
\hline & & -4.3 & 242 \\
\hline g & & & \\
\hline & 0.1 & -3.2 & -4.2 \\
\hline ig & & & 0.234598 \\
\hline & 2.337506 & -4.009754 & 0.158967 \\
\hline & & -2.5 & -0.769615 \\
\hline & 3.600622 & 0.652162 & 2.917431 \\
\hline & & -2.0 & \\
\hline $\mathrm{Ag}$ & & -1.626030 & -2.141584 \\
\hline $\mathrm{Ag}$ & 2.448563 & -2.987448 & -2.578254 \\
\hline
\end{tabular}

Ag55

$E=-8019.80281421$ Hartree 


\begin{tabular}{|c|c|c|c|}
\hline$g$ & 1.671476 & -1.412019 & -1.646585 \\
\hline $\mathrm{Ag}$ & 3.334382 & -2.816821 & -3.284811 \\
\hline & 1.305184 & -2.132510 & 1.116953 \\
\hline $\mathrm{Ag}$ & 2.135306 & -1.883571 & 3.782818 \\
\hline & 3.025150 & -3.602203 & -0.538294 \\
\hline $\mathrm{Ag}$ & 2.603723 & -4.254270 & 2.228225 \\
\hline & -0.084693 & -3.635724 & 3.031787 \\
\hline g & -0.602263 & -1.184873 & 4.544276 \\
\hline $\mathrm{Ag}$ & 0.465238 & -4.697960 & 1155 \\
\hline & 0.837446 & -3.965650 & -2.447381 \\
\hline $\mathrm{Ag}$ & -0.795931 & -0.279053 & -2.6 \\
\hline $\mathrm{Ag}$ & 0.889753 & -1.718628 & -4.321067 \\
\hline $\mathrm{Ag}$ & -3.539745 & -0.588053 & -3.088933 \\
\hline $\mathrm{Ag}$ & -1.587756 & -0.556747 & -5.197285 \\
\hline $\mathrm{Ag}$ & -1.670174 & -2.814249 & -3.421655 \\
\hline Ag & -2.135311 & 1.883573 & -3.782819 \\
\hline $\mathrm{Ag}$ & -4.057292 & 1.862886 & $-1.5^{\prime}$ \\
\hline $\mathrm{Ag}$ & -3.109845 & -0.033520 & 3.570081 \\
\hline $\mathrm{Ag}$ & -4.429500 & 1.130576 & 1.232134 \\
\hline $\mathrm{Ag}$ & -2.687067 & -0.299438 & -0.434202 \\
\hline $\mathrm{Ag}$ & -3.592067 & -2.834939 & -1.215284 \\
\hline $\mathrm{Ag}$ & -5.360524 & -0.597416 & -0.866224 \\
\hline Ag & -1.388516 & -1.444908 & 1.866170 \\
\hline $\mathrm{Ag}$ & -2.770030 & -2.882587 & 3.722999 \\
\hline $\mathrm{Ag}$ & -1.690581 & -4.967582 & -1.519334 \\
\hline $\mathrm{Ag}$ & -4.142021 & -1.772791 & \\
\hline $\mathrm{Ag}$ & -2.272435 & -3.999123 & 1.122622 \\
\hline $\mathrm{Ag}$ & -0.847381 & -2.490074 & -0.761567 \\
\hline $\mathrm{Ag}$ & -0.837451 & 3.965652 & 2.447380 \\
\hline $\mathrm{Ag}$ & -2.603728 & 4.254271 & -2.228226 \\
\hline $\mathrm{Ag}$ & -0.465243 & 4.697962 & -0.361156 \\
\hline $\mathrm{Ag}$ & -3.025155 & 3.602205 & 0.538293 \\
\hline $\mathrm{Ag}$ & 5.360520 & 0.597418 & 0.866223 \\
\hline $\mathrm{Ag}$ & 3.592062 & 2.834940 & 1.215283 \\
\hline $\mathrm{Ag}$ & -1.305188 & 2.132512 & -1.116954 \\
\hline $\mathrm{Ag}$ & 0.847465 & 2.490073 & 0.761520 \\
\hline $\mathrm{Ag}$ & 2.272431 & 3.999125 & -1.122623 \\
\hline $\mathrm{Ag}$ & 1.388511 & 1.444910 & -1.866171 \\
\hline $\mathrm{Ag}$ & 0.084688 & 3.635726 & -3.031789 \\
\hline $\mathrm{Ag}$ & 2.770026 & 2.882589 & -3.723000 \\
\hline $\mathrm{Ag}$ & 4.142017 & 1.772793 & -1.455308 \\
\hline $\mathrm{Ag}$ & 4.429495 & -1.130575 & -1.232135 \\
\hline $\mathrm{Ag}$ & 3.109841 & 0.033522 & -3.570082 \\
\hline $\mathrm{Ag}$ & 0.602258 & 1.184875 & -4.544277 \\
\hline $\mathrm{Ag}$ & 1.690576 & 4.967584 & 1.519333 \\
\hline 15 & 1.670170 & 2.814251 & 3.421654 \\
\hline
\end{tabular}




$\begin{array}{lrrr}\mathrm{Ag} & 2.687063 & 0.299440 & 0.434201 \\ \mathrm{Ag} & 4.057287 & -1.862885 & 1.576401 \\ \mathrm{Ag} & 0.795926 & 0.279055 & 2.605281 \\ \mathrm{Ag} & 3.539779 & 0.588007 & 3.089010 \\ \mathrm{Ag} & -0.000002 & 0.000000 & 0.000000 \\ \mathrm{Ag} & -1.671481 & 1.412021 & 1.646583 \\ \mathrm{Ag} & -3.334387 & 2.816823 & 3.284810 \\ \mathrm{Ag} & -0.889757 & 1.718630 & 4.321066 \\ \mathrm{Ag} & 1.587751 & 0.556748 & 5.197284\end{array}$

\begin{tabular}{lrrr}
$\mathrm{Ag} 56$ & \multicolumn{3}{c}{} \\
$\mathrm{E}=-8165.62166579$ & & \\
$\mathrm{Ag}$ & 0.627740 & 2.136642 & 1.634723 \\
$\mathrm{Ag}$ & 2.290649 & -0.214568 & 1.647878 \\
$\mathrm{Ag}$ & 0.120342 & 1.818769 & 4.373097 \\
$\mathrm{Ag}$ & -0.398323 & -0.347541 & 2.668223 \\
$\mathrm{Ag}$ & 2.850157 & 1.953260 & 3.335100 \\
$\mathrm{Ag}$ & 4.457442 & -0.428044 & 3.287185 \\
$\mathrm{Ag}$ & 1.807609 & -0.571164 & 4.385570 \\
$\mathrm{Ag}$ & 2.850037 & -3.864964 & 0.023584 \\
$\mathrm{Ag}$ & 0.120474 & -2.878264 & 3.761378 \\
$\mathrm{Ag}$ & 1.139765 & -4.954921 & 2.060335 \\
$\mathrm{Ag}$ & 1.139594 & 4.261877 & 3.260794 \\
$\mathrm{Ag}$ & 2.850299 & -2.742221 & 2.723559 \\
$\mathrm{Ag}$ & 0.627831 & -2.484066 & 1.032980 \\
$\mathrm{Ag}$ & -2.607783 & -1.923509 & 3.366905 \\
$\mathrm{Ag}$ & -2.607878 & 0.996838 & 3.747254 \\
$\mathrm{Ag}$ & -0.903156 & -0.693405 & 5.323827 \\
$\mathrm{Ag}$ & -1.577229 & 3.513245 & 2.688226 \\
$\mathrm{Ag}$ & 4.456902 & 3.061336 & -1.273306 \\
$\mathrm{Ag}$ & 4.538647 & -1.559294 & 0.648123 \\
$\mathrm{Ag}$ & 2.849597 & 1.911930 & -3.359363 \\
$\mathrm{Ag}$ & 4.538346 & 0.218233 & -1.675124 \\
$\mathrm{Ag}$ & 4.457072 & -2.632903 & -2.014986 \\
$\mathrm{Ag}$ & 1.806956 & 4.083717 & -1.698328 \\
$\mathrm{Ag}$ & 2.290434 & -1.319847 & -1.010082 \\
$\mathrm{Ag}$ & 2.290395 & 1.534618 & -0.638285 \\
$\mathrm{Ag}$ & 2.849921 & 3.730078 & 1.012749 \\
$\mathrm{Ag}$ & 0.120053 & 4.696583 & 0.611912 \\
$\mathrm{Ag}$ & 4.538636 & 1.341451 & 1.025883 \\
$\mathrm{Ag}$ & 0.119685 & -1.818355 & -4.373344 \\
$\mathrm{Ag}$ & 1.138938 & 0.693009 & -5.321372 \\
$\mathrm{Ag}$ & 2.849593 & -0.987571 & -3.737064 \\
$\mathrm{Ag}$ & 1.807017 & -3.512469 & -2.687625 \\
$\mathrm{Ag}$ & 0.627402 & 0.347436 & -2.667867 \\
$\mathrm{Ag}$ & -2.608386 & -1.954042 & -3.348818
\end{tabular}




$\begin{array}{lrrr}\mathrm{Ag} & -0.903769 & -4.263829 & -3.262287 \\ \mathrm{Ag} & -0.398695 & -2.137024 & -1.634985 \\ \mathrm{Ag} & 0.120138 & -4.696629 & -0.611429 \\ \mathrm{Ag} & -4.241485 & -3.050065 & 1.268609 \\ \mathrm{Ag} & -2.061502 & -1.537565 & 0.639486 \\ \mathrm{Ag} & -2.608163 & -3.743630 & -1.009901 \\ \mathrm{Ag} & -1.577027 & -4.084640 & 1.698679 \\ \mathrm{Ag} & -2.061469 & 1.322401 & 1.011972 \\ \mathrm{Ag} & -2.061701 & 0.215021 & -1.651032 \\ \mathrm{Ag} & -1.577726 & 0.571290 & -4.386495 \\ \mathrm{Ag} & -2.608424 & 2.746523 & -2.736776 \\ \mathrm{Ag} & 0.119732 & 2.877824 & -3.761721 \\ \mathrm{Ag} & -4.303526 & -0.223263 & 1.713558 \\ \mathrm{Ag} & -4.303771 & -1.372246 & -1.049514 \\ \mathrm{Ag} & -6.406015 & -0.000010 & 0.000369 \\ \mathrm{Ag} & -4.242073 & 0.426433 & -3.275020 \\ \mathrm{Ag} & -4.303747 & 1.595356 & -0.663061 \\ \mathrm{Ag} & -0.398781 & 2.484459 & -1.033153 \\ \mathrm{Ag} & -2.608255 & 3.877361 & -0.017305 \\ \mathrm{Ag} & 0.112984 & 0.000007 & 0.000036 \\ \mathrm{Ag} & -4.241620 & 2.623188 & 2.007514 \\ \mathrm{Ag} & -0.903885 & 4.957157 & -2.061262\end{array}$

Ag57

$\mathrm{E}=-8311.43885046$ Hartree

$\begin{array}{llll}\text { Ag } & 2.549687 & 2.372278 & 3.378617\end{array}$

$\begin{array}{llll}\mathrm{Ag} & 4.750747 & 2.871313 & -1.082450\end{array}$

$\begin{array}{llll}\mathrm{Ag} & 3.410469 & -1.466499 & -3.189802\end{array}$

$\begin{array}{llll}\mathrm{Ag} & 4.750954 & -2.873126 & -1.076694\end{array}$

$\begin{array}{llll}\mathrm{Ag} & 3.410431 & 1.460388 & -3.192752\end{array}$

$\begin{array}{llll}\mathrm{Ag} & 4.508196 & -1.461409 & 1.411536\end{array}$

$\begin{array}{llll}\mathrm{Ag} & 4.835850 & -0.000880 & -1.099952\end{array}$

$\begin{array}{lllll}\mathrm{Ag} & 2.494782 & -1.439650 & -0.539396\end{array}$

$\begin{array}{llll}\mathrm{Ag} & 4.109021 & 0.003990 & 3.848122\end{array}$

$\begin{array}{llll}\mathrm{Ag} & 2.172078 & 0.001954 & 1.929189\end{array}$

$\begin{array}{llll}\text { Ag } & 0.563171 & 2.331116 & 1.398179\end{array}$

$\begin{array}{llll}\mathrm{Ag} & 2.494711 & 1.438756 & -0.542281\end{array}$

$\begin{array}{llll}\mathrm{Ag} & 0.900186 & 4.652234 & 2.785724\end{array}$

$\begin{array}{llll}\mathrm{Ag} & 4.508048 & 1.464542 & 1.408532\end{array}$

$\begin{array}{llll}\mathrm{Ag} & 2.872651 & 3.831884 & 0.867140\end{array}$

$\begin{array}{llll}\text { Ag } & -1.730997 & 3.837361 & 1.966666\end{array}$

$\begin{array}{lllll}\mathrm{Ag} & -0.434518 & 4.644684 & -2.799381\end{array}$

$\begin{array}{lllll}\mathrm{Ag} & 0.229651 & 4.735107 & -0.006380\end{array}$

$\begin{array}{lllll}\mathrm{Ag} & -0.103950 & 2.328154 & -1.402591\end{array}$

$\begin{array}{lllll}\mathrm{Ag} & 2.194248 & 3.828858 & -1.976802\end{array}$

$\begin{array}{llll}\text { Ag } & 1.093277 & -0.002513 & -2.599236\end{array}$ 


$\begin{array}{lrrr}\mathrm{Ag} & 0.770943 & 2.363852 & -4.067622 \\ \mathrm{Ag} & 1.954176 & -0.005084 & -5.186254 \\ \mathrm{Ag} & 0.771220 & -2.371950 & -4.062941 \\ \mathrm{Ag} & -0.856927 & -0.004623 & -4.612244 \\ \mathrm{Ag} & -2.412610 & 3.829542 & -0.881228 \\ \mathrm{Ag} & -2.072427 & 2.364425 & -3.397518 \\ \mathrm{Ag} & -6.411291 & 1.362838 & -0.019137 \\ \mathrm{Ag} & -4.327817 & 2.880496 & 1.075483 \\ \mathrm{Ag} & -0.286401 & 2.374539 & 4.067696 \\ \mathrm{Ag} & 0.224355 & -0.000059 & 0.001430 \\ \mathrm{Ag} & -2.917959 & 1.490293 & 3.218731 \\ \mathrm{Ag} & -1.463981 & 0.005125 & 5.197377 \\ \mathrm{Ag} & 1.340298 & 0.004638 & 4.603544 \\ \mathrm{Ag} & -2.041886 & 1.447174 & 0.541344 \\ \mathrm{Ag} & -0.434047 & -4.650269 & -2.790087 \\ \mathrm{Ag} & -2.072131 & -2.371312 & -3.392910 \\ \mathrm{Ag} & -3.625646 & -0.004014 & -3.878329 \\ \mathrm{Ag} & -2.412322 & -3.831518 & -0.873635 \\ \mathrm{Ag} & -4.327710 & -2.878542 & 1.081006 \\ \mathrm{Ag} & -4.054019 & -1.456095 & -1.427613 \\ \mathrm{Ag} & -1.705210 & -0.002024 & -1.937967 \\ \mathrm{Ag} & -6.411091 & -1.362768 & -0.016907 \\ \mathrm{Ag} & -4.054337 & 1.452602 & -1.430255 \\ \mathrm{Ag} & 0.229961 & -4.735158 & 0.003037 \\ \mathrm{Ag} & -1.730852 & -3.833596 & 1.974164 \\ \mathrm{Ag} & -0.103808 & -2.331029 & -1.398020 \\ \mathrm{Ag} & -2.041747 & -1.446228 & 0.544229 \\ \mathrm{Ag} & -2.917874 & -1.484091 & 3.221563 \\ \mathrm{Ag} & -4.353668 & 0.001038 & 1.195691 \\ \mathrm{Ag} & -0.628826 & 0.002578 & 2.602205 \\ \mathrm{Ag} & 0.563330 & -2.328348 & 1.402773 \\ \mathrm{Ag} & 2.549887 & -2.365370 & 3.383388 \\ \mathrm{Ag} & 0.900489 & -4.646642 & 2.794984 \\ \mathrm{Ag} & 2.872887 & -3.829887 & 0.874760 \\ \mathrm{Ag} & -0.286309 & -2.366461 & 4.072417 \\ \mathrm{Ag} & 2.194655 & -3.832615 & -1.969141\end{array}$

Ag58

$E=-8457.25974876$ Hartree

$\begin{array}{lrrr}\mathrm{Ag} & 1.836788 & -1.217390 & -1.149217 \\ \mathrm{Ag} & -0.349032 & 1.357066 & 4.538248 \\ \mathrm{Ag} & 0.173906 & 1.957062 & 1.847282 \\ \mathrm{Ag} & 4.037496 & -2.421213 & -2.285448 \\ \mathrm{Ag} & 1.346444 & -1.022715 & 4.309922 \\ \mathrm{Ag} & 4.070700 & -1.658999 & 0.496161 \\ \mathrm{Ag} & 4.037540 & -0.768760 & 3.239487\end{array}$




\begin{tabular}{|c|c|c|c|}
\hline $\mathrm{Ag}$ & 2.377126 & 1.598908 & 3.540209 \\
\hline $\mathrm{Ag}$ & 4.070700 & 1.259119 & 1.188577 \\
\hline & 6.289851 & -1.165320 & -1.099924 \\
\hline g & 6.289867 & -0.369982 & 1.559148 \\
\hline $\mathrm{Ag}$ & 2.377040 & 3.626508 & 1.392235 \\
\hline & 6.289836 & 1.535188 & -0.459126 \\
\hline $\mathrm{Ag}$ & 1.836813 & 1.603838 & -0.479743 \\
\hline & 2.376981 & 2.266393 & -3.154931 \\
\hline $\mathrm{Ag}$ & 4.037628 & 3.189821 & -0.954172 \\
\hline g & 4.070711 & 0.399689 & -1.684864 \\
\hline Ag & 1.346480 & 4.243846 & -1.269379 \\
\hline $\mathrm{Ag}$ & 0.674439 & 3.905432 & 3.686451 \\
\hline $\mathrm{Ag}$ & 2.376981 & -0.607527 & -3.836792 \\
\hline Ag & 1.346307 & -3.221173 & -3.040577 \\
\hline $\mathrm{Ag}$ & -0.349152 & -3.251274 & 3.444809 \\
\hline $\mathrm{Ag}$ & 0.173770 & -2.578365 & 0.771203 \\
\hline $\mathrm{Ag}$ & 1.836809 & -0.386540 & 1.628826 \\
\hline $\mathrm{Ag}$ & -1.369330 & -1.239049 & 5.221765 \\
\hline $\mathrm{Ag}$ & 2.376995 & -3.019117 & 2.444401 \\
\hline $\mathrm{Ag}$ & 0.674151 & -5.14 & 38890 \\
\hline $\mathrm{Ag}$ & 2.376942 & -3.865527 & -0.385405 \\
\hline $\mathrm{Ag}$ & -0.853903 & -0.621107 & 2.617757 \\
\hline $\mathrm{Ag}$ & -0.853901 & 2.577669 & 71003 \\
\hline $\mathrm{Ag}$ & -1.369252 & 5.141670 & -1.537837 \\
\hline $\mathrm{Ag}$ & -2.034176 & 5780 & 35494 \\
\hline $\mathrm{Ag}$ & -0.349028 & 4.608962 & 1.093221 \\
\hline $\mathrm{Ag}$ & -3.077259 & 3.846337 & 75429 \\
\hline $\mathrm{Ag}$ & -3.077308 & 3.008540 & -2.425549 \\
\hline $\mathrm{Ag}$ & -0.349194 & -1.357705 & -4.538037 \\
\hline $\mathrm{Ag}$ & -2.034354 & 1.020939 & -4.302682 \\
\hline $\mathrm{Ag}$ & -0.349061 & 3.251695 & -3.444436 \\
\hline $\mathrm{Ag}$ & 0.173743 & 0.621321 & -2.618506 \\
\hline $\mathrm{Ag}$ & 0.674243 & 1.239785 & -5.225419 \\
\hline $\mathrm{Ag}$ & -4.764114 & -1.227598 & -1.158791 \\
\hline $\mathrm{Ag}$ & -4.683284 & 0.765294 & -3.224696 \\
\hline $\mathrm{Ag}$ & -2.514671 & 0.383552 & -1.616211 \\
\hline $\mathrm{Ag}$ & -4.683304 & -3.175250 & 0.949770 \\
\hline $\mathrm{Ag}$ & -2.034333 & -4.236616 & 1.267132 \\
\hline $\mathrm{Ag}$ & -3.077425 & -3.604843 & -1.392611 \\
\hline $\mathrm{Ag}$ & -0.853951 & -1.956513 & -1.846777 \\
\hline $\mathrm{Ag}$ & -3.077497 & -1.598042 & -3.518639 \\
\hline $\mathrm{Ag}$ & -4.683155 & 2.410260 & 2.275152 \\
\hline $\mathrm{Ag}$ & -2.514616 & 1.208035 & 1.140284 \\
\hline $\mathrm{Ag}$ & -2.514647 & -1.591458 & 0.476002 \\
\hline $\mathrm{Ag}$ & -4.764029 & -0.389605 & 1.642753 \\
\hline $\mathrm{Ag}$ & -4.764065 & 1.617517 & -0.483706 \\
\hline
\end{tabular}




$$
\begin{array}{lrrc}
\mathrm{Ag} & -3.077411 & -2.248135 & 3.143331 \\
\mathrm{Ag} & -0.332847 & -0.000033 & -0.000010 \\
\mathrm{Ag} & -1.369489 & -3.902658 & -3.683815 \\
\mathrm{Ag} & -3.077306 & 0.596404 & 3.818295 \\
\mathrm{Ag} & -0.349193 & -4.608781 & -1.093930
\end{array}
$$

Ag59

$\mathrm{E}=-8603.06842509$ Hartree

$\begin{array}{lrrc}\mathrm{Ag} & -2.304385 & 2.281656 & 4.573728 \\ \mathrm{Ag} & -3.753418 & 2.602686 & 2.098126 \\ \mathrm{Ag} & -0.225731 & 2.751464 & 0.203494 \\ \mathrm{Ag} & -2.739000 & 1.429484 & -0.230056 \\ \mathrm{Ag} & 1.596312 & 3.783065 & 2.023142 \\ \mathrm{Ag} & -2.607776 & 4.221578 & -0.043080 \\ \mathrm{Ag} & -1.227449 & 3.948695 & 2.534995 \\ \mathrm{Ag} & -0.077848 & 5.468439 & 0.382038 \\ \mathrm{Ag} & -2.974421 & -3.909055 & 0.890989 \\ \mathrm{Ag} & -3.671754 & 0.030311 & 3.420616 \\ \mathrm{Ag} & -4.920867 & -2.231345 & 2.150186 \\ \mathrm{Ag} & -5.070457 & 0.308918 & 0.858450 \\ \mathrm{Ag} & -4.595853 & -2.107390 & -0.707735 \\ \mathrm{Ag} & -4.663483 & 0.474549 & -2.039527 \\ \mathrm{Ag} & -2.263972 & -0.954510 & -1.765660 \\ \mathrm{Ag} & -3.162011 & 2.884513 & -2.605360 \\ \mathrm{Ag} & -5.069928 & 2.839702 & -0.460155 \\ \mathrm{Ag} & -1.074696 & -0.287416 & 4.693351 \\ \mathrm{Ag} & -2.394205 & -2.587974 & 3.450181 \\ \mathrm{Ag} & -1.340363 & 1.148086 & 2.289380 \\ \mathrm{Ag} & 0.551580 & 2.100077 & 4.064954 \\ \mathrm{Ag} & -2.664078 & -1.119798 & 1.078464 \\ \mathrm{Ag} & -2.553657 & -3.737542 & -2.008043 \\ \mathrm{Ag} & 1.767660 & -0.485217 & 4.166965 \\ \mathrm{Ag} & -0.090094 & -1.428787 & 2.328955 \\ \mathrm{Ag} & 0.198446 & -2.859869 & 4.632174 \\ \mathrm{Ag} & 1.604908 & 0.920518 & 1.685749 \\ \mathrm{Ag} & 2.199279 & -3.063327 & 2.610121 \\ \mathrm{Ag} & -0.380222 & -4.233057 & 2.156292 \\ \mathrm{Ag} & -0.669691 & -2.734913 & -0.190584 \\ \mathrm{Ag} & -1.293570 & -2.118603 & -4.117401 \\ \mathrm{Ag} & 0.306237 & -3.934279 & -2.524440 \\ \mathrm{Ag} & -0.921234 & -5.446852 & -0.397127 \\ \mathrm{Ag} & 1.660195 & -4.317816 & 0.044015 \\ \mathrm{Ag} & 1.853950 & -1.551701 & 0.281308 \\ \mathrm{Ag} & -2.636955 & 0.458344 & -4.167979 \\ \mathrm{Ag} & -4.112693 & -1.906077 & -3.539391 \\ \mathrm{Ag} & 1.957909 & 4.074564 & -1.033015\end{array}$




$\begin{array}{rrrr}\mathrm{Ag} & 3.015190 & -0.109667 & -3.210367 \\ \mathrm{Ag} & 4.078116 & 2.311138 & -2.173756 \\ \mathrm{Ag} & 3.698256 & 2.627567 & 0.610551 \\ \mathrm{Ag} & -1.109007 & 2.820873 & -4.642175 \\ \mathrm{Ag} & -0.632266 & 4.219853 & -2.180877 \\ \mathrm{Ag} & 1.507007 & 2.624320 & -3.513331 \\ \mathrm{Ag} & 1.751911 & 1.275727 & -1.129817 \\ \mathrm{Ag} & -0.784990 & 1.432691 & -2.320622 \\ \mathrm{Ag} & 0.288384 & 0.287530 & -4.634670 \\ \mathrm{Ag} & 5.707007 & 1.219494 & 1.799598 \\ \mathrm{Ag} & 3.880970 & -0.811432 & 2.341167 \\ \mathrm{Ag} & 3.427658 & 1.871300 & 3.412092 \\ \mathrm{Ag} & 1.552697 & -2.263958 & -4.537164 \\ \mathrm{Ag} & 0.577793 & -1.138720 & -2.258788 \\ \mathrm{Ag} & 6.351603 & -1.172384 & 0.616891 \\ \mathrm{Ag} & 5.401738 & -1.430598 & -2.034346 \\ \mathrm{Ag} & 6.325453 & 1.149011 & -0.960091 \\ \mathrm{Ag} & 2.898476 & -2.731462 & -2.075600 \\ \mathrm{Ag} & 4.232287 & -2.909893 & 0.384930 \\ \mathrm{Ag} & 3.985232 & 0.008688 & -0.293145 \\ \mathrm{Ag} & -0.390177 & 0.008799 & 0.011403\end{array}$

Ag60

$E=-8748.88599516$ Hartree

$\begin{array}{lrrr}\mathrm{Ag} & -0.561412 & -0.004566 & 4.738596 \\ \mathrm{Ag} & -0.721782 & -1.432845 & 2.333188 \\ \mathrm{Ag} & 0.642134 & -2.475741 & 0.000068 \\ \mathrm{Ag} & 1.765579 & -4.956009 & 0.000231 \\ \mathrm{Ag} & 0.458965 & -3.971409 & 2.364536 \\ \mathrm{Ag} & -0.904998 & -2.860332 & 4.648612 \\ \mathrm{Ag} & 1.222979 & 2.116249 & -0.000106 \\ \mathrm{Ag} & 1.374680 & 3.623554 & 2.373653 \\ \mathrm{Ag} & 1.956960 & 1.157942 & 3.891937 \\ \mathrm{Ag} & 1.616229 & -1.760857 & 3.864733 \\ \mathrm{Ag} & -0.208630 & 2.856820 & 4.655897 \\ \mathrm{Ag} & -0.364398 & 1.434512 & 2.334271 \\ \mathrm{Ag} & 3.562641 & 1.895298 & -1.569230 \\ \mathrm{Ag} & 1.752261 & -0.290348 & -1.502150 \\ \mathrm{Ag} & 4.101010 & -0.573930 & -2.960407 \\ \mathrm{Ag} & 3.027409 & -2.823239 & -1.472639 \\ \mathrm{Ag} & 5.784119 & -2.143607 & -1.376264 \\ \mathrm{Ag} & 4.101054 & -0.573597 & 2.960390 \\ \mathrm{Ag} & 1.752285 & -0.290251 & 1.502217 \\ \mathrm{Ag} & 3.027420 & -2.823170 & 1.472884 \\ \mathrm{Ag} & 5.784141 & -2.143589 & 1.376407 \\ \mathrm{Ag} & 4.046535 & -0.496826 & 0.000032\end{array}$




$\begin{array}{lrrr}\mathrm{Ag} & 6.141151 & 0.646710 & 1.403442 \\ \mathrm{Ag} & 3.562661 & 1.895388 & 1.569009 \\ \mathrm{Ag} & 6.141122 & 0.646694 & -1.403540 \\ \mathrm{Ag} & 5.550552 & 2.991364 & -0.000136 \\ \mathrm{Ag} & 2.969389 & 4.232189 & -0.000138 \\ \mathrm{Ag} & -1.093632 & -4.688867 & 0.000187 \\ \mathrm{Ag} & -2.454829 & -3.631435 & -2.365033 \\ \mathrm{Ag} & 1.616257 & -1.761250 & -3.864585 \\ \mathrm{Ag} & 0.458913 & -3.971581 & -2.364264 \\ \mathrm{Ag} & -0.520379 & 0.001319 & -0.000010 \\ \mathrm{Ag} & -0.364451 & 1.434268 & -2.334347 \\ \mathrm{Ag} & 1.956947 & 1.157663 & -3.892088 \\ \mathrm{Ag} & -2.235597 & -2.136704 & 0.000114 \\ \mathrm{Ag} & -2.454832 & -3.631302 & 2.365378 \\ \mathrm{Ag} & -0.905052 & -2.860760 & -4.648301 \\ \mathrm{Ag} & -0.721842 & -1.433059 & -2.333096 \\ \mathrm{Ag} & -0.561501 & -0.004960 & -4.738606 \\ \mathrm{Ag} & -3.076908 & -1.166541 & -3.827174 \\ \mathrm{Ag} & -0.208665 & 2.856371 & -4.656086 \\ \mathrm{Ag} & -2.717074 & 1.743082 & -3.828605 \\ \mathrm{Ag} & -4.619234 & -1.882615 & -1.458526 \\ \mathrm{Ag} & -3.942193 & -4.264271 & 0.000219 \\ \mathrm{Ag} & -2.717096 & 1.743506 & 3.828464 \\ \mathrm{Ag} & -5.149204 & 0.570150 & 2.863490 \\ \mathrm{Ag} & -4.036090 & 2.820446 & -1.457202 \\ \mathrm{Ag} & -3.076884 & -1.166209 & 3.827288 \\ \mathrm{Ag} & -4.619188 & -1.882524 & 1.458745 \\ \mathrm{Ag} & -1.658018 & 2.489686 & -0.000086 \\ \mathrm{Ag} & 0.077956 & 4.680296 & -0.000223 \\ \mathrm{Ag} & -4.036100 & 2.820485 & 1.456925 \\ \mathrm{Ag} & -1.518687 & 3.987912 & 2.368013 \\ \mathrm{Ag} & -2.793398 & 4.967241 & -0.000182 \\ \mathrm{Ag} & 1.374706 & 3.623353 & -2.373940 \\ \mathrm{Ag} & -1.518692 & 3.987651 & -2.368372 \\ \mathrm{Ag} & -5.232128 & 0.580413 & -0.000031 \\ \mathrm{Ag} & -2.841995 & 0.285895 & -1.436259 \\ \mathrm{Ag} & -5.149214 & 0.569921 & -2.863508 \\ \mathrm{Ag} & -2.841951 & 0.286015 & 1.436210\end{array}$

Ag61
$\begin{array}{cccc}\mathrm{E}=-8894.69936032 & \text { Hartree } & \\ \mathrm{Ag} & 1.714210 & 1.464580 & -3.893633 \\ \mathrm{Ag} & 2.358592 & 4.619775 & 0.000262 \\ \mathrm{Ag} & 5.080019 & -3.693970 & -0.000234 \\ \mathrm{Ag} & 4.054253 & 0.000202 & -2.972970 \\ \mathrm{Ag} & 3.228359 & 2.364430 & -1.551245\end{array}$




\begin{tabular}{|c|c|c|c|}
\hline & 3.995062 & 0.000005 & 0.000021 \\
\hline g & 3.228413 & -2.364401 & 1.551213 \\
\hline & 5.926913 & -1.420118 & -1.397244 \\
\hline & 3.228340 & 2.364167 & 1.551554 \\
\hline & 5.926873 & -1.420255 & 1.397019 \\
\hline & 5.926875 & 1.420378 & -1.397084 \\
\hline g & 5.926903 & 1.420117 & 1.397188 \\
\hline & 5.079948 & 3.694023 & 0.000185 \\
\hline g & 0.844742 & -3.826083 & -2.371618 \\
\hline & 0.866041 & -2.316407 & -0.000179 \\
\hline$g$ & 1.685759 & 0.000095 & -1.507835 \\
\hline & 1.685763 & -0.000099 & 1.507892 \\
\hline g & 2.358710 & -4.619749 & -0.000432 \\
\hline g & 4.054293 & -0.000247 & 2.972959 \\
\hline $\mathrm{Ag}$ & -2.076565 & -3.84 & -2.365658 \\
\hline g & -0.625229 & -1.445478 & -2.334951 \\
\hline $\mathrm{g}$ & 0.844688 & 3.826472 & -2.371031 \\
\hline$g$ & 3.228349 & -2.36 & -1.551615 \\
\hline$g$ & 0.866013 & 2.316337 & 0.000173 \\
\hline $\mathrm{Ag}$ & -0.625201 & 1.44 & -2.33 \\
\hline$g$ & -0.644673 & -2.881217 & -4.653846 \\
\hline$g$ & -0.644680 & 2.88 & -4.6 \\
\hline $\mathrm{g}$ & -3.000277 & 1.468 & -3.824948 \\
\hline $\lg$ & -2.076564 & 3.84 & -2.3 \\
\hline $\lg$ & -3.000360 & $-1.46^{\prime}$ & -3.825207 \\
\hline & 1.714266 & -1.46 & -3.893780 \\
\hline $\mathrm{Ag}$ & -0.655563 & 0.00 & -4.74 \\
\hline$g$ & -0.599709 & 0.000008 & -0.000031 \\
\hline $\mathrm{Ag}$ & -0.574829 & -4.721273 & -0.000408 \\
\hline g & -2.034225 & -2.333266 & -0.000185 \\
\hline Ag & -4.435039 & -2.370950 & -1.455580 \\
\hline$g$ & -3.462236 & -4.654992 & -0.000391 \\
\hline Ag & -0.625193 & -1.445860 & 2.334720 \\
\hline $\mathrm{lg}$ & 0.844769 & -3.826452 & 2.370981 \\
\hline $\mathrm{Ag}$ & -3.000204 & -1.468432 & 3.824937 \\
\hline $\mathrm{Ag}$ & -2.939740 & -0.000150 & 1.435062 \\
\hline $\mathrm{Ag}$ & -2.076551 & -3.841551 & 2.365077 \\
\hline $\mathrm{Ag}$ & -4.434984 & -2.371242 & 1.455206 \\
\hline $\mathrm{Ag}$ & -0.644656 & -2.882045 & 4.653382 \\
\hline $\mathrm{Ag}$ & 1.714343 & 1.463979 & 3.893773 \\
\hline $\mathrm{Ag}$ & 1.714316 & -1.464626 & 3.893596 \\
\hline $\mathrm{Ag}$ & -0.655476 & -0.000403 & 4.741088 \\
\hline $\mathrm{Ag}$ & -0.644580 & 2.881197 & 4.653844 \\
\hline $\mathrm{Ag}$ & -0.625137 & 1.445498 & 2.335009 \\
\hline $\mathrm{Ag}$ & -5.266611 & -0.000252 & 2.860233 \\
\hline $\mathrm{Ag}$ & -3.000259 & 1.467752 & 3.825229 \\
\hline
\end{tabular}




$\begin{array}{lrrr}\mathrm{Ag} & -2.034136 & 2.333251 & 0.000206 \\ \mathrm{Ag} & -4.435046 & 2.371267 & -1.455234 \\ \mathrm{Ag} & -3.462169 & 4.655053 & 0.000433 \\ \mathrm{Ag} & -5.266675 & 0.000228 & -2.860093 \\ \mathrm{Ag} & -4.434948 & 2.371010 & 1.455716 \\ \mathrm{Ag} & -2.076535 & 3.841105 & 2.365705 \\ \mathrm{Ag} & -5.348918 & 0.000009 & 0.000024 \\ \mathrm{Ag} & 0.844729 & 3.826035 & 2.371640 \\ \mathrm{Ag} & -0.574835 & 4.721365 & 0.000394 \\ \mathrm{Ag} & -2.939737 & 0.000153 & -1.435016\end{array}$

Ag62

$E=-9040.51166256$ Hartree

$\begin{array}{rrrr}\mathrm{Ag} & 2.203413 & 3.839522 & 2.365020 \\ \mathrm{Ag} & 0.747587 & 1.444369 & 2.333376 \\ \mathrm{Ag} & -1.589562 & 1.462951 & 3.886365 \\ \mathrm{Ag} & -2.223827 & 4.628039 & -0.000184 \\ \mathrm{Ag} & 0.707092 & 4.725408 & -0.000163 \\ \mathrm{Ag} & -1.571606 & 0.000074 & 1.492743 \\ \mathrm{Ag} & 0.768618 & 2.880695 & 4.652493 \\ \mathrm{Ag} & -3.096663 & 2.368119 & 1.544741 \\ \mathrm{Ag} & -0.715864 & 3.830302 & 2.371483 \\ \mathrm{Ag} & -5.811491 & 1.456327 & 1.428645 \\ \mathrm{Ag} & -4.943728 & 3.714778 & -0.000165 \\ \mathrm{Ag} & -0.733171 & 2.321975 & -0.000094 \\ \mathrm{Ag} & -3.096615 & -2.368111 & 1.544904 \\ \mathrm{Ag} & -5.811489 & -1.456254 & 1.428812 \\ \mathrm{Ag} & -3.926238 & 0.000039 & 2.972234 \\ \mathrm{Ag} & 3.123996 & 1.467779 & 3.824353 \\ \mathrm{Ag} & 2.162237 & 2.332769 & -0.000062 \\ \mathrm{Ag} & 0.724738 & -0.000026 & -0.000041 \\ \mathrm{Ag} & 0.779601 & 0.000137 & 4.740843 \\ \mathrm{Ag} & -0.715795 & -3.830131 & 2.371734 \\ \mathrm{Ag} & 0.747705 & -1.444129 & 2.333405 \\ \mathrm{Ag} & 0.768711 & -2.880398 & 4.652682 \\ \mathrm{Ag} & -1.589503 & -1.462741 & 3.886458 \\ \mathrm{Ag} & 3.124053 & -1.467474 & 3.824476 \\ \mathrm{Ag} & 4.562588 & -2.370217 & 1.456028 \\ \mathrm{Ag} & 5.391768 & 0.000225 & 2.860980 \\ \mathrm{Ag} & 3.065007 & 0.000076 & 1.435020 \\ \mathrm{Ag} & 5.474512 & 0.000088 & -0.000005 \\ \mathrm{Ag} & 4.562427 & 2.370482 & 1.455850 \\ \mathrm{Ag} & 3.591833 & 4.653905 & -0.000169 \\ \mathrm{Ag} & 2.203508 & -3.839273 & 2.365379 \\ \mathrm{Ag} & -5.811461 & 1.456253 & -1.428776 \\ \mathrm{Ag} & -7.653651 & 0.000070 & -0.000014\end{array}$




$\begin{array}{lrrr}\mathrm{Ag} & -5.811554 & -1.456359 & -1.428709 \\ \mathrm{Ag} & -3.926236 & -0.000160 & -2.972180 \\ \mathrm{Ag} & -4.943805 & -3.714761 & 0.000101 \\ \mathrm{Ag} & -3.096638 & 2.368002 & -1.544910 \\ \mathrm{Ag} & -1.589591 & 1.462575 & -3.886494 \\ \mathrm{Ag} & -1.589536 & -1.463084 & -3.886308 \\ \mathrm{Ag} & -1.571593 & -0.000142 & -1.492768 \\ \mathrm{Ag} & -3.096594 & -2.368195 & -1.544653 \\ \mathrm{Ag} & -3.915153 & 0.000031 & -0.000010 \\ \mathrm{Ag} & -2.223782 & -4.627999 & 0.000149 \\ \mathrm{Ag} & 0.707190 & -4.725461 & 0.000171 \\ \mathrm{Ag} & -0.715784 & -3.830302 & -2.371478 \\ \mathrm{Ag} & -0.733164 & -2.321935 & 0.000090 \\ \mathrm{Ag} & 0.747587 & 1.444097 & -2.333448 \\ \mathrm{Ag} & -0.715855 & 3.830004 & -2.371789 \\ \mathrm{Ag} & 0.768683 & 2.880292 & -4.652706 \\ \mathrm{Ag} & 2.203386 & 3.839331 & -2.365426 \\ \mathrm{Ag} & 4.562496 & 2.370359 & -1.455997 \\ \mathrm{Ag} & 5.391739 & -0.000037 & -2.861057 \\ \mathrm{Ag} & 3.064965 & -0.000035 & -1.435045 \\ \mathrm{Ag} & 3.124014 & 1.467491 & -3.824438 \\ \mathrm{Ag} & 0.779547 & -0.000198 & -4.740802 \\ \mathrm{Ag} & 2.162248 & -2.332639 & 0.000079 \\ \mathrm{Ag} & 3.592015 & -4.653805 & 0.000224 \\ \mathrm{Ag} & 2.203581 & -3.839467 & -2.365061 \\ \mathrm{Ag} & 3.124071 & -1.467762 & -3.824315 \\ \mathrm{Ag} & 4.562557 & -2.370335 & -1.455826 \\ \mathrm{Ag} & 0.768772 & -2.880767 & -4.652422 \\ \mathrm{Ag} & 0.747701 & -1.444367 & -2.333325\end{array}$

Ag63

$E=-9186.31882781$ Hartree

$\begin{array}{lrrr}\mathrm{Ag} & -3.474568 & -1.821314 & -0.192418 \\ \mathrm{Ag} & -2.845459 & -3.662702 & 2.020065 \\ \mathrm{Ag} & -3.177622 & -0.868924 & 2.585788 \\ \mathrm{Ag} & -6.025730 & -0.595655 & -0.433570 \\ \mathrm{Ag} & -5.667232 & 0.417597 & 2.156734 \\ \mathrm{Ag} & -3.424404 & 1.952741 & 3.041072 \\ \mathrm{Ag} & -1.673535 & -0.041351 & -1.274477 \\ \mathrm{Ag} & -3.745474 & 0.973545 & 0.265546 \\ \mathrm{Ag} & -5.628538 & 2.098851 & -1.387736 \\ \mathrm{Ag} & -4.197998 & -0.081587 & -2.499141 \\ \mathrm{Ag} & -5.405304 & -2.382416 & 1.665541 \\ \mathrm{Ag} & -4.470573 & -4.384774 & -0.117049 \\ \mathrm{Ag} & -1.003186 & -1.841505 & 1.020524 \\ \mathrm{Ag} & 0.875003 & -0.475800 & 2.707595\end{array}$




\begin{tabular}{|c|c|c|c|}
\hline $\lg$ & -5.151096 & -2.683811 & -2.174660 \\
\hline$g$ & -0.924618 & -2.349982 & 3.771460 \\
\hline & -1.220426 & 0.510529 & 4.296877 \\
\hline & -5.243629 & 3.089079 & 1.209469 \\
\hline g & 0.988130 & -0.952226 & 5.391474 \\
\hline & 3.846799 & 2.236988 & 2.807379 \\
\hline $\mathrm{Ag}$ & 1.875417 & 4.287954 & 3.204893 \\
\hline & 1.311697 & 2.151318 & 1.607257 \\
\hline $\mathrm{Ag}$ & -1.297943 & 0.974143 & 1.537746 \\
\hline & 1.449497 & 1.698114 & 4.377987 \\
\hline $\mathrm{Ag}$ & -2.408567 & 3.482995 & 0.814048 \\
\hline$g$ & -0.763913 & 3.173179 & 5520 \\
\hline $\mathrm{Ag}$ & -4.230189 & 4.491093 & -0.9 \\
\hline $\mathrm{Ag}$ & -0.320744 & 2.41 & -0.771403 \\
\hline $\lg$ & 0.266369 & 4.63 & 0.846718 \\
\hline $\mathrm{Ag}$ & 3.122053 & 4.05 & \\
\hline $\mathrm{Ag}$ & -1.411246 & 4.81 & -1.534714 \\
\hline g & -2.831238 & 2.41 & -2.10 \\
\hline Ag & 0.694179 & 0.478111 & -2.709400 \\
\hline $\mathrm{Ag}$ & 1.475876 & 4.31 & -1.7 \\
\hline $\mathrm{Ag}$ & -0.367552 & 2.92 & -3.5 \\
\hline $\mathrm{Ag}$ & -0.354871 & -4.3 & 5068 \\
\hline $\mathrm{Ag}$ & 0.623724 & 0.94 & -5.35 \\
\hline $\mathrm{Ag}$ & -2.370836 & -2.293713 & -2.851266 \\
\hline $\mathrm{Ag}$ & 2.792655 & -0.5 & 57333 \\
\hline $\lg$ & 0.115391 & -1.716460 & -4.356766 \\
\hline $\mathrm{Ag}$ & -1.802929 & 0.411093 & -4.022354 \\
\hline $\mathrm{Ag}$ & 2.823363 & -0.990504 & -1.486566 \\
\hline Ag & 2.485708 & 2.359698 & -3.742345 \\
\hline $\mathrm{Ag}$ & 4.661363 & 0.86 & -2.50 \\
\hline $\mathrm{Ag}$ & 4.286526 & 3.672556 & -1.95 \\
\hline $\mathrm{Ag}$ & 4.895959 & -1.971640 & -2.964416 \\
\hline $\mathrm{Ag}$ & 2.517733 & 1.843299 & -0.980520 \\
\hline $\mathrm{Ag}$ & 5.073870 & 1.921511 & 0.178647 \\
\hline $\mathrm{Ag}$ & 0.176234 & -2.165513 & -1.592032 \\
\hline $\mathrm{Ag}$ & -1.671337 & -4.071415 & -0.616141 \\
\hline $\mathrm{Ag}$ & 2.294924 & -3.195154 & -3.126126 \\
\hline $\mathrm{Ag}$ & 2.818033 & -4.814871 & 1.565937 \\
\hline $\mathrm{Ag}$ & -0.002785 & -4.320198 & 1.809463 \\
\hline $\mathrm{Ag}$ & 1.225553 & -4.641003 & -0.816278 \\
\hline $\mathrm{Ag}$ & 0.745294 & 0.001200 & -0.000325 \\
\hline $\mathrm{Ag}$ & 1.791734 & -2.412808 & 0.786507 \\
\hline $\mathrm{Ag}$ & 3.912393 & -3.459116 & -0.714953 \\
\hline $\mathrm{Ag}$ & 5.380795 & -0.950550 & -0.333193 \\
\hline $\mathrm{Ag}$ & 3.397830 & -0.434306 & 3.922266 \\
\hline $\mathrm{Ag}$ & 4.330137 & -2.399680 & 1.975263 \\
\hline
\end{tabular}




$\begin{array}{llll}\mathrm{Ag} & 5.706077 & 0.104031 & 2.311845 \\ \mathrm{Ag} & 1.920678 & -2.938980 & 3.538568 \\ \mathrm{Ag} & 3.232551 & 0.052241 & 1.161386\end{array}$

Ag64

$\mathrm{E}=-9332.13430134$ Hartree

$\begin{array}{llll}\text { Ag } & -1.067084 & -1.435718 & 1.399839\end{array}$

$\begin{array}{llll}\mathrm{Ag} & -0.911647 & -1.457269 & 4.193081\end{array}$

$\begin{array}{llll}\mathrm{Ag} & 1.862059 & -2.365279 & 3.987274\end{array}$

$\begin{array}{llll}\mathrm{Ag} & -2.622069 & 2.361769 & -2.380930\end{array}$

$\begin{array}{llll}\text { Ag } & -5.401399 & 2.460437 & -1.548132\end{array}$

$\begin{array}{llll}\mathrm{Ag} & -3.566238 & 1.435787 & 0.223421\end{array}$

$\begin{array}{llll}\mathrm{Ag} & -0.056769 & 2.344255 & -1.155675\end{array}$

$\begin{array}{lllll}\mathrm{Ag} & 1.865499 & 3.843222 & -2.534074\end{array}$

$\begin{array}{lllll}\mathrm{Ag} & 4.588258 & 2.887877 & -2.672104\end{array}$

$\begin{array}{llll}\mathrm{Ag} & -3.770106 & 4.740886 & -1.625449\end{array}$

$\begin{array}{llll}\text { Ag } & 0.803753 & 4.738862 & 0.025871\end{array}$

$\begin{array}{llll}\mathrm{Ag} & -0.952823 & 4.672935 & -2.298770\end{array}$

$\begin{array}{llll}\text { Ag } & -4.804944 & 3.838465 & 0.811622\end{array}$

$\begin{array}{llll}\mathrm{Ag} & -1.067133 & 1.435595 & 1.399875\end{array}$

$\begin{array}{llll}\mathrm{Ag} & 1.010432 & -0.000058 & 2.752176\end{array}$

$\begin{array}{llll}\mathrm{Ag} & 3.331102 & -0.000065 & 1.067696\end{array}$

$\begin{array}{llll}\mathrm{Ag} & 1.642993 & 2.331581 & 1.185507\end{array}$

$\begin{array}{llll}\mathrm{Ag} & 5.849879 & -0.000023 & 2.118462\end{array}$

$\begin{array}{llll}\mathrm{Ag} & 1.862021 & 2.365067 & 3.987468\end{array}$

$\begin{array}{llll}\text { Ag } & 3.581159 & 3.839695 & -0.165153\end{array}$

$\begin{array}{llll}\mathrm{Ag} & 1.200821 & -0.000193 & 5.474423\end{array}$

$\begin{array}{llll}\mathrm{Ag} & 4.227111 & 2.367068 & 2.277396\end{array}$

$\begin{array}{llll}\mathrm{Ag} & 3.579847 & -0.000137 & 3.866416\end{array}$

$\begin{array}{llll}\mathrm{Ag} & -0.254802 & 3.823523 & 2.620854\end{array}$

$\begin{array}{llll}\mathrm{Ag} & -0.911635 & 1.456968 & 4.193104\end{array}$

$\begin{array}{llll}\mathrm{Ag} & 2.479472 & 4.647396 & 2.357904\end{array}$

$\begin{array}{lllll}\mathrm{Ag} & 5.298108 & 1.467864 & -0.283979\end{array}$

$\begin{array}{llll}\mathrm{Ag} & -1.972438 & 3.866763 & 0.267905\end{array}$

$\begin{array}{llll}\text { Ag } & -3.028096 & 2.862167 & 2.762733\end{array}$

$\begin{array}{llll}\text { Ag } & -5.452714 & 1.427982 & 2.219052\end{array}$

$\begin{array}{llll}\text { Ag } & -5.998019 & 0.000041 & -0.142312\end{array}$

$\begin{array}{llll}\mathrm{Ag} & -3.028066 & -2.862308 & 2.762632\end{array}$

$\begin{array}{llll}\mathrm{Ag} & -3.071450 & -0.000030 & 2.802236\end{array}$

$\begin{array}{llll}\mathrm{Ag} & -5.452729 & -1.428114 & 2.218990\end{array}$

$\begin{array}{llll}\mathrm{Ag} & -1.972337 & -3.866749 & 0.267747\end{array}$

$\begin{array}{llll}\text { Ag } & 3.581208 & -3.839643 & -0.165375\end{array}$

$\begin{array}{llll}\mathrm{Ag} & 2.479520 & -4.647528 & 2.357642\end{array}$

$\begin{array}{llll}\mathrm{Ag} & -0.254743 & -3.823627 & 2.620580\end{array}$

$\begin{array}{llll}\mathrm{Ag} & 4.227060 & -2.367157 & 2.277200\end{array}$

$\begin{array}{llll}\text { Ag } & 1.643054 & -2.331614 & 1.185390\end{array}$ 


$\begin{array}{lrrr}\mathrm{Ag} & -0.056763 & -2.344112 & -1.155886 \\ \mathrm{Ag} & 2.695747 & -1.450011 & -1.337415 \\ \mathrm{Ag} & 2.591610 & 1.478349 & -4.143315 \\ \mathrm{Ag} & 0.799686 & 0.000000 & 0.005092 \\ \mathrm{Ag} & 2.591623 & -1.478089 & -4.143350 \\ \mathrm{Ag} & -0.952823 & -4.672817 & -2.298990 \\ \mathrm{Ag} & 0.672398 & 0.000085 & -2.744416 \\ \mathrm{Ag} & 1.865587 & -3.842973 & -2.534335 \\ \mathrm{Ag} & 0.536123 & 0.000177 & -5.470385 \\ \mathrm{Ag} & -0.176052 & -2.376344 & -3.963964 \\ \mathrm{Ag} & 4.588335 & -2.887678 & -2.672329 \\ \mathrm{Ag} & 0.803782 & -4.738816 & 0.025604 \\ \mathrm{Ag} & 5.298212 & -1.467844 & -0.284141 \\ \mathrm{Ag} & 2.695722 & 1.450100 & -1.337393 \\ \mathrm{Ag} & 4.659917 & 0.000083 & -2.720115 \\ \mathrm{Ag} & -3.566285 & -1.435792 & 0.223374 \\ \mathrm{Ag} & -1.866160 & 0.000098 & -3.968273 \\ \mathrm{Ag} & -1.664252 & -0.000047 & -1.173035 \\ \mathrm{Ag} & -2.622085 & -2.361645 & -2.380966 \\ \mathrm{Ag} & -4.237970 & 0.000059 & -2.312349 \\ \mathrm{Ag} & -0.176077 & 2.376488 & -3.963836 \\ \mathrm{Ag} & -3.770123 & -4.740920 & -1.625555 \\ \mathrm{Ag} & -4.804885 & -3.838555 & 0.811620 \\ \mathrm{Ag} & -5.401380 & -2.460491 & -1.548183\end{array}$

Ag65

$\mathrm{E}=-9477.94996168$ Hartree

$\begin{array}{llll}\mathrm{Ag} & -0.707707 & -0.004100 & -5.466538\end{array}$

$\begin{array}{llll}\mathrm{Ag} & -0.809846 & -0.020151 & -2.738565\end{array}$

$\begin{array}{llll}\mathrm{Ag} & 0.199535 & -2.321821 & -3.980879\end{array}$

$\begin{array}{llll}\mathrm{Ag} & 1.166492 & -4.556042 & -2.333456\end{array}$

$\begin{array}{llll}\mathrm{Ag} & -1.704974 & -3.948483 & -2.530483\end{array}$

$\begin{array}{llll}\mathrm{Ag} & -2.627929 & -1.640989 & -4.120317\end{array}$

$\begin{array}{llll}\mathrm{Ag} & -4.491779 & -3.208247 & -2.630718\end{array}$

$\begin{array}{llll}\mathrm{Ag} & -5.281467 & -1.855288 & -0.229943\end{array}$

$\begin{array}{llll}\mathrm{Ag} & -0.171247 & 2.418279 & -3.965168\end{array}$

$\begin{array}{llll}\mathrm{Ag} & -2.859762 & 1.301658 & -4.112824\end{array}$

$\begin{array}{llll}\mathrm{Ag} & -2.930702 & 1.260949 & -1.307319\end{array}$

$\begin{array}{llll}\mathrm{Ag} & -4.792051 & -0.336682 & -2.670921\end{array}$

$\begin{array}{llll}\mathrm{Ag} & -1.924160 & 2.220125 & 1.204782\end{array}$

$\begin{array}{lllll}\mathrm{Ag} & -5.513895 & 1.069846 & -0.223357\end{array}$

$\begin{array}{llll}\mathrm{Ag} & -2.308027 & 3.713616 & -2.508188\end{array}$

$\begin{array}{lllll}\mathrm{Ag} & -4.947388 & 2.545627 & -2.617187\end{array}$

$\begin{array}{llll}\mathrm{Ag} & -3.992083 & 3.570553 & -0.120022\end{array}$

$\begin{array}{llll}\mathrm{Ag} & -2.930829 & 4.460013 & 2.391833\end{array}$

$\begin{array}{llll}\mathrm{Ag} & -2.702325 & -1.627250 & -1.314624\end{array}$ 


\begin{tabular}{|c|c|c|c|}
\hline $\mathrm{Ag}$ & -3.378072 & -4.081161 & -0.137743 \\
\hline $\mathrm{Ag}$ & -0.538137 & -4.761270 & 0.015728 \\
\hline & -1.546096 & -2.426351 & 1.192935 \\
\hline $\mathrm{Ag}$ & -1.730428 & -2.484286 & 3.996844 \\
\hline & -2.182662 & -4.804133 & 2.368676 \\
\hline & -0.908093 & -0.034651 & 0.009653 \\
\hline$g$ & -5.917320 & -0.441776 & 2.182753 \\
\hline & -4.489629 & 2.047240 & 2.327104 \\
\hline $\mathrm{g}$ & -3.631953 & -0.263585 & 3.901827 \\
\hline & -3.419240 & -0.238416 & 1.101647 \\
\hline $\mathrm{Ag}$ & -1.082420 & -0.057049 & 2.756386 \\
\hline g & -1.242429 & -0.076883 & 5.481059 \\
\hline $\mathrm{Ag}$ & -2.111908 & 2.230482 & 4.008025 \\
\hline$g$ & -4.107553 & -2.671329 & 2.315065 \\
\hline $\mathrm{Ag}$ & 0.434520 & 4.765564 & -2.304893 \\
\hline $\mathrm{Ag}$ & 2.282622 & 2.593378 & -2.401276 \\
\hline $\mathrm{Ag}$ & -0.137847 & 3.862050 & 2.621797 \\
\hline $\mathrm{Ag}$ & -0.255245 & 2.368936 & -1.156119 \\
\hline $\mathrm{Ag}$ & 1.543925 & 0.152820 & -1.181547 \\
\hline $\mathrm{Ag}$ & 3.333836 & 1.742911 & \\
\hline $\mathrm{Ag}$ & -1.293133 & 4.686918 & 0.040198 \\
\hline $\mathrm{Ag}$ & 1.546171 & 4.04 & 0.2 \\
\hline $\mathrm{Ag}$ & 4.372542 & 4.245218 & 0.759589 \\
\hline $\mathrm{Ag}$ & 5.228809 & 1.90 & \\
\hline $\mathrm{Ag}$ & 0.849688 & 1.54 & 1.3 \\
\hline $\mathrm{Ag}$ & 0.963608 & -1.357383 & 4.1 \\
\hline $\mathrm{Ag}$ & 0.724601 & 1.55 & 4.182866 \\
\hline $\mathrm{Ag}$ & 2.702761 & 3.129967 & 2.731831 \\
\hline $\mathrm{Ag}$ & 5.050544 & 2.923677 & -1.608517 \\
\hline $\mathrm{Ag}$ & 3.241676 & 5.063596 & -1.666448 \\
\hline $\mathrm{Ag}$ & 0.106968 & -2.304354 & -1.171327 \\
\hline $\mathrm{Ag}$ & 5.007093 & -3.447641 & 0.730772 \\
\hline $\mathrm{Ag}$ & 2.164251 & -3.662050 & 0.217117 \\
\hline $\mathrm{Ag}$ & 3.980332 & -4.422812 & -1.702919 \\
\hline $\mathrm{Ag}$ & 2.646645 & -2.119132 & -2.419358 \\
\hline $\mathrm{Ag}$ & 1.702738 & 0.182735 & -3.984907 \\
\hline $\mathrm{Ag}$ & 4.085593 & 0.374918 & -2.358296 \\
\hline $\mathrm{Ag}$ & 5.436029 & -2.023069 & -1.715785 \\
\hline $\mathrm{Ag}$ & 3.614578 & -1.125827 & 0.176523 \\
\hline $\mathrm{Ag}$ & 3.164728 & -2.592166 & 2.708653 \\
\hline $\mathrm{Ag}$ & 5.889975 & 0.519206 & -0.207222 \\
\hline $\mathrm{Ag}$ & 7.219139 & -1.845477 & 0.348108 \\
\hline $\mathrm{Ag}$ & 2.969361 & 0.279788 & 2.767053 \\
\hline $\mathrm{Ag}$ & 0.481189 & -3.761599 & 2.593525 \\
\hline $\mathrm{Ag}$ & 1.099352 & -1.313104 & 1.363979 \\
\hline $\mathrm{Ag}$ & 5.459035 & -0.945219 & 2.235318 \\
\hline
\end{tabular}




$\begin{array}{lrrr}\mathrm{Ag} 66 & & & \\ \mathrm{E}=-9623.75463037 & \text { Hartree } & \\ \mathrm{Ag} & -2.171936 & 4.189233 & 1.859091 \\ \mathrm{Ag} & 0.637936 & 4.616612 & 1.102746 \\ \mathrm{Ag} & -5.168748 & 2.690719 & -1.745782 \\ \mathrm{Ag} & 2.150028 & 2.450306 & 0.152916 \\ \mathrm{Ag} & 1.414342 & 4.382337 & -1.743501 \\ \mathrm{Ag} & -3.315752 & 4.495015 & -2.819163 \\ \mathrm{Ag} & -3.210318 & 2.085255 & 0.076689 \\ \mathrm{Ag} & -4.083172 & 4.697324 & -0.132877 \\ \mathrm{Ag} & -1.368391 & 4.077954 & -0.924297 \\ \mathrm{Ag} & 2.757685 & 3.449534 & 2.707574 \\ \mathrm{Ag} & 6.191326 & 0.036025 & 1.489980 \\ \mathrm{Ag} & 3.375346 & 4.882067 & 0.289781 \\ \mathrm{Ag} & 4.385591 & -1.854245 & 2.681689 \\ \mathrm{Ag} & 4.852433 & 2.502792 & 0.902218 \\ \mathrm{Ag} & 3.563643 & 0.017826 & 0.757502 \\ \mathrm{Ag} & 4.189846 & 0.984011 & 3.318182 \\ \mathrm{Ag} & 1.501011 & 0.951034 & 2.529529 \\ \mathrm{Ag} & -0.581778 & 2.097179 & 0.943503 \\ \mathrm{Ag} & 2.062811 & 1.896745 & 5.025668 \\ \mathrm{Ag} & -0.039946 & 3.091135 & 3.499881 \\ \mathrm{Ag} & -4.366121 & -0.014518 & -1.675558 \\ \mathrm{Ag} & 2.276534 & -0.904396 & 4.482348 \\ \mathrm{Ag} & -0.349604 & 0.389998 & 4.561872 \\ \mathrm{Ag} & 1.693953 & -1.842162 & 1.905398 \\ \mathrm{Ag} & -4.790850 & 3.022144 & 2.104912 \\ \mathrm{Ag} & -0.151022 & -2.440964 & 3.934591 \\ \mathrm{Ag} & -0.886848 & -0.570115 & 1.986970 \\ \mathrm{Ag} & -5.817445 & 0.955866 & 0.500618 \\ \mathrm{Ag} & -2.791705 & -1.122543 & 3.935785 \\ \mathrm{Ag} & -5.101665 & 0.381304 & 3.171061 \\ \mathrm{Ag} & -2.513522 & 1.561109 & 2.953422 \\ \mathrm{Ag} & -3.522839 & -0.543830 & 1.146918 \\ \mathrm{Ag} & -4.888356 & -2.458155 & 2.555145 \\ \mathrm{Ag} & 2.450255 & -3.673537 & 3.780084 \\ \mathrm{Ag} & -0.259731 & -2.478288 & -0.086462 \\ \mathrm{Ag} & -0.649318 & 3.747104 & -3.697662 \\ \mathrm{Ag} & -2.341370 & -1.108867 & -3.379382 \\ \mathrm{Ag} & -0.371780 & -1.956544 & -4.989286 \\ \mathrm{Ag} & -3.535434 & -2.654157 \\ \mathrm{Ag} & -0.004313 & -0.847376 \\ \mathrm{Ag} & -1.000884 & -2.494987 \\ \mathrm{Ag} & -2.763660\end{array}$




$\begin{array}{lrrr}\mathrm{Ag} & -3.427949 & -4.808291 & -2.182185 \\ \mathrm{Ag} & -4.294729 & -4.366610 & 0.501890 \\ \mathrm{Ag} & -2.185110 & -3.096433 & 1.930274 \\ \mathrm{Ag} & -5.629264 & -1.948749 & -0.103710 \\ \mathrm{Ag} & -4.827697 & -2.523517 & -2.797931 \\ \mathrm{Ag} & -2.909245 & -2.454999 & -0.857606 \\ \mathrm{Ag} & 1.263816 & -4.629311 & -0.989777 \\ \mathrm{Ag} & -1.532371 & -4.947101 & -0.168274 \\ \mathrm{Ag} & 3.266462 & -3.995129 & 1.039334 \\ \mathrm{Ag} & 5.174134 & -2.102810 & -0.124903 \\ \mathrm{Ag} & 0.923230 & -0.000206 & 0.013651 \\ \mathrm{Ag} & 0.481232 & -4.373365 & 1.831002 \\ \mathrm{Ag} & 2.730880 & 0.569387 & -1.952575 \\ \mathrm{Ag} & 1.988107 & 2.465291 & -3.881321 \\ \mathrm{Ag} & 4.011754 & 3.063952 & -1.847637 \\ \mathrm{Ag} & 2.470428 & -2.091545 & -0.860193 \\ \mathrm{Ag} & 4.544200 & 1.136681 & -3.904489 \\ \mathrm{Ag} & 5.446592 & 0.594094 & -1.231934 \\ \mathrm{Ag} & 4.002790 & -4.169976 & -1.731995 \\ \mathrm{Ag} & 2.214845 & -0.410037 & -4.523797 \\ \mathrm{Ag} & 4.334670 & -1.545399 & -2.874176 \\ \mathrm{Ag} & 1.931948 & -3.126920 & -3.412643 \\ \mathrm{Ag} & 0.160790 & 1.881847 & -1.855256\end{array}$

\begin{tabular}{|c|c|c|c|}
\hline \multicolumn{4}{|c|}{ Ag67 } \\
\hline \multicolumn{4}{|c|}{$E=-9769.57496686$ Hartree } \\
\hline $\mathrm{Ag}$ & -1.377370 & 0.872422 & 1.216346 \\
\hline $\mathrm{Ag}$ & -3.765631 & 1.772327 & 2.393958 \\
\hline $\mathrm{Ag}$ & -5.621953 & -0.348600 & 1.904116 \\
\hline $\mathrm{Ag}$ & -4.158726 & & \\
\hline $\mathrm{Ag}$ & -2.812676 & -2.691989 & 0.297930 \\
\hline $\mathrm{Ag}$ & -4.738703 & -3.056532 & \\
\hline $\mathrm{Ag}$ & -1.377478 & -0.872324 & -1.216391 \\
\hline $\mathrm{Ag}$ & -2.8123 & 2.6923 & -0.2 \\
\hline $\mathrm{Ag}$ & -5.5 & -2.1 & \\
\hline $\mathrm{Ag}$ & -3.7 & 0.0 & 024 \\
\hline $\mathrm{Ag}$ & -5.5 & 2.1 & 784 \\
\hline $\mathrm{Ag}$ & -2.0 & 3.6 & 717 \\
\hline $\mathrm{Ag}$ & -1.908973 & 6.0 & 707 \\
\hline $\mathrm{Ag}$ & -5.621922 & 0.3 & -1.9 \\
\hline $\mathrm{Ag}$ & -3.303779 & 5.281579 & -1.131016 \\
\hline $\mathrm{Ag}$ & -4.738288 & 3.057059 & -2.219553 \\
\hline $\mathrm{Ag}$ & -0.561488 & 4.498695 & -0.670220 \\
\hline $\mathrm{Ag}$ & -2.940921 & 0.940395 & -2.725014 \\
\hline $\mathrm{Ag}$ & 0.936070 & & -3.038682 \\
\hline $\mathrm{Ag}$ & -0.500999 & 1.805200 & -1.524675 \\
\hline
\end{tabular}




\begin{tabular}{|c|c|c|c|}
\hline g & -0.496747 & 1.475199 & -4.300513 \\
\hline $\mathrm{Ag}$ & -3.765843 & -1.771904 & -2.393933 \\
\hline & 2.359373 & 4.465931 & -0.598248 \\
\hline & 0.868143 & 2.605672 & 0.883696 \\
\hline & 0.793710 & 5.205027 & 1.756770 \\
\hline & 3.241121 & 3.574067 & 2.018773 \\
\hline $\mathrm{Ag}$ & 0.934240 & 2.997158 & 3.670626 \\
\hline & 3.316726 & 1.312263 & 3.892179 \\
\hline $\mathrm{Ag}$ & -0.496931 & -1.475297 & 4.300522 \\
\hline & 0.955999 & 0.361409 & 2.736559 \\
\hline Ag & -2.941027 & -0.940069 & 2.725057 \\
\hline $\mathrm{Ag}$ & -1.496456 & 3.531222 & 2.156906 \\
\hline $\mathrm{Ag}$ & -1.401289 & 1.254267 & 3.992556 \\
\hline $\mathrm{Ag}$ & 0.987753 & 0.716902 & 5.443718 \\
\hline $\mathrm{Ag}$ & -2.013957 & -3.617381 & 2.998614 \\
\hline Ag & -3.304425 & 1198 & 1.131048 \\
\hline $\mathrm{Ag}$ & -1.496879 & -3.531109 & -2.156794 \\
\hline $\mathrm{Ag}$ & -4.159291 & -4.390283 & -1.385026 \\
\hline Ag & -1.401408 & -1.254190 & -3.992543 \\
\hline $\mathrm{Ag}$ & 2.355416 & -1.8 & 1.474600 \\
\hline $\mathrm{Ag}$ & 3.799148 & -3.600234 & 2.937023 \\
\hline $\mathrm{Ag}$ & 4.747535 & -0.8 & 2.616874 \\
\hline $\mathrm{Ag}$ & 2.358912 & -4.46 & 98242 \\
\hline $\mathrm{Ag}$ & 3.232435 & 0.926010 & 1.108711 \\
\hline $\mathrm{Ag}$ & 5.554075 & 1.84 & 2.206743 \\
\hline $\mathrm{Ag}$ & 0.935621 & -3.665887 & 3.038622 \\
\hline $\mathrm{Ag}$ & 2.421429 & -1.467198 & 4.265524 \\
\hline $\mathrm{Ag}$ & 0.905414 & -0.000045 & 0.000021 \\
\hline Ag & 0.867761 & -2.605774 & -0.883768 \\
\hline $\mathrm{Ag}$ & 5.637079 & -0.000222 & 0.000058 \\
\hline $\mathrm{Ag}$ & 4.741198 & 2.772033 & -0.367386 \\
\hline $\mathrm{Ag}$ & 3.232311 & -0.926249 & -1.108748 \\
\hline $\mathrm{Ag}$ & 4.740905 & -2.772410 & 0.367334 \\
\hline $\mathrm{Ag}$ & 0.933847 & -2.997402 & -3.670649 \\
\hline $\mathrm{Ag}$ & 5.553916 & -1.842527 & -2.206777 \\
\hline $\mathrm{Ag}$ & 0.793140 & -5.205241 & -1.756740 \\
\hline $\mathrm{Ag}$ & 3.240768 & -3.574324 & -2.018781 \\
\hline $\mathrm{Ag}$ & 3.316575 & -1.312579 & -3.892116 \\
\hline $\mathrm{Ag}$ & 0.955966 & -0.361618 & -2.736607 \\
\hline $\mathrm{Ag}$ & 2.355571 & 1.806854 & -1.474592 \\
\hline $\mathrm{Ag}$ & 0.987641 & -0.717161 & -5.443677 \\
\hline $\mathrm{Ag}$ & 2.421581 & 1.466932 & -4.265492 \\
\hline $\mathrm{Ag}$ & 3.799470 & 3.599860 & -2.937122 \\
\hline $\mathrm{Ag}$ & 4.747759 & 0.895022 & -2.616730 \\
\hline $\mathrm{Ag}$ & -0.501237 & -1.805226 & 1.524579 \\
\hline $\mathrm{Ag}$ & -0.561987 & -4.498691 & 0.670171 \\
\hline
\end{tabular}




$$
\begin{array}{llll}
\text { Ag } & -1.909754 & -6.055597 & -1.160664
\end{array}
$$

$\begin{array}{lrrr}\mathrm{Ag} 68 & & & \\ \mathrm{E}=-9915.39042084 \text { Hartree } & \\ \mathrm{Ag} & -5.659519 & -1.823440 & 2.224220 \\ \mathrm{Ag} & -3.337881 & -0.915712 & 1.116874 \\ \mathrm{Ag} & -4.850103 & 2.773738 & 0.341860 \\ \mathrm{Ag} & -2.468249 & 4.467252 & 0.558048 \\ \mathrm{Ag} & -0.972009 & 2.593384 & -0.907062 \\ \mathrm{Ag} & -0.903069 & 5.187185 & -1.803409 \\ \mathrm{Ag} & 1.792359 & 6.051381 & -1.217532 \\ \mathrm{Ag} & -3.345976 & 3.555060 & -2.050406 \\ \mathrm{Ag} & 4.043418 & 4.385263 & -1.409499 \\ \mathrm{Ag} & 1.391597 & 3.505987 & -2.170041 \\ \mathrm{Ag} & 2.712988 & 2.687662 & 0.293017 \\ \mathrm{Ag} & -1.008672 & 0.000037 & -0.000029 \\ \mathrm{Ag} & -0.971947 & -2.593479 & 0.907021 \\ \mathrm{Ag} & -1.054201 & -0.339324 & 2.735703 \\ \mathrm{Ag} & 4.611216 & 3.112968 & 2.226687 \\ \mathrm{Ag} & -2.465522 & 1.817430 & 1.458646 \\ \mathrm{Ag} & 1.293138 & -0.848263 & 1.204065 \\ \mathrm{Ag} & -3.418110 & -1.279488 & 3.901490 \\ \mathrm{Ag} & 1.391744 & -3.505903 & 2.170020 \\ \mathrm{Ag} & -1.036058 & -2.967278 & 3.695864 \\ \mathrm{Ag} & -2.530952 & 1.498894 & 4.250202 \\ \mathrm{Ag} & 0.386137 & 1.824887 & 1.512510 \\ \mathrm{Ag} & -3.912734 & 3.622288 & 2.904880 \\ \mathrm{Ag} & -4.857897 & 0.915922 & 2.609103 \\ \mathrm{Ag} & 0.450667 & 4.504628 & 0.629136 \\ \mathrm{Ag} & 1.887108 & 3.656650 & 2.977080 \\ \mathrm{Ag} & 3.176922 & 5.301446 & 1.089194 \\ \mathrm{Ag} & -1.053617 & 3.696018 & 3.009021 \\ \mathrm{Ag} & 0.379864 & 1.516937 & 4.293923 \\ \mathrm{Ag} & 5.491923 & 0.405517 & 2.042169 \\ \mathrm{Ag} & 1.298889 & -1.220404 & 3.989795 \\ \mathrm{Ag} & 2.809658 & 0.974186 & 2.730779 \\ \mathrm{Ag} & 3.665454 & -1.755556 & 2.390774 \\ \mathrm{Ag} & -1.089239 & -0.673650 & 5.446495 \\ \mathrm{Ag} & -5.7437916 & 0.915597 & -1.116897 \\ \mathrm{Ag} & -0.000053 & -0.000003 \\ \mathrm{Ag} & -3.6548 & 1.823398 & -2.224198 \\ \mathrm{Ag} & 0.339317 & -2.735681 \\ \mathrm{Ag} & -3.622438 & -2.904835 \\ \mathrm{Ag} & -1.817486 & -1.458671 \\ \mathrm{Ag} & -367302 & -0.558023 \\ \mathrm{Ag} & -3.009003\end{array}$




$\begin{array}{lrrr}\mathrm{Ag} & -4.857896 & -0.915971 & -2.608986 \\ \mathrm{Ag} & -4.850104 & -2.773871 & -0.341830 \\ \mathrm{Ag} & -3.345892 & -3.555242 & 2.050433 \\ \mathrm{Ag} & -1.089329 & 0.673652 & -5.446424 \\ \mathrm{Ag} & -2.530950 & -1.498902 & -4.250210 \\ \mathrm{Ag} & 1.298802 & 1.220429 & -3.989804 \\ \mathrm{Ag} & -1.036230 & 2.967204 & -3.695797 \\ \mathrm{Ag} & -3.418238 & 1.279392 & -3.901383 \\ \mathrm{Ag} & 0.379909 & -1.516901 & -4.293919 \\ \mathrm{Ag} & 3.665357 & 1.755592 & -2.390807 \\ \mathrm{Ag} & 2.809570 & -0.974125 & -2.730782 \\ \mathrm{Ag} & 0.386162 & -1.824856 & -1.512499 \\ \mathrm{Ag} & 1.293072 & 0.848259 & -1.204154 \\ \mathrm{Ag} & 1.887128 & -3.656620 & -2.977049 \\ \mathrm{Ag} & 1.792509 & -6.051317 & 1.217574 \\ \mathrm{Ag} & 3.677690 & 0.000095 & -0.000012 \\ \mathrm{Ag} & 3.177129 & -5.301377 & -1.089227 \\ \mathrm{Ag} & 7.238342 & 0.000096 & -0.000024 \\ \mathrm{Ag} & 4.611254 & -3.112918 & -2.226775 \\ \mathrm{Ag} & 5.491859 & -0.405387 & -2.042182 \\ \mathrm{Ag} & 5.481634 & -2.129774 & 0.280399 \\ \mathrm{Ag} & -0.902903 & -5.187287 & 1.803328 \\ \mathrm{Ag} & 0.450745 & -4.504567 & -0.629150 \\ \mathrm{Ag} & 4.043551 & -4.385136 & 1.409453 \\ \mathrm{Ag} & 2.713125 & -2.687568 & -0.293014 \\ \mathrm{Ag} & 5.481579 & 2.129878 & -0.280444\end{array}$

Ag69

$\mathrm{E}=-10061.1874990$ Hartree

$\begin{array}{lrrr}\mathrm{Ag} & -0.928238 & 1.780265 & -4.335496 \\ \mathrm{Ag} & 1.707558 & 0.624738 & -1.485440 \\ \mathrm{Ag} & 3.558705 & -1.016448 & -2.905109 \\ \mathrm{Ag} & 5.650553 & 0.214549 & -1.408630 \\ \mathrm{Ag} & 3.214232 & -3.847646 & -2.812326 \\ \mathrm{Ag} & 5.321097 & -2.617888 & -1.402684 \\ \mathrm{Ag} & -0.421307 & -0.630510 & -5.732689 \\ \mathrm{Ag} & 1.404493 & -2.258897 & -4.310398 \\ \mathrm{Ag} & 1.410290 & -2.243668 & -1.443370 \\ \mathrm{Ag} & -2.841892 & -0.371842 & -1.445292 \\ \mathrm{Ag} & -3.835219 & -2.620272 & -2.839457 \\ \mathrm{Ag} & -5.242145 & -0.134869 & -2.816164 \\ \mathrm{Ag} & 0.430404 & -4.535702 & 0.000043 \\ \mathrm{Ag} & 3.224762 & -3.909491 & -0.000014 \\ \mathrm{Ag} & -2.348017 & -5.025489 & -2.773457 \\ \mathrm{Ag} & -1.381060 & -2.825628 & -1.426187 \\ \mathrm{Ag} & 0.429695 & -4.495939 & -2.839273\end{array}$




\begin{tabular}{|c|c|c|c|}
\hline g & -2.852137 & & \\
\hline & & & \\
\hline & -0.412563 & & \\
\hline & & & \\
\hline & 0969 & & \\
\hline & & & \\
\hline & & & \\
\hline & & & \\
\hline & & & \\
\hline & 1.1 & & \\
\hline & & & \\
\hline & 5.8 & & \\
\hline & & & \\
\hline & -1.4 & & \\
\hline & & & \\
\hline & -3.8 & & \\
\hline & 3.7 & & -0.0 \\
\hline & & & \\
\hline & 1.4 & -2.2 & \\
\hline & & & \\
\hline & 3.5 & -1.0 & \\
\hline & -2.3 & & \\
\hline & -0.4 & -0.6 & \\
\hline & & & \\
\hline & -1.3 & & \\
\hline & -0.4 & -0.6 & \\
\hline & & -2.6 & \\
\hline & 1.4 & -2.2 & \\
\hline & & -3.8 & \\
\hline & 0.4 & -4.4 & \\
\hline & -2.3 & -5.0 & \\
\hline & -5.2 & -0.1 & $-0 .($ \\
\hline & & & \\
\hline & & & \\
\hline & -5.7 & & \\
\hline & -1.4 & & \\
\hline & -0.9 & & \\
\hline & -3.89 & 4.3596 & \\
\hline & -2.8 & -0.3 & \\
\hline & -3.3 & & \\
\hline & -2.8 & -0.38 & \\
\hline & & & \\
\hline & -3.350195 & 2.005394 & -0.000002 \\
\hline & -5.755439 & & -1.402353 \\
\hline & 1.208362 & 3.043581 & 2.914524 \\
\hline & 1.707576 & 0.624759 & 1.485388 \\
\hline
\end{tabular}




$$
\begin{array}{lrrc}
\mathrm{Ag} & -1.452174 & 4.221983 & -0.000009 \\
\mathrm{Ag} & 5.650545 & 0.214509 & 1.408586 \\
\mathrm{Ag} & 3.831069 & 1.848763 & 2.879965 \\
\mathrm{Ag} & 5.873638 & 3.028259 & 1.371921 \\
\mathrm{Ag} & 3.517653 & -1.021971 & -0.000042 \\
\mathrm{Ag} & 3.321920 & 4.235842 & 1.409197
\end{array}
$$

\begin{tabular}{lrrr}
$\mathrm{Ag} 70$ & \multicolumn{3}{c}{} \\
$\mathrm{E}=-10206.9996136$ & & \\
$\mathrm{Ag}$ & 1.463390 & -4.429205 & 1.461640 \\
$\mathrm{Ag}$ & -0.036801 & -2.470465 & 0.163381 \\
$\mathrm{Ag}$ & 0.328404 & -3.030484 & -3.821458 \\
$\mathrm{Ag}$ & 0.125982 & -0.304711 & -4.877129 \\
$\mathrm{Ag}$ & -0.543578 & -0.907494 & -2.238816 \\
$\mathrm{Ag}$ & -1.814294 & -3.431384 & -1.849845 \\
$\mathrm{Ag}$ & -2.316735 & -1.794810 & -4.189781 \\
$\mathrm{Ag}$ & -4.477133 & -3.008198 & -2.765082 \\
$\mathrm{Ag}$ & -0.362122 & 1.910256 & -1.533338 \\
$\mathrm{Ag}$ & -3.260601 & -0.866968 & -1.613188 \\
$\mathrm{Ag}$ & -2.145911 & 1.034456 & -3.579496 \\
$\mathrm{Ag}$ & -4.502929 & 2.570081 & -3.139741 \\
$\mathrm{Ag}$ & -4.683318 & -0.230604 & -3.840900 \\
$\mathrm{Ag}$ & -5.413003 & -2.154207 & -0.259619 \\
$\mathrm{Ag}$ & -3.973348 & -4.561736 & -0.377369 \\
$\mathrm{Ag}$ & -2.761203 & -2.406842 & 0.753565 \\
$\mathrm{Ag}$ & -1.307483 & -4.907260 & 0.593768 \\
$\mathrm{Ag}$ & 0.840208 & -4.608867 & -1.395732 \\
$\mathrm{Ag}$ & 1.832192 & 0.590885 & -2.831354 \\
$\mathrm{Ag}$ & 2.524539 & 1.188066 & -5.394630 \\
$\mathrm{Ag}$ & 0.309301 & 2.540635 & -4.164732 \\
$\mathrm{Ag}$ & 4.561634 & 0.572452 & -3.459800 \\
$\mathrm{Ag}$ & 2.751649 & -1.513670 & -4.435885 \\
$\mathrm{Ag}$ & 2.031578 & -2.094337 & -1.791362 \\
$\mathrm{Ag}$ & 6.471742 & -0.064541 & -1.407393 \\
$\mathrm{Ag}$ & 3.812430 & -0.038021 & -0.829083 \\
$\mathrm{Ag}$ & 0.807468 & 4.404800 & -1.959781 \\
$\mathrm{Ag}$ & 1.147736 & -0.011460 & -0.249570 \\
$\mathrm{Ag}$ & 4.763844 & -2.151353 & -2.404948 \\
$\mathrm{Ag}$ & 3.047321 & 3.073842 & -3.287214 \\
$\mathrm{Ag}$ & -1.955306 & 3.816262 & -2.785036 \\
$\mathrm{Ag}$ & 5.059315 & 2.435266 & -1.256504 \\
$\mathrm{Ag}$ & 5.386560 & -1.971887 & 0.450304 \\
$\mathrm{Ag}$ & 2.921665 & -4.160179 & -3.323343 \\
$\mathrm{Ag}$ & 2.645544 & -1.917341 & 1.023495 \\
& -0.793351 & -3.136991 & 2.831609
\end{tabular}




$\begin{array}{lrrr}\mathrm{Ag} & -1.329851 & 4.088798 & 0.032935 \\ \mathrm{Ag} & -0.722718 & 4.171613 & 2.866807 \\ \mathrm{Ag} & -0.493892 & 1.510737 & 3.995220 \\ \mathrm{Ag} & -2.860291 & 3.043782 & 4.392535 \\ \mathrm{Ag} & 0.457975 & -0.618716 & 2.353458 \\ \mathrm{Ag} & -0.322366 & -1.219707 & 4.954908 \\ \mathrm{Ag} & -2.660470 & 0.352635 & 5.434843 \\ \mathrm{Ag} & -3.380214 & 4.647165 & 1.928143 \\ \mathrm{Ag} & -2.471854 & 2.085071 & 1.878132 \\ \mathrm{Ag} & 0.256862 & 2.088685 & 1.304883 \\ \mathrm{Ag} & 1.432461 & 4.585053 & 0.906171 \\ \mathrm{Ag} & 4.502973 & 1.758343 & 3.676791 \\ \mathrm{Ag} & 4.144360 & -3.807661 & 2.283106 \\ \mathrm{Ag} & 4.382292 & -1.043658 & 3.040760 \\ \mathrm{Ag} & 1.962939 & -2.559251 & 3.673157 \\ \mathrm{Ag} & 2.145699 & 0.277434 & 4.383483 \\ \mathrm{Ag} & 5.569166 & 0.862819 & 1.160139 \\ \mathrm{Ag} & 2.825584 & 0.877195 & 1.723131 \\ \mathrm{Ag} & 2.322892 & 2.427363 & -0.659204 \\ \mathrm{Ag} & 1.942740 & 3.011420 & 3.324552 \\ \mathrm{Ag} & 4.055040 & 3.364287 & 1.333574 \\ \mathrm{Ag} & 3.501916 & 4.845944 & -1.068359 \\ \mathrm{Ag} & -2.846610 & -2.538054 & 4.711153 \\ \mathrm{Ag} & -2.273713 & -0.582444 & 2.911382 \\ \mathrm{Ag} & -4.759451 & -1.965731 & 2.737196 \\ \mathrm{Ag} & -3.338343 & -4.378651 & 2.534234 \\ \mathrm{Ag} & -4.256150 & 0.042437 & 0.925534 \\ \mathrm{Ag} & -4.567737 & 1.009472 & 3.482053 \\ \mathrm{Ag} & -1.476194 & 0.014705 & 0.320966 \\ \mathrm{Ag} & -3.081692 & 1.909226 & -0.918163 \\ \mathrm{Ag} & -5.102915 & 2.659827 & 0.945607 \\ \mathrm{Ag} & -5.625259 & 0.704550 & -1.366873 \\ \mathrm{Ag} & -3.995408 & 4.469770 & -0.892916\end{array}$

Ag71

$\mathrm{E}=-10352.8469247$ Hartree

$\begin{array}{llll}\mathrm{Ag} & -2.572897 & 1.866202 & -5.951069\end{array}$

$\begin{array}{llll}\mathrm{Ag} & -3.388004 & -2.276978 & 2.029519\end{array}$

$\begin{array}{llll}\mathrm{Ag} & -5.680789 & -1.051356 & -2.095898\end{array}$

Ag $\quad-4.192396 \quad 0.405486 \quad-4.076906$

$\begin{array}{llll}\mathrm{Ag} & -1.534948 & 1.113375 & -3.550310\end{array}$

$\begin{array}{llll}\mathrm{Ag} & -3.095157 & -0.352214 & -1.602953\end{array}$

$\begin{array}{llll}\mathrm{Ag} & -4.842739 & -0.308331 & 0.560129\end{array}$

$\begin{array}{llll}\text { Ag } & 0.183050 & 1.067914 & -5.760941\end{array}$

$\begin{array}{llll}\mathrm{Ag} & -1.961653 & -0.898627 & -5.450369\end{array}$

$\begin{array}{llll}\text { Ag } & 2.063313 & 2.763276 & -4.312592\end{array}$ 


$\begin{array}{lrrr}\mathrm{Ag} & -3.546563 & -2.379780 & -3.500026 \\ \mathrm{Ag} & -3.992983 & -2.881676 & -0.688682 \\ \mathrm{Ag} & -3.426470 & 3.177941 & -3.538727 \\ \mathrm{Ag} & -0.500073 & 0.362754 & -1.156711 \\ \mathrm{Ag} & -3.804846 & 0.332713 & 3.266786 \\ \mathrm{Ag} & -4.183757 & 2.464190 & 1.070222 \\ \mathrm{Ag} & -2.194009 & 0.520215 & 1.065637 \\ \mathrm{Ag} & -5.009556 & 1.693147 & -1.590282 \\ \mathrm{Ag} & -0.722295 & 3.587334 & -4.579433 \\ \mathrm{Ag} & 0.338165 & 2.779861 & -2.105213 \\ \mathrm{Ag} & 1.140564 & 5.189727 & -3.088033 \\ \mathrm{Ag} & 0.028074 & 2.395599 & 0.693102 \\ \mathrm{Ag} & 2.668538 & 3.489149 & 1.219252 \\ \mathrm{Ag} & -1.542541 & 4.871708 & -2.092445 \\ \mathrm{Ag} & -1.941856 & 4.412748 & 0.786450 \\ \mathrm{Ag} & 2.974669 & 3.520126 & -1.619156 \\ \mathrm{Ag} & 0.246673 & 1.358429 & 3.261212 \\ \mathrm{Ag} & 0.704616 & 4.914185 & -0.274967 \\ \mathrm{Ag} & 0.315428 & 4.223697 & 2.705591 \\ \mathrm{Ag} & -0.201629 & 3.124068 & 5.260602 \\ \mathrm{Ag} & -3.092119 & -1.818828 & 4.960310 \\ \mathrm{Ag} & -4.170211 & 4.392445 & -1.041950 \\ \mathrm{Ag} & -2.125972 & 2.658991 & 3.031821 \\ \mathrm{Ag} & -1.237538 & -1.052089 & 3.147003 \\ \mathrm{Ag} & -2.331599 & 2.383589 & -1.075224 \\ \mathrm{Ag} & -1.667551 & 0.692509 & 5.239541 \\ \mathrm{Ag} & 1.224733 & 0.289007 & -3.269619 \\ \mathrm{Ag} & 0.824710 & -1.718352 & -5.182549 \\ \mathrm{Ag} & 2.912316 & 0.238722 & -5.406595 \\ \mathrm{Ag} & -1.961526 & -4.529748 & 0.944946 \\ \mathrm{Ag} & 4.917154 & 2.335465 & 2.800245 \\ \mathrm{Ag} & 3.916627 & 1.082594 & -2.865186 \\ \mathrm{Ag} & 2.190007 & 0.975900 & -0.686777 \\ \mathrm{Ag} & 4.705210 & 1.766076 & 0.036951 \\ \mathrm{Ag} & 1.304014 & -1.776901 & -1.167089 \\ \mathrm{Ag} & 2.757549 & 0.899667 & 2.031816 \\ \mathrm{Ag} & 5.190152 & -3.094753 & 0.979369 \\ \mathrm{Ag} & 3.297991 & -3.643915 & -1.051142 \\ \mathrm{Ag} & 5.177506 & -0.419806 & 1.931250 \\ \mathrm{Ag} & 4.369697 & -0.933770 & -0.903221 \\ \mathrm{Ag} & -1.3033878 & -1.646711 & -2.959233 \\ \mathrm{Ag} & -3.735545 & -3.120957 \\ \mathrm{Ag} & -1.752619 & -3.332153 \\ \mathrm{Ag} & -3.618501 & -4.793433 \\ \mathrm{Ag} & & -118813 & -1.985224\end{array}$




$\begin{array}{lrrr}\mathrm{Ag} & -1.474781 & -3.810481 & 3.744107 \\ \mathrm{Ag} & 2.443834 & 2.788019 & 4.119188 \\ \mathrm{Ag} & 0.356116 & -3.000657 & 1.846959 \\ \mathrm{Ag} & -0.262222 & -1.882447 & 5.610300 \\ \mathrm{Ag} & 1.437115 & -3.862775 & 4.254509 \\ \mathrm{Ag} & 2.596244 & -1.883160 & 6.004941 \\ \mathrm{Ag} & 3.852650 & 0.229621 & 4.493575 \\ \mathrm{Ag} & 1.564435 & -1.134709 & 3.618366 \\ \mathrm{Ag} & 1.230652 & 0.646848 & 5.758003 \\ \mathrm{Ag} & 0.498875 & -0.361802 & 1.153878 \\ \mathrm{Ag} & 2.825162 & -1.794393 & 1.157730 \\ \mathrm{Ag} & 3.980220 & -2.557513 & 3.564362 \\ \mathrm{Ag} & 0.240166 & -5.662206 & 2.314348 \\ \mathrm{Ag} & 2.755678 & -4.497886 & 1.699440 \\ \mathrm{Ag} & 0.626508 & -4.497420 & -0.402460\end{array}$

Ag72

$\mathrm{E}=-10498.6329234$ Hartree

$\begin{array}{lrrr}\mathrm{Ag} & 2.423944 & 2.666411 & 0.145349 \\ \mathrm{Ag} & -0.083553 & 2.012784 & 4.388973 \\ \mathrm{Ag} & -2.536588 & 0.494578 & 4.282083 \\ \mathrm{Ag} & -0.229995 & -0.398101 & 2.920151 \\ \mathrm{Ag} & -2.355268 & 0.614222 & -1.514799 \\ \mathrm{Ag} & -2.451709 & 0.559431 & 1.415153 \\ \mathrm{Ag} & -2.320516 & 2.990538 & 2.916012 \\ \mathrm{Ag} & -2.089428 & 5.394614 & 1.461489 \\ \mathrm{Ag} & -2.200164 & 3.007972 & 0.002042 \\ \mathrm{Ag} & -4.385908 & 3.967866 & -1.461320 \\ \mathrm{Ag} & -4.694145 & 1.511122 & -0.107699 \\ \mathrm{Ag} & -4.477994 & 3.914978 & 1.354359 \\ \mathrm{Ag} & -1.997490 & 5.447785 & -1.355671 \\ \mathrm{Ag} & -2.129384 & 3.100033 & -2.911406 \\ \mathrm{Ag} & -4.562918 & 1.550667 & -2.941107 \\ \mathrm{Ag} & -4.082595 & -1.358430 & 2.767779 \\ \mathrm{Ag} & -3.945284 & -1.297831 & -0.135668 \\ \mathrm{Ag} & -5.550531 & -3.172198 & 1.180005 \\ \mathrm{Ag} & -4.749146 & 1.444324 & 2.727854 \\ \mathrm{Ag} & -6.152663 & -0.389025 & -1.600022 \\ \mathrm{Ag} & -6.244833 & -0.442017 & 1.213938 \\ \mathrm{Ag} & -0.317841 & -0.465641 & 5.738855 \\ \mathrm{Ag} & 2.105698 & 0.145912 & 4.431126 \\ \mathrm{Ag} & -1.837392 & -2.315999 & 4.234216 \\ \mathrm{Ag} & 1.013078 & -2.521768 & 4.318080 \\ \mathrm{Ag} & 0.153228 & 4.481134 & 2.946620 \\ \mathrm{Ag} & 0.249447 & 4.579802 & 0.112052 \\ \mathrm{Ag} & 0.104640 & 2.141898 & -1.408352\end{array}$




$\begin{array}{lrrr}\mathrm{Ag} & 0.338744 & 4.587910 & -2.723968 \\ \mathrm{Ag} & 0.007893 & 2.087505 & 1.526800 \\ \mathrm{Ag} & 2.365779 & 2.639178 & 3.056186 \\ \mathrm{Ag} & 4.529997 & 0.750236 & 3.038668 \\ \mathrm{Ag} & 2.600815 & 5.041975 & 1.608497 \\ \mathrm{Ag} & 2.692988 & 5.095023 & -1.208184 \\ \mathrm{Ag} & 4.748618 & 3.211217 & 1.634052 \\ \mathrm{Ag} & -0.035840 & -0.288397 & -2.904022 \\ \mathrm{Ag} & -2.251190 & 0.656585 & -4.380280 \\ \mathrm{Ag} & -3.892339 & -1.249446 & -3.042076 \\ \mathrm{Ag} & 0.200056 & 2.175529 & -4.271194 \\ \mathrm{Ag} & 2.298053 & 0.262040 & -1.376109 \\ \mathrm{Ag} & 0.058935 & -0.250874 & -5.723820 \\ \mathrm{Ag} & 2.554481 & 2.749889 & -2.759268 \\ \mathrm{Ag} & 4.864577 & 3.268341 & -1.174940 \\ \mathrm{Ag} & 4.732751 & 0.860293 & -2.668876 \\ \mathrm{Ag} & 2.388925 & 0.307729 & -4.236398 \\ \mathrm{Ag} & -1.741104 & -2.246792 & 1.365220 \\ \mathrm{Ag} & -3.253747 & -4.123579 & -0.166093 \\ \mathrm{Ag} & -3.344444 & -4.116644 & 2.647236 \\ \mathrm{Ag} & 1.199160 & -2.401935 & -1.432933 \\ \mathrm{Ag} & 2.526723 & -4.426844 & -2.794147 \\ \mathrm{Ag} & -0.320551 & -4.264913 & -2.920987 \\ \mathrm{Ag} & 1.295321 & -2.361675 & -4.298238 \\ \mathrm{Ag} & -1.646344 & -2.192554 & -1.523445 \\ \mathrm{Ag} & -0.415196 & -4.352187 & -0.077105 \\ \mathrm{Ag} & -3.160333 & -4.010857 & -2.976948 \\ \mathrm{Ag} & 3.657530 & -1.808882 & -2.799913 \\ \mathrm{Ag} & 4.849465 & -3.873993 & -1.298504 \\ \mathrm{Ag} & 2.192506 & 0.204967 & 1.573282 \\ \mathrm{Ag} & 4.758167 & -3.925274 & 1.504403 \\ \mathrm{Ag} & 5.959646 & -1.255647 & -1.223209 \\ \mathrm{Ag} & 4.595867 & 0.794457 & 0.211744 \\ \mathrm{Ag} & 3.519055 & -1.857497 & 0.096579 \\ \mathrm{Ag} & 2.430279 & -4.540561 & 0.013098 \\ \mathrm{Ag} & 1.104419 & -2.456521 & 1.453087 \\ \mathrm{Ag} & 2.343383 & -4.530549 & 2.823221 \\ \mathrm{Ag} & -0.506594 & -4.371133 & 2.768560 \\ \mathrm{Ag} & 3.468567 & -1.914139 & 2.998490 \\ \mathrm{Ag} & 6.928637 & 1.371322 & -1.035481 \\ \mathrm{Ag} & 5.844491 & -1.314281 & 1.584415 \\ \mathrm{Ag} & -1.553806 & -2.153594 & -4.392022 \\ \mathrm{Ag} & -0.130168 & -0.320972 & 0.007301 \\ \mathrm{Ag} & -5.458863 & -3.119519 & -1.622767\end{array}$

Ag73 


\begin{tabular}{|c|c|c|c|}
\hline \multicolumn{4}{|c|}{$=-10644.4649912$ Hartree } \\
\hline $\mathrm{Ag}$ & & & \\
\hline & -1.886083 & & \\
\hline & -0.246944 & & \\
\hline & -0.244917 & -0.36 & \\
\hline & & -2.50 & \\
\hline $\mathrm{g}$ & & -2.48 & \\
\hline & -1.882609 & -2.16 & \\
\hline & & -4.58 & \\
\hline & -0.691647 & -4.3 & \\
\hline & & -4.5 & \\
\hline & 2.1 & -4.6 & \\
\hline & -0.68 & -4.3 & \\
\hline & & -2.4 & \\
\hline & 2.76 & 4.9 & \\
\hline & 2.76 & & \\
\hline & 4.56 & 0.5 & \\
\hline & & & \\
\hline & 0.3 & & \\
\hline & & & \\
\hline & -2.193 & & \\
\hline & 0.048 & & \\
\hline & & & \\
\hline & 2.1 & & \\
\hline & -2.4 & & \\
\hline & 5.7 & -1.5 & \\
\hline & 4.5 & & \\
\hline & 3.3 & -2.0 & \\
\hline & & -4.1 & \\
\hline & & & \\
\hline & 5.76 & -1.5 & \\
\hline & & & \\
\hline & 6.88 & & \\
\hline & & & \\
\hline & 4.863594 & & \\
\hline & 4.519048 & 0.58 & \\
\hline & & 0.5 & \\
\hline & & -2.0 & \\
\hline & 4.863542 & 3.02 & \\
\hline & & & \\
\hline & & 4.50 & \\
\hline & -4.361681 & & \\
\hline & -4.726479 & 1.689054 & \\
\hline & 0.048492 & & \\
\hline & 0.346707 & 4.552194 & -0.000029 \\
\hline & -2.169739 & 3.081949 & 0.000020 \\
\hline
\end{tabular}




$\begin{array}{lrrr}\mathrm{Ag} & -1.911234 & 5.486693 & 1.409465 \\ \mathrm{Ag} & -1.911212 & 5.486652 & -1.409552 \\ \mathrm{Ag} & -4.361724 & 4.106842 & 1.408770 \\ \mathrm{Ag} & -4.137174 & -1.153908 & 2.906837 \\ \mathrm{Ag} & -6.310467 & -0.175129 & 1.407971 \\ \mathrm{Ag} & -2.475651 & 0.671135 & 1.466035 \\ \mathrm{Ag} & -4.137176 & -1.153922 & -2.906831 \\ \mathrm{Ag} & -2.467560 & 0.659396 & -4.334748 \\ \mathrm{Ag} & 0.048444 & 2.075380 & -4.332500 \\ \mathrm{Ag} & -2.193143 & 3.120150 & -2.915603 \\ \mathrm{Ag} & -1.882635 & -2.164029 & -1.445351 \\ \mathrm{Ag} & 2.153446 & 0.125743 & -1.478791 \\ \mathrm{Ag} & -3.517539 & -3.942800 & -2.813949 \\ \mathrm{Ag} & -1.886070 & -2.179540 & -4.316565 \\ \mathrm{Ag} & -0.689459 & -4.312956 & -2.846431 \\ \mathrm{Ag} & -0.244898 & -0.368327 & -5.736387 \\ \mathrm{Ag} & -0.246993 & -0.352862 & -2.914624 \\ \mathrm{Ag} & 2.475861 & 2.577654 & -2.908326 \\ \mathrm{Ag} & 2.153947 & 0.118362 & -4.339779 \\ \mathrm{Ag} & 0.954846 & -2.502236 & -4.310343 \\ \mathrm{Ag} & -6.310462 & -0.175104 & -1.407880 \\ \mathrm{Ag} & -4.094626 & -1.149562 & 0.000004 \\ \mathrm{Ag} & -2.475639 & 0.671046 & -1.465979 \\ \mathrm{Ag} & -4.689262 & 1.673813 & -2.836383 \\ \mathrm{Ag} & -3.517424 & -3.942810 & 2.814033 \\ \mathrm{Ag} & -5.730305 & -2.932293 & 1.402333 \\ \mathrm{Ag} & -3.520840 & -4.002517 & -0.000013 \\ \mathrm{Ag} & -5.730375 & -2.932257 & -1.402238\end{array}$

Ag74

$\mathrm{E}=-10790.2530457$ Hartree

$\begin{array}{lrrr}\mathrm{Ag} & -0.052288 & -2.436882 & -4.384220 \\ \mathrm{Ag} & -2.465038 & -5.565313 & -1.489026 \\ \mathrm{Ag} & -1.401001 & 2.017882 & -1.539606 \\ \mathrm{Ag} & -2.413850 & -3.176818 & -2.989066 \\ \mathrm{Ag} & -2.333828 & -0.703426 & -4.384200 \\ \mathrm{Ag} & -1.390201 & 2.002266 & -4.384193 \\ \mathrm{Ag} & 2.275467 & -3.277294 & -2.989080 \\ \mathrm{Ag} & 2.224279 & -5.665789 & -1.489040 \\ \mathrm{Ag} & -0.104746 & -4.887499 & -2.917569 \\ \mathrm{Ag} & -0.052683 & -2.455968 & -1.539681 \\ \mathrm{Ag} & -0.105768 & -4.933114 & -0.080009 \\ \mathrm{Ag} & -2.389300 & -3.144652 & -0.078109 \\ \mathrm{Ag} & -4.724408 & -1.423821 & -0.079949 \\ \mathrm{Ag} & -4.680761 & -1.410708 & -2.917473 \\ \mathrm{Ag} & -3.767282 & 1.313948 & -2.989055\end{array}$




$\begin{array}{lrrr}\mathrm{Ag} & -2.352080 & -0.708867 & -1.539677 \\ \mathrm{Ag} & -4.701185 & -3.866265 & -1.488938 \\ \mathrm{Ag} & -2.788111 & 4.015646 & -2.917467 \\ \mathrm{Ag} & -2.465979 & -5.566987 & 1.328439 \\ \mathrm{Ag} & -3.729032 & 1.300563 & -0.078081 \\ \mathrm{Ag} & -5.131557 & 3.277103 & 1.328452 \\ \mathrm{Ag} & -6.054642 & 0.624615 & -1.489007 \\ \mathrm{Ag} & -5.129812 & 3.276285 & -1.488956 \\ \mathrm{Ag} & -2.346425 & -0.707183 & 4.251547 \\ \mathrm{Ag} & -3.770949 & 1.315232 & 2.830820 \\ \mathrm{Ag} & -6.056542 & 0.625099 & 1.328473 \\ \mathrm{Ag} & -2.354451 & -0.709647 & 1.387075 \\ \mathrm{Ag} & -4.686801 & -1.412501 & 2.755645 \\ \mathrm{Ag} & -2.416090 & -3.179908 & 2.830852 \\ \mathrm{Ag} & -4.702463 & -3.867663 & 1.328527 \\ \mathrm{Ag} & -0.052703 & -2.458585 & 1.387082 \\ \mathrm{Ag} & -0.104852 & -4.893888 & 2.755618 \\ \mathrm{Ag} & -0.052492 & -2.450166 & 4.251520 \\ \mathrm{Ag} & -2.814093 & 4.053183 & -0.079958 \\ \mathrm{Ag} & -1.397662 & 2.013053 & 4.251518 \\ \mathrm{Ag} & -2.791713 & 4.020911 & 2.755619 \\ \mathrm{Ag} & -1.402490 & 2.019949 & 1.387071 \\ \mathrm{Ag} & 1.530787 & 5.891161 & -1.489022 \\ \mathrm{Ag} & -1.276953 & 5.951386 & -1.489003 \\ \mathrm{Ag} & 2.957624 & 3.892538 & -2.917497 \\ \mathrm{Ag} & 0.085504 & 3.988923 & -2.989008 \\ \mathrm{Ag} & 1.486204 & 1.955955 & -1.539634 \\ \mathrm{Ag} & 2.985208 & 3.928853 & -0.079971 \\ \mathrm{Ag} & 6.077717 & 0.364960 & 1.328440 \\ \mathrm{Ag} & 3.823844 & 1.152386 & 2.830810 \\ \mathrm{Ag} & 2.314036 & -0.807132 & 4.251551 \\ \mathrm{Ag} & 0.000068 & -0.000012 & 5.658096 \\ \mathrm{Ag} & -0.000030 & -0.000053 & 2.835065 \\ \mathrm{Ag} & 2.277717 & -3.280589 & 2.830766 \\ \mathrm{Ag} & 2.321921 & -0.809843 & 1.387131 \\ \mathrm{Ag} & 4.621963 & -1.612029 & 2.755606 \\ \mathrm{Ag} & 0.000041 & 0.000039 & -0.076918 \\ \mathrm{Ag} & 1.482612 & 1.951322 & 4.251561 \\ \mathrm{Ag} & 1.487772 & 1.957986 & 1.387036 \\ \mathrm{Ag} & -2.267199 & 3.054202 & 1.328471 \\ \mathrm{Ag} & -1.277089 & 5.953262 & 1.328416 \\ \mathrm{Ag} & 0.084638 & 3.948438 & -0.078108 \\ \mathrm{Ag} & -3.244099 & -0.078144\end{array}$




$\begin{array}{lrrr}\mathrm{Ag} & 3.781282 & 1.139600 & -0.078132 \\ \mathrm{Ag} & 4.532424 & -4.065587 & 1.328360 \\ \mathrm{Ag} & 2.225272 & -5.667493 & 1.328402 \\ \mathrm{Ag} & 4.531178 & -4.064227 & -1.488995 \\ \mathrm{Ag} & 4.615967 & -1.609968 & -2.917495 \\ \mathrm{Ag} & 5.265470 & 3.053490 & -1.488949 \\ \mathrm{Ag} & 2.319482 & -0.808957 & -1.539718 \\ \mathrm{Ag} & 6.075839 & 0.364645 & -1.488991 \\ \mathrm{Ag} & 4.659028 & -1.624960 & -0.079996 \\ \mathrm{Ag} & 2.301513 & -0.802708 & -4.384270 \\ \mathrm{Ag} & 3.820161 & 1.151366 & -2.989014 \\ \mathrm{Ag} & -0.000025 & 0.000017 & -2.993319 \\ \mathrm{Ag} & 1.474654 & 1.940839 & -4.384276\end{array}$

Ag75

$E=-10936.0705809$ Hartree

$\begin{array}{lrrr}\mathrm{Ag} & -3.004869 & -5.295411 & -1.408388 \\ \mathrm{Ag} & 1.653603 & -5.859656 & -1.408409 \\ \mathrm{Ag} & -0.593423 & -4.898754 & -0.000077 \\ \mathrm{Ag} & -4.803182 & -0.941702 & -2.835034 \\ \mathrm{Ag} & -2.688680 & -2.892686 & -0.000046 \\ \mathrm{Ag} & -0.000043 & 0.000048 & -5.736580 \\ \mathrm{Ag} & 0.000046 & 0.000015 & -2.912761 \\ \mathrm{Ag} & -0.294661 & -2.432496 & -4.329863 \\ \mathrm{Ag} & -0.588601 & -4.859049 & -2.835154 \\ \mathrm{Ag} & 1.941499 & -3.489095 & -2.909142 \\ \mathrm{Ag} & -1.191426 & 2.141152 & -4.329822 \\ \mathrm{Ag} & -0.295691 & -2.441055 & -1.464526 \\ \mathrm{Ag} & -2.404621 & -0.471421 & -4.329805 \\ \mathrm{Ag} & -2.718505 & -2.924660 & -2.909119 \\ \mathrm{Ag} & 2.222464 & -1.031849 & -4.329820 \\ \mathrm{Ag} & -2.379964 & 4.277059 & -2.835075 \\ \mathrm{Ag} & -1.195647 & 2.148696 & -1.464456 \\ \mathrm{Ag} & 1.668226 & 1.794848 & -4.329775 \\ \mathrm{Ag} & -0.681599 & 6.050284 & -1.408405 \\ \mathrm{Ag} & -0.588571 & -4.859121 & 2.835093 \\ \mathrm{Ag} & -3.004817 & -5.295391 & 1.408342 \\ \mathrm{Ag} & -2.718418 & -2.924685 & 2.909144 \\ \mathrm{Ag} & -5.061917 & -3.383407 & 1.408330 \\ \mathrm{Ag} & -4.842379 & -0.949397 & 0.000018 \\ \mathrm{Ag} & -0.295662 & -2.441086 & 1.464435 \\ \mathrm{Ag} & -5.061906 & -3.383390 & -1.408332 \\ \mathrm{Ag} & -2.412932 & -0.473147 & 1.464465 \\ \mathrm{Ag} & -2.412961 & -0.473116 & -1.464495 \\ \mathrm{Ag} & -4.803107 & -0.941721 & 2.835141 \\ \mathrm{Ag} & -3.581935 & 1.663142 & 0.000023\end{array}$




$\begin{array}{lrrr}\mathrm{Ag} & -5.964750 & 1.221398 & 1.408417 \\ \mathrm{Ag} & -2.404504 & -0.471493 & 4.329782 \\ \mathrm{Ag} & -5.964803 & 1.221378 & -1.408313 \\ \mathrm{Ag} & -4.782040 & 3.768669 & -1.408370 \\ \mathrm{Ag} & -3.621640 & 1.681618 & -2.909118 \\ \mathrm{Ag} & -4.782033 & 3.768636 & 1.408432 \\ \mathrm{Ag} & -2.399359 & 4.311957 & 0.000036 \\ \mathrm{Ag} & -3.621553 & 1.681593 & 2.909145 \\ \mathrm{Ag} & -1.191417 & 2.141103 & 4.329853 \\ \mathrm{Ag} & -1.195618 & 2.148665 & 1.464505 \\ \mathrm{Ag} & -0.681592 & 6.050252 & 1.408396 \\ \mathrm{Ag} & 0.474922 & 3.920556 & -0.000013 \\ \mathrm{Ag} & -2.379888 & 4.277039 & 2.835100 \\ \mathrm{Ag} & 0.480206 & 3.963978 & -2.909109 \\ \mathrm{Ag} & -0.000045 & -0.000016 & 2.912768 \\ \mathrm{Ag} & 0.000044 & -0.000049 & 5.736587 \\ \mathrm{Ag} & -0.294653 & -2.432545 & 4.329812 \\ \mathrm{Ag} & 1.668171 & 1.794762 & 4.329832 \\ \mathrm{Ag} & 0.480167 & 3.963992 & 2.909103 \\ \mathrm{Ag} & 2.106501 & 5.712552 & 1.408328 \\ \mathrm{Ag} & 2.106512 & 5.712569 & -1.408334 \\ \mathrm{Ag} & 0.000000 & 0.000000 & 0.000004 \\ \mathrm{Ag} & 2.222409 & -1.031935 & 4.329788 \\ \mathrm{Ag} & 3.332306 & 3.585046 & 2.835170 \\ \mathrm{Ag} & 5.543510 & 2.517853 & 1.408353 \\ \mathrm{Ag} & 3.359518 & 3.614386 & 0.000022 \\ \mathrm{Ag} & 1.674018 & 1.801031 & -1.464483 \\ \mathrm{Ag} & 3.918367 & 0.768296 & 2.909152 \\ \mathrm{Ag} & 3.918342 & 0.768245 & -2.909128 \\ \mathrm{Ag} & 6.083886 & -0.238108 & 1.408328 \\ \mathrm{Ag} & 1.941477 & -3.489210 & 2.909125 \\ \mathrm{Ag} & 4.439360 & -2.061399 & 2.835096 \\ \mathrm{Ag} & 2.230230 & -1.035503 & 1.464468 \\ \mathrm{Ag} & 3.875474 & 0.759835 & -0.000031 \\ \mathrm{Ag} & 5.543522 & 2.517870 & -1.408309 \\ \mathrm{Ag} & 3.332276 & 3.585118 & -2.835076 \\ \mathrm{Ag} & 1.653546 & -5.859726 & 1.408325 \\ \mathrm{Ag} & 4.107696 & -4.494127 & 1.408303 \\ \mathrm{Ag} & 6.083897 & -0.238090 & -1.408334 \\ \mathrm{Ag} & 1.930263 & -1.035549 & -1.464509 \\ \mathrm{Ag} & -3.450963 & 0.000002 \\ \mathrm{Ag} & -1.801000 & 1.464478\end{array}$




\begin{tabular}{|c|c|c|c|}
\hline \multicolumn{4}{|c|}{ Ag76 } \\
\hline \multicolumn{4}{|c|}{$E=-11081.9009846$ Hartree } \\
\hline $\mathrm{Ag}$ & -1.436251 & & 0.064563 \\
\hline $\mathrm{Ag}$ & -3.837627 & -4.608503 & \\
\hline $\mathrm{Ag}$ & & & \\
\hline $\mathrm{Ag}$ & 1.232386 & & \\
\hline $\mathrm{Ag}$ & & & \\
\hline & & & \\
\hline $\mathrm{Ag}$ & & & \\
\hline & -0.8 & & \\
\hline $\mathrm{g}$ & & & \\
\hline $\mathrm{g}$ & & & \\
\hline $\mathrm{Ag}$ & & & \\
\hline $\mathrm{g}$ & & & \\
\hline $\mathrm{g}$ & & & \\
\hline $\mathrm{g}$ & & & \\
\hline$g$ & & & \\
\hline$g$ & & & \\
\hline & & & \\
\hline & & & \\
\hline & & & \\
\hline$g$ & & & \\
\hline$g$ & & & \\
\hline$g$ & & & \\
\hline$g$ & & & \\
\hline & -0.6 & & \\
\hline $\mathrm{g}$ & & & \\
\hline & & & \\
\hline$g$ & & & \\
\hline & & & \\
\hline & & & \\
\hline & & & \\
\hline $\mathrm{Ag}$ & & & \\
\hline & & & \\
\hline & & & \\
\hline $\mathrm{Ag}$ & & -1. & \\
\hline & & & -0.1 \\
\hline $\mathrm{Ag}$ & & & \\
\hline $\mathrm{Ag}$ & & & \\
\hline $\lg$ & & & \\
\hline $\mathrm{Ag}$ & & & -1.5 \\
\hline $\mathrm{Ag}$ & & & -2.951077 \\
\hline $\mathrm{Ag}$ & & -2.343127 & 3.022753 \\
\hline & & & \\
\hline $\mathrm{Ag}$ & & -2.323346 & 1.597528 \\
\hline $\mathrm{Ag}$ & -2.488665 & -0.000004 & -4.236626 \\
\hline
\end{tabular}




$\begin{array}{lrrr}\mathrm{Ag} & -4.901089 & 0.000026 & -2.675872 \\ \mathrm{Ag} & -3.231143 & -2.345640 & -2.783331 \\ \mathrm{Ag} & 0.009317 & -0.000047 & -2.899866 \\ \mathrm{Ag} & -0.087606 & -0.000011 & -5.721210 \\ \mathrm{Ag} & -5.606364 & -2.326009 & -1.211006 \\ \mathrm{Ag} & -0.803467 & 2.329623 & -4.286990 \\ \mathrm{Ag} & -2.393314 & -0.000026 & -1.377392 \\ \mathrm{Ag} & -3.231137 & 2.345686 & -2.783329 \\ \mathrm{Ag} & -4.760002 & 0.000048 & 0.201741 \\ \mathrm{Ag} & -7.163054 & 0.000080 & -1.046249 \\ \mathrm{Ag} & 1.424215 & 3.798236 & 2.882413 \\ \mathrm{Ag} & -3.041324 & 2.343153 & 3.022807 \\ \mathrm{Ag} & -4.695395 & 0.000063 & 3.028671 \\ \mathrm{Ag} & -2.203028 & 0.000027 & 4.428603 \\ \mathrm{Ag} & -0.611793 & 2.339825 & 1.501824 \\ \mathrm{Ag} & -0.518332 & 2.328835 & 4.363965 \\ \mathrm{Ag} & -2.287135 & -0.000018 & 1.570717 \\ \mathrm{Ag} & -5.606275 & 2.326102 & -1.210997 \\ \mathrm{Ag} & -5.490927 & 2.323457 & 1.597554 \\ \mathrm{Ag} & 1.314939 & 3.756831 & -0.026581 \\ \mathrm{Ag} & -0.708638 & 2.339277 & -1.424738 \\ \mathrm{Ag} & -1.515660 & 4.652755 & -2.767132 \\ \mathrm{Ag} & 1.232420 & 3.798617 & -2.935769 \\ \mathrm{Ag} & 3.211403 & 5.201951 & -1.498393 \\ \mathrm{Ag} & -1.436142 & 4.694043 & 0.064592 \\ \mathrm{Ag} & 0.539312 & 6.066481 & -1.409630 \\ \mathrm{Ag} & 3.303866 & 5.201904 & 1.317394 \\ \mathrm{Ag} & 0.631614 & 6.065891 & 1.406017 \\ \mathrm{Ag} & -3.095367 & 2.325841 & 0.116951 \\ \mathrm{Ag} & -3.837565 & 4.608642 & 1.552716 \\ \mathrm{Ag} & -1.330009 & 4.651728 & 2.895351 \\ \mathrm{Ag} & -3.928530 & 4.610690 & -1.262406\end{array}$

Ag77

$\mathrm{E}=-11227.7305188$ Hartree

$\begin{array}{lrrr}\mathrm{Ag} & 6.113813 & -1.404651 & -1.408437 \\ \mathrm{Ag} & 2.184119 & -1.448214 & -1.465309 \\ \mathrm{Ag} & 4.151798 & -2.880360 & -2.835583 \\ \mathrm{Ag} & 4.184188 & 0.000010 & -2.910310 \\ \mathrm{Ag} & 4.183861 & 2.903983 & 0.000037 \\ \mathrm{Ag} & 6.113852 & 1.404689 & -1.408400 \\ \mathrm{Ag} & 4.139901 & -0.000034 & -0.000028 \\ \mathrm{Ag} & 6.113881 & 1.404675 & 1.408459 \\ \mathrm{Ag} & 4.184159 & -0.000052 & 2.910319 \\ \mathrm{Ag} & 2.178295 & -1.443415 & 4.332827 \\ \mathrm{Ag} & 2.184131 & -1.448291 & 1.465182\end{array}$




\begin{tabular}{|c|c|c|c|}
\hline g & -0.567312 & -2.339663 & 1.463451 \\
\hline$g$ & -3.793930 & -4.609081 & 1.408411 \\
\hline & 6.113842 & -1.404665 & 1.408422 \\
\hline & 4.151807 & -2.880335 & 2.835613 \\
\hline & -0.566617 & -2.329263 & 4.327109 \\
\hline & 4.183854 & -2.904021 & -0.000027 \\
\hline $\mathrm{Ag}$ & -1.331129 & -4.652088 & 2.832350 \\
\hline & 0.201560 & 0.000022 & -0.000005 \\
\hline $\mathrm{Ag}$ & 1.422550 & -3.798549 & 2.910373 \\
\hline$g$ & -3.006958 & -2.327306 & 0.000002 \\
\hline Ag & -3.794054 & -4.608967 & -1.408478 \\
\hline$g$ & 0.678075 & -6.065866 & 1.408305 \\
\hline $\mathrm{Ag}$ & -1.331246 & -4.651905 & -2.832454 \\
\hline $\mathrm{Ag}$ & -1.345403 & -4.693875 & -0.000072 \\
\hline $\mathrm{Ag}$ & 3.352331 & -5.202463 & 1.408517 \\
\hline $\mathrm{Ag}$ & 3.352270 & -5.202446 & -1.408 \\
\hline $\mathrm{Ag}$ & 1.408839 & -3.756988 & 0.000010 \\
\hline $\mathrm{Ag}$ & 0.678046 & -6.065 & -1.408554 \\
\hline Ag & -3.044586 & -2.345129 & -2.903225 \\
\hline $\mathrm{Ag}$ & -0.566666 & -2.3 & -4.327140 \\
\hline $\mathrm{Ag}$ & -0.567251 & -2.339539 & -1.463435 \\
\hline $\mathrm{Ag}$ & 2.184178 & 1.448235 & -1.465198 \\
\hline $\mathrm{Ag}$ & 1.422546 & -3.798483 & -2.910478 \\
\hline Ag & 2.178315 & 1.443522 & -4.332807 \\
\hline $\mathrm{Ag}$ & 0.204025 & 0.000073 & -2.915988 \\
\hline $\mathrm{Ag}$ & 2.178338 & -1.443427 & -4.332874 \\
\hline $\mathrm{Ag}$ & 0.200245 & 0.000102 & -5.739351 \\
\hline $\mathrm{Ag}$ & 4.151775 & 2.880340 & -2.835610 \\
\hline $\mathrm{Ag}$ & -2.244138 & 0.000014 & -1.477407 \\
\hline $\mathrm{Ag}$ & -3.044562 & 2.345240 & -2.903213 \\
\hline $\mathrm{Ag}$ & -1.331234 & 4.651941 & 2.832390 \\
\hline $\mathrm{Ag}$ & -0.567283 & 2.339605 & 1.463427 \\
\hline $\mathrm{Ag}$ & -4.706767 & -0.000147 & 2.871401 \\
\hline $\mathrm{Ag}$ & -2.244149 & -0.000018 & 1.477405 \\
\hline $\mathrm{Ag}$ & -1.345411 & 4.693962 & 0.000026 \\
\hline $\mathrm{Ag}$ & -3.044664 & 2.345085 & 2.903279 \\
\hline $\mathrm{Ag}$ & -0.566730 & 2.329101 & 4.327191 \\
\hline $\mathrm{Ag}$ & -2.247724 & -0.000048 & 4.337729 \\
\hline $\mathrm{Ag}$ & -3.794080 & 4.608937 & 1.408488 \\
\hline $\mathrm{Ag}$ & -3.793983 & 4.609051 & -1.408433 \\
\hline $\mathrm{Ag}$ & -7.072903 & -0.000146 & 1.371192 \\
\hline $\mathrm{Ag}$ & -4.659393 & 0.000012 & -0.000051 \\
\hline $\mathrm{Ag}$ & -3.006871 & 2.327259 & -0.000004 \\
\hline $\mathrm{Ag}$ & -5.476370 & 2.321966 & 1.407661 \\
\hline $\mathrm{Ag}$ & -5.476339 & -2.322197 & 1.407630 \\
\hline $\mathrm{Ag}$ & -5.476331 & -2.322005 & -1.407741 \\
\hline
\end{tabular}




$\begin{array}{lrrr}\mathrm{Ag} & -7.072872 & 0.000077 & -1.371184 \\ \mathrm{Ag} & -4.706826 & 0.000077 & -2.871425 \\ \mathrm{Ag} & -5.476267 & 2.322157 & -1.407678 \\ \mathrm{Ag} & 0.678044 & 6.065903 & 1.408525 \\ \mathrm{Ag} & 1.422434 & 3.798473 & 2.910457 \\ \mathrm{Ag} & -3.044561 & -2.345184 & 2.903204 \\ \mathrm{Ag} & 0.204029 & -0.000055 & 2.916026 \\ \mathrm{Ag} & 0.200155 & -0.000042 & 5.739285 \\ \mathrm{Ag} & 2.178271 & 1.443434 & 4.332892 \\ \mathrm{Ag} & -2.247740 & 0.000083 & -4.337678 \\ \mathrm{Ag} & -1.331162 & 4.652022 & -2.832353 \\ \mathrm{Ag} & -0.566653 & 2.329323 & -4.327121 \\ \mathrm{Ag} & -0.567254 & 2.339627 & -1.463365 \\ \mathrm{Ag} & 0.678110 & 6.065915 & -1.408303 \\ \mathrm{Ag} & 1.408851 & 3.757023 & 0.000016 \\ \mathrm{Ag} & 3.352311 & 5.202416 & -1.408528 \\ \mathrm{Ag} & 3.352246 & 5.202399 & 1.408616 \\ \mathrm{Ag} & 1.422525 & 3.798537 & -2.910363 \\ \mathrm{Ag} & 2.184096 & 1.448260 & 1.465264 \\ \mathrm{Ag} & 4.151754 & 2.880363 & 2.835681\end{array}$

Ag78

$\mathrm{E}=-11373.5472633$ Hartree

$\begin{array}{lrrr}\mathrm{Ag} & -2.443867 & -0.659796 & 4.331674 \\ \mathrm{Ag} & -2.453454 & -0.672426 & 1.460649 \\ \mathrm{Ag} & -5.373264 & 3.216088 & 1.408011 \\ \mathrm{Ag} & -6.198244 & 0.531718 & 1.407902 \\ \mathrm{Ag} & -3.937851 & 1.308412 & 2.908099 \\ \mathrm{Ag} & -4.754198 & -1.444302 & 2.842437 \\ \mathrm{Ag} & -3.897857 & 1.291764 & 0.000018 \\ \mathrm{Ag} & -1.598964 & 2.100538 & 4.332615 \\ \mathrm{Ag} & -1.604050 & 2.104075 & 1.464812 \\ \mathrm{Ag} & -1.604118 & 2.104149 & -1.464752 \\ \mathrm{Ag} & -1.622074 & 6.039055 & 1.408191 \\ \mathrm{Ag} & -3.088662 & 4.082676 & 0.000082 \\ \mathrm{Ag} & -3.065028 & 4.052319 & 2.835239 \\ \mathrm{Ag} & -6.198349 & 0.531729 & -1.407905 \\ \mathrm{Ag} & -5.373238 & 3.216074 & -1.407977 \\ \mathrm{Ag} & -0.188080 & 4.130810 & -2.909336 \\ \mathrm{Ag} & -1.622093 & 6.039057 & -1.408218 \\ \mathrm{Ag} & 1.187433 & 6.081342 & -1.408234 \\ \mathrm{Ag} & -3.065070 & 4.052373 & -2.835163 \\ \mathrm{Ag} & -0.187359 & 4.086915 & 0.000053 \\ \mathrm{Ag} & -0.133198 & 0.140318 & -0.000002 \\ \mathrm{Ag} & -0.187970 & 4.130774 & 2.909414 \\ \mathrm{Ag} & 2.286154 & -3.048091 & 2.901038 \\ & & & \end{array}$




\begin{tabular}{|c|c|c|c|}
\hline g & -0.136119 & 0.140099 & 2.914076 \\
\hline$g$ & 3.657692 & 1.433898 & 2.902176 \\
\hline & 1.287090 & 2.152828 & 1.463116 \\
\hline & 5.977018 & 0.701165 & -1.407383 \\
\hline & 3.616308 & 1.427485 & -0.000041 \\
\hline g & 5.976983 & 0.701121 & 1.407275 \\
\hline & 6.823835 & -1.986098 & 1.370944 \\
\hline g & 2.203969 & -0.575304 & 1.476796 \\
\hline g & 4.561305 & -1.293526 & 860 \\
\hline & 5.037010 & 3.379691 & 1.408206 \\
\hline $\mathrm{g}$ & 2.209773 & -0.574386 & 311 \\
\hline & -0.130684 & 41183 & 8779 \\
\hline $\lg$ & -0.079122 & $-2.30 s$ & 5781 \\
\hline g & 5.037007 & 3.379675 & 3300 \\
\hline$g$ & 2.720193 & 6602 & -0.0 \\
\hline g & 1.187550 & 6.081352 & 1.408189 \\
\hline $\mathrm{lg}$ & 2.694560 & & 072 \\
\hline g & 2.694450 & 4.140995 & -2.8 \\
\hline$g$ & 1.287056 & 2.152862 & -1.46 \\
\hline $\mathrm{Ag}$ & 1.283418 & 2.14 & 877 \\
\hline$g$ & -2.412473 & -3.134989 & -2.912311 \\
\hline g & -0.089750 & -2.325339 & -1.4 \\
\hline$g$ & 2.203939 & -0.575271 & -1.476843 \\
\hline $\mathrm{g}$ & 3.657605 & 1.433898 & -2.9 \\
\hline$g$ & 2.209716 & -0.574364 & -4.337364 \\
\hline $\mathrm{Ag}$ & & -5.435579 & 34 \\
\hline$g$ & 4.561249 & 3452 & -2.8 \\
\hline$g$ & 6.823819 & -1.986141 & -1.371029 \\
\hline Ag & 2.252627 & 23419 & -0.0 \\
\hline $\lg$ & -0.015765 & -4.752828 & -2.8 \\
\hline $\mathrm{Ag}$ & 2.343055 & 5575 & 7956 \\
\hline $\mathrm{g}$ & 4.618137 & -3.739474 & -1.407257 \\
\hline $\mathrm{Ag}$ & 2.286142 & -3.048126 & -2.9 \\
\hline $\mathrm{lg}$ & -0.079181 & -2.309539 & -4.325726 \\
\hline Ag & 4.514806 & -1.280495 & -0.0( \\
\hline $\mathrm{Ag}$ & 4.618208 & -3.739427 & 7299 \\
\hline $\mathrm{Ag}$ & -2.353119 & -5.551864 & -1.435711 \\
\hline $\mathrm{Ag}$ & -2.353124 & -5.551847 & 1.435765 \\
\hline $\mathrm{Ag}$ & -2.410104 & -3.132229 & 0.000017 \\
\hline $\mathrm{Ag}$ & -4.591811 & -6.257475 & -0.000029 \\
\hline $\mathrm{Ag}$ & -2.412485 & -3.134910 & 2.912266 \\
\hline $\mathrm{Ag}$ & -0.089722 & -2.325342 & 1.458778 \\
\hline $\mathrm{Ag}$ & -0.015805 & -4.752884 & 2.839113 \\
\hline $\mathrm{Ag}$ & -0.025769 & -4.803959 & -0.000016 \\
\hline $\mathrm{Ag}$ & -4.794282 & -1.470627 & -0.000024 \\
\hline $\mathrm{Ag}$ & -4.754231 & -1.444246 & -2.842479 \\
\hline
\end{tabular}




$\begin{array}{lrrr}\mathrm{Ag} & -4.701393 & -3.912864 & 1.435895 \\ \mathrm{Ag} & -3.937818 & 1.308462 & -2.908057 \\ \mathrm{Ag} & -2.453461 & -0.672356 & -1.460669 \\ \mathrm{Ag} & -4.701447 & -3.912848 & -1.435905 \\ \mathrm{Ag} & -2.443906 & -0.659767 & -4.331572 \\ \mathrm{Ag} & -0.136125 & 0.140042 & -2.914008 \\ \mathrm{Ag} & -0.130825 & 0.141200 & -5.738828 \\ \mathrm{Ag} & 1.283381 & 2.143289 & -4.325843 \\ \mathrm{Ag} & -1.599024 & 2.100487 & -4.332576\end{array}$

Ag79

$E=-11519.3665740$ Hartree

$\begin{array}{lrrr}\mathrm{Ag} & 3.206503 & -5.413249 & -1.324300 \\ \mathrm{Ag} & -1.912162 & 0.199312 & -4.366973 \\ \mathrm{Ag} & -1.388849 & -4.509524 & -2.833922 \\ \mathrm{Ag} & 1.420426 & -3.869782 & -2.862189 \\ \mathrm{Ag} & 0.472317 & -6.065846 & -1.364960 \\ \mathrm{Ag} & -0.419614 & -2.263022 & -4.324952 \\ \mathrm{Ag} & -0.483388 & -2.258220 & -1.454693 \\ \mathrm{Ag} & 6.259417 & -1.836113 & -1.287982 \\ \mathrm{Ag} & 0.503152 & -0.000349 & -2.905441 \\ \mathrm{Ag} & 0.552219 & -0.010490 & -5.730030 \\ \mathrm{Ag} & -0.053917 & 2.378447 & -4.348258 \\ \mathrm{Ag} & 4.470301 & -0.298309 & -2.827573 \\ \mathrm{Ag} & 2.607613 & 1.285580 & -4.286828 \\ \mathrm{Ag} & 4.214667 & -3.168165 & -2.743441 \\ \mathrm{Ag} & 1.354924 & -3.814904 & 0.045085 \\ \mathrm{Ag} & 2.339470 & -1.581792 & -1.413725 \\ \mathrm{Ag} & 2.387519 & -1.593076 & -4.282841 \\ \mathrm{Ag} & 4.193628 & -3.181275 & 0.090432 \\ \mathrm{Ag} & -1.467866 & -4.535147 & 0.002670 \\ \mathrm{Ag} & -2.909618 & -2.070523 & -2.950504 \\ \mathrm{Ag} & -5.369223 & -1.825123 & -1.544383 \\ \mathrm{Ag} & -6.295811 & -3.992412 & -0.078699 \\ \mathrm{Ag} & -3.933498 & -4.270388 & 1.390538 \\ \mathrm{Ag} & -3.885998 & -4.288422 & -1.467168 \\ \mathrm{Ag} & -2.959492 & -2.055637 & -0.035541 \\ \mathrm{Ag} & -1.973301 & 0.204950 & -1.503673 \\ \mathrm{Ag} & -4.424477 & 0.419141 & -0.074102 \\ \mathrm{Ag} & -4.386763 & 0.434009 & -2.954775 \\ \mathrm{Ag} & -6.811277 & 0.644311 & -1.508358 \\ \mathrm{Ag} & -6.856251 & 0.662649 & 1.277522 \\ \mathrm{Ag} & -7.739391 & -1.582746 & -0.116161 \\ \mathrm{Ag} & -2.538961 & 2.615342 & -2.950105 \\ \mathrm{Ag} & -4.988709 & 2.812941 & -1.504194 \\ \mathrm{Ag} & 4.658597 & 2.580319 & -2.759689\end{array}$




\begin{tabular}{|c|c|c|c|}
\hline & & & \\
\hline & -0.091698 & & \\
\hline & 2.568496 & & \\
\hline & 2.008560 & & \\
\hline & 6.211398 & -1.833039 & \\
\hline & 2.287225 & -1.573992 & \\
\hline & 3.15 & & \\
\hline & 0.421168 & -6.0 & \\
\hline & & & \\
\hline & 6.46 & $0.9^{\prime}$ & -1 . \\
\hline & & & \\
\hline & 1.3 & 6.0 & \\
\hline & -3.11 & & \\
\hline & -0.6 & & \\
\hline & -1.2 & & \\
\hline & & & \\
\hline & 3.9 & 4.9 & -1 . \\
\hline & & & \\
\hline & -3.0 & $-2 .($ & \\
\hline & 1.9 & & \\
\hline & 0.35 & & \\
\hline & & & \\
\hline & -2.0 & & \\
\hline & 0.4 & 0.0 & \\
\hline & -1.4 & & \\
\hline & -5.4 & -1.8 & \\
\hline & -2.0 & & \\
\hline & 2.5 & & \\
\hline & 4.5 & & \\
\hline & & & \\
\hline & & & \\
\hline & -0.5 & -2.24 & \\
\hline & -0.5 & -2.21 & \\
\hline & 2.40 & 1.33 & \\
\hline & 4.11 & -3.14 & \\
\hline & 4.63 & 2.626 & 0.0 \\
\hline & -4.4 & 0.47 & \\
\hline & -0.17 & & \\
\hline & -3.1 & 4.99 & \\
\hline & -2.566003 & & \\
\hline & -0.140939 & 2.412978 & \\
\hline & -5.032126 & & \\
\hline & -2.628748 & 2.650715 & 2.840667 \\
\hline & & -0.283339 & \\
\hline & -0.734935 & 4.815839 & 2.808745 \\
\hline
\end{tabular}




\begin{tabular}{|c|c|c|c|}
\hline \multicolumn{4}{|c|}{ Ag80 } \\
\hline \multicolumn{4}{|c|}{$E=-11665.2200831$ Hartree } \\
\hline $\mathrm{Ag}$ & -1.244678 & -0.370330 & -4.712174 \\
\hline $\mathrm{Ag}$ & & & \\
\hline $\mathrm{Ag}$ & & & \\
\hline $\mathrm{Ag}$ & & & \\
\hline $\mathrm{Ag}$ & & & 0057 \\
\hline $\mathrm{Ag}$ & & & \\
\hline $\mathrm{Ag}$ & & & \\
\hline $\mathrm{Ag}$ & & & \\
\hline $\mathrm{Ag}$ & & & \\
\hline $\mathrm{Ag}$ & & & \\
\hline $\mathrm{Ag}$ & & & \\
\hline $\mathrm{Ag}$ & & & \\
\hline $\mathrm{Ag}$ & & & \\
\hline $\mathrm{Ag}$ & & & \\
\hline $\mathrm{Ag}$ & & & \\
\hline $\mathrm{Ag}$ & & & \\
\hline $\mathrm{Ag}$ & & & \\
\hline $\mathrm{Ag}$ & & & \\
\hline $\mathrm{Ag}$ & & & \\
\hline $\mathrm{Ag}$ & & & \\
\hline $\mathrm{Ag}$ & & & \\
\hline $\mathrm{Ag}$ & & & \\
\hline $\mathrm{Ag}$ & & & \\
\hline $\mathrm{Ag}$ & & & \\
\hline $\mathrm{Ag}$ & & & \\
\hline $\mathrm{Ag}$ & & & \\
\hline $\mathrm{Ag}$ & & & \\
\hline $\mathrm{Ag}$ & & & \\
\hline $\mathrm{Ag}$ & & & \\
\hline $\mathrm{Ag}$ & & & \\
\hline $\mathrm{Ag}$ & & & \\
\hline $\mathrm{Ag}$ & & & \\
\hline $\mathrm{Ag}$ & & & \\
\hline $\mathrm{Ag}$ & & & \\
\hline $\mathrm{Ag}$ & & & \\
\hline $\mathrm{Ag}$ & & & \\
\hline $\mathrm{Ag}$ & & & \\
\hline $\mathrm{Ag}$ & & & 916 \\
\hline $\mathrm{Ag}$ & & & \\
\hline $\mathrm{Ag}$ & & & \\
\hline Ag & -0.31 & -4.197693 & \\
\hline $\mathrm{Ag}$ & -0.467745 & & \\
\hline $\mathrm{Ag}$ & -1.975195 & -4.237564 & -3.761023 \\
\hline $\mathrm{Ag}$ & -2.705080 & -5.707893 & -1.446459 \\
\hline
\end{tabular}




$\begin{array}{lrrr}\mathrm{Ag} & -0.779659 & 1.057241 & 0.000010 \\ \mathrm{Ag} & -4.649490 & -0.682172 & -2.362001 \\ \mathrm{Ag} & -1.097837 & 3.432012 & -1.449908 \\ \mathrm{Ag} & -1.097785 & 3.431931 & 1.450175 \\ \mathrm{Ag} & -2.686768 & 3.346545 & 3.790416 \\ \mathrm{Ag} & -2.687128 & 3.346654 & -3.790185 \\ \mathrm{Ag} & -1.496887 & 5.819454 & 0.000000 \\ \mathrm{Ag} & -3.670062 & 4.668630 & 1.452880 \\ \mathrm{Ag} & -3.670211 & 4.668611 & -1.452632 \\ \mathrm{Ag} & -3.813661 & 0.821874 & -4.640713 \\ \mathrm{Ag} & -3.300414 & 2.286846 & 0.000105 \\ \mathrm{Ag} & -5.809381 & 3.494065 & 0.000303 \\ \mathrm{Ag} & -4.861692 & 2.185535 & -2.346266 \\ \mathrm{Ag} & -2.296012 & 0.963383 & -2.363901 \\ \mathrm{Ag} & -3.539904 & -1.998526 & 4.621606 \\ \mathrm{Ag} & -5.351364 & -2.203236 & 0.000042 \\ \mathrm{Ag} & -1.997636 & -1.883671 & 2.339253 \\ \mathrm{Ag} & -5.625200 & 0.670011 & 0.000197 \\ \mathrm{Ag} & -4.323767 & -3.531626 & -2.337083 \\ \mathrm{Ag} & -4.649339 & -0.682243 & 2.362133 \\ \mathrm{Ag} & -2.295779 & 0.963126 & 2.364093 \\ \mathrm{Ag} & -3.813181 & 0.821561 & 4.641007 \\ \mathrm{Ag} & -1.244405 & -0.371085 & 4.712148 \\ \mathrm{Ag} & -4.861448 & 2.185415 & 2.346712 \\ \mathrm{Ag} & -3.540031 & -1.998155 & -4.621579 \\ \mathrm{Ag} & -1.997716 & -1.883380 & -2.339280 \\ \mathrm{Ag} & -3.079328 & -0.554481 & 0.000038 \\ \mathrm{Ag} & -5.005646 & -5.006511 & -0.000071 \\ \mathrm{Ag} & -4.323711 & -3.531830 & 2.337027 \\ \mathrm{Ag} & -2.733490 & -3.364878 & -0.000111 \\ \mathrm{Ag} & -2.705046 & -5.708001 & 1.446168 \\ \mathrm{Ag} & -1.975078 & -4.237895 & 3.760745 \\ \mathrm{Ag} & 0.400963 & -2.614164 & 3.856545 \\ \mathrm{Ag} & 0.262073 & -0.214173 & 2.369505 \\ \mathrm{Ag} & -0.073826 & 2.158964 & 3.878888 \\ \mathrm{Ag} & -1.455092 & 5.734725 & 2.849489\end{array}$

Ag81

$E=-11811.0446374$ Hartree

$\begin{array}{lrrr}\mathrm{Ag} & -4.346251 & -3.404131 & -2.334475 \\ \mathrm{Ag} & -5.358896 & -2.067939 & -0.000024 \\ \mathrm{Ag} & -4.657319 & -0.541695 & 2.360688 \\ \mathrm{Ag} & -2.636718 & 3.465605 & 3.789474 \\ \mathrm{Ag} & -5.608976 & 0.820749 & -0.000080 \\ \mathrm{Ag} & -2.025254 & -1.762895 & 2.349807 \\ \mathrm{Ag} & -2.279084 & 1.079376 & 2.362988\end{array}$




$\begin{array}{lrrr}\mathrm{Ag} & -3.803466 & 0.959151 & 4.638215 \\ \mathrm{Ag} & -3.573985 & -1.860704 & 4.627695 \\ \mathrm{Ag} & -2.032958 & -4.113765 & -3.797195 \\ \mathrm{Ag} & -0.380668 & -4.049529 & -1.517425 \\ \mathrm{Ag} & -2.033005 & -4.113645 & 3.797324 \\ \mathrm{Ag} & -0.492758 & -1.667381 & -0.000004 \\ \mathrm{Ag} & -0.380715 & -4.049459 & 1.517588 \\ \mathrm{Ag} & -2.728315 & -5.629576 & 1.457496 \\ \mathrm{Ag} & -2.728263 & -5.629703 & -1.457437 \\ \mathrm{Ag} & -5.029770 & -4.880892 & 0.000004 \\ \mathrm{Ag} & -4.346280 & -3.404131 & 2.334512 \\ \mathrm{Ag} & -2.752575 & -3.267786 & 0.000021 \\ \mathrm{Ag} & -3.081381 & -0.436335 & -0.000047 \\ \mathrm{Ag} & -2.025239 & -1.762979 & -2.349763 \\ \mathrm{Ag} & -5.756046 & 3.651318 & -0.000078 \\ \mathrm{Ag} & -4.657249 & -0.541771 & -2.360832 \\ \mathrm{Ag} & -3.573910 & -1.860842 & -4.627791 \\ \mathrm{Ag} & -4.828658 & 2.331805 & 2.344539 \\ \mathrm{Ag} & -3.264122 & 2.411679 & -0.000094 \\ \mathrm{Ag} & -1.043167 & 3.529993 & -1.450251 \\ \mathrm{Ag} & -0.759071 & 1.155351 & -0.000069 \\ \mathrm{Ag} & -3.599167 & 4.798808 & -1.451958 \\ \mathrm{Ag} & -2.636481 & 3.465571 & -3.789643 \\ \mathrm{Ag} & -0.037275 & 2.245464 & -3.878011 \\ \mathrm{Ag} & -2.279095 & 1.079305 & -2.363044 \\ \mathrm{Ag} & -1.368569 & 5.835384 & -2.850013 \\ \mathrm{Ag} & -3.599181 & 4.798839 & 1.451843 \\ \mathrm{Ag} & -1.043193 & 3.530027 & 1.450155 \\ \mathrm{Ag} & 1.865878 & -2.341923 & -1.453841 \\ \mathrm{Ag} & -1.368724 & 5.835400 & 2.849999 \\ \mathrm{Ag} & -1.409128 & 5.921455 & -0.000048 \\ \mathrm{Ag} & -4.828583 & 2.331714 & -2.344748 \\ \mathrm{Ag} & 0.377776 & -2.519808 & -3.882296 \\ \mathrm{Ag} & -1.249197 & -0.262481 & -4.716304 \\ \mathrm{Ag} & -3.803218 & 0.959018 & -4.638323 \\ \mathrm{Ag} & 0.264182 & -0.134412 & -2.372204 \\ \mathrm{Ag} & 3.662791 & 4.023572 & 1.462900 \\ \mathrm{Ag} & 1.184756 & 4.657183 & 2.881441 \\ \mathrm{Ag} & 1.677300 & -0.017947 & -0.000018 \\ \mathrm{Ag} & 4.106002 & -1.249746 & 0.000032 \\ \mathrm{Ag} & -6.531831 & -2.474358 & 0.000123 \\ \mathrm{Ag} & 1.865824 & -2.341892 & 1.453986 \\ \mathrm{Ag} & -6.347576 & 0.000104\end{array}$




$\begin{array}{lrrr}\mathrm{Ag} & 0.264102 & -0.134347 & 2.372284 \\ \mathrm{Ag} & 2.054451 & -4.653927 & 2.887767 \\ \mathrm{Ag} & -1.249320 & -0.262225 & 4.716331 \\ \mathrm{Ag} & 3.102990 & 0.115678 & 2.334601 \\ \mathrm{Ag} & 0.377609 & -2.519565 & 3.882386 \\ \mathrm{Ag} & 4.523995 & 0.246748 & 4.648829 \\ \mathrm{Ag} & 5.339967 & 1.792564 & 2.367967 \\ \mathrm{Ag} & 3.314935 & -2.240285 & 3.838519 \\ \mathrm{Ag} & 5.599558 & -1.139519 & 2.368235 \\ \mathrm{Ag} & 5.599605 & -1.139687 & -2.368007 \\ \mathrm{Ag} & 3.852857 & 1.632829 & -0.000037 \\ \mathrm{Ag} & 6.366649 & 0.404258 & 0.000005 \\ \mathrm{Ag} & 1.672091 & -0.005335 & 4.761070 \\ \mathrm{Ag} & -0.037338 & 2.245709 & 3.877892 \\ \mathrm{Ag} & 1.464291 & 2.312846 & 1.447510 \\ \mathrm{Ag} & 6.022844 & 3.268959 & -0.000032 \\ \mathrm{Ag} & 2.896124 & 2.481282 & 3.833097 \\ \mathrm{Ag} & 3.315008 & -2.240468 & -3.838293 \\ \mathrm{Ag} & 2.054507 & -4.654105 & -2.887505 \\ \mathrm{Ag} & 5.340089 & 1.792493 & -2.367983 \\ \mathrm{Ag} & 4.524095 & 0.246429 & -4.648786 \\ \mathrm{Ag} & 3.103066 & 0.115578 & -2.334604 \\ \mathrm{Ag} & 1.205213 & 4.747085 & -0.000134 \\ \mathrm{Ag} & 1.464299 & 2.312810 & -1.447535 \\ \mathrm{Ag} & 1.672245 & -0.005550 & -4.760965 \\ \mathrm{Ag} & 1.184817 & 4.656999 & -2.881679 \\ \mathrm{Ag} & 3.662810 & 4.023451 & -1.463044 \\ \mathrm{Ag} & 2.896205 & 2.480995 & -3.833213\end{array}$

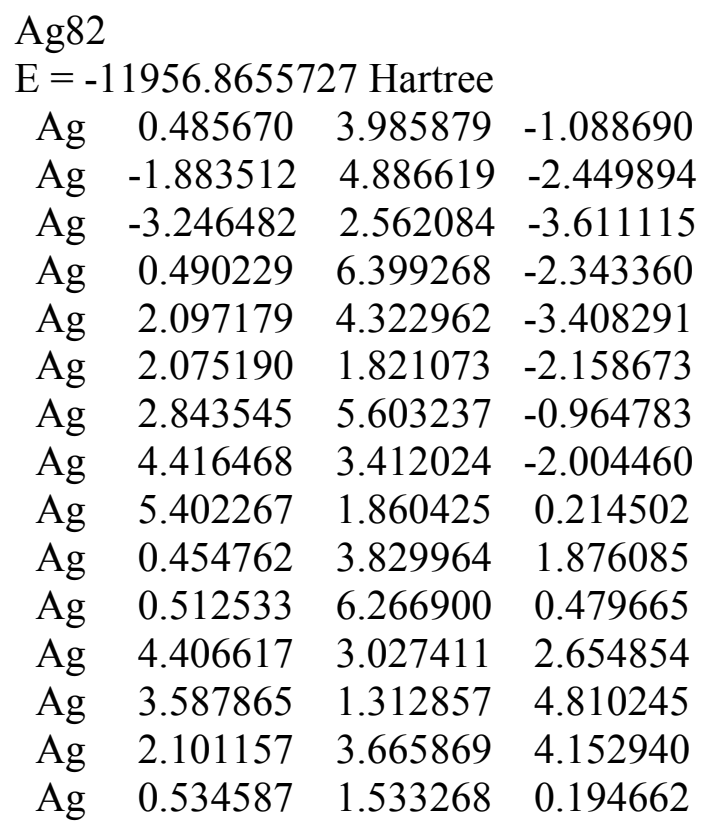




\begin{tabular}{|c|c|c|c|}
\hline $\lg$ & 2.820753 & 5.353600 & 1.936055 \\
\hline$g$ & 5.126304 & 4.672435 & 0.447836 \\
\hline & 2.051122 & 1.432663 & 2.533991 \\
\hline & 4.653937 & 0.163116 & 2.440381 \\
\hline g & 2.831248 & 3.101030 & 0.318572 \\
\hline & -1.927857 & 4.721497 & 0.425129 \\
\hline $\mathrm{Ag}$ & -1.970261 & 4.357185 & 3.295320 \\
\hline & -4.267446 & 3.512764 & 1.780408 \\
\hline $\mathrm{Ag}$ & -1.819952 & 2.414734 & -1.224263 \\
\hline g & -5.589984 & 0.983052 & 2.469629 \\
\hline $\mathrm{Ag}$ & -6.477689 & 2.538245 & 0.218954 \\
\hline $\mathrm{Ag}$ & -4.083058 & 1.259323 & 0.124710 \\
\hline $\mathrm{Ag}$ & -6.379941 & -0.334753 & -0.024060 \\
\hline Ag & -5.563806 & 1.389784 & 9759 \\
\hline $\mathrm{Ag}$ & -4.250122 & 3.766251 & -1.133811 \\
\hline $\mathrm{Ag}$ & -1.821130 & 2.172684 & 1.66 \\
\hline $\mathrm{Ag}$ & -3.284743 & 1.901692 & 4.032787 \\
\hline $\mathrm{Ag}$ & -4.5567 & -0.6155 & 4.6 \\
\hline $\mathrm{Ag}$ & -0.279974 & -0.148152 & 2.410320 \\
\hline Ag & -1.700530 & -0.43 & $4.7^{\prime}$ \\
\hline Ag & 1.228641 & -0.245612 & 624 \\
\hline $\mathrm{Ag}$ & -0.340460 & & \\
\hline $\mathrm{Ag}$ & -0.036642 & -2.6499 & 3.7 \\
\hline $\mathrm{Ag}$ & -1.684345 & -0.028689 & 0.028283 \\
\hline $\mathrm{Ag}$ & 3.757597 & -1.5034 & 4.5 \\
\hline $\mathrm{Ag}$ & -3.122195 & -0.322562 & 2.338151 \\
\hline $\mathrm{Ag}$ & -5.396697 & -1.943795 & 2.221598 \\
\hline $\mathrm{Ag}$ & -2.975330 & -2.810204 & 3.631558 \\
\hline $\mathrm{Ag}$ & 3.791590 & -0.727081 & -4.660569 \\
\hline $\mathrm{Ag}$ & 5.586968 & -1.025509 & -0.0 \\
\hline $\mathrm{Ag}$ & 3.090968 & 0.275567 & 5118 \\
\hline $\mathrm{Ag}$ & 4.674476 & 0.554499 & -2.267164 \\
\hline $\mathrm{Ag}$ & 2.257464 & -1.011463 & -2.4 \\
\hline $\mathrm{Ag}$ & 3.621980 & 2.089617 & -4.417225 \\
\hline $\mathrm{Ag}$ & 0.725158 & -1.261560 & -0.065267 \\
\hline $\mathrm{Ag}$ & 2.568330 & -3.275254 & -4.029940 \\
\hline $\mathrm{Ag}$ & 3.491689 & -4.823014 & -1.810801 \\
\hline $\mathrm{Ag}$ & 4.778436 & -2.314849 & -2.489768 \\
\hline $\mathrm{Ag}$ & 3.203877 & -2.561546 & -0.164043 \\
\hline $\mathrm{Ag}$ & 5.667621 & -3.848875 & -0.262428 \\
\hline $\mathrm{Ag}$ & -0.255193 & 0.263966 & -2.318698 \\
\hline $\mathrm{Ag}$ & -3.895975 & -1.619312 & -0.119923 \\
\hline $\mathrm{Ag}$ & -0.001373 & -1.987907 & -4.021666 \\
\hline $\mathrm{Ag}$ & -3.752449 & -3.879969 & -1.780627 \\
\hline $\mathrm{Ag}$ & 1.244071 & -5.689254 & -3.295487 \\
\hline $\mathrm{Ag}$ & -1.515968 & -2.229111 & -1.613522 \\
\hline
\end{tabular}




$\begin{array}{lrrr}\mathrm{Ag} & -6.102181 & -3.197207 & -0.266010 \\ \mathrm{Ag} & 1.271647 & -6.013369 & -0.460623 \\ \mathrm{Ag} & -2.939665 & -2.158096 & -4.008752 \\ \mathrm{Ag} & -1.281621 & -4.448256 & -3.240782 \\ \mathrm{Ag} & -5.373768 & -1.539624 & -2.497939 \\ \mathrm{Ag} & -1.654532 & 0.371664 & -4.714916 \\ \mathrm{Ag} & 0.963556 & -3.503781 & -1.710339 \\ \mathrm{Ag} & -3.099795 & 0.074611 & -2.314858 \\ \mathrm{Ag} & -0.309952 & 2.775308 & -3.623523 \\ \mathrm{Ag} & 1.267051 & 0.552261 & -4.642995 \\ \mathrm{Ag} & -4.510133 & 0.179367 & -4.635480 \\ \mathrm{Ag} & 2.539838 & -3.907634 & 3.523082 \\ \mathrm{Ag} & 0.951808 & -3.746907 & 1.183988 \\ \mathrm{Ag} & 2.238028 & -1.405080 & 2.299495 \\ \mathrm{Ag} & 3.480729 & -5.065557 & 1.085263 \\ \mathrm{Ag} & 4.760347 & -2.704795 & 2.181589 \\ \mathrm{Ag} & -3.766921 & -4.129122 & 1.135513 \\ \mathrm{Ag} & -1.309250 & -4.939255 & 2.503277 \\ \mathrm{Ag} & -1.530050 & -2.477054 & 1.273298 \\ \mathrm{Ag} & -1.317676 & -4.782628 & -0.376424 \\ \mathrm{Ag} & 1.222116 & -6.164062 & 2.389117\end{array}$

Ag83

$E=-12102.6818934$ Hartree

$\begin{array}{llll}\mathrm{Ag} & 0.630171 & -1.396048 & 0.000025\end{array}$

Ag $\quad 1.242924 \quad-0.000254 \quad-4.719335$

$\begin{array}{llll}\mathrm{Ag} & -1.603479 & -4.694702 & -2.882784\end{array}$

$\begin{array}{llll}\mathrm{Ag} & -1.682172 & -2.336360 & -1.449893\end{array}$

Ag $\quad-3.123220 \quad-0.000143 \quad-2.335550$

$\begin{array}{llll}\mathrm{Ag} & -1.687422 & -0.000271 & -4.762003\end{array}$

Ag $\quad-3.121868 \quad-2.373560 \quad-3.834401$

$\begin{array}{llll}\mathrm{Ag} & -0.177716 & -2.396441 & -3.878505\end{array}$

$\begin{array}{llll}\mathrm{Ag} & -5.496906 & 1.473486 & -2.368612\end{array}$

$\begin{array}{llll}\mathrm{Ag} & -4.547961 & -0.000329 & -4.648712\end{array}$

$\begin{array}{llll}\text { Ag } & 0.878429 & -6.176406 & 0.000013\end{array}$

$\begin{array}{llll}\mathrm{Ag} & 2.313179 & -3.828129 & -3.790117\end{array}$

$\begin{array}{llll}\mathrm{Ag} & 3.156244 & -5.244833 & 1.454619\end{array}$

$\begin{array}{llll}\mathrm{Ag} & 0.845461 & -6.086868 & -2.855097\end{array}$

$\begin{array}{lllll}\mathrm{Ag} & 0.714273 & -3.768612 & -1.454553\end{array}$

$\begin{array}{llll}\text { Ag } & 3.156399 & -5.244763 & -1.454561\end{array}$

$\begin{array}{llll}\text { Ag } & -4.020241 & 3.845671 & -1.463676\end{array}$

$\begin{array}{lllll}\mathrm{Ag} & 3.687045 & -1.417987 & -4.633991\end{array}$

$\begin{array}{lllll}\mathrm{Ag} & 4.590787 & -2.882269 & -2.342947\end{array}$

$\begin{array}{llll}\mathrm{Ag} & 5.402456 & -4.283961 & 0.000127\end{array}$

$\begin{array}{llll}\mathrm{Ag} & 3.024699 & -2.844196 & 0.000017\end{array}$

$\begin{array}{llll}\text { Ag } & 2.155577 & -1.424828 & -2.360474\end{array}$ 


\begin{tabular}{|c|c|c|c|}
\hline$g$ & 0.878425 & 6.176504 & -0.000010 \\
\hline $\mathrm{Ag}$ & 3.686850 & 1.417904 & -4.634028 \\
\hline & 0.714292 & 3.768544 & -1.454756 \\
\hline $\mathrm{Ag}$ & 0.845229 & 6.086960 & -2.855073 \\
\hline & -0.177722 & 2.395948 & -3.878708 \\
\hline & -1.603434 & 4.694323 & -2.883260 \\
\hline$g$ & -1.682098 & 2.336242 & -1.450127 \\
\hline & -0.274391 & -0.000175 & -2.375113 \\
\hline g & 2.312949 & 3.828051 & -3.790193 \\
\hline & 5.504110 & -1.451295 & 0.000023 \\
\hline $\mathrm{Ag}$ & 3.098174 & -0.000021 & -0.000011 \\
\hline g & 5.402424 & 4.283989 & -0.000072 \\
\hline $\mathrm{Ag}$ & 3.156231 & 5.244804 & -1.454663 \\
\hline $\mathrm{Ag}$ & 3.024705 & 2.844139 & -0.000026 \\
\hline Ag & 4.590641 & 2.882178 & -2.343041 \\
\hline $\mathrm{Ag}$ & 5.504055 & 1.451377 & -0.000023 \\
\hline $\mathrm{Ag}$ & 4.668766 & 0.000073 & -2.364066 \\
\hline $\mathrm{Ag}$ & 2.155528 & 1.424778 & -2.360566 \\
\hline $\mathrm{Ag}$ & 0.630080 & 1.396023 & -0.000005 \\
\hline $\mathrm{Ag}$ & -3.121808 & 2.373088 & -3.834622 \\
\hline $\mathrm{Ag}$ & -3.123243 & 0.000127 & 2.335543 \\
\hline Ag & -5.496915 & -1.473770 & -2.368464 \\
\hline $\mathrm{Ag}$ & -4.020301 & -3.845876 & -1.463189 \\
\hline $\mathrm{Ag}$ & -6.397876 & 0.000035 & 0.000052 \\
\hline $\mathrm{Ag}$ & -3.121839 & -2.373065 & 3.834 \\
\hline $\mathrm{Ag}$ & -5.496907 & 1.473809 & 2.368455 \\
\hline $\mathrm{Ag}$ & -6.306639 & 2.88 & -0.000251 \\
\hline $\mathrm{Ag}$ & -4.003624 & 1.448519 & -0.000091 \\
\hline $\mathrm{Ag}$ & -1.695095 & -0.000020 & -0.000003 \\
\hline $\mathrm{Ag}$ & -4.003728 & -1.448435 & 0.000154 \\
\hline $\mathrm{Ag}$ & -4.548036 & 0.000271 & 4.648676 \\
\hline Ag & -5.496916 & -1.473447 & 2.368604 \\
\hline $\mathrm{Ag}$ & -6.306706 & -2.886193 & 0.000137 \\
\hline $\mathrm{Ag}$ & 0.845214 & -6.086849 & 2.855065 \\
\hline $\mathrm{Ag}$ & -1.630482 & -4.781282 & 0.000254 \\
\hline $\mathrm{Ag}$ & -1.682168 & -2.336188 & 1.450154 \\
\hline $\mathrm{Ag}$ & -4.020298 & -3.845729 & 1.463681 \\
\hline $\mathrm{Ag}$ & -1.603488 & -4.694331 & 2.883313 \\
\hline $\mathrm{Ag}$ & -0.177681 & -2.396045 & 3.878709 \\
\hline $\mathrm{Ag}$ & 2.312993 & -3.828069 & 3.790169 \\
\hline $\mathrm{Ag}$ & 0.714312 & -3.768546 & 1.454692 \\
\hline $\mathrm{Ag}$ & -3.121779 & 2.373584 & 3.834410 \\
\hline $\mathrm{Ag}$ & -4.020229 & 3.845959 & 1.463182 \\
\hline $\mathrm{Ag}$ & -1.687398 & 0.000277 & 4.762095 \\
\hline $\mathrm{Ag}$ & -1.682071 & 2.336336 & 1.449862 \\
\hline $\mathrm{Ag}$ & -0.274404 & 0.000115 & 2.375132 \\
\hline
\end{tabular}




$\begin{array}{lrrr}\mathrm{Ag} & -0.177688 & 2.396344 & 3.878506 \\ \mathrm{Ag} & 3.686965 & 1.417935 & 4.633987 \\ \mathrm{Ag} & 1.242976 & 0.000139 & 4.719322 \\ \mathrm{Ag} & 2.313093 & 3.828090 & 3.790079 \\ \mathrm{Ag} & 4.668711 & 0.000029 & 2.364080 \\ \mathrm{Ag} & 2.155523 & 1.424811 & 2.360502 \\ \mathrm{Ag} & 4.590785 & 2.882265 & 2.342862 \\ \mathrm{Ag} & 4.590692 & -2.882162 & 2.343008 \\ \mathrm{Ag} & 2.155549 & -1.424717 & 2.360653 \\ \mathrm{Ag} & 3.686900 & -1.417859 & 4.634135 \\ \mathrm{Ag} & 3.156384 & 5.244790 & 1.454562 \\ \mathrm{Ag} & 0.845428 & 6.086877 & 2.855056 \\ \mathrm{Ag} & 0.714298 & 3.768547 & 1.454560 \\ \mathrm{Ag} & -1.630457 & 4.781221 & -0.000266 \\ \mathrm{Ag} & -1.603513 & 4.694618 & 2.882739\end{array}$

Ag84

$\mathrm{E}=-12248.4999189$ Hartree

$\begin{array}{llll}\text { Ag } & -4.682201 & -1.581564 & 4.518685\end{array}$

$\begin{array}{llll}\mathrm{Ag} & -1.819887 & -1.685590 & 4.697640\end{array}$

$\begin{array}{llll}\mathrm{Ag} & -3.182797 & -0.873368 & 2.363527\end{array}$

$\begin{array}{llll}\text { Ag } & 0.652890 & 1.132942 & 0.702531\end{array}$

$\begin{array}{llll}\mathrm{Ag} & -1.680626 & -0.161268 & 0.191253\end{array}$

$\begin{array}{llll}\mathrm{Ag} & -1.673104 & 3.326110 & 4.420994\end{array}$

$\begin{array}{llll}\mathrm{Ag} & -3.197990 & 0.885166 & 4.545754\end{array}$

$\begin{array}{llll}\text { Ag } & -0.246237 & 0.826608 & 4.666799\end{array}$

$\begin{array}{llll}\text { Ag } & -4.002685 & 3.061923 & 2.745812\end{array}$

$\begin{array}{llll}\text { Ag } & -6.258937 & 2.679122 & 0.997114\end{array}$

$\begin{array}{llll}\mathrm{Ag} & -3.974819 & 1.261543 & 0.598674\end{array}$

$\begin{array}{llll}\text { Ag } & -5.377741 & 2.091491 & -1.678724\end{array}$

Ag $\quad-3.025766 \quad-1.141064 \quad-4.236247$

$\begin{array}{llll}\mathrm{Ag} & -4.370933 & 1.417652 & -4.291770\end{array}$

$\begin{array}{llll}\mathrm{Ag} & -4.012595 & -1.468633 & -0.337562\end{array}$

$\begin{array}{llll}\mathrm{Ag} & -6.341162 & -2.768365 & -0.859808\end{array}$

$\begin{array}{llll}\text { Ag } & -4.118792 & -4.202175 & 0.278666\end{array}$

$\begin{array}{llll}\text { Ag } & -3.269000 & -3.594496 & 3.025595\end{array}$

$\begin{array}{llll}\mathrm{Ag} & -5.582901 & -2.215032 & 1.859254\end{array}$

$\begin{array}{llll}\mathrm{Ag} & -5.542083 & 0.563964 & 2.809058\end{array}$

$\begin{array}{llll}\mathrm{Ag} & -1.669541 & 1.588876 & 2.317254\end{array}$

$\begin{array}{llll}\mathrm{Ag} & -6.389830 & -0.042467 & 0.064512\end{array}$

$\begin{array}{llll}\mathrm{Ag} & -4.032829 & -3.276451 & -2.493198\end{array}$

$\begin{array}{llll}\mathrm{Ag} & -5.420581 & -0.688990 & -2.625615\end{array}$

$\begin{array}{lllll}\mathrm{Ag} & -1.508849 & 1.379966 & -4.345605\end{array}$

$\begin{array}{llll}\mathrm{Ag} & -1.198976 & 4.147891 & -4.841909\end{array}$

$\begin{array}{lllll}\mathrm{Ag} & -3.022382 & 0.639493 & -2.063457\end{array}$

$\begin{array}{llll}\mathrm{Ag} & 1.617432 & 3.998042 & -4.708237\end{array}$ 


\begin{tabular}{|c|c|c|c|}
\hline g & 0.229391 & 5.925003 & -3.180399 \\
\hline $\mathrm{Ag}$ & 2.511417 & 4.620860 & -2.100289 \\
\hline & 0.000476 & 3.224252 & -2.574673 \\
\hline $\mathrm{Ag}$ & -1.617462 & 2.520690 & -0.404393 \\
\hline & -3.925413 & 4.010873 & -0.015409 \\
\hline & -2.943100 & 3.386995 & -2.740751 \\
\hline $\mathrm{Ag}$ & 1.031435 & 6.507083 & -0.542207 \\
\hline & 1.001580 & 5.652958 & 2.175754 \\
\hline $\mathrm{Ag}$ & 3.299368 & 5.172481 & 0.602409 \\
\hline & 0.824466 & 3.844125 & 0.093417 \\
\hline $\mathrm{Ag}$ & -1.457555 & 5.228252 & -1.041950 \\
\hline g & 3.202247 & 4.219238 & 3.338547 \\
\hline $\mathrm{Ag}$ & 2.305596 & 2.117124 & 5.078833 \\
\hline $\mathrm{Ag}$ & 0.724091 & 2.952981 & 2.905640 \\
\hline Ag & -1.555112 & 4.373557 & 1.732680 \\
\hline $\mathrm{Ag}$ & 0.614145 & -1.569292 & -0.182119 \\
\hline $\mathrm{Ag}$ & 3.554508 & -3.107345 & 4.247190 \\
\hline $\mathrm{Ag}$ & 2.072727 & -2.358662 & 6705 \\
\hline $\mathrm{Ag}$ & 3.590656 & -0.429084 & 5.146329 \\
\hline $\mathrm{Ag}$ & 5.348099 & -4.384238 & -1.021252 \\
\hline $\mathrm{Ag}$ & -0.317673 & -3.700535 & 3.128660 \\
\hline $\mathrm{Ag}$ & 4.501666 & -3.783482 & 1.624392 \\
\hline $\mathrm{Ag}$ & 2.980862 & -2.975568 & -0.605844 \\
\hline $\mathrm{Ag}$ & & -1.738339 & \\
\hline $\mathrm{Ag}$ & 2.196987 & -5.050525 & 2.653007 \\
\hline $\mathrm{Ag}$ & -0.335470 & -0.951988 & 2.474251 \\
\hline $\mathrm{Ag}$ & 4.581875 & 1.673428 & 3.465259 \\
\hline $\mathrm{Ag}$ & 2.108849 & 0.353225 & 2.962035 \\
\hline $\mathrm{Ag}$ & 4.602554 & -1.061655 & 2.564185 \\
\hline $\mathrm{Ag}$ & 3.093118 & -0.268949 & 0.296348 \\
\hline $\mathrm{Ag}$ & 5.480188 & -1.697825 & -0.115508 \\
\hline $\mathrm{Ag}$ & 0.610286 & -4.351543 & 0.436159 \\
\hline $\mathrm{Ag}$ & 3.047041 & -5.686526 & -0.018863 \\
\hline $\mathrm{Ag}$ & 4.635832 & -2.286100 & -2.803303 \\
\hline $\mathrm{Ag}$ & 3.142630 & -4.760376 & -2.773657 \\
\hline $\mathrm{Ag}$ & 0.859910 & -5.027331 & -4.446084 \\
\hline $\mathrm{Ag}$ & -1.696085 & -4.691848 & -1.341728 \\
\hline $\mathrm{Ag}$ & 0.709937 & -3.315539 & -2.336367 \\
\hline $\mathrm{Ag}$ & 0.817885 & -6.031949 & -1.805571 \\
\hline $\mathrm{Ag}$ & 2.391705 & -2.649181 & -4.542107 \\
\hline $\mathrm{Ag}$ & -1.588499 & -3.700422 & -4.038706 \\
\hline $\mathrm{Ag}$ & -1.673848 & -1.919692 & -1.930232 \\
\hline $\mathrm{Ag}$ & -1.814612 & -5.496729 & 1.429754 \\
\hline $\mathrm{Ag}$ & -1.741523 & -2.839970 & 0.812189 \\
\hline $\mathrm{Ag}$ & 3.062291 & 2.434404 & 1.213986 \\
\hline $\mathrm{Ag}$ & 5.473640 & 3.734732 & 1.722220 \\
\hline
\end{tabular}




$\begin{array}{lrrr}\mathrm{Ag} & 2.273151 & 1.851612 & -1.504027 \\ \mathrm{Ag} & 4.714439 & 3.174252 & -0.962217 \\ \mathrm{Ag} & 4.750885 & 0.455157 & -1.908952 \\ \mathrm{Ag} & 2.225998 & -0.857665 & -2.406203 \\ \mathrm{Ag} & 1.387535 & 1.241471 & -4.238955 \\ \mathrm{Ag} & 3.840959 & 2.562878 & -3.659787 \\ \mathrm{Ag} & 3.832351 & -0.149019 & -4.527591 \\ \mathrm{Ag} & 5.521865 & 1.050361 & 0.811417 \\ \mathrm{Ag} & -0.093942 & -1.256329 & -4.225927 \\ \mathrm{Ag} & -0.181982 & 0.508390 & -2.019557\end{array}$

Ag85

$E=-12394.2855889$ Hartree

$\begin{array}{rrrr}\mathrm{Ag} & -3.208346 & 2.437305 & -1.412013 \\ \mathrm{Ag} & -4.438323 & 2.895625 & -3.891634 \\ \mathrm{Ag} & -5.778297 & 2.133630 & -0.204608 \\ \mathrm{Ag} & -3.623133 & 0.356688 & -4.818291 \\ \mathrm{Ag} & -1.702473 & 2.234387 & -3.875696 \\ \mathrm{Ag} & -5.086337 & 4.476589 & -1.665652 \\ \mathrm{Ag} & -0.881696 & -0.348195 & -4.798035 \\ \mathrm{Ag} & -0.033079 & 3.840653 & 2.235302 \\ \mathrm{Ag} & 1.064333 & 1.538536 & -3.799434 \\ \mathrm{Ag} & -2.543818 & 4.780412 & -2.886899 \\ \mathrm{Ag} & -0.444265 & 1.750991 & -1.347445 \\ \mathrm{Ag} & 2.310704 & 1.051709 & -1.260951 \\ \mathrm{Ag} & 2.702463 & 4.665976 & 2.040638 \\ \mathrm{Ag} & 1.460034 & 3.593776 & -0.256689 \\ \mathrm{Ag} & -1.294885 & 4.275046 & -0.350650 \\ \mathrm{Ag} & 0.224048 & 4.108722 & -2.807368 \\ \mathrm{Ag} & 4.174530 & 4.445555 & -0.348868 \\ \mathrm{Ag} & 3.813829 & 0.805683 & -3.682147 \\ \mathrm{Ag} & 0.790073 & 1.284050 & 1.246092 \\ \mathrm{Ag} & 2.975125 & 3.391434 & -2.693864 \\ \mathrm{Ag} & 0.614467 & 6.131168 & 0.717048 \\ \mathrm{Ag} & -0.633382 & 6.587771 & -1.746449 \\ \mathrm{Ag} & 2.103285 & 5.901281 & -1.649758 \\ \mathrm{Ag} & -1.979777 & 5.838561 & 1.850484 \\ \mathrm{Ag} & -3.206196 & 6.309289 & -0.630977 \\ \mathrm{Ag} & 6.181043 & 2.941227 & 0.996887 \\ \mathrm{Ag} & 4.723041 & 3.164645 & 3.381916 \\ \mathrm{Ag} & 2.038645 & 2.355989 & 3.584038 \\ \mathrm{Ag} & 3.516874 & 2.106629 & 1.101906 \\ \mathrm{Ag} & 4.333285 & -0.440454 & 0.133904 \\ \mathrm{Ag} & 5.575253 & 0.614330 & 2.486848 \\ \mathrm{Ag} & 6.984506 & 0.401191 & 0.066582 \\ \mathrm{Ag} & 5.037793 & 1.910802 & -1.357555\end{array}$




$\begin{array}{lrrr}\mathrm{Ag} & 5.840308 & -0.679371 & -2.297321 \\ \mathrm{Ag} & 4.079759 & 0.838408 & 4.851902 \\ \mathrm{Ag} & -0.697620 & 1.503302 & 3.734737 \\ \mathrm{Ag} & -3.317294 & 1.168980 & 4.799596 \\ \mathrm{Ag} & -3.928629 & 4.028206 & 0.869794 \\ \mathrm{Ag} & -2.667764 & 3.532355 & 3.370234 \\ \mathrm{Ag} & -1.964004 & 1.978823 & 1.157031 \\ \mathrm{Ag} & 2.840030 & -0.216707 & 2.595001 \\ \mathrm{Ag} & 1.375508 & -0.004697 & 5.063224 \\ \mathrm{Ag} & -1.343596 & -0.835819 & 5.213656 \\ \mathrm{Ag} & 3.137869 & -1.519032 & -2.199476 \\ \mathrm{Ag} & 4.901897 & -2.136822 & 2.333257 \\ \mathrm{Ag} & 3.664574 & -3.286372 & 0.018784 \\ \mathrm{Ag} & 2.187299 & -3.066426 & 2.444773 \\ \mathrm{Ag} & 6.340193 & -2.353898 & -0.100914 \\ \mathrm{Ag} & 5.112549 & -3.475092 & -2.406638 \\ \mathrm{Ag} & 2.671900 & -3.702788 & -3.932865 \\ \mathrm{Ag} & 1.633885 & -5.227295 & 0.641734 \\ \mathrm{Ag} & 1.162541 & -3.442288 & -1.495319 \\ \mathrm{Ag} & 3.127574 & -5.411287 & -1.760965 \\ \mathrm{Ag} & 1.891067 & -1.051485 & -4.713022 \\ \mathrm{Ag} & 4.617468 & -1.760694 & -4.591560 \\ \mathrm{Ag} & -1.600977 & -2.759788 & -1.587665 \\ \mathrm{Ag} & -2.374737 & -0.113060 & -2.373221 \\ \mathrm{Ag} & 0.679887 & -5.601049 & -3.165061 \\ \mathrm{Ag} & -0.095357 & -3.010029 & -4.051043 \\ \mathrm{Ag} & 0.386822 & -0.807368 & -2.306712 \\ \mathrm{Ag} & -0.334523 & -3.217995 & 0.966059 \\ \mathrm{Ag} & 1.638786 & -1.283763 & 0.264334 \\ \mathrm{Ag} & -4.508073 & -3.758366 & 2.947530 \\ \mathrm{Ag} & -6.472739 & -0.653361 & -0.313332 \\ \mathrm{Ag} & -2.569657 & -0.373476 & 2.618301 \\ \mathrm{Ag} & 0.147906 & -1.061094 & 2.746562 \\ \mathrm{Ag} & -2.309944 & -5.155043 & 1.630870 \\ \mathrm{Ag} & 0.172639 & -4.960653 & 3.063551 \\ \mathrm{Ag} & -0.819823 & -5.408050 & -0.786146 \\ \mathrm{Ag} & -4.007460 & -1.578010 & 4.666218 \\ \mathrm{Ag} & -1.827693 & -3.013649 & 3.458046 \\ \mathrm{Ag} & 0.704574 & -2.801611 & 4.845048 \\ \mathrm{Ag} & 3.402629 & -1.912250 & 4.729989 \\ \mathrm{Ag} & -4.954357 & -5.812361 & 1.182276 \\ \mathrm{Ag} & -4.394796 & 1.652745 & 2.330810 \\ \mathrm{Ag} & -2.097487 & -2.476112 \\ \mathrm{Ag} & -4.905718 & -3.295303\end{array}$




$\begin{array}{lrrr}\mathrm{Ag} & -2.850065 & -2.293630 & -4.102487 \\ \mathrm{Ag} & -1.096144 & -0.577555 & 0.169408 \\ \mathrm{Ag} & -3.108265 & -2.538229 & 0.856988 \\ \mathrm{Ag} & -3.587986 & -4.685236 & -0.922613 \\ \mathrm{Ag} & -5.764727 & -3.291113 & 0.418112 \\ \mathrm{Ag} & -5.280588 & -1.124320 & 2.196129\end{array}$

\begin{tabular}{lrrr}
$\mathrm{Ag} 86$ & \multicolumn{3}{c}{} \\
$\mathrm{E}=-12540.1228438$ & Hartree & \\
$\mathrm{Ag}$ & 3.227296 & 4.629198 & -3.168596 \\
$\mathrm{Ag}$ & 7.103356 & -1.365417 & -0.297808 \\
$\mathrm{Ag}$ & 5.504587 & 0.544245 & -1.625629 \\
$\mathrm{Ag}$ & 4.364475 & -1.904721 & -0.565337 \\
$\mathrm{Ag}$ & 6.027018 & -3.754495 & 0.721525 \\
$\mathrm{Ag}$ & 3.289283 & -4.398909 & 0.478482 \\
$\mathrm{Ag}$ & 4.314712 & 5.220782 & -0.643257 \\
$\mathrm{Ag}$ & 4.651486 & 3.240961 & 1.340348 \\
$\mathrm{Ag}$ & 3.498354 & 2.610533 & -1.249130 \\
$\mathrm{Ag}$ & 6.231244 & 3.194906 & -0.991515 \\
$\mathrm{Ag}$ & 1.547882 & 4.736340 & -0.903474 \\
$\mathrm{Ag}$ & -1.040110 & -3.794322 & 3.746917 \\
$\mathrm{Ag}$ & -4.226958 & -0.449226 & 4.323477 \\
$\mathrm{Ag}$ & -2.675995 & -1.886271 & 2.426868 \\
$\mathrm{Ag}$ & -3.650790 & -3.196931 & 4.647156 \\
$\mathrm{Ag}$ & -1.604561 & -1.311026 & 5.025503 \\
$\mathrm{Ag}$ & -0.606752 & 0.039544 & 2.747469 \\
$\mathrm{Ag}$ & -2.145796 & 1.473818 & 4.630382 \\
$\mathrm{Ag}$ & 3.189282 & 1.187239 & 5.510533 \\
$\mathrm{Ag}$ & 0.481147 & 0.618644 & 5.327775 \\
$\mathrm{Ag}$ & -3.233463 & 0.885679 & 2.039102 \\
$\mathrm{Ag}$ & -4.743468 & 2.320763 & 3.872766 \\
$\mathrm{Ag}$ & 2.960457 & 3.244707 & 3.621440 \\
$\mathrm{Ag}$ & -2.790164 & 5.530712 & 0.733605 \\
$\mathrm{Ag}$ & -0.916571 & 2.106917 & 0.795418 \\
$\mathrm{Ag}$ & -5.043859 & 4.355485 & 1.954564 \\
$\mathrm{Ag}$ & 0.187822 & 2.706387 & 3.417245 \\
$\mathrm{Ag}$ & -0.131315 & 4.745239 & 1.424476 \\
$\mathrm{Ag}$ & -2.445834 & 3.561142 & 2.713015 \\
$\mathrm{Ag}$ & 2.667337 & 5.255278 & 1.646017 \\
$\mathrm{Ag}$ & 1.053264 & -1.872497 & 4.088337 \\
$\mathrm{Ag}$ & 5.518349 & -1.306690 & 2.031523 \\
$\mathrm{Ag}$ & 4.375074 & -3.721458 & 3.007160 \\
$\mathrm{Ag}$ & 2.716098 & -1.888948 & 1.744467 \\
$\mathrm{Ag}$ & 3.825207 & 0.579333 & 0.709247 \\
$\mathrm{Ag}$ & 6.559589 & 1.134506 & 0.937117 \\
& 1.047358 & 0.002925 & 0.394344
\end{tabular}




\begin{tabular}{|c|c|c|c|}
\hline $\mathrm{g}$ & 1.609363 & -4.321378 & 2.772520 \\
\hline & -0.053358 & -2.456904 & 1.465427 \\
\hline & 2.132944 & 0.602199 & 3.006590 \\
\hline & 3.813976 & -1.267238 & 4.305445 \\
\hline & 4.908527 & 1.175517 & 3.287167 \\
\hline & 1.844804 & 2.670946 & 1.060386 \\
\hline & -2.324516 & 3.522408 & -3.716726 \\
\hline & -1.227821 & 3927 & -1.175193 \\
\hline & -3.147663 & 8002 & -4.41 \\
\hline & -2.271196 & -3.671483 & -3.704846 \\
\hline & -0.063 & -0.6 & -2.23 \\
\hline & -1.187225 & -1.220271 & -4.786 \\
\hline & 3.290546 & -2.5 & -3.194 \\
\hline & 0.512043 & -3.1 & -3.476786 \\
\hline & -0.57 & -5.4 & -2.3 \\
\hline & 4.386388 & -0.0 & -4.161 \\
\hline & 2.706448 & -0.0 & $-1.925^{\prime}$ \\
\hline g & 0.731 & 2.0 & -1.566 \\
\hline & -2.032 & 1.4 & -1.847 \\
\hline & 5.14 & 2.6 & -3.5 \\
\hline & 0.463206 & 4.09 & -3.480177 \\
\hline & 2.41 & & -3.88 \\
\hline & -0.369737 & 1.4 & -4.17 \\
\hline & 1.60 & -0.6 & -4.5 \\
\hline g & 6.02 & -1.9 & -2.826 \\
\hline & 4.94 & -4.3 & -1.806 \\
\hline $\mathrm{g}$ & 1.61 & -2.5 & -0.871 \\
\hline & 2.19 & -4.9 & -2.096428 \\
\hline g & -3.93 & -1.7 & -5.013492 \\
\hline & -3.865 & 4.9 & -1.801714 \\
\hline$g$ & -1.15 & -3.0 & -1.13 \\
\hline g & -4.729421 & -3.80 & 2.16 \\
\hline $\mathrm{Ag}$ & -2.134323 & -4.426316 & 1.202247 \\
\hline$g$ & -5.777036 & -4.36 & -0.342066 \\
\hline$g$ & -3.769143 & -2.48 & -0.138803 \\
\hline $\lg$ & -4.898055 & -3.11 & -2.699341 \\
\hline & -6.403389 & -1.63 & -0.783610 \\
\hline $\mathrm{Ag}$ & -5.346152 & -1.060065 & 1.786514 \\
\hline & -5.454377 & -0.33 & -3.133484 \\
\hline $\mathrm{Ag}$ & -1.700953 & -0.547236 & 0.110885 \\
\hline$\Delta \sigma$ & 0.502891 & -4.951681 & 0.218825 \\
\hline $\mathrm{Ag}$ & -3.213354 & -4.983499 & -1.361060 \\
\hline $\mathrm{Ag}$ & -4.672778 & 2.342218 & -2.527189 \\
\hline $\mathrm{Ag}$ & -2.836721 & -1.182524 & -2.491206 \\
\hline $\mathrm{Ag}$ & -5.875694 & 1.717358 & 1.364579 \\
\hline$\Delta \sigma$ & -6.900983 & 1.142730 & -1.194793 \\
\hline
\end{tabular}




$\begin{array}{llll}\mathrm{Ag} & -4.340516 & 0.280151 & -0.561411 \\ \mathrm{Ag} & -6.118035 & 3.769402 & -0.568676 \\ \mathrm{Ag} & -3.541453 & 2.912958 & 0.090958\end{array}$

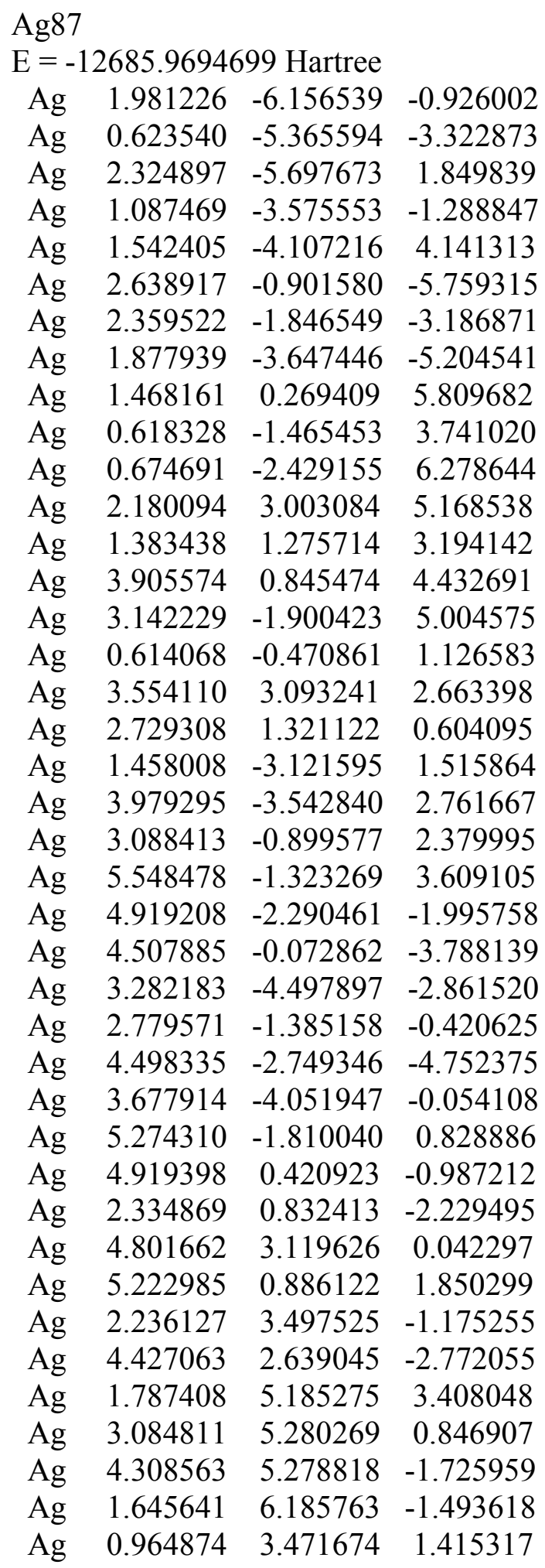




\begin{tabular}{|c|c|c|c|}
\hline g & -1.118092 & 0.714199 & 4.646902 \\
\hline $\mathrm{Ag}$ & 0.410743 & 6.157263 & 1.103543 \\
\hline & 2.590663 & 1.803244 & -4.811698 \\
\hline g & 2.481189 & 4.511632 & -3.745813 \\
\hline & 0.377006 & 0.005727 & -4.346134 \\
\hline & 0.219985 & -0.948036 & -1.676906 \\
\hline Ag & -0.209030 & -5.329577 & 0.639756 \\
\hline & -0.391334 & -2.762597 & -3.765010 \\
\hline $\mathrm{Ag}$ & -2.459104 & -1.825242 & -2.043297 \\
\hline & -1.723320 & 0.919748 & -2.615474 \\
\hline Ag & -2.428152 & -0.836386 & -4.672433 \\
\hline $\mathrm{Ag}$ & -3.022700 & -4.554066 & 0.884625 \\
\hline $\mathrm{Ag}$ & -3.144081 & -3.556087 & -4.025600 \\
\hline $\mathrm{Ag}$ & -1.648121 & -4.524260 & -1.7 \\
\hline $\lg$ & -1.857007 & -1.048038 & 2.52 \\
\hline Ag & -1.040025 & -3.749930 & 2.9 \\
\hline Ag & -1.048280 & -2.691931 & 0.309084 \\
\hline $\mathrm{Ag}$ & -3.850575 & -2.891462 & \\
\hline Ag & -4.168364 & 1.381394 & -3.871869 \\
\hline $\mathrm{Ag}$ & -1.649 & 1.9 & -5.16 \\
\hline $\mathrm{Ag}$ & -1.797899 & -0.065612 & -0.042765 \\
\hline $\mathrm{Ag}$ & -4.195306 & 0.360687 & -1.2 \\
\hline $\mathrm{Ag}$ & -4.929527 & 7198 & -3.2 \\
\hline $\mathrm{Ag}$ & -6.595520 & 0.788050 & -2.457520 \\
\hline $\mathrm{Ag}$ & -3.772923 & -1.862040 & 0.535 \\
\hline $\mathrm{Ag}$ & -6.293557 & 1.282375 & 0.356120 \\
\hline $\mathrm{Ag}$ & -5.858849 & -0.982660 & 2.180641 \\
\hline $\mathrm{Ag}$ & -5.737881 & -3.649053 & 1.108193 \\
\hline $\mathrm{Ag}$ & -1.851296 & -2.035389 & 5.106 \\
\hline $\mathrm{Ag}$ & -4.479275 & -3.669740 & -1.481924 \\
\hline $\mathrm{Ag}$ & -6.251517 & -1.457730 & -0.6 \\
\hline $\mathrm{Ag}$ & 0.355975 & 2.712512 & -3.281168 \\
\hline $\mathrm{Ag}$ & -2.540228 & 2.557944 & -0.365017 \\
\hline $\mathrm{Ag}$ & -0.187675 & 5.402956 & -3.493916 \\
\hline $\mathrm{Ag}$ & -2.429048 & 3.630549 & -2.988861 \\
\hline $\mathrm{Ag}$ & 0.177305 & 1.728960 & -0.633384 \\
\hline $\mathrm{Ag}$ & -1.818129 & 4.404862 & 1.682953 \\
\hline $\mathrm{Ag}$ & -0.518292 & 4.447867 & -0.930697 \\
\hline $\mathrm{Ag}$ & -4.601408 & 3.503433 & 1.262987 \\
\hline $\mathrm{Ag}$ & -3.280194 & 5.160940 & -0.692179 \\
\hline $\mathrm{Ag}$ & -5.830054 & 1.719530 & 3.163362 \\
\hline $\mathrm{Ag}$ & -1.125347 & 1.681007 & 1.983632 \\
\hline $\mathrm{Ag}$ & -0.358926 & 3.448811 & 4.003817 \\
\hline $\mathrm{Ag}$ & -3.148279 & 2.618367 & 3.638695 \\
\hline $\mathrm{Ag}$ & -3.818223 & 0.830069 & 1.567773 \\
\hline $\mathrm{Ag}$ & -4.989901 & 3.028192 & -1.609868 \\
\hline
\end{tabular}




$$
\begin{array}{llll}
\text { Ag } & -3.899028 & -0.152268 & 4.197313
\end{array}
$$

\begin{tabular}{|c|c|c|c|}
\hline \multicolumn{4}{|c|}{ Ag89 } \\
\hline \multicolumn{4}{|c|}{$E=-12977.6272197$ Hartree } \\
\hline $\mathrm{Ag}$ & -0.950290 & 1.885276 & \\
\hline $\mathrm{Ag}$ & & & \\
\hline $\mathrm{Ag}$ & -1.601956 & & \\
\hline $\mathrm{Ag}$ & & & \\
\hline $\mathrm{Ag}$ & -0.950207 & -0.9 & \\
\hline $\mathrm{Ag}$ & & & \\
\hline $\mathrm{Ag}$ & & & \\
\hline $\mathrm{Ag}$ & & & \\
\hline $\mathrm{Ag}$ & & & \\
\hline $\mathrm{Ag}$ & & & \\
\hline $\mathrm{Ag}$ & & & \\
\hline $\mathrm{Ag}$ & & & \\
\hline $\mathrm{Ag}$ & & & \\
\hline $\mathrm{Ag}$ & & & \\
\hline $\mathrm{Ag}$ & & & \\
\hline $\mathrm{Ag}$ & & & \\
\hline $\mathrm{Ag}$ & & & \\
\hline $\mathrm{Ag}$ & & & \\
\hline & & & \\
\hline $\mathrm{Ag}$ & & & \\
\hline $\mathrm{Ag}$ & -1. & & \\
\hline $\mathrm{Ag}$ & & & \\
\hline $\mathrm{Ag}$ & & & \\
\hline $\mathrm{Ag}$ & & & \\
\hline $\mathrm{Ag}$ & & & \\
\hline $\mathrm{Ag}$ & & & \\
\hline $\mathrm{Ag}$ & -0.6 & & \\
\hline $\mathrm{Ag}$ & & & \\
\hline $\mathrm{Ag}$ & & & \\
\hline $\mathrm{Ag}$ & -2.6 & & \\
\hline $\mathrm{Ag}$ & & & \\
\hline $\mathrm{Ag}$ & & & \\
\hline $\mathrm{Ag}$ & & & \\
\hline $\mathrm{Ag}$ & & & \\
\hline $\mathrm{Ag}$ & & & \\
\hline $\mathrm{Ag}$ & -1.60 & & \\
\hline $\mathrm{Ag}$ & & & \\
\hline $\mathrm{Ag}$ & -4.9 & -0.00 & \\
\hline $\mathrm{Ag}$ & & & \\
\hline $\mathrm{Ag}$ & & -4.747023 & -0.630723 \\
\hline & & -4.106948 & \\
\hline Ag & -3.305024 & 3.507207 & 2.697203 \\
\hline
\end{tabular}




$\begin{array}{lrrr}\mathrm{Ag} & -4.908884 & 2.866766 & 0.373359 \\ \mathrm{Ag} & -1.601637 & 4.064409 & 4.909389 \\ \mathrm{Ag} & -2.584300 & 3.053707 & -1.261068 \\ \mathrm{Ag} & -3.255514 & 5.233250 & 0.387949 \\ \mathrm{Ag} & -4.825869 & 4.579145 & -1.890935 \\ \mathrm{Ag} & -2.651730 & 1.321019 & 1.016058 \\ \mathrm{Ag} & -4.908734 & 1.096745 & 2.675064 \\ \mathrm{Ag} & -4.825480 & -0.651994 & 4.911493 \\ \mathrm{Ag} & -2.583968 & -0.434739 & 3.275308 \\ \mathrm{Ag} & -3.255187 & 1.718542 & 4.958322 \\ \mathrm{Ag} & 0.108172 & -0.587564 & 4.427107 \\ \mathrm{Ag} & -2.219570 & -0.796117 & 5.998218 \\ \mathrm{Ag} & -0.642006 & -3.123335 & 5.389943 \\ \mathrm{Ag} & -0.642090 & 1.609229 & 6.018043 \\ \mathrm{Ag} & -0.340407 & -0.217395 & 1.637579 \\ \mathrm{Ag} & -0.950022 & 1.938079 & 3.295938 \\ \mathrm{Ag} & -1.601757 & 5.788312 & 2.667617 \\ \mathrm{Ag} & 1.764013 & 4.747230 & 0.629413 \\ \mathrm{Ag} & -0.340548 & 1.526893 & -0.630598 \\ \mathrm{Ag} & -0.642481 & 6.229441 & 0.009888 \\ \mathrm{Ag} & 1.763922 & 2.919792 & -3.795811 \\ \mathrm{Ag} & 0.107731 & 4.127781 & -1.704683 \\ \mathrm{Ag} & 2.314220 & 2.500732 & -1.032802 \\ \mathrm{Ag} & 4.517442 & 2.750307 & -2.727507 \\ \mathrm{Ag} & -2.220068 & 5.592471 & -2.309595 \\ \mathrm{Ag} & 0.724065 & 4.308762 & 3.313390 \\ \mathrm{Ag} & -0.950150 & 3.683051 & 1.026806 \\ \mathrm{Ag} & 6.138194 & 0.437412 & -3.294134 \\ \mathrm{Ag} & 2.816234 & 4.976659 & -2.055264 \\ \mathrm{Ag} & 2.314355 & -0.355950 & 2.681929 \\ \mathrm{Ag} & 1.828140 & 0.000043 & -0.000086 \\ \mathrm{Ag} & 1.764255 & 1.827434 & 4.426378 \\ \mathrm{Ag} & 4.517779 & -1.929588 & 3.358090 \\ \mathrm{Ag} & 1.764407 & -2.918678 & 3.796376 \\ \mathrm{Ag} & 2.816687 & -0.708290 & 5.337326 \\ \mathrm{Ag} & 3.986035 & -1.540557 & 0.636154 \\ \mathrm{Ag} & 1.330416 & 2.151212 & 1.654193 \\ \mathrm{Ag} & 6.138327 & 2.634245 & 2.025390 \\ \mathrm{Ag} & 4.517441 & 3.873363 & -0.008104 \\ \mathrm{Ag} & 6.138511 & -3.071023 & 1.268156 \\ \mathrm{Ag} & 3.517698 & 0.987024 & 3.745283 \\ \mathrm{Ag} & 1.321461 & 1.016000 \\ \mathrm{Ag} & 0.219412 & -1.652500 \\ \mathrm{Ag} & -0.222994 & 1.681173 \\ \mathrm{Ag} & & & \\ \mathrm{Ag} & & & \\ \mathrm{Ag} & & & \end{array}$




\begin{tabular}{|c|c|c|c|}
\hline $\mathrm{Ag}$ & 6.220616 & -1.344377 & \\
\hline \multicolumn{4}{|c|}{ Ag90 } \\
\hline \multicolumn{4}{|c|}{$E=-13123.4511236$ Hartree } \\
\hline $\mathrm{Ag}$ & 2.289798 & 3.800010 & 2.391027 \\
\hline $\mathrm{Ag}$ & 3.744287 & & \\
\hline $\mathrm{Ag}$ & -0.154969 & & -0.000005 \\
\hline $\mathrm{Ag}$ & & & \\
\hline $\mathrm{Ag}$ & & & \\
\hline $\mathrm{Ag}$ & 0.16 & 5.7 & \\
\hline $\mathrm{Ag}$ & -2.14 & & \\
\hline $\mathrm{Ag}$ & $-1.3 \xi$ & & -0.0 \\
\hline $\mathrm{Ag}$ & & & \\
\hline $\lg$ & & 0.7 & -0 . \\
\hline $\lg$ & & & \\
\hline $\mathrm{Ag}$ & & & \\
\hline & & & \\
\hline $\lg$ & & & \\
\hline $\mathrm{Ag}$ & & & \\
\hline $\mathrm{Ag}$ & & & \\
\hline $\lg$ & -0.5 & & \\
\hline $\lg$ & & & \\
\hline $\lg$ & & & \\
\hline $\lg$ & & & \\
\hline $\mathrm{Ag}$ & & & \\
\hline & & & \\
\hline $\mathrm{Ag}$ & & & \\
\hline $\mathrm{Ag}$ & & & \\
\hline $\mathrm{Ag}$ & & -1 . & \\
\hline Ag & & & \\
\hline $\mathrm{Ag}$ & & -4.1 & \\
\hline $\mathrm{A} \xi$ & & & \\
\hline & & & \\
\hline $\mathrm{Ag}$ & -0.8 & & \\
\hline $\mathrm{Ag}$ & & & \\
\hline $\mathrm{Ag}$ & & & \\
\hline $\mathrm{Ag}$ & & & \\
\hline $\mathrm{Ag}$ & & -1.7 & \\
\hline $\mathrm{Ag}$ & & & \\
\hline $\mathrm{Ag}$ & 4.052328 & -3.72 & 2.367180 \\
\hline $\mathrm{Ag}$ & & -1.7 & \\
\hline $\mathrm{Ag}$ & & & \\
\hline $\mathrm{Ag}$ & 3.712769 & -2.265546 & \\
\hline $\mathrm{Ag}$ & & -0.314892 & \\
\hline $\mathrm{Ag}$ & 2.853117 & -4.951432 & 0.000111 \\
\hline $\mathrm{Ag}$ & -0.761315 & -0.786855 & 6.175808 \\
\hline
\end{tabular}




\begin{tabular}{|c|c|c|c|}
\hline g & 2.097969 & -1.730723 & -2.343833 \\
\hline$g$ & 4.052275 & -3.721769 & -2.367098 \\
\hline & 1.732980 & -0.315119 & -4.785723 \\
\hline & -2.594483 & 5.011479 & -2.350701 \\
\hline g & -4.547492 & 3.070119 & -1.443414 \\
\hline & -2.540388 & 1.098293 & -1.448941 \\
\hline $\mathrm{Ag}$ & -0.885642 & 0.577230 & -3.783121 \\
\hline & -1.347914 & 2.014459 & -6.07 \\
\hline g & -1.001962 & 4.443202 & -4.660708 \\
\hline & -4.547436 & 3.070212 & 1.44 \\
\hline $\mathrm{Ag}$ & -2.594499 & 5.011543 & 2.35 \\
\hline$g$ & -2.540262 & 1.09 & 1.4 \\
\hline $\mathrm{Ag}$ & -4.130713 & 5.480668 & 0.000014 \\
\hline $\mathrm{Ag}$ & -4.959892 & 0.5 & 0.0 \\
\hline $\lg$ & -4.884995 & 0.576415 & -2.894673 \\
\hline $\mathrm{Ag}$ & -5.215271 & $-1.8 \mathrm{~S}$ & -1.45 \\
\hline g & -2.799263 & -1.371005 & -2.863 \\
\hline $\mathrm{Ag}$ & -5.130370 & -1.88 & -4.2 \\
\hline $\mathrm{Ag}$ & -3.832755 & -3.986581 & -2.883545 \\
\hline $\mathrm{Ag}$ & -1.319117 & -4.89 & \\
\hline $\mathrm{Ag}$ & -0.463620 & $-0.86 ?$ & -1.4 \\
\hline $\mathrm{Ag}$ & -0.207127 & -2.308 & -3.8 \\
\hline $\mathrm{Ag}$ & -2.991804 & 2.567 & -3.8 \\
\hline $\mathrm{Ag}$ & -0.761561 & -0.786875 & $-6.1^{\prime}$ \\
\hline $\mathrm{Ag}$ & -2.621964 & -2.775222 & -5.2 \\
\hline g & -3.281557 & 0.063 & -5.235888 \\
\hline $\mathrm{Ag}$ & 2.418015 & -3.123892 & -4.6 \\
\hline $\mathrm{Ag}$ & 1.175794 & -4.424831 & 2.396237 \\
\hline $\mathrm{Ag}$ & 1.175706 & -4.424900 & -2.396037 \\
\hline $\mathrm{Ag}$ & 0.939633 & -2.95 & 0.0 \\
\hline $\mathrm{Ag}$ & -0.206987 & -2.308333 & 3.872580 \\
\hline $\mathrm{Ag}$ & -2.621748 & -2.775195 & 5.242794 \\
\hline $\mathrm{Ag}$ & -3.832671 & -3.9865 & 2.88 \\
\hline $\mathrm{Ag}$ & -1.319057 & -4.891708 & 3.790909 \\
\hline $\mathrm{Ag}$ & -5.130219 & -1.883048 & 4.292515 \\
\hline $\mathrm{Ag}$ & -1.435037 & -3.469395 & 1.431197 \\
\hline $\mathrm{Ag}$ & -0.028628 & -5.565017 & 0.000101 \\
\hline $\mathrm{Ag}$ & -2.440826 & -6.023688 & 1.413911 \\
\hline $\mathrm{Ag}$ & -2.799153 & -1.370991 & 2.863111 \\
\hline $\mathrm{Ag}$ & -2.991660 & 2.567711 & 3.830275 \\
\hline $\mathrm{Ag}$ & -3.281373 & 0.063945 & 5.235993 \\
\hline $\mathrm{Ag}$ & -0.463507 & -0.863875 & 1.435042 \\
\hline $\mathrm{Ag}$ & -4.884886 & 0.576468 & 2.894771 \\
\hline $\mathrm{Ag}$ & -1.435023 & -3.469470 & -1.431159 \\
\hline $\mathrm{Ag}$ & -2.866580 & -1.374390 & 0.000019 \\
\hline $\mathrm{Ag}$ & -2.440736 & -6.023692 & -1.413690 \\
\hline
\end{tabular}




$$
\begin{array}{llll}
\mathrm{Ag} & -3.887167 & -4.018565 & 0.000059 \\
\mathrm{Ag} & -5.215208 & -1.893356 & 1.452385
\end{array}
$$

Ag91

$\mathrm{E}=-13269.2721209$ Hartree

$\begin{array}{llll}\text { Ag } & 2.358352 & -1.164189 & 3.020286\end{array}$

$\begin{array}{llll}\text { Ag } & 0.000145 & -0.201912 & 4.552775\end{array}$

$\begin{array}{llll}\mathrm{Ag} & 2.393470 & -1.043102 & 5.772850\end{array}$

$\begin{array}{llll}\mathrm{Ag} & 6.099238 & -1.720911 & 1.831284\end{array}$

$\begin{array}{llll}\mathrm{Ag} & 4.684036 & -1.902252 & 4.279786\end{array}$

$\begin{array}{llll}\mathrm{Ag} & 0.000179 & -0.462116 & 1.724231\end{array}$

$\begin{array}{llll}\mathrm{Ag} & -0.000386 & 1.811419 & 0.205961\end{array}$

$\begin{array}{llll}\mathrm{Ag} & -1.473373 & 4.258685 & 3.950667\end{array}$

$\begin{array}{llll}\mathrm{Ag} & 1.444139 & 3.909537 & 1.173933\end{array}$

$\begin{array}{llll}\text { Ag } & 2.879968 & 6.001216 & 2.135227\end{array}$

$\begin{array}{llll}\text { Ag } & -1.446051 & 3.908814 & 1.174028\end{array}$

$\begin{array}{llll}\mathrm{Ag} & -0.001480 & 6.081632 & 2.177362\end{array}$

$\begin{array}{llll}\mathrm{Ag} & 1.470653 & 6.246155 & -0.361521\end{array}$

$\begin{array}{llll}\mathrm{Ag} & -1.473877 & 6.245331 & -0.361356\end{array}$

$\begin{array}{llll}\mathrm{Ag} & 2.338604 & 2.393574 & -1.114238\end{array}$

$\begin{array}{llll}\mathrm{Ag} & 3.843124 & 4.523428 & -0.146823\end{array}$

$\begin{array}{llll}\mathrm{Ag} & 1.471408 & 4.259404 & 3.950581\end{array}$

$\begin{array}{llll}\mathrm{Ag} & 4.708683 & 0.531729 & 2.838725\end{array}$

$\begin{array}{llll}\mathrm{Ag} & 3.848608 & 3.326378 & 2.522928\end{array}$

$\begin{array}{llll}\mathrm{Ag} & 2.352615 & 1.222686 & 1.518485\end{array}$

$\begin{array}{llll}\mathrm{Ag} & 6.187994 & -0.553332 & -0.828002\end{array}$

$\begin{array}{llll}\mathrm{Ag} & 3.791192 & -1.001050 & 0.551977\end{array}$

$\begin{array}{llll}\mathrm{Ag} & 4.792868 & 1.746710 & 0.189574\end{array}$

$\begin{array}{llll}\mathrm{Ag} & 2.392426 & 1.470414 & 4.332156\end{array}$

$\begin{array}{llll}\text { Ag } & -0.000404 & 2.445205 & 5.637659\end{array}$

$\begin{array}{llll}\text { Ag } & -2.882788 & 5.999768 & 2.135461\end{array}$

$\begin{array}{llll}\mathrm{Ag} & -0.000469 & 2.117996 & 2.934150\end{array}$

$\begin{array}{llll}\text { Ag } & 3.841268 & -3.460304 & 2.058348\end{array}$

$\begin{array}{llll}\mathrm{Ag} & -4.682945 & -1.904711 & 4.279959\end{array}$

$\begin{array}{llll}\text { Ag } & -2.357671 & -1.165365 & 3.020337\end{array}$

$\begin{array}{llll}\mathrm{Ag} & 0.000726 & -2.815330 & 3.208326\end{array}$

$\begin{array}{llll}\mathrm{Ag} & -2.359368 & -3.578131 & 4.534733\end{array}$

$\begin{array}{llll}\mathrm{Ag} & -2.392759 & -1.044368 & 5.772932\end{array}$

$\begin{array}{llll}\mathrm{Ag} & 0.001412 & -5.160771 & 4.701152\end{array}$

$\begin{array}{llll}\text { Ag } \quad 2.361360 & -3.576993 & 4.534646\end{array}$

$\begin{array}{llll}\mathrm{Ag} & 0.000778 & -2.634424 & 5.968966\end{array}$

$\begin{array}{llll}\mathrm{Ag} & -3.850140 & 3.324429 & 2.523094\end{array}$

$\begin{array}{llll}\mathrm{Ag} & -2.392944 & 1.469137 & 4.332283\end{array}$

$\begin{array}{llll}\text { Ag } & -2.353099 & 1.221429 & 1.518645\end{array}$

$\begin{array}{llll}\text { Ag } & -2.339877 & 2.392342 & -1.114094\end{array}$

$\begin{array}{llll}\text { Ag } & -4.793705 & 1.744292 & 0.189847\end{array}$ 


\begin{tabular}{|c|c|c|c|}
\hline & & & \\
\hline & & & \\
\hline & -2.376147 & 690217 & \\
\hline & & & \\
\hline & 3.779338 & & \\
\hline & & & \\
\hline & 3.839446 & & \\
\hline & -3.7 & & \\
\hline & -2.8 & & \\
\hline & -5.2 & & \\
\hline & & & \\
\hline & -1.4 & -2 & \\
\hline & & & \\
\hline & -4.6 & & \\
\hline & 50 & & \\
\hline & -1.4 & & \\
\hline & & & \\
\hline & 0.0 & & \\
\hline & 1.4 & -5. & \\
\hline & 5.2 & & \\
\hline & 6.1 & & \\
\hline & 5.2 & & \\
\hline & 0.0 & -2.5 & \\
\hline & 2.8 & -4. & \\
\hline & -0.0 & & \\
\hline & & & \\
\hline & & -4. & \\
\hline & & -0.2 & \\
\hline & & & \\
\hline & 2.3 & & \\
\hline & 4.6 & & \\
\hline & 2.8 & -2. & \\
\hline & -0.0 & & \\
\hline & 1.4 & -0.7 & \\
\hline & -0.0 & & \\
\hline & -2.8 & -2 & 942 \\
\hline & & -1.1 & \\
\hline & 2.88 & -2.9 & -4 \\
\hline & -0.0 & & \\
\hline & -1.45 & -4.7 & 3763 \\
\hline & 0.0 & -3.0 & \\
\hline & & & \\
\hline & -1.42 & -1.182268 & -6.2 \\
\hline & -2.399173 & & \\
\hline & -1.427753 & -0.729881 & -3.520594 \\
\hline & 2.373532 & 4.691381 & -2.683018 \\
\hline
\end{tabular}




$$
\begin{array}{llll}
\mathrm{Ag} & -0.001722 & 6.325778 & -2.833834 \\
\mathrm{Ag} & -2.853258 & -2.500552 & -1.736912 \\
\mathrm{Ag} & -3.837539 & -0.284906 & -4.877047 \\
\mathrm{Ag} & -4.290421 & -4.664561 & -2.718122
\end{array}
$$

Ag92

$E=-13415.1001170$ Hartree

$\begin{array}{lrrr}\mathrm{Ag} & 4.724510 & -1.754822 & -3.573139 \\ \mathrm{Ag} & 5.605965 & -2.638742 & -1.002045 \\ \mathrm{Ag} & 2.959503 & -3.207179 & -0.190349 \\ \mathrm{Ag} & 1.081738 & -1.184816 & -0.464580 \\ \mathrm{Ag} & 4.661515 & -1.473118 & 1.452385 \\ \mathrm{Ag} & 3.757434 & -1.764673 & 4.249065 \\ \mathrm{Ag} & 1.494425 & -1.421605 & 6.092451 \\ \mathrm{Ag} & -3.568978 & 0.511934 & 4.720848 \\ \mathrm{Ag} & -2.925625 & -2.351153 & 4.604280 \\ \mathrm{Ag} & -3.422788 & -3.296736 & 1.850740 \\ \mathrm{Ag} & -2.503745 & -0.661752 & 2.435402 \\ \mathrm{Ag} & -1.310660 & -0.550040 & 6.273173 \\ \mathrm{Ag} & -0.275464 & -1.704870 & 3.948507 \\ \mathrm{Ag} & -0.648376 & -3.345699 & 6.074970 \\ \mathrm{Ag} & -5.132971 & -1.275317 & 2.993950 \\ \mathrm{Ag} & 0.100961 & -0.051219 & 1.814013 \\ \mathrm{Ag} & 1.956932 & -2.047940 & 2.148124 \\ \mathrm{Ag} & 1.151646 & -4.718184 & 1.556041 \\ \mathrm{Ag} & 1.601642 & -3.758002 & 4.312763 \\ \mathrm{Ag} & -1.576254 & -5.209087 & 0.659702 \\ \mathrm{Ag} & 2.161304 & -5.823353 & -0.900221 \\ \mathrm{Ag} & -3.289718 & -5.463610 & -1.575065 \\ \mathrm{Ag} & -1.127093 & -4.337456 & 3.426552 \\ \mathrm{Ag} & -0.581850 & -6.305163 & -1.749607 \\ \mathrm{Ag} & 0.251937 & -3.784169 & -1.090500 \\ \mathrm{Ag} & 3.986532 & -4.394304 & -2.574759 \\ \mathrm{Ag} & 3.823938 & -4.058735 & 2.417048 \\ \mathrm{Ag} & 4.794154 & -5.185038 & 0.019117 \\ \mathrm{Ag} & -0.733250 & -2.625542 & 1.282375 \\ \mathrm{Ag} & -5.159426 & -3.548305 & -0.428665 \\ \mathrm{Ag} & -2.470213 & -2.938190 & -0.915130 \\ \mathrm{Ag} & -1.607147 & -0.349222 & -0.291390 \\ \mathrm{Ag} & -2.405274 & -1.824028 & -4.858548 \\ \mathrm{Ag} & -4.251157 & -0.966377 & 0.274047 \\ \mathrm{Ag} & -6.842377 & -1.568628 & 0.768632 \\ \mathrm{Ag} & -6.187174 & 1.026237 & -0.242404 \\ \mathrm{Ag} & -6.039595 & -1.278469 & -1.928898 \\ \mathrm{Ag} & -5.268928 & 1.350789 & -2.929495 \\ \mathrm{Ag} & -4.470186 & 1.364659 & 2.040539\end{array}$




\begin{tabular}{|c|c|c|c|}
\hline g & -4.237365 & -3.240524 & -3.116471 \\
\hline$g$ & -5.098124 & -0.958498 & -4.591587 \\
\hline & -3.357590 & -0.654589 & -2.452034 \\
\hline & 1.267226 & -4.951149 & -3.471074 \\
\hline$g$ & 0.366424 & -2.685394 & -5.037111 \\
\hline & -0.645738 & -1.519553 & -2.693760 \\
\hline $\mathrm{Ag}$ & 3.086498 & -3.502010 & -5.118762 \\
\hline & 2.086528 & -2.346438 & -2.802703 \\
\hline $\mathrm{Ag}$ & -1.502621 & -4.138713 & -3.359 \\
\hline & -3.504027 & 1.662821 & -0.727745 \\
\hline Ag & -5.334984 & 3.639021 & -1.204456 \\
\hline $\mathrm{Ag}$ & -3.638425 & 3.968397 & 1.0 \\
\hline $\mathrm{Ag}$ & -0.947144 & 4.621860 & 1.936414 \\
\hline $\mathrm{Ag}$ & -0.082350 & 3.73 & \\
\hline $\mathrm{Ag}$ & 0.849635 & 1.447404 & 6.209311 \\
\hline $\mathrm{Ag}$ & -1.762032 & 1.95 & 1.4687 \\
\hline $\mathrm{Ag}$ & -1.909566 & 2.266730 & 6.303589 \\
\hline $\mathrm{Ag}$ & -2.812092 & 3.1606 & \\
\hline Ag & -0.908563 & 1.112488 & 4.063240 \\
\hline $\mathrm{Ag}$ & 0.931739 & 2.51 & \\
\hline $\mathrm{Ag}$ & 1.765355 & 5.102542 & 2.790189 \\
\hline $\mathrm{Ag}$ & 3.577791 & 0.561702 & 5.950162 \\
\hline $\mathrm{Ag}$ & 4.518862 & 0.8 & 3.259759 \\
\hline $\mathrm{Ag}$ & 1.846019 & 0.256534 & 3.885882 \\
\hline $\mathrm{Ag}$ & 2.716999 & 2.86 & 4.437633 \\
\hline $\mathrm{Ag}$ & 2.120007 & 6.717162 & 0.503809 \\
\hline $\mathrm{Ag}$ & 1.302374 & 4.16 & 0.109994 \\
\hline $\mathrm{Ag}$ & 3.459454 & 3.875390 & -3.343951 \\
\hline $\mathrm{Ag}$ & 4.133950 & 1.072428 & -3.526216 \\
\hline $\mathrm{Ag}$ & 3.090932 & 2.203175 & -1.079441 \\
\hline $\mathrm{Ag}$ & 2.486953 & -0.724922 & -5.099761 \\
\hline $\mathrm{Ag}$ & -0.278278 & 0.141213 & -5.000943 \\
\hline $\mathrm{Ag}$ & 1.849994 & 2.110037 & -4.984299 \\
\hline $\mathrm{Ag}$ & -0.903351 & 2.965719 & -4.806872 \\
\hline $\mathrm{Ag}$ & 1.478686 & 0.444506 & -2.756468 \\
\hline $\mathrm{Ag}$ & -3.037957 & 0.992098 & -4.743810 \\
\hline $\mathrm{Ag}$ & -3.184491 & 3.346819 & -3.054826 \\
\hline $\mathrm{Ag}$ & -1.279616 & 1.301716 & -2.578884 \\
\hline $\mathrm{Ag}$ & 0.835350 & 3.221973 & -2.575944 \\
\hline $\mathrm{Ag}$ & 1.205551 & 4.869479 & -4.777857 \\
\hline $\mathrm{Ag}$ & -1.029398 & 5.270408 & -3.054689 \\
\hline $\mathrm{Ag}$ & 1.682438 & 5.860711 & -2.157109 \\
\hline $\mathrm{Ag}$ & 0.463751 & 1.565327 & -0.352601 \\
\hline $\mathrm{Ag}$ & -3.249357 & 5.567197 & -1.265996 \\
\hline $\mathrm{Ag}$ & -1.407504 & 3.601116 & -0.789655 \\
\hline $\mathrm{Ag}$ & -0.548872 & 6.238944 & -0.408958 \\
\hline
\end{tabular}




$\begin{array}{lrrr}\mathrm{Ag} & 5.650117 & 2.801436 & -1.839318 \\ \mathrm{Ag} & 3.923515 & 4.849038 & -0.697137 \\ \mathrm{Ag} & 5.334261 & 1.180034 & 0.455573 \\ \mathrm{Ag} & 2.714085 & 0.561781 & 1.180545 \\ \mathrm{Ag} & 3.716447 & -0.581095 & -1.192768 \\ \mathrm{Ag} & 6.272459 & 0.031766 & -1.952010 \\ \mathrm{Ag} & 3.610159 & 3.205665 & 1.642987\end{array}$

Ag93

$\mathrm{E}=-13560.9076175$ Hartree

$\begin{array}{llll}\mathrm{Ag} & 1.352856 & -0.590499 & -0.661090\end{array}$

$\begin{array}{llll}\text { Ag } & 4.976515 & -2.343509 & -3.292818\end{array}$

$\begin{array}{llll}\mathrm{Ag} & -2.454805 & -0.511778 & 5.420458\end{array}$

$\begin{array}{llll}\mathrm{Ag} & 0.382024 & 0.282403 & 1.735023\end{array}$

$\begin{array}{llll}\mathrm{Ag} & -0.622112 & 1.163613 & 4.113007\end{array}$

$\begin{array}{llll}\mathrm{Ag} & 0.281241 & -0.127448 & 6.421234\end{array}$

$\begin{array}{llll}\text { Ag } & -1.614526 & 2.038053 & 6.481227\end{array}$

$\begin{array}{llll}\mathrm{Ag} & -0.516822 & -2.711029 & 5.268016\end{array}$

$\begin{array}{lllll}\mathrm{Ag} & 2.184466 & -2.272649 & 6.184405\end{array}$

$\begin{array}{llll}\mathrm{Ag} & 1.763649 & -3.769626 & 3.771837\end{array}$

$\begin{array}{llll}\mathrm{Ag} & 1.285083 & -1.000642 & 3.963596\end{array}$

$\begin{array}{lllll}\mathrm{Ag} & 6.590043 & -2.323522 & -0.954535\end{array}$

$\begin{array}{llll}\mathrm{Ag} & 5.649846 & -1.373708 & 1.603871\end{array}$

$\begin{array}{lllll}\mathrm{Ag} & 4.020873 & -1.413650 & -0.767563\end{array}$

$\begin{array}{llll}\mathrm{Ag} & 3.009914 & -0.526086 & 1.691182\end{array}$

$\begin{array}{llll}\mathrm{Ag} & 3.056681 & 0.411897 & 5.599246\end{array}$

$\begin{array}{llll}\mathrm{Ag} & 0.842831 & -2.428174 & 1.528059\end{array}$

$\begin{array}{llll}\mathrm{Ag} & 3.522583 & -3.318716 & 1.419087\end{array}$

$\begin{array}{llll}\mathrm{Ag} & 3.957158 & -1.843162 & 3.952528\end{array}$

$\begin{array}{llll}\mathrm{Ag} & 4.444185 & -4.193123 & -1.167027\end{array}$

Ag $\quad-5.774698 \quad-1.765916 \quad-1.616197$

$\begin{array}{llll}\mathrm{Ag} & -1.319175 & -1.107106 & 0.119498\end{array}$

$\begin{array}{llll}\mathrm{Ag} & -4.009834 & -0.323719 & 0.076571\end{array}$

$\begin{array}{llll}\mathrm{Ag} & -4.936404 & -4.255688 & -0.353322\end{array}$

$\begin{array}{lllll}\text { Ag } & -3.069547 & -2.530727 & -1.536381\end{array}$

$\begin{array}{llll}\mathrm{Ag} & -5.878466 & -2.065173 & 1.224407\end{array}$

$\begin{array}{llll}\mathrm{Ag} & -2.781797 & -5.580468 & 0.980531\end{array}$

$\begin{array}{llll}\mathrm{Ag} & -4.153461 & -0.610756 & 3.006801\end{array}$

$\begin{array}{llll}\mathrm{Ag} & -3.151300 & -2.801634 & 1.334560\end{array}$

$\begin{array}{llll}\text { Ag } & -4.957001 & -4.469511 & 2.468013\end{array}$

$\begin{array}{llll}\text { Ag } & -1.407838 & -1.379995 & 2.993736\end{array}$

$\begin{array}{llll}\mathrm{Ag} & -3.226803 & -3.066919 & 4.198222\end{array}$

$\begin{array}{llll}\mathrm{Ag} & 2.198619 & -6.021941 & -1.302141\end{array}$

$\begin{array}{llll}\mathrm{Ag} & -0.474788 & -6.541738 & -0.506374\end{array}$

$\begin{array}{llll}\mathrm{Ag} & -0.894776 & -3.858768 & -0.169157\end{array}$

$\begin{array}{llll}\mathrm{Ag} & -0.997035 & -4.191761 & 2.757822\end{array}$ 


\begin{tabular}{|c|c|c|c|}
\hline g & 1.283485 & -5.165884 & 1.231631 \\
\hline$g$ & -0.385906 & -2.037027 & -2.390674 \\
\hline & -4.055235 & -1.304221 & -3.901676 \\
\hline & 0.593673 & -2.933265 & -4.861046 \\
\hline$g$ & -2.146149 & -3.465835 & -4.050746 \\
\hline & -2.668463 & -5.293881 & -1.861343 \\
\hline $\mathrm{Ag}$ & -4.806587 & -3.945583 & -3.175650 \\
\hline & 0.045388 & -4.818805 & -2.723066 \\
\hline $\mathrm{Ag}$ & 1.800161 & -3.331584 & -0.964608 \\
\hline g & 2.321051 & -1.485833 & -3.123291 \\
\hline $\mathrm{Ag}$ & 2.770253 & -4.240158 & -3.476867 \\
\hline$g$ & 3.285421 & -2.374522 & -5.571223 \\
\hline $\mathrm{Ag}$ & 4.864235 & 1.173973 & 0.344224 \\
\hline $\mathrm{Ag}$ & 7.376431 & 0.0 & \\
\hline $\mathrm{Ag}$ & 5.812471 & 0.197329 & -2.245050 \\
\hline $\mathrm{Ag}$ & 3.137476 & 1.066 & -2.034305 \\
\hline $\mathrm{Ag}$ & 3.926108 & 3.418740 & 2.031130 \\
\hline $\mathrm{Ag}$ & 2.159741 & 1.960562 & 0.461947 \\
\hline $\mathrm{Ag}$ & -3.313608 & 1.990269 & 4.170611 \\
\hline $\mathrm{Ag}$ & 1.646975 & 4.455375 & 3.530304 \\
\hline Ag & 2.104364 & 1.693343 & 3.306367 \\
\hline $\mathrm{Ag}$ & 1.112047 & 2.614478 & 5.753120 \\
\hline $\mathrm{Ag}$ & -1.118956 & 711 & 4.346738 \\
\hline $\mathrm{Ag}$ & -0.091299 & 2.982399 & 1.936077 \\
\hline $\mathrm{Ag}$ & 3.812513 & 3.095267 & 4.876965 \\
\hline $\mathrm{Ag}$ & 4.797558 & 0.900896 & 3.276429 \\
\hline $\mathrm{Ag}$ & -1.343424 & -0.779926 & -4.783881 \\
\hline $\mathrm{Ag}$ & -5.830928 & 3.103097 & -0.663100 \\
\hline $\mathrm{Ag}$ & -4.146203 & 3.616504 & -2.924179 \\
\hline $\mathrm{Ag}$ & -0.526646 & 1.520508 & -0.519214 \\
\hline $\mathrm{Ag}$ & -1.459712 & 2.791508 & -2.830872 \\
\hline $\mathrm{Ag}$ & -2.385667 & 4.056216 & -5.121779 \\
\hline $\mathrm{Ag}$ & -3.235493 & 1.405274 & -4.560726 \\
\hline $\mathrm{Ag}$ & -4.929587 & 1.884579 & 1.723459 \\
\hline $\mathrm{Ag}$ & -2.257005 & 1.088300 & 1.770878 \\
\hline $\mathrm{Ag}$ & -3.205090 & 2.338670 & -0.569955 \\
\hline $\mathrm{Ag}$ & -2.301723 & 0.127503 & -2.242595 \\
\hline $\mathrm{Ag}$ & -5.019751 & 0.930525 & -2.323441 \\
\hline $\mathrm{Ag}$ & -6.632362 & 0.454426 & -0.020788 \\
\hline $\mathrm{Ag}$ & -3.711560 & 5.092668 & -0.515383 \\
\hline $\mathrm{Ag}$ & -2.802857 & 3.861707 & 1.919719 \\
\hline $\mathrm{Ag}$ & -0.583317 & 5.692534 & 2.072528 \\
\hline $\mathrm{Ag}$ & 1.409714 & -0.243897 & -5.514757 \\
\hline $\mathrm{Ag}$ & 4.106015 & 0.155301 & -4.543603 \\
\hline $\mathrm{Ag}$ & 4.842066 & 2.694845 & -3.417821 \\
\hline $\mathrm{Ag}$ & 0.289985 & 4.480732 & -4.242048 \\
\hline
\end{tabular}




$\begin{array}{lrrr}\mathrm{Ag} & 2.964313 & 4.820565 & -3.278882 \\ \mathrm{Ag} & 1.241816 & 3.205894 & -1.890675 \\ \mathrm{Ag} & -1.018587 & 4.247449 & -0.378631 \\ \mathrm{Ag} & 1.698414 & 4.748421 & 0.588521 \\ \mathrm{Ag} & 3.930537 & 3.631817 & -0.905632 \\ \mathrm{Ag} & 0.710078 & 5.953265 & -1.827934 \\ \mathrm{Ag} & -1.969491 & 5.546284 & -2.736865 \\ \mathrm{Ag} & -0.511052 & 1.933102 & -5.363832 \\ \mathrm{Ag} & 0.430474 & 0.656143 & -3.045501 \\ \mathrm{Ag} & 2.216846 & 2.337576 & -4.465870 \\ \mathrm{Ag} & -1.510329 & 6.907192 & -0.298642\end{array}$

Ag94

$\mathrm{E}=-13706.7307134$ Hartree

$\begin{array}{llll}\mathrm{Ag} & 0.860924 & 1.736410 & 5.639103\end{array}$

$\begin{array}{llll}\mathrm{Ag} & -4.016715 & 5.033297 & -0.773293\end{array}$

$\begin{array}{llll}\text { Ag } & -1.884565 & 5.848744 & 1.031269\end{array}$

$\begin{array}{llll}\mathrm{Ag} & -0.198237 & 1.764592 & 3.068136\end{array}$

$\begin{array}{llll}\mathrm{Ag} & -0.200963 & 4.177532 & 4.403415\end{array}$

$\begin{array}{llll}\text { Ag } & -1.908383 & 3.363478 & -0.254488\end{array}$

$\begin{array}{llll}\mathrm{Ag} & -5.512441 & 2.865310 & -1.868946\end{array}$

$\begin{array}{llll}\text { Ag } & -3.411972 & 1.172449 & -1.332218\end{array}$

$\begin{array}{llll}\mathrm{Ag} & -2.222916 & -0.423629 & -3.453843\end{array}$

$\begin{array}{llll}\mathrm{Ag} & -2.941484 & 3.530938 & -2.946296\end{array}$

$\begin{array}{llll}\text { Ag } & -2.400489 & 3.535588 & 2.660523\end{array}$

$\begin{array}{llll}\mathrm{Ag} & -2.022574 & 1.995929 & 5.153106\end{array}$

$\begin{array}{llll}\mathrm{Ag} & -2.348062 & 1.062668 & 1.296896\end{array}$

$\begin{array}{llll}\mathrm{Ag} & -4.541458 & 2.759543 & 0.831522\end{array}$

$\begin{array}{llll}\mathrm{Ag} & 0.856563 & -0.672198 & 4.197039\end{array}$

$\begin{array}{llll}\mathrm{Ag} & 1.890792 & -0.739356 & 6.704320\end{array}$

$\begin{array}{llll}\mathrm{Ag} & -3.217669 & -0.335413 & -6.047631\end{array}$

$\begin{array}{llll}\mathrm{Ag} & -4.663572 & -1.673241 & -4.006931\end{array}$

$\begin{array}{lllll}\mathrm{Ag} & -0.119686 & -5.341401 & -1.208758\end{array}$

$\begin{array}{llll}\mathrm{Ag} & -2.177719 & -2.778605 & -5.001837\end{array}$

Ag $\quad-1.168416 \quad-2.878633 \quad-2.349203$

Ag $\quad-3.618459 \quad-4.095549 \quad-2.910102$

$\begin{array}{llll}\mathrm{Ag} & -0.188264 & -0.607627 & 1.677823\end{array}$

$\begin{array}{llll}\mathrm{Ag} & 0.429632 & -2.999937 & 5.685251\end{array}$

$\begin{array}{llll}\text { Ag } & -0.624884 & -2.894888 & 3.099501\end{array}$

$\begin{array}{llll}\mathrm{Ag} & -0.160671 & -2.984314 & 0.278301\end{array}$

$\begin{array}{llll}\text { Ag } & -1.062749 & -5.166461 & 4.515862\end{array}$

$\begin{array}{llll}\mathrm{Ag} & 1.574191 & -4.591213 & 3.494404\end{array}$

$\begin{array}{llll}\mathrm{Ag} & -0.606377 & -5.330115 & 1.686714\end{array}$

$\begin{array}{llll}\mathrm{Ag} & -5.860051 & -0.071024 & -1.977547\end{array}$

$\begin{array}{llll}\mathrm{Ag} & -2.604596 & -1.854534 & 1.283880\end{array}$

$\begin{array}{llll}\text { Ag } & -6.921019 & 1.451765 & 0.084970\end{array}$ 


\begin{tabular}{|c|c|c|c|}
\hline $\mathrm{Ag}$ & -4.385979 & 1.272167 & -4.010047 \\
\hline $\mathrm{Ag}$ & -6.045469 & -2.973508 & -1.868602 \\
\hline & -4.469459 & -1.667940 & 3.353303 \\
\hline & -7.182633 & -1.370731 & 0.093592 \\
\hline g & -5.064888 & -3.071801 & 0.826601 \\
\hline & -3.763059 & -0.230764 & 5.754988 \\
\hline & -4.824750 & -0.167561 & 0.85 \\
\hline & -4.207632 & 1.273767 & 4335 \\
\hline g & -3.668938 & -1.750479 & 18944 \\
\hline & -1.213866 & -0.514084 & 50983 \\
\hline g & -1.985361 & -0.416886 & 6140 \\
\hline$g$ & -0.951247 & -0.477618 & 6.3 \\
\hline g & -2.635767 & -4.27 & -0.210484 \\
\hline $\mathrm{Ag}$ & -3.089861 & -4.18 & \\
\hline $\mathrm{Ag}$ & -2.459962 & -2.740512 & 5.199612 \\
\hline $\mathrm{g}$ & -0.421269 & -0.578792 & -5.6 \\
\hline $\lg$ & 0.596602 & -0.671967 & -3.047587 \\
\hline $\mathrm{g}$ & 0.66112 & -3.05 & -4.5 \\
\hline $\mathrm{Ag}$ & -1.094356 & -5.169736 & -3.853350 \\
\hline & 1.986 & -2.245 & \\
\hline $\mathrm{Ag}$ & 3.796274 & -2.402698 & -0.0 \\
\hline$g$ & 5.977042 & -3.9858 & 0.19 \\
\hline $\mathrm{Ag}$ & 4.189 & -3.87 & 2.3 \\
\hline $\mathrm{g}$ & 6.320006 & -1.14 & 0.15 \\
\hline $\mathrm{Ag}$ & 2.077939 & -4.68 & 0.5 \\
\hline $\lg$ & 3.913222 & -4.756496 & -1.626346 \\
\hline $\mathrm{Ag}$ & 1.642226 & -3.13 & 30994 \\
\hline $\mathrm{Ag}$ & 1.692650 & -5.423646 & -3.380681 \\
\hline $\mathrm{Ag}$ & 3.483712 & -3.285622 & -4.038805 \\
\hline $\mathrm{Ag}$ & 5.652026 & -2.568140 & -2.230218 \\
\hline $\mathrm{Ag}$ & 5.203244 & -1.088168 & -4.614479 \\
\hline $\mathrm{Ag}$ & 2.429854 & -0.833935 & -5.175096 \\
\hline $\mathrm{g}$ & 1.576033 & -0.765800 & -0.392546 \\
\hline $\mathrm{Ag}$ & 3.396458 & -0.925758 & -2.512354 \\
\hline $\mathrm{Ag}$ & 3.080616 & -2.332871 & 4.61 \\
\hline $\mathrm{Ag}$ & 4.533197 & -1.012354 & 2.407035 \\
\hline $\mathrm{Ag}$ & 6.478451 & 1.756933 & 0.172779 \\
\hline $\mathrm{Ag}$ & 2.091437 & 1.551944 & -1.952124 \\
\hline $\mathrm{Ag}$ & 3.916245 & 1.386643 & -4.078899 \\
\hline $\mathrm{Ag}$ & -1.769407 & 1.895558 & -5.043624 \\
\hline $\mathrm{Ag}$ & 5.896707 & 0.335798 & -2.246166 \\
\hline $\mathrm{Ag}$ & 1.084318 & 1.672381 & -4.626069 \\
\hline $\mathrm{Ag}$ & -0.281496 & 4.129547 & -3.931091 \\
\hline $\mathrm{Ag}$ & 4.063298 & 0.492657 & -0.084422 \\
\hline $\mathrm{Ag}$ & 2.728304 & 2.940704 & 0.532476 \\
\hline $\mathrm{Ag}$ & -0.760114 & 1.803735 & -2.426995 \\
\hline
\end{tabular}




$\begin{array}{lrrr}\mathrm{Ag} & 2.556155 & 3.877892 & -3.453558 \\ \mathrm{Ag} & 0.263174 & 1.651389 & 0.215357 \\ \mathrm{Ag} & 3.193800 & 5.283580 & -1.001390 \\ \mathrm{Ag} & 1.185785 & 6.268185 & -2.767052 \\ \mathrm{Ag} & 4.597132 & 2.849748 & -1.668922 \\ \mathrm{Ag} & 0.724521 & 3.967203 & -1.283852 \\ \mathrm{Ag} & -1.428090 & 5.704392 & -1.787683 \\ \mathrm{Ag} & 3.344051 & 0.581065 & 4.613749 \\ \mathrm{Ag} & 4.714754 & 1.892931 & 2.375744 \\ \mathrm{Ag} & 5.148532 & 4.191269 & 0.787384 \\ \mathrm{Ag} & 2.250021 & 0.624852 & 2.066105 \\ \mathrm{Ag} & 2.722768 & 5.431004 & 1.814069 \\ \mathrm{Ag} & 0.255495 & 4.076363 & 1.561873 \\ \mathrm{Ag} & 2.290949 & 3.099605 & 3.440897 \\ \mathrm{Ag} & 0.255202 & 6.469802 & 2.823750 \\ \mathrm{Ag} & 0.727378 & 6.441586 & 0.033415\end{array}$

Ag95

$\mathrm{E}=-13852.5459548$ Hartree

$\begin{array}{llll}\mathrm{Ag} & 4.233914 & -0.895834 & 3.028220\end{array}$

$\begin{array}{llll}\mathrm{Ag} & 6.224420 & 0.281624 & -1.444061\end{array}$

$\begin{array}{lllll}\mathrm{Ag} & 0.430937 & -0.792747 & -5.657162\end{array}$

$\begin{array}{lllll}\mathrm{Ag} & 2.418339 & 1.487692 & -1.724289\end{array}$

$\begin{array}{lllll}\mathrm{Ag} & 1.783227 & 1.503168 & -4.516177\end{array}$

$\begin{array}{llll}\text { Ag } & 1.693542 & -0.773154 & -0.162234\end{array}$

Ag $\quad 5.979779 \quad-2.621458 \quad-1.353237$

$\begin{array}{llll}\mathrm{Ag} & 6.312255 & -1.110272 & 1.051605\end{array}$

$\begin{array}{llll}\mathrm{Ag} & 4.515112 & 1.252714 & -3.572967\end{array}$

$\begin{array}{llll}\mathrm{Ag} & 5.863734 & -1.232969 & -3.831220\end{array}$

$\begin{array}{lllll}\mathrm{Ag} & 3.192191 & -1.014653 & -4.777960\end{array}$

$\begin{array}{llll}\mathrm{Ag} & 1.087442 & -0.783770 & -2.929779\end{array}$

$\begin{array}{lllll}\mathrm{Ag} & 3.786386 & -1.002162 & -2.004932\end{array}$

$\begin{array}{llll}\mathrm{Ag} & -0.994811 & 1.696303 & -5.322949\end{array}$

$\begin{array}{llll}\mathrm{Ag} & 4.111748 & 0.509842 & 0.438546\end{array}$

$\begin{array}{llll}\mathrm{Ag} & 4.859162 & 2.808823 & -1.147579\end{array}$

Ag $\quad-1.004089 \quad-0.554024 \quad-1.007842$

$\begin{array}{llll}\mathrm{Ag} & -2.495464 & -4.306477 & -0.432534\end{array}$

$\begin{array}{llll}\mathrm{Ag} & -3.350117 & -1.837571 & -1.779013\end{array}$

$\begin{array}{llll}\mathrm{Ag} & -5.044171 & -3.073088 & 0.225736\end{array}$

Ag $\quad-5.672796 \quad-3.038167 \quad-2.553955$

Ag $\quad-3.121070 \quad-4.224647 \quad-3.245753$

$\begin{array}{lllll}\mathrm{Ag} & 0.135092 & -5.389011 & -1.029843\end{array}$

$\begin{array}{llll}\mathrm{Ag} & 2.216627 & -5.536358 & -2.937228\end{array}$

Ag $\quad-0.753720 \quad-2.977816 \quad-2.397124$

$\begin{array}{llll}\text { Ag } & 1.964998 & -3.196402 & -1.540034\end{array}$

Ag $\quad \begin{array}{llll}1.356264 & -3.218411 & -4.360486\end{array}$ 


\begin{tabular}{lrrr}
$\mathrm{Ag}$ & -0.484580 & -5.319313 & -3.787602 \\
$\mathrm{Ag}$ & -1.406295 & -2.975706 & -5.160072 \\
$\mathrm{Ag}$ & -4.009479 & -1.834461 & -4.548599 \\
$\mathrm{Ag}$ & -1.646319 & -0.565250 & -3.730287 \\
$\mathrm{Ag}$ & -2.292816 & -0.578436 & -6.428677 \\
$\mathrm{Ag}$ & -3.354856 & -4.107766 & 2.403293 \\
$\mathrm{Ag}$ & 3.845356 & -2.384998 & 0.527769 \\
$\mathrm{Ag}$ & 4.175477 & -4.794894 & -0.915867 \\
$\mathrm{Ag}$ & 4.080891 & -3.415563 & -3.418744 \\
$\mathrm{Ag}$ & -2.662422 & -1.840982 & 0.964929 \\
$\mathrm{Ag}$ & 2.060893 & -4.653022 & 1.028213 \\
$\mathrm{Ag}$ & -0.750039 & -5.268567 & 1.770303 \\
$\mathrm{Ag}$ & -0.111931 & -2.977495 & 0.346364 \\
$\mathrm{Ag}$ & -1.598673 & -5.000422 & 4.501521 \\
$\mathrm{Ag}$ & 1.762015 & -2.156429 & 2.376225 \\
$\mathrm{Ag}$ & -0.965810 & -2.783051 & 3.074180 \\
$\mathrm{Ag}$ & 2.483064 & -2.138009 & 5.061539 \\
$\mathrm{Ag}$ & 3.900606 & -3.756785 & 3.058608 \\
$\mathrm{Ag}$ & 5.965891 & -3.948074 & 1.146753 \\
$\mathrm{Ag}$ & 1.156218 & -4.448256 & 3.834854 \\
$\mathrm{Ag}$ & -5.474327 & -0.168121 & -2.729338 \\
$\mathrm{Ag}$ & -2.408265 & 1.112995 & 0.905997 \\
$\mathrm{Ag}$ & -6.819521 & 1.417504 & -0.814799 \\
$\mathrm{Ag}$ & -3.798886 & 4.974969 & -1.527217 \\
$\mathrm{Ag}$ & -4.725711 & -0.174374 & 0.093647 \\
$\mathrm{Ag}$ & -4.528003 & 2.764789 & 0.087016 \\
$\mathrm{Ag}$ & -2.653146 & 2.170241 & 4.707663 \\
$\mathrm{Ag}$ & -4.600262 & 1.368869 & 2.640188 \\
$\mathrm{Ag}$ & -0.732265 & 4.337150 & 4.156373 \\
$\mathrm{Ag}$ & -2.665172 & 3.629446 & 2.152118 \\
$\mathrm{Ag}$ & -0.293493 & -2.781210 & 5.782872 \\
$\mathrm{Ag}$ & -0.539791 & 1.876703 & 2.926713 \\
$\mathrm{Ag}$ & -1.931870 & 5.877386 & 0.518654 \\
$\mathrm{Ag}$ & -0.060836 & 6.569877 & 2.566906 \\
$\mathrm{Ag}$ & -5.144247 & 2.740957 & -2.695494 \\
$\mathrm{Ag}$ & -3.089730 & 1.114169 & -1.868416 \\
$\mathrm{Ag}$ & -1.772070 & 3.348715 & -0.663391 \\
$\mathrm{Ag}$ & -2.442176 & 3.405744 & -3.476708 \\
$\mathrm{Ag}$ & -3.735182 & 1.091358 & -4.626670 \\
$\mathrm{Ag}$ & -7.077312 & -1.395463 & -0.738553 \\
$\mathrm{Ag}$ & -0.338413 & -0.547058 & 1.639307 \\
$\mathrm{Ag}$ & -2.456340 & -1.567460849 & 1.670677 \\
\hline
\end{tabular}




$\begin{array}{lrrr}\mathrm{Ag} & -4.473122 & -0.038967 & 5.136781 \\ \mathrm{Ag} & -1.759030 & -0.246598 & 6.102245 \\ \mathrm{Ag} & 0.335586 & -0.509564 & 4.278380 \\ \mathrm{Ag} & 2.523439 & 5.508848 & 1.945026 \\ \mathrm{Ag} & 1.870044 & 3.239307 & 3.587172 \\ \mathrm{Ag} & 0.313238 & 1.659448 & 0.166474 \\ \mathrm{Ag} & 0.114636 & 4.132201 & 1.408383 \\ \mathrm{Ag} & -1.093286 & 5.627711 & -2.204083 \\ \mathrm{Ag} & 3.378859 & 5.258913 & -0.771820 \\ \mathrm{Ag} & 1.631446 & 6.166220 & -2.833299 \\ \mathrm{Ag} & 0.973260 & 3.921403 & -1.341134 \\ \mathrm{Ag} & 3.081335 & 3.758001 & -3.235285 \\ \mathrm{Ag} & 0.333962 & 3.979151 & -4.106365 \\ \mathrm{Ag} & -0.336531 & 1.710362 & -2.596389 \\ \mathrm{Ag} & 0.792192 & 6.441288 & -0.130135 \\ \mathrm{Ag} & 5.068405 & 4.244240 & 1.309694 \\ \mathrm{Ag} & 2.020238 & 0.713347 & 2.313407 \\ \mathrm{Ag} & 2.740889 & 0.772362 & 4.988614 \\ \mathrm{Ag} & 4.416941 & 2.005893 & 2.911834 \\ \mathrm{Ag} & 6.467271 & 1.794684 & 0.977747 \\ \mathrm{Ag} & 1.005357 & -0.475538 & 6.905562 \\ \mathrm{Ag} & 2.706973 & 2.972932 & 0.772671\end{array}$

Ag96

$\mathrm{E}=-13998.3150149$ Hartree

$\begin{array}{lrrr}\mathrm{Ag} & 0.310413 & -5.200708 & -3.643124 \\ \mathrm{Ag} & 0.217892 & -1.421621 & -2.447487 \\ \mathrm{Ag} & -2.514069 & -5.178312 & -3.837760 \\ \mathrm{Ag} & -1.377840 & -0.000069 & -0.541007 \\ \mathrm{Ag} & -2.514509 & 5.178511 & -3.837177 \\ \mathrm{Ag} & -4.167642 & 3.793082 & -2.008263 \\ \mathrm{Ag} & -2.728472 & 6.064233 & -1.165150 \\ \mathrm{Ag} & -1.278693 & 3.760444 & -1.784957 \\ \mathrm{Ag} & -1.476714 & 4.709469 & 0.971943 \\ \mathrm{Ag} & -4.300657 & 4.658414 & 0.741979 \\ \mathrm{Ag} & -5.736890 & 2.330345 & -0.130654 \\ \mathrm{Ag} & -2.886702 & 2.345004 & 0.098503 \\ \mathrm{Ag} & -1.051599 & 2.863481 & -4.537251 \\ \mathrm{Ag} & -5.511555 & 1.432305 & -2.872228 \\ \mathrm{Ag} & -3.861932 & 2.857577 & -4.690176 \\ \mathrm{Ag} & -2.653523 & 1.432709 & -2.643227 \\ \mathrm{Ag} & -3.902856 & 0.000151 & -4.738633 \\ \mathrm{Ag} & -5.511403 & -1.432478 & -2.872497 \\ \mathrm{Ag} & -1.051189 & -2.863196 & -4.537474 \\ \mathrm{Ag} & -1.065021 & 0.000194 & -4.574013 \\ \mathrm{Ag} & -3.861601 & -2.857229 & -4.690537\end{array}$




\begin{tabular}{|c|c|c|c|}
\hline$g$ & -2.653391 & -1.432693 & -2.643334 \\
\hline & 5.746759 & 2.330838 & 0.754434 \\
\hline & 5.943809 & 1.431596 & -1.987115 \\
\hline & 7.204235 & 0.000332 & 0.102322 \\
\hline & 4.482938 & -3.792220 & -1.342585 \\
\hline & 5.943902 & -1.431006 & -1.987082 \\
\hline & 4.641636 & 0.000145 & -4.079787 \\
\hline & 4.392429 & 0.000159 & -0.102542 \\
\hline & 1.504114 & 0.000035 & -0.3 \\
\hline & 1.792875 & -2.862603 & -4.3 \\
\hline & 4.593903 & -2.8 & -4.0 \\
\hline & 1.594809 & -3.759784 & -1.5 \\
\hline & 3.085928 & 2295 & -2.20 \\
\hline & 0.309891 & 5.200971 & -3.6 \\
\hline & 1.792 & 2.86 & -4.3 \\
\hline & 3.130864 & 5.177714 & -3.40 \\
\hline & 0.102881 & 6.099566 & -0.9 \\
\hline & 0.217 & 1.4 & -2.4 \\
\hline & 0.003893 & 2.345125 & 0.3 \\
\hline & 2.893 & 2.3 & \\
\hline & 3.085696 & 1.432512 & -2.200249 \\
\hline & 4.593 & 2.85 & -4.0 \\
\hline & 2.929756 & 6.065283 & -0.732573 \\
\hline & & 3.792621 & -1.3 \\
\hline $\mathrm{g}$ & 1.594 & 3.75 & -1.5 \\
\hline & 1.365 & 4.707749 & 1.1 \\
\hline$g$ & 4.195 & 4.66 & $1.3 \xi$ \\
\hline & 1.812167 & 0.000169 & -4.35 \\
\hline $\mathrm{Ag}$ & -4.267821 & -0.000202 & -0.7 \\
\hline & 2.893315 & -2.344245 & 0.54 \\
\hline $\mathrm{Ag}$ & -1.476252 & -4.709655 & 0.9 \\
\hline$g$ & -4.517003 & -2.352339 & 2.45 \\
\hline $\mathrm{Ag}$ & -4.167317 & -3.793128 & -2.008704 \\
\hline $\mathrm{g}$ & 0.004049 & -2.345152 & 0.31 \\
\hline$g$ & -2.886380 & -2.345252 & 0.09 \\
\hline $\mathrm{Ag}$ & -3.073787 & -4.620406 & 3.306615 \\
\hline$g$ & -5.736692 & -2.330961 & -0.130941 \\
\hline $\mathrm{Ag}$ & -7.078017 & -0.000307 & -1.003837 \\
\hline $\mathrm{Ag}$ & -1.278380 & -3.760452 & -1.785175 \\
\hline $\mathrm{Ag}$ & -4.300286 & -4.658877 & 0.741438 \\
\hline $\mathrm{Ag}$ & -2.727868 & -6.064366 & -1.165916 \\
\hline $\mathrm{Ag}$ & 2.930377 & -6.064998 & -0.732545 \\
\hline $\mathrm{Ag}$ & 0.103479 & -6.099433 & -0.978011 \\
\hline Ag & 3.131250 & -5.177456 & -3.404180 \\
\hline $\mathrm{Ag}$ & 5.746934 & -2.330300 & 0.754480 \\
\hline$\Delta \sigma$ & -1.626306 & -2.339712 & 2.652333 \\
\hline
\end{tabular}




$\begin{array}{lrrr}\mathrm{Ag} & -0.243681 & -4.641484 & 3.560112 \\ \mathrm{Ag} & 1.366237 & -4.707689 & 1.187132 \\ \mathrm{Ag} & 2.591353 & -4.627730 & 3.730617 \\ \mathrm{Ag} & 4.195740 & -4.659776 & 1.389745 \\ \mathrm{Ag} & 4.146942 & -2.351654 & 3.119897 \\ \mathrm{Ag} & 1.252497 & -2.334130 & 2.877601 \\ \mathrm{Ag} & 5.622001 & 0.000212 & 2.447014 \\ \mathrm{Ag} & -4.592422 & -0.000482 & 4.125233 \\ \mathrm{Ag} & 2.770269 & 0.000049 & 2.245857 \\ \mathrm{Ag} & -0.115544 & -0.000034 & 2.006238 \\ \mathrm{Ag} & -3.027096 & -0.000240 & 1.789360 \\ \mathrm{Ag} & -5.875674 & -0.000312 & 1.553253 \\ \mathrm{Ag} & -3.253969 & -0.000566 & 6.558771 \\ \mathrm{Ag} & -3.210333 & 2.327199 & 4.991359 \\ \mathrm{Ag} & -0.379408 & 2.360200 & 5.215257 \\ \mathrm{Ag} & -4.517162 & 2.351689 & 2.454590 \\ \mathrm{Ag} & -1.626561 & 2.339400 & 2.652449 \\ \mathrm{Ag} & 1.252299 & 2.334137 & 2.877633 \\ \mathrm{Ag} & -3.074171 & 4.619892 & 3.307117 \\ \mathrm{Ag} & 2.591008 & 4.627886 & 3.730714 \\ \mathrm{Ag} & -0.243999 & 4.641266 & 3.560269 \\ \mathrm{Ag} & -0.379265 & -2.360432 & 5.215160 \\ \mathrm{Ag} & 2.463301 & 2.352743 & 5.387888 \\ \mathrm{Ag} & 3.945304 & 0.000155 & 4.775507 \\ \mathrm{Ag} & 2.463521 & -2.352616 & 5.387893 \\ \mathrm{Ag} & 1.120483 & -0.000072 & 4.607532 \\ \mathrm{Ag} & 4.146815 & 2.352007 & 3.119830 \\ \mathrm{Ag} & -3.210090 & -2.328081 & 4.991144 \\ \mathrm{Ag} & -1.711157 & -0.000295 & 4.328730\end{array}$

Ag97

$E=-14144.1295435$ Hartree

$\begin{array}{lrrr}\mathrm{Ag} & -7.179667 & -1.922536 & 1.195355 \\ \mathrm{Ag} & -4.111138 & 5.827926 & -0.489892 \\ \mathrm{Ag} & -5.361531 & 1.339055 & -2.006308 \\ \mathrm{Ag} & -5.193492 & 3.286732 & 0.082960 \\ \mathrm{Ag} & -6.242227 & 0.673359 & 0.652786 \\ \mathrm{Ag} & -4.352800 & 1.933186 & -4.606369 \\ \mathrm{Ag} & -0.813373 & -0.678749 & -2.522970 \\ \mathrm{Ag} & -2.095279 & 3.681140 & -4.412027 \\ \mathrm{Ag} & -3.487754 & 0.999200 & 0.123969 \\ \mathrm{Ag} & -4.256695 & 3.911443 & -2.571177 \\ \mathrm{Ag} & -0.393171 & 1.404700 & -4.433992 \\ \mathrm{Ag} & -2.542831 & 1.617070 & -2.530851 \\ \mathrm{Ag} & -3.606112 & -0.956132 & -1.977136 \\ \mathrm{Ag} & -0.972533 & -2.603639 & -4.604099\end{array}$




\begin{tabular}{|c|c|c|c|}
\hline $\mathrm{g}$ & -6.369162 & -1.267747 & -1.429018 \\
\hline g & -1.112050 & -6.190361 & 0.751160 \\
\hline & -5.538121 & -4.135282 & 1.214822 \\
\hline & -1.728561 & -1.331506 & 0.148148 \\
\hline & 0.713492 & -4.853077 & -4.507596 \\
\hline & -2.039819 & -5.147164 & -3.981496 \\
\hline g & -2.810587 & -3.929710 & 0.715662 \\
\hline & -2.956740 & -5.792421 & -1.38 \\
\hline g & -0.182811 & -5.603412 & -1.903698 \\
\hline & 2.603690 & -5.290146 & -2.433935 \\
\hline $\mathrm{Ag}$ & -4.489994 & -1.603026 & 0.671960 \\
\hline g & -4.699384 & -3.553097 & -1.42 \\
\hline $\lg$ & -1.887922 & -3.262997 & -1.937 \\
\hline$g$ & -3.7556 & -2.896494 & -4.0 \\
\hline$g$ & -2.676302 & -0.322500 & -4.639077 \\
\hline $\mathrm{Ag}$ & -5.429427 & -0.612743 & -4.04 \\
\hline$g$ & 3.028148 & -3.187642 & -4.308149 \\
\hline g & 0.8917 & 2033 & -2.46 \\
\hline $\lg$ & 1.332603 & -0.908655 & -4.398571 \\
\hline $\mathrm{Ag}$ & 0.209036 & 48578 & \\
\hline $\mathrm{Ag}$ & -1.973833 & 5.626199 & -2.33 \\
\hline$g$ & 1.900599 & 3.097637 & -4.153260 \\
\hline $\mathrm{Ag}$ & -0.259377 & 3.327383 & -2.288342 \\
\hline $\mathrm{Ag}$ & -2.422874 & 3.571665 & -0.443269 \\
\hline $\mathrm{Ag}$ & 0.692489 & 5.873434 & -1.35 \\
\hline g & 2.846409 & 5.569817 & -3.163113 \\
\hline $\mathrm{Ag}$ & -0.687573 & 1.248710 & -0.39 \\
\hline $\lg$ & 3.606877 & 0.809867 & -4.118146 \\
\hline Ag & 4.894734 & -3.588354 & -2.15 \\
\hline $\mathrm{Ag}$ & 3.182235 & -1.289080 & -2.218 \\
\hline $\lg$ & 4.557989 & 3.314312 & -3.149115 \\
\hline $\mathrm{Ag}$ & 1.454686 & 1.014037 & -2.280530 \\
\hline $\mathrm{Ag}$ & 5.280649 & -1.452101 & -4.019772 \\
\hline $\mathrm{Ag}$ & 1.041883 & -1.069055 & -0.362111 \\
\hline $\mathrm{Ag}$ & -2.188918 & -1.592994 & 5.023262 \\
\hline $\mathrm{Ag}$ & -3.901695 & -2.889400 & 3.163487 \\
\hline $\mathrm{Ag}$ & -2.767832 & 3.284804 & 4.435773 \\
\hline $\mathrm{Ag}$ & -1.757567 & 2.303529 & 2.034843 \\
\hline $\mathrm{Ag}$ & -2.811188 & -0.289858 & 2.590040 \\
\hline $\mathrm{Ag}$ & -3.857598 & 0.708476 & 4.964758 \\
\hline $\mathrm{Ag}$ & -5.573057 & -0.603395 & 3.104623 \\
\hline $\mathrm{Ag}$ & -4.561058 & 2.017777 & 2.570844 \\
\hline $\mathrm{Ag}$ & -3.467615 & 4.594068 & 1.977587 \\
\hline $\mathrm{Ag}$ & 0.628527 & -4.943206 & 2.673197 \\
\hline $\mathrm{Ag}$ & -1.090392 & -2.605008 & 2.619983 \\
\hline $\mathrm{Ag}$ & -2.154673 & -5.129420 & 3.175988 \\
\hline
\end{tabular}




$\begin{array}{rrrr}\mathrm{Ag} & 1.693594 & -5.960365 & 0.221709 \\ \mathrm{Ag} & -0.023627 & -0.021328 & 2.075370 \\ \mathrm{Ag} & -0.807079 & 4.832698 & 2.979506 \\ \mathrm{Ag} & -1.473694 & 6.063202 & 0.489958 \\ \mathrm{Ag} & 0.248132 & 3.777085 & 0.537006 \\ \mathrm{Ag} & 1.222722 & 6.216449 & 1.459672 \\ \mathrm{Ag} & 0.917388 & 2.495736 & 2.990558 \\ \mathrm{Ag} & 3.347452 & 5.993832 & -0.417835 \\ \mathrm{Ag} & 1.852681 & 4.948662 & 3.921155 \\ \mathrm{Ag} & -1.079551 & 1.008823 & 4.528923 \\ \mathrm{Ag} & -0.455654 & -3.845454 & 5.033156 \\ \mathrm{Ag} & 1.622066 & 1.255726 & 5.443299 \\ \mathrm{Ag} & -0.096955 & 3.518584 & 5.374983 \\ \mathrm{Ag} & 5.073949 & 3.739851 & -0.409396 \\ \mathrm{Ag} & 2.956449 & 3.970533 & 1.510118 \\ \mathrm{Ag} & 2.644458 & 0.178365 & 3.025008 \\ \mathrm{Ag} & 3.565626 & 2.681990 & 3.993245 \\ \mathrm{Ag} & 4.121171 & 1.218550 & -1.287309 \\ \mathrm{Ag} & 6.231389 & 1.030836 & -3.093566 \\ \mathrm{Ag} & 2.398295 & 3.529569 & -1.322558 \\ \mathrm{Ag} & 3.300111 & -1.038302 & 5.443756 \\ \mathrm{Ag} & 5.248941 & 0.392219 & 3.989805 \\ \mathrm{Ag} & 1.982609 & 1.450580 & 0.566479 \\ \mathrm{Ag} & 6.740649 & 1.442324 & -0.348204 \\ \mathrm{Ag} & 4.661897 & 1.682042 & 1.544666 \\ \mathrm{Ag} & 6.319089 & -0.621581 & 1.563008 \\ \mathrm{Ag} & 3.716523 & -0.876940 & 0.607203 \\ \mathrm{Ag} & 5.880535 & -1.085211 & -1.250040 \\ \mathrm{Ag} & 4.447850 & -5.659611 & -0.316863 \\ \mathrm{Ag} & -0.014133 & -3.672255 & 0.194753 \\ \mathrm{Ag} & 5.414137 & -3.180233 & 0.628851 \\ \mathrm{Ag} & 2.767125 & -3.397165 & -0.337996 \\ \mathrm{Ag} & 3.416476 & -4.642216 & 2.115950 \\ \mathrm{Ag} & 4.390405 & -2.141150 & 3.084039 \\ \mathrm{Ag} & 0.627175 & -1.279383 & 4.561145 \\ \mathrm{Ag} & 1.707080 & -2.348627 & 2.104178 \\ \mathrm{Ag} & 2.329260 & -3.549002 & 4.537824\end{array}$

\begin{tabular}{|c|c|c|c|}
\hline \\
\hline \multicolumn{4}{|c|}{$E=-14289.9875923$ Hartree } \\
\hline $\mathrm{Ag}$ & 5.238825 & -4.692273 & 0.545066 \\
\hline $\mathrm{Ag}$ & 2.327155 & 0.000014 & 0.580291 \\
\hline $\mathrm{Ag}$ & 6.130285 & -2.401030 & 2.014270 \\
\hline $\mathrm{Ag}$ & 7.080746 & 0.000048 & 0.731062 \\
\hline $\mathrm{Ag}$ & 6.130288 & 2.400954 & 2.014361 \\
\hline $\mathrm{Ag}$ & 4.664124 & -0.000021 & 2.159492 \\
\hline
\end{tabular}




\begin{tabular}{|c|c|c|c|}
\hline & 0.294764 & -2.862824 & -2.905081 \\
\hline g & -1.151162 & -5.255258 & -3.150680 \\
\hline & 4.059817 & -3.848565 & -1.895399 \\
\hline$\sigma_{0}$ & 0.100208 & -1.416779 & -0.508437 \\
\hline & 0.490192 & -4.299563 & -5.272223 \\
\hline & 2.796360 & 0.000145 & -4.361377 \\
\hline & -1.908306 & -2.898371 & -4.681419 \\
\hline & 0.498671 & -1.455142 & -5.364033 \\
\hline & -2.107022 & -1.443475 & -2.279255 \\
\hline & 0.300415 & 0.000102 & -2.972564 \\
\hline $\mathrm{Ag}$ & -1.932719 & 0.000099 & -4.755723 \\
\hline & 2.759069 & 2.898249 & -4.292205 \\
\hline & 0.498615 & 1.455420 & -5.3 \\
\hline & -4.323557 & -1.441410 & -3.995246 \\
\hline $\mathrm{Ag}$ & 2.759078 & -2.897974 & -4.29 \\
\hline g & 4.059752 & 3.848682 & -1.895171 \\
\hline $\mathrm{Ag}$ & 6.282252 & 2.3648 & -0.795042 \\
\hline $\mathrm{Ag}$ & 2.561460 & 1.442290 & -1.8 \\
\hline $\mathrm{Ag}$ & 5.028525 & -1.441333 & -3.215951 \\
\hline $\mathrm{Ag}$ & 5.028527 & 1.441533 & -3.2 \\
\hline $\mathrm{Ag}$ & 2.561436 & -1.442191 & -1.888400 \\
\hline $\mathrm{Ag}$ & 4.758613 & 0.00 & -0.76 \\
\hline $\mathrm{Ag}$ & 3.786023 & -2.364343 & 0.576042 \\
\hline $\mathrm{Ag}$ & 6.282304 & -2.364837 & -0.795 \\
\hline $\mathrm{Ag}$ & 7.202418 & 0.000025 & -2.09 \\
\hline $\lg$ & 1.755266 & -5.257464 & -2.910047 \\
\hline $\mathrm{Ag}$ & 1.549163 & -3.8 & -0.474819 \\
\hline $\mathrm{Ag}$ & -1.348872 & -3.798471 & -0.717554 \\
\hline $\mathrm{Ag}$ & -2.999308 & -4.709123 & 1.491663 \\
\hline $\mathrm{Ag}$ & -1.762273 & -3.848772 & 4.016425 \\
\hline $\mathrm{Ag}$ & 3.011004 & -6.111973 & -0.488112 \\
\hline $\mathrm{Ag}$ & 0.112679 & -6.196708 & -0.765794 \\
\hline $\mathrm{Ag}$ & -0.101030 & -4.802546 & 1.746945 \\
\hline $\mathrm{Ag}$ & -6.284540 & -2.394172 & 0.986936 \\
\hline $\mathrm{Ag}$ & -2.791982 & -6.104653 & -0.964480 \\
\hline $\mathrm{Ag}$ & -3.731097 & -2.358244 & -0.048573 \\
\hline $\mathrm{Ag}$ & -1.525546 & -2.351544 & 1.699552 \\
\hline $\mathrm{Ag}$ & -3.591149 & -3.845082 & -2.530827 \\
\hline $\mathrm{Ag}$ & 3.639097 & -2.431716 & 3.493746 \\
\hline $\mathrm{Ag}$ & 1.325957 & -2.354198 & 1.945298 \\
\hline $\mathrm{Ag}$ & 1.151489 & -3.852468 & 4.266016 \\
\hline $\mathrm{Ag}$ & 2.796150 & -4.725424 & 1.971426 \\
\hline $\mathrm{Ag}$ & -5.164574 & -4.685811 & -0.323370 \\
\hline $\mathrm{Ag}$ & -4.459763 & 0.000033 & -1.538006 \\
\hline $\mathrm{Ag}$ & -0.300238 & 1.441621 & 4.121701 \\
\hline $\mathrm{Ag}$ & 3.639109 & 2.431552 & 3.493790 \\
\hline
\end{tabular}




\begin{tabular}{|c|c|c|c|}
\hline $\mathrm{g}$ & -0.077672 & -0.000081 & \\
\hline Ag & -7.001848 & -0.000012 & -0.444814 \\
\hline & -2.267334 & -0.000022 & 0.182200 \\
\hline $\mathrm{Ag}$ & -4.091545 & -2.393160 & 2.875766 \\
\hline & -4.851653 & -0.000075 & 1.370096 \\
\hline g & -4.091605 & 2.392936 & 2.875836 \\
\hline$g$ & -4.996861 & -0.000124 & 4.227846 \\
\hline g & -2.556658 & -0.000045 & 3.017796 \\
\hline g & -6.284622 & 2.394063 & 0.987 \\
\hline & -0.515630 & -0.000149 & 6.5 \\
\hline $\mathrm{Ag}$ & -0.300207 & -1.441813 & \\
\hline$g$ & -2.800955 & 1.471020 & 5.376 \\
\hline $\mathrm{Ag}$ & -2.800943 & -1.471297 & \\
\hline$g$ & -0.515072 & 2.884532 & 6.4 \\
\hline $\mathrm{Ag}$ & -1.762338 & 3.84 & \\
\hline$g$ & 1.894552 & 1.492583 & 5.78 \\
\hline g & 2.181178 & -0.000088 & \\
\hline g & -0.515041 & -2.884869 & 6.4 \\
\hline $\mathrm{Ag}$ & 1.894519 & -1.492772 & 5.78 \\
\hline $\mathrm{Ag}$ & -5.965291 & -2.361390 & -1.8 \\
\hline $\mathrm{Ag}$ & 0.294676 & 2.863058 & -2.904926 \\
\hline $\mathrm{Ag}$ & 0.490176 & 4.299889 & -5.272066 \\
\hline $\mathrm{g}$ & 0.100227 & 1.416830 & -0.508436 \\
\hline $\mathrm{Ag}$ & -1.348890 & 3.798514 & -0.71 \\
\hline $\mathrm{Ag}$ & -1.525530 & 2.351399 & 1.699694 \\
\hline $\mathrm{Ag}$ & -1.151242 & 5.255366 & $-3.15 c$ \\
\hline $\mathrm{Ag}$ & -2.792041 & 6.104661 & -0.9 \\
\hline $\mathrm{Ag}$ & -5.164656 & 4.685683 & -0.32 \\
\hline $\mathrm{Ag}$ & -2.999251 & 4.709009 & 1.491835 \\
\hline $\mathrm{Ag}$ & -0.101056 & 4.802426 & 1.7 \\
\hline $\mathrm{Ag}$ & 1.549115 & 3.802650 & -0.474627 \\
\hline $\mathrm{Ag}$ & 2.796032 & 4.725359 & 1.971622 \\
\hline $\mathrm{Ag}$ & 0.112599 & 6.196762 & -0.765558 \\
\hline $\mathrm{Ag}$ & 1.755203 & 5.257635 & -2.909807 \\
\hline $\mathrm{Ag}$ & 3.010909 & 6.112052 & -0.487859 \\
\hline $\mathrm{Ag}$ & 3.786005 & 2.364376 & 0.576150 \\
\hline $\mathrm{Ag}$ & 1.326000 & 2.354137 & 1.945368 \\
\hline $\mathrm{Ag}$ & 5.238812 & 4.692249 & 0.545385 \\
\hline $\mathrm{Ag}$ & 1.151435 & 3.852331 & 4.266162 \\
\hline $\mathrm{Ag}$ & -3.731134 & 2.358254 & -0.048448 \\
\hline $\mathrm{Ag}$ & -3.591250 & 3.845204 & -2.530688 \\
\hline $\mathrm{Ag}$ & -6.653516 & 0.000084 & -3.248006 \\
\hline $\mathrm{Ag}$ & -2.107099 & 1.443583 & -2.279189 \\
\hline $\mathrm{Ag}$ & -4.323594 & 1.441567 & -3.995207 \\
\hline $\mathrm{Ag}$ & -5.965290 & 2.361415 & -1.813745 \\
\hline $\mathrm{Ag}$ & -1.908309 & 2.898527 & -4.681289 \\
\hline
\end{tabular}




\begin{tabular}{|c|c|c|c|}
\hline \multicolumn{4}{|c|}{ Ag99 } \\
\hline \multicolumn{4}{|c|}{$E=-14435.8213746$ Hartree } \\
\hline $\mathrm{Ag}$ & -3.885716 & & 3.163381 \\
\hline $\mathrm{Ag}$ & -2.348044 & -0.000304 & \\
\hline $\mathrm{Ag}$ & & & \\
\hline $\mathrm{Ag}$ & -4.669980 & & \\
\hline $\mathrm{Ag}$ & & & \\
\hline $\mathrm{Ag}$ & -3.885765 & & \\
\hline $\mathrm{Ag}$ & 1.462 & -3.8 & \\
\hline $\mathrm{Ag}$ & & & \\
\hline $\mathrm{Ag}$ & -1.4 & & \\
\hline $\mathrm{Ag}$ & & & \\
\hline $\mathrm{Ag}$ & -0.0 & & \\
\hline $\mathrm{Ag}$ & & & \\
\hline $\lg$ & & & \\
\hline $\lg$ & $-0 .($ & & \\
\hline Ag & $-0 .($ & & \\
\hline $\mathrm{Ag}$ & $-0 .($ & & \\
\hline $\lg$ & & & \\
\hline $\lg$ & & & \\
\hline $\mathrm{Ag}$ & & & \\
\hline $\lg$ & 3.8 & & \\
\hline $\mathrm{Ag}$ & -2.3 & & \\
\hline$g$ & $-0 .($ & & \\
\hline $\mathrm{Ag}$ & & & \\
\hline $\mathrm{Ag}$ & & & \\
\hline$g$ & & & \\
\hline $\lg$ & & & \\
\hline $\mathrm{Ag}$ & -0.0 & & \\
\hline $\mathrm{Ag}$ & $-0 .($ & & \\
\hline $\mathrm{Ag}$ & & & -0 . \\
\hline $\mathrm{Ag}$ & & & \\
\hline $\lg$ & & & \\
\hline $\lg$ & & & \\
\hline & & & \\
\hline $\lg$ & & & \\
\hline $\mathrm{Ag}$ & & & -2.2 \\
\hline $\mathrm{Ag}$ & 5.2 & & \\
\hline $\mathrm{Ag}$ & -0.000004 & -4.8 & \\
\hline $\mathrm{Ag}$ & & & \\
\hline $\mathrm{Ag}$ & 2.912783 & -4.711554 & 1.691091 \\
\hline $\mathrm{Ag}$ & & -2.3 & 1.773838 \\
\hline $\mathrm{Ag}$ & -1.431342 & & 1.773766 \\
\hline & 6.229847 & -2.395308 & 1.461480 \\
\hline $\mathrm{Ag}$ & 4.694857 & & -3.669181 \\
\hline
\end{tabular}




\begin{tabular}{|c|c|c|c|}
\hline $\mathrm{g}$ & 4.627353 & 0.000188 & -1.209224 \\
\hline$g$ & 6.146946 & 2.362859 & -1.356403 \\
\hline & 6.229742 & 2.395214 & 1.461981 \\
\hline $\mathrm{Ag}$ & 6.147033 & -2.362363 & -1.356829 \\
\hline & 2.306301 & -0.000026 & 0.318586 \\
\hline g & 6.954438 & 0.000402 & -2.727997 \\
\hline & 7.065680 & 0.000101 & 0.095605 \\
\hline g & -4.694793 & -1.441439 & -3.669461 \\
\hline$g$ & -2.344089 & 1.444376 & -2.145 \\
\hline & -4.627333 & 0.000097 & -1.209276 \\
\hline $\mathrm{Ag}$ & -6.954448 & 0.000319 & -2.728116 \\
\hline & -4.694797 & 1.442054 & -3.669185 \\
\hline $\mathrm{Ag}$ & -2.342932 & 2.899912 & -4.554 \\
\hline g & -1.459522 & 5.257008 & -3.090500 \\
\hline$g$ & 0.000017 & -2.864098 & -2.969 \\
\hline$g$ & 1.459564 & -5.256428 & -3.091425 \\
\hline $\mathrm{Ag}$ & -1.459454 & -5.256462 & -3.09 \\
\hline g & -2.342918 & -2.899098 & -4.555170 \\
\hline$g$ & 0.000067 & -6.196112 & -0.820119 \\
\hline $\mathrm{Ag}$ & -2.344050 & -1.44 & -2.14 \\
\hline $\lg$ & 0.000018 & -1.419045 & -0.569211 \\
\hline $\mathrm{Ag}$ & -2.374089 & 0.00 & -4.62 \\
\hline $\lg$ & -6.146953 & -2.362487 & -1.356854 \\
\hline $\mathrm{Ag}$ & -3.773764 & -2.360481 & 0.215470 \\
\hline $\mathrm{Ag}$ & -2.912628 & -6.106189 & -0.775633 \\
\hline $\mathrm{Ag}$ & & -3.800674 & -0.65 \\
\hline $\mathrm{Ag}$ & -3.840751 & -3.846494 & -2.270806 \\
\hline $\lg$ & -5.222773 & -4.687667 & 0.061805 \\
\hline $\mathrm{Ag}$ & -2.912702 & -4.711614 & 1.691055 \\
\hline $\mathrm{Ag}$ & 0.000080 & -1.455640 & -5.435278 \\
\hline $\mathrm{Ag}$ & 0.000042 & -4.300921 & -5.341996 \\
\hline $\mathrm{Ag}$ & -0.000015 & 0.000306 & -3.037504 \\
\hline $\mathrm{Ag}$ & 2.342941 & -2.899041 & -4.555211 \\
\hline $\mathrm{Ag}$ & 4.694861 & -1.441360 & -3.669457 \\
\hline $\mathrm{Ag}$ & 2.344073 & 1.4443 & -2.145986 \\
\hline $\mathrm{Ag}$ & -0.000037 & 1.419057 & -0.568956 \\
\hline $\mathrm{Ag}$ & 2.342927 & 2.899968 & -4.554655 \\
\hline $\mathrm{Ag}$ & 0.000026 & 2.864634 & -2.968608 \\
\hline $\mathrm{Ag}$ & 1.459496 & 5.257042 & -3.090496 \\
\hline $\mathrm{Ag}$ & -0.000013 & 1.456596 & -5.435053 \\
\hline $\mathrm{Ag}$ & 0.000024 & 4.301885 & -5.341238 \\
\hline $\mathrm{Ag}$ & 2.344067 & -1.443866 & -2.146298 \\
\hline $\mathrm{Ag}$ & 2.374130 & 0.000469 & -4.627613 \\
\hline $\mathrm{Ag}$ & -7.065683 & 0.000089 & 0.095549 \\
\hline $\mathrm{Ag}$ & -6.146964 & 2.362786 & -1.356471 \\
\hline $\mathrm{Ag}$ & -3.840651 & 3.846912 & -2.270099 \\
\hline
\end{tabular}




$\begin{array}{lrrr}\mathrm{Ag} & -4.773497 & -0.000189 & 1.727837 \\ \mathrm{Ag} & -2.306294 & -0.000010 & 0.318621 \\ \mathrm{Ag} & -6.229885 & -2.395328 & 1.461481 \\ \mathrm{Ag} & -6.229792 & 2.395209 & 1.461878 \\ \mathrm{Ag} & -3.773849 & 2.360436 & 0.215948 \\ \mathrm{Ag} & -5.222795 & 4.687667 & 0.062664 \\ \mathrm{Ag} & -1.455238 & 3.800734 & -0.649555 \\ \mathrm{Ag} & -2.912796 & 4.711255 & 1.691917 \\ \mathrm{Ag} & -0.000017 & 6.196227 & -0.818934 \\ \mathrm{Ag} & -2.912637 & 6.106355 & -0.774520\end{array}$

